ERNEST DRLANDD LAWRENCE BERKELEY NATIINAL LABDRATIRY

Topics in $\mathbf{N}=1$ Supergravity in Four Dimensions and Superstring Effective Field Theories Beyond Tree-Level

Kamran Saririan

Physics Division
RECEIVED JUL 221997

OSTI

May 1997

Ph.D. Thesis

MAETER 


\section{DISCLAIMER}

This document was prepared as an account of work sponsored by the United States Government. While this document is believed to contain correct information, neither the United States Government nor any agency thereof, nor The Regents of the University of California, nor any of their employees, makes any warranty, express or implied, or assumes any legal responsibility for the accuracy, completeness, or usefulness of any information, apparatus, product, or process disclosed, or represents that its use would not infringe privately owned rights. Reference herein to any specific commercial product, process, or service by its trade name, trademark, manufacturer, or otherwise, does not necessarily constitute or imply its endorsement, recommendation, or favoring by the United States Government or any agency thereof, or The Regents of the University of California. The views and opinions of authors expressed herein do not necessarily state or reflect those of the United States Government or any agency thereof, or The Regents of the University of California. is an equal opportunity employer. 
LBNL-40357

UCB-PTH-97/26

May 1997

\title{
TOPICS IN $N=1$ SUPERGRAVITY IN FOUR DIMENSIONS AND SUPERSTRING EFFECTIVE FIELD THEORIES BEYOND TREE-LEVEL ${ }^{1}$
}

\author{
(Ph.D. Dissertation) \\ Kamran Saririan \\ Department of Physics \\ and \\ Theoretical Physics Group \\ Lawrence Berkeley National Laboratory \\ University of California. \\ Berkeley, CA 94720
}

\begin{abstract}
In this thesis, we present some works in the direction of studying quantum effects in locally supersymmetric effective field theories that appear in the low energy limit of suprestring theory. After reviewing the Kähler covariant formulation of supergravity, we show the calculation of the divergent one-loop contribution to the effective boson Lagrangian for supergravity, including the Yang-Mills sector and the helicity-odd operators that arise from integration over fermion fields. The only restriction is on the Yang-Mills kinetic energy normalization function, which is taken diagonal in gauge indices, as in

\footnotetext{
${ }^{1}$ This work was supported by the Director, Office of Energy Research, Office of High Energy and Nuclear Physics, Division of High Energy Physics of the U.S. Department of Energy under Contract DE-AC03-76SF00098, and the National Science Foundation under grant PHY-95-14797.
}

DISTRIBUTION OF THIS DOCUMENT IS UNLIMITED 
models obtained from superstrings. We then present the full result for the divergent oneloop contribution to the effective boson Lagrangian for supergravity coupled to chiral and Yang-Mills supermultiplets. We also consider the specific case of dilaton couplings in effective supergravity Lagrangians from superstrings, for which the one-loop result is considerably simplified. We study gaugino condensation in the presence of an intermediate mass scale in the hidden sector. S-duality is imposed as an approximate symmetry of the effective supergravity theory. Furthermore, we include in the Kähler potential the renormalization of the gauge coupling and the one-loop threshold corrections at the intermediate scale. It is shown that confinement is indeed achieved. Furthermore, a new running behaviour of the dilaton arises which we attribute to $S$-duality. We also discuss the effects of the intermediate scale, and possible phenomenological implications of this model. 


\section{DISCLAMMER}

Portions of this document may be illegible in electronic image products. Images are produced from the best available original document. 



\subsection{Acknowledgements}

My deepest gratitude goes to my advisor, Professor Mary K. Gaillard, for all the inspiration, support, friendship, and help that she has provided throughout my graduate research at Berkeley. I have benefitted immensely from her keen approach and sense of direction in physics; she has patiently guided me through a difficult but intriguing area of physics, namely superstring phenomenology, and has opened my eyes on lengthy and intricate calculations. In one sentence, her deep intellect and warm personality has made being her student a pleasure.

I would also like to thank Professor Bruno Zumino. Not only his courses have been of great value to me in my education, but much of the underlying subject of this thesis are nothing but some of what he has endowed the world of particle theory. Special thanks also go to Professor Korkut Bardakci who also has taught me a lot about physics, has provided support and encouragement, and participated in my qualifying committee.

There are also others who have made my work in the Theory Group more enjoyable. I am indebted to anyone in the group who has taught me something about physics, and that should include everyone. I would like to acknowledge the help of Dr. I. Hinchliffe on computer matters and useful advice that he has often provided us graduate students of the group. Also, all the help of Barbara Gordon, Mary Kihanya, and Luanne Neumann in making our lives simpler are warmly appreciated. I wish to thank the people who have been on the various committees related to my graduate studies from outside the Theory Group, namely, Professors Nicolai Reshetikhin and Marjori Shapiro. Also, the collaborations with Vidyut Jain and Yi-Yen Wu are gratefully acknowledged. Also a big thank you goes to Anne Takizawa and Donna Sakima of the Physics Department for all the administrative help that they have provided throughout the years, during both my undergraduate and graduate work at Berkeley.

I like to thank my fellow graduate students in the Theory Group, in particular Luis Bernardo and Harold Steinacker for sharing their knowledge with me, and their friendship.

Finally, I cannot possibly thank my parents enough in just a few words. Despite my being forced to leave them and my country at the age of fourteen, and being half-way around the world away from them since then, their love, kindness and encouragement have always given me strength in the positive directions that my life has taken. 
Recycled Paper 
To My Parents 



\section{Contents}

0.1 Acknowledgements ................... i

1 Introduction 1

2 (Classical) Background Material 6

2.1 Kähler Covariant Formulation of Supergravity (A Review) . . . . . . . 6

2.2 The Heterotic String and Compactifications . . . . . . . . 17

3 Supergravity Coupled to YM and Matter at One Loop 26

3.1 Introduction . . . . . . . . . . . . . 26

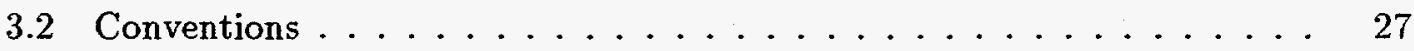

3.3 Dirac algebra ............................. 30

3.4 Quantum Lagrangian and Gauge Fixings (A Summary) . . . . . . . 35

4 The One Loop Calculation $\quad 39$

4.1 Introduction . . . . . . . . . . . . . . . 39

4.2 Gauge Fixing and the Expansion of the Action $\ldots \ldots \ldots$

4.3 Helicity-Odd Fermion Loop Contributions . . . . . . . . . . . . . 49

4.4 The One-Loop Effective Action . . . . . . . . . . . . 60

4.5 The String Dilaton . . . . . . . . . . . . . . 65

4.6 Here Is Where The Action Is. . . . . . . . . . . . 67

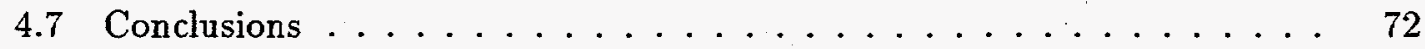

5 Gaugino Condensation, Threshold Corrections, and S-Duality $\quad 74$

5.1 Introduction . . . . . . . . . . . . . . 74

5.2 Gaugino Condensation (A Review) . . . . . . . . . 75

5.3 Duality Symmetries . . . . . . . . . . . . . 76

5.4 The Role of the Gauge Coupling . . . . . . . . . . . . 79

5.5 The Model . . . . . . . . . . . . . . . . . 83

5.6 Scalar Potential and the Vacuum ................ . . 90

5.7 Conclusions . . . . . . . . . . . . . . . 95 



\section{Chapter 1}

\section{Introduction}

Amongst the theories of physics beyond the Standard Model (SM), supersymmetry $[1,2]$, despite the present lack of its present experimental observation, appears to be the strongest candidate. The phenomenological predictions of supersymmetry for physics beyond the SM have been argued to be within experimental reach in the foreseeable future $[2,3]$. If realized in nature, supersymmetry must be broken, and phenomenological consistency requires that it is broken at a scale of order $1 \mathrm{TeV}$ [3].

From the low energy point of view (where by 'low' we mean lower than the scale at which supersymmetry is broken), requiring supersymmetry beyond the electroweak scale has the following theoretical merits. First, supersymmetric theories suffer from perturbative divergences much less than non-supersymmetric theories. This is by the virtue of the cancellations of the boson and fermion contributions to the loop corrections. More specifically, the precise cancellation of the quadratically divergent graphs of fermion loops and boson loops that contribute to the masses of scalars results in the stabilization (i.e., protection against radiative corrections) of the hierarchical mass scales that unfold as one goes across the energy scales (namely, the electroweak scale, $M_{p l}$, and possibly $M_{\mathrm{GUT}}$ which is a mass associated with a grand unified theory much higher than $M_{W}$ ). In other words, supersymmetry provides a partial solution to the gauge hierarchy problem, so that once the origin of the mass scales is explained, supersymmetry makes sure that their relative ratios remain unchanged perturbatively. This merit of supersymmetry is a direct consequence of the nonrenormalization theorems.

The other motivating feature of supersymmetric extension of the SM is that the renormalization group running of the three gauge couplings of $S U(3) \times S U(2) \times U(1)$, $\left(\alpha_{3}, \alpha_{2}, \alpha_{1}\right)$, in the supersymmetric SM unify at about $10^{16} \mathrm{GeV}$ [4]. In contrast, in the nonsupersymmetric minimal SM, the renormalization group equations running of the experimentally measured gauge couplings approach at around $10^{15} \mathrm{GeV}$ but do not meet at the same point, instead there is a notorious mismatch of a few standard deviations. In other words, the MSSM value of $\sin \theta_{W}\left(M_{Z}\right)$ obtained by using the supersymmetric RGEs and assuming unification, agrees remarkably well with the experimental value. 
The standard nonsupersymmetric GUT models were disfavoured because their predictions for proton lifetime is well below the observed lower bound $\sim 10^{32}$ years. Also, GUT without supersymmetry occurs at about $10^{15} \mathrm{GeV}$. One would need to keep the scalars of EW theory massless below this scale. Such a mass is generally generated by loops. It should be mentioned that the unification of the couplings has more theoretical motivation than experimental. However, the experimentally inferred near unification is quite suggestive.

As we go higher energy scales and approach $M_{P l}$, the gravitational effects become stronger. The moment we include gravity, if we insist on having supersymmetry, the physics can nolonger be described by a globally supersymmetric theory. Within the framework of General Relativity, supersymmetry must be gauged, since the algebra of supersymmetry charges contains the momentum vector $p_{\mu}$, i.e., one of the generators (spacetime translations) of the Poincaré group.

This brings us to the motivation for supersymmetry but from the point of view of high energies. Supergravity (locally supersymmetric) theories, as fundamental theories, are nonrenormalizable. However, as effective theories of a more fundamental theory (which presumably contains a consistent description of quantum gravity) they are completely sensible. Currently the only consistent theory of quantum gravity is string theory, which requires supersymmetry for consistency. Furthermore, it provides a unified theory, with the scale of unification of gauge and gravitational couplings at $M_{\text {st }} \sim 2 \times 10^{17}$. This differs from the MSSM value by a factor of $\sim 20$. There are numerous approaches in attempt to account for this apparent discrepancy, and perhaps the most realistic and promising one is the presence of extra massive states in the low energy effective theory of superstrings (a generic feature of most orbifold models) and thus introducing threshold corrections to the running of the gauge couplings.

The recent string duality revolution has taught $u$ s that all the superstring theories that used to be thought of as different theories in fact correspond to different regions of the moduli space of one and the same theory (which in addition contains the 11 dimensional $M$ theory) where a perturbative expansion is possible. In other words, what used to be thought of as different superstring theories, are regions in the moduli space where there exists some definition of weak coupling; although this weak coupling may correspond to strong coupling of another region. These regions, in which the description of physics varies from one to another (say the number of supersymetries, gauge structure, 
or even spacetime dimensions) are mapped into one another by some duality symmetry; for example $\mathrm{S}$ duality (strong-weak duality which in extended supersymmetric gauge theories often translates to a generalization of electric-magnetic duality in electrodynamics with magnetic monopoles) or $\mathrm{T}$ duality (which translates to the duality of a theory compactified on a large space to one on a small space.) The remarkable property that is central to all of the string duality development has been supersymmetry ${ }^{1}$ - without it none of them hold. But that is not the high energy motivation for supersymmetry in four dimensions that we have in mind. If we take superstring theory seriously as a unified fundamental theory which describes the physics beyond $M_{p l}$, and assume that the theory is compactified to four dimensions not too far below that scale, then local supersymmetry in four dimension is forced upon us, because the low energy effective field theories of the superstring in four dimensions are also supersymmetric.

The most phenomenologically successful string theory is the $E_{8} \times E_{8}$ heterotic string theory, whose effective field theory in $4 \mathrm{D}$ is $N=1$ supergravity coupled to super YangMills and matter, with a gauge group that accommodates the SM gauge structure. In addition, the second factor of $E_{8}$ provides a 'hidden' gauge group. The hidden sector can be of great phenomenological importance, namely the strong coupling dynamics of an asymptotically free gauge group of the locally supersymmetric theory in the hidden sector can lead to the breakdown of supersymmetry at a high scale, where the effective theory is still supergravity. The hidden sector can only couple to the observable sector via gravitational-strength interactions or by anomalous $U(1)$ interactions, and through these, a set of soft supersymmetry breaking terms in the observable sector are generated which lead to the breaking of supersymmetry at a scale comparable to the electroweak scale.

In practice, however, making contact between superstring theory and phenomenology has proven extremely difficult. This is mostly due to the vast number of superstring vacua, thereby introducing a huge arbitrariness So on the one hand, superstring theory provides a framework in which there are no arbitrary parameters (all the parameters and couplings are determined by the vevs of the modulus fields - the fields parameterizing the vacuum manifold of the superstring) which is in principle fully predictive. On the other hand, the theory can have a multitude of vacua which limits its predictive power.

\footnotetext{
${ }^{1}$ The duality in four dimensional SYM with $N=2$ supersymmetries [5] and $N=1$ [6] also rely heavily on holomorphy arguments that are direct consequences of supersymmetry.
} 
The vacuum selection, which boils down to lifting the degeneracy of the vacuum states is a problem which gets added to the problem of "how is supersymmetry broken?" in the phenomenological study of superstrings. These problems have long been expected to be one and the same, as it is very suggestive that supersymmetry breaking can lift the flat directions of the theory (or stabilize the moduli fields), and some of the recent developments indicate that this is in fact the case. Closely related to this issue is the notorious 'dilaton runaway' problem, in which, if we assume that the string is weakly coupled, and do not include any nonperturbative effects with a stringy origin, we can expect that the potential that arises for the dilaton (the modulus field whose vev determines the YM gauge coupling in the effective field theory) has its only stable minimum in the extreme weak coupling, supersymmetry preserving limit.

Since the early days of superstring phenomenology, it has been apparent that in order to make contact between the underlying theory and the effective field theory it would be necessary to include quantum corrections in both. An early example is the string threshold effects and the loop corrections to the gauge coupling of the locally supersymmetric effective theory giving the moduli dependence of the latter, and the connection with the modular invariance (exact in string theory) of the effective theory. Modular symmetry is anomalous in the effective supergravity theory. To fully restore this invariance in the effective theory, i.e., to determine the counter-terms which cancel the anomaly, requires the understanding of the divergences in the locally supersymmetric effective field theory. The renormalization of the Kähler potential is also relevant in lifting certain flat directions in a supergravity effective theory. The determination of the full loop corrections may also have nontrivial effects in the study of gaugino condensation as a mechanism for supersymmetry breaking.

In this thesis, we shall describe many of the above issues in supergravity effective theories in detail. In particular, we shall describe some works which are in the direction of including quantum effects in the locally supersymmetric effective field theories which include YM gauge fields as well as matter, with field dependent gauge couplings, i.e., superstring inspired supergravity. We shall discuss the calculation of the divergent one loop corrections to the above system. We shall also discuss non-perturbative corrections in the context of a specific model of gaugino condensation in the hidden sector, which also includes perturbative corrections to the Kähler potential as well as non-perturbative effect, which is $\mathrm{S}$ duality imposed as an approximate symmetry. 
The organization of the thesis is as follows. In Chapter 2 we give a rather brief review of the Kähler covariant formulation of supergravity which is the framework used here essentially throughout. In that chapter we also recall some basic facts of life about superstrings and the effective quantum field theories which they give rise to upon compactifications, with heavier emphasis on the heterotic strings which are the most relevant for our applications. Chapter 3 essentially sets the notation and outlines the strategy used in calculation of the one loop effective Lagrangian of the supergravity, YM, matter system. This calculation is discussed thoroughly in Chapter 4 . In Chapter 5 we first present a brief discussion of gaugino condensation in the hidden sector, and also of duality symmetries (modular invariance and $\mathrm{S}$ duality). We then examine a superstring inspired toy model of gaugino condensation which incorporates certain radiative corrections to the Kähler potential, as well as $\mathrm{S}$ duality and modular invariance as underlying approximate and exact symmetries, respectively. 


\section{Chapter 2}

\section{(Classical) Background Material}

\subsection{Kähler Covariant Formulation of Supergravity (A Review)}

As was first pointed out by Zumino [7], supersymmetric $\sigma$-model has Kähler symmetry, i.e., the complex scalars of the matter supermultiplets parameterize a Kähler manifold [10]. This Kähler symmetry is an invariance of the mater coupled to supergravity system, provided that the fermions undergo a chiral (and field dependent) phase transformation [11].

In the Kähler covariant formalism [13] Kähler transformations have a geometrical interpretation at the superfield ${ }^{1}$ level, as they appear in the structure group on equal footing with Lorentz transformations.

In the following, a brief description of supergravity in the Kähler covariant formulation is given, following mainly reference [12]. After constructing the superspace action, eventually, we outline the essential steps in obtaining the component tree level Lagrangian for supergravity, super Yang-Mills (YM) and matter system.

The 2-component spinor notations used here in this chapter are those of ref. [12] which are more or less standard in supersymmetry [10]. We do not list them here because the intention of this chapter is not to discuss the details of the formalism, but to give an overview of the structure of the Kähler superspace and to sketch out how one obtains the component form of the Lagrangian. In Chapter 3, where we discuss the 1-loop effective Lagrangian (which also uses slightly different notation than here), we shall be much more explicit about our notation.

First, Kähler transformations $K(\varphi, \bar{\varphi}) \rightarrow K(\varphi, \bar{\varphi})+F(\varphi)+\bar{F}(\bar{\varphi})$ (where $\varphi$ is any chiral superfield) is accompanied by the transformation

$$
\Omega \rightarrow \Omega e^{\frac{-i}{2} w \operatorname{Im} F}
$$

where $w$ is the chiral $\mathrm{U}(1)$ weight of the superfield $\Omega$ under Kähler transformations.

\footnotetext{
${ }^{1}$ We assume that the reader is familiar with the basics of superspace and supersymmetry, at the level of the introductory chapters of the text by Wess and Bagger [10], as well as with General Relativity and differential geometry $[8,9]$.
} 
Furthermore, the superspace covariant derivative is also covariant with respect to Kähler transformation, as we shall see. Let us introduce the superspace coordinates $z^{M} \sim$ $\left(x^{m}, \theta^{\mu}, \bar{\theta}_{\dot{\mu}}\right)$, and the local frame coordinates $\left(x^{a}, \theta^{\alpha}, \bar{\theta}_{\dot{\alpha}}\right)$ such that

$$
D_{A}=E_{A}^{M} \partial_{M}=\left(\frac{\partial}{\partial x^{a}}, D_{\alpha}, D^{\dot{\alpha}}\right)
$$

where $M=(m, \mu, \dot{\mu}), A=(a, \alpha, \dot{\alpha})$, and $D_{\alpha}$ and $D_{\dot{\alpha}}$ are the ordinary spinorial derivative operators, for example,

$$
D_{\alpha}=\frac{\partial}{\partial \theta^{\alpha}}+i \bar{\theta}_{\dot{\alpha}}\left(\bar{\sigma}^{m} \epsilon\right)_{\alpha}^{\dot{\alpha}} \frac{\partial}{\partial x^{m}} .
$$

Here $\bar{\sigma}^{0}=\sigma^{0}$ and $\bar{\sigma}^{1,2,3}=-\sigma^{1,2,3}$ The derivatives $D_{A}$ satisfy $D_{C} D_{B}-(-1)^{b c} D_{B} D_{C}=$ $-T_{C B}{ }^{A} D_{A}$ with $b=0,1$ for vectorial and spinorial index $B$, respectively (likewise for c); $T_{C B}{ }^{A}$ are the components of the torsion in superspace. In equation (2.2), $E_{A}{ }^{M}$ is the inverse super-vielbein which in the flat spacetime limit reduces to:

$$
E_{A}{ }^{M}=\left(\begin{array}{ccc}
\delta_{a}^{m} & 0 & 0 \\
i\left(\bar{\theta} \bar{\sigma}^{m} \epsilon\right)_{\alpha} & \delta_{\alpha}^{\mu} & 0 \\
i\left(\theta \sigma^{m} \epsilon\right)^{\dot{\alpha}} & 0 & \delta_{\dot{\mu}}^{\dot{\alpha}}
\end{array}\right) .
$$

The torsion two-form is then given by:

$$
T^{A}=d E^{A}+E^{B} \phi_{B}^{A}+w\left(E^{A}\right) E^{A} A
$$

where $E^{A}=d z^{M} E_{M}{ }^{A}$ and $\phi_{B}{ }^{A}=d z^{M} \phi_{M B}{ }^{A}(z)$ is the Lorentz gauge connection. The last term in (2.4) corresponds to covariantization with respect to Kähler transformations: $A=d z^{M} A_{M}$ is the connection one-form for the Kähler transformations. The Kähler weights $w\left(E^{A}\right)$ is given by $w\left(E^{a}\right)=0, w\left(E^{\alpha}\right)=1$, and $w\left(E_{\dot{\alpha}}\right)=-1$. The superspace one-form $A$ is given by (see [13])

$$
\begin{aligned}
A_{\alpha} & =\frac{1}{4} E_{\alpha}{ }^{M} \partial_{M} K(\varphi, \bar{\varphi}) \\
A^{\dot{\alpha}} & =-\frac{1}{4} E^{\dot{\alpha} M} \partial_{M} K(\varphi, \bar{\varphi}) \\
A_{\alpha \dot{\alpha}}-\frac{3 i}{2} G_{\alpha \dot{\alpha}} & =\frac{i}{2}\left(\mathcal{D}_{\alpha} A_{\dot{\alpha}}+\mathcal{D}_{\dot{\alpha}} A_{\alpha}\right)=-\frac{i}{8}\left[\mathcal{D}_{\alpha}, \mathcal{D}_{\dot{\alpha}}\right] K
\end{aligned}
$$

where $G_{\alpha \dot{\alpha}}=\sigma_{\alpha \dot{\alpha}}^{a} G_{a}$ is an auxiliary field which is related to the spinorial component of the $U_{K}(1)$ field strength (namely, $F_{\beta}^{\dot{\alpha}}=3\left(\sigma^{a} \epsilon\right)_{\beta}^{\dot{\alpha}} G_{a}$ ). The other objects of interest are the superfields $X_{\alpha}$ and $\bar{X}^{\dot{\alpha}}$ given by:

$$
\begin{aligned}
X_{\alpha} & =-\frac{1}{8}\left(\mathcal{D}_{\dot{\alpha}} \mathcal{D}^{\dot{\alpha}}-8 R\right) \mathcal{D}_{\alpha} K \\
\bar{X}^{\dot{\alpha}} & =-\frac{1}{8}\left(\mathcal{D}_{\alpha} \mathcal{D}^{\alpha}-8 R\right) \mathcal{D}^{\dot{\alpha}} K .
\end{aligned}
$$


The chiral superfield $R$ will be discussed momentarily, but let us first reexpress the $X$ fields as (see [12]):

$$
\begin{aligned}
& X_{\alpha}=\frac{-i}{2} K_{k \bar{k}} \sigma_{\alpha \dot{\alpha}}^{a} \mathcal{D}_{a} \varphi^{k} \mathcal{D}^{\dot{\alpha}} \bar{\varphi}^{\bar{k}}+\frac{1}{2} K_{k \bar{k}} \mathcal{D}_{\alpha} \varphi^{k} \bar{F}^{\bar{k}} \\
& \bar{X}^{\dot{\alpha}}=\frac{-i}{2} K_{k \bar{k}} \bar{\sigma}^{a \alpha \dot{\alpha}} \mathcal{D}_{a} \bar{\varphi}^{\bar{k}} \mathcal{D}_{\alpha} \varphi^{k}+\frac{1}{2} K_{k \bar{k}} \mathcal{D}^{\dot{\alpha}} \bar{\varphi}^{\bar{k}} F^{k}
\end{aligned}
$$

It follows from the definitions of $X_{\alpha}$ and $\bar{X}^{\dot{\alpha}}$ that they satisfy:

$$
\mathcal{D}^{\dot{\alpha}} X_{\alpha}=0, \quad \mathcal{D}_{\alpha} \bar{X}^{\dot{\alpha}}=0, \quad \mathcal{D}^{\alpha} X_{\alpha}=\mathcal{D}_{\dot{\alpha}} \bar{X}^{\dot{\alpha}}
$$

In the component form of the action, we shall find that the lowest components of the superfields $X_{\alpha}$ and $\bar{X}^{\dot{\alpha}}$ will appear. Also relevant to the construction of the component field action are the chiral superfields $R$ and $R^{\dagger}$ (with $w(R)=-w\left(R^{\dagger}\right)=2$ ), whose $F$-components involve the Riemann tensor, and satisfy:

$$
\begin{aligned}
\mathcal{D}_{\alpha} R & =-\frac{1}{3} X_{\alpha}-\frac{2}{3}\left(\sigma^{c b} \epsilon\right)_{\alpha \varphi} T_{\varphi c b} \\
\mathcal{D}^{\dot{\alpha}} R^{\dagger} & =-\frac{1}{3} \bar{X}^{\dot{\alpha}}-\frac{2}{3}\left(\bar{\sigma}^{c b} \epsilon\right)^{\dot{\alpha} \dot{\varphi}} T_{c b \dot{\varphi}}
\end{aligned}
$$

where $T_{c b}^{\varphi}$ and $T_{c b \dot{\varphi}}$ are the components of the torsion superfield, and $\left(\sigma^{c b}\right)_{\alpha}{ }^{\beta} \equiv \frac{1}{4}\left(\sigma^{c} \bar{\sigma}^{b}-\right.$ $\left.\sigma^{b} \bar{\sigma}^{c}\right)_{\alpha}^{\beta}$ Applying the Bianchi identity along with taking another spinorial derivative in (2.10) imply that:

$$
\mathcal{D}^{\alpha} \mathcal{D}_{\alpha} R+\mathcal{D}_{\dot{\alpha}} \mathcal{D}^{\dot{\alpha}} R^{\dagger}=-\frac{2}{3} R_{a b}^{a b}-\frac{1}{3}\left(\mathcal{D}^{\alpha} X_{\alpha}+\mathcal{D}_{\dot{\alpha}} \bar{X}^{\dot{\alpha}}\right)-4 G_{a} G^{a}+32 R R^{\dagger}
$$

\section{Introducing Yang-Mills Fields}

The YM connection is

$$
\mathcal{A}=E^{A} \mathcal{A}_{A}^{r} T_{r}=\mathcal{A}^{r} T_{r}
$$

where the indices $r$ are the Lie algebra indices. The YM field strength two-form

$$
\mathcal{F}=\frac{1}{2} E^{A} E^{B} \mathcal{F}_{B A}=\mathcal{F}^{r} T_{r}
$$

is given by the usual expression in terms of the YM connection:

$$
\mathcal{F}^{r}=d \mathcal{A}^{r}+\frac{i}{2} \mathcal{A}^{p} \mathcal{A}^{q} c_{p q}^{r}
$$

where the coefficients $c_{p q}^{r}$ are the structure constants of the Lie algebra. Spinorial components of $\mathcal{F}$ satisfy the covariant constraints:

$$
\mathcal{F}^{\dot{\alpha} \dot{\beta}}=0, \quad \mathcal{F}_{\alpha \beta}=0, \quad \mathcal{F}_{\beta}^{\dot{\alpha}}=0
$$


which together with the Bianchi identities constrain the other components of $\mathcal{F}$ as follows:

$$
\begin{aligned}
\mathcal{F}_{\beta a} & =i\left(\sigma_{a} \epsilon\right)_{\beta}^{\dot{\alpha}} \mathcal{W}_{\dot{\alpha}} \\
\mathcal{F}^{\dot{\beta} a} & =i\left(\bar{\sigma}^{a} \epsilon\right)_{\alpha}^{\dot{\beta}} \mathcal{W}^{\alpha} \\
\mathcal{F}_{a b} & =\frac{1}{2}\left(\epsilon \sigma_{a b}\right)^{\alpha \beta} \mathcal{D}_{\beta} \mathcal{W}_{\alpha}+\frac{1}{2}\left(\bar{\sigma}_{a b} \epsilon\right)^{\dot{\alpha} \dot{\beta}} \mathcal{D}_{\dot{\beta}} \mathcal{W}_{\dot{\alpha}}
\end{aligned}
$$

where $\mathcal{W}_{\alpha}=\mathcal{W}_{\alpha}^{r} T_{r}$ and $\mathcal{W}^{\dot{\alpha}}=\mathcal{W}^{r \dot{\alpha}} T_{r}$ are the Yang-Mills superfields of Kähler weight +1 and -1 , respectively. They satisfy, as a result of the Bianchi identities and the constraints, the well known equations:

$$
\mathcal{D}_{\alpha} \mathcal{W}^{\dot{\alpha}}=0, \quad \mathcal{D}^{\dot{\alpha}} \mathcal{W}_{\alpha}=0, \quad \mathcal{D}^{\alpha} \mathcal{W}_{\alpha}=\mathcal{D}_{\dot{\alpha}} \mathcal{W}^{\dot{\alpha}}
$$

It should be noted, especially as we have not been terribly explicit, that solving the YM Bianchi identities makes use of the complete structure of the $U_{K}(1) \mathrm{Kähler}$ superspace, i.e., the derivatives are covariant with respect to $\mathrm{YM}$ as well as Lorentz and $U_{K}(1)$ gauge transformations [12]. In the presence of YM fields, the previous discussion is modified, and the appropriate modification of eq. (2.7) and (2.8) leads to

$$
\begin{aligned}
X_{\alpha} & =\frac{-i}{2} K_{k \bar{k}} \sigma_{\alpha \dot{\alpha}}^{a} \mathcal{D}_{a} \varphi^{k} \mathcal{D}^{\dot{\alpha}} \bar{\varphi}^{\bar{k}}+\frac{1}{2} K_{k \bar{k}} \mathcal{D}_{\alpha} \varphi^{k} \bar{F}^{\bar{k}}+\mathcal{W}_{\alpha}^{\tau} D_{r} \\
\bar{X}^{\dot{\alpha}} & =\frac{-i}{2} K_{k \bar{k}} \bar{\sigma}^{a \alpha \dot{\alpha}} \mathcal{D}_{a} \bar{\varphi}^{\bar{k}} \mathcal{D}_{\alpha} \varphi^{k}+\frac{1}{2} K_{k \bar{k}} \mathcal{D}^{\dot{\alpha}} \bar{\varphi}^{\bar{k}} F^{k}+\mathcal{W}^{r \dot{\alpha}} D_{r}
\end{aligned}
$$

The Kähler D-term that appears in the r.h.s. of eq. (2.11) is:

$$
\begin{aligned}
-\frac{1}{2} \mathcal{D}^{\alpha} X_{\alpha}= & -K_{k \bar{\jmath}} \mathcal{D}_{a} \varphi^{k} \mathcal{D}^{\alpha} \bar{\varphi}^{\bar{\jmath}}-\frac{i}{4} K_{k \bar{\jmath}} \sigma_{\alpha \dot{\alpha}}^{a}\left(\mathcal{D}^{\alpha} \varphi^{k} \mathcal{D}_{a} \mathcal{D}^{\dot{\alpha}} \bar{\varphi}^{\bar{\jmath}}+\mathcal{D}^{\dot{\alpha}} \bar{\varphi}^{\bar{\jmath}} \mathcal{D}_{a} \mathcal{D}^{\alpha} \varphi^{k}\right) \\
& +K_{k \bar{k}} F^{k} \bar{F}^{\bar{k}}+\frac{1}{16} R_{j \bar{\jmath} k \bar{k}} \mathcal{D}^{\alpha} \varphi^{k} D_{\alpha} \varphi^{j} \mathcal{D}_{\dot{\alpha}} \bar{\varphi}^{\bar{k}} \mathcal{D}^{\dot{\alpha}} \bar{\varphi}^{\bar{\jmath}} \\
& -i K_{k \bar{k}} \bar{V}_{r}^{\bar{k}} \mathcal{W}_{\alpha}^{r} \mathcal{D}^{\alpha} \varphi^{k}-i K_{k \bar{k}} V_{r}^{k} \mathcal{W}_{\dot{\alpha}}^{r} \mathcal{D}^{\dot{\alpha}} \bar{\varphi}^{\bar{k}}-\frac{1}{2}\left(\mathcal{D}^{\alpha} \mathcal{W}_{\alpha}^{r}\right) D_{r},
\end{aligned}
$$

where

$$
R_{i \bar{z} j \bar{j}}=\partial_{i} \partial_{\bar{\imath}} K_{j \bar{j}}-K^{k \bar{k}} K_{i \bar{k}, j} K_{k \bar{i}, \bar{j}}
$$

where, $V_{r}^{k}$ and $\bar{V}_{r}^{\bar{k}}$ are the holomorphic (antiholomorphic) generators of the gauge symmetry defined by variations of the coordinates of the Kähler manifold,

$$
\begin{aligned}
\delta_{\alpha} \varphi^{k} & =-\alpha^{r} V_{r} \varphi^{k}, & \delta_{\alpha} \bar{\varphi}^{\bar{k}}=-\alpha^{r} \bar{V}_{r} \bar{\varphi}^{\bar{k}} \\
V_{r} & =V_{r}^{k}(\varphi) \frac{\partial}{\partial \varphi^{k}}, & \bar{V}_{r}=\bar{V}_{r}^{k}(\bar{\varphi}) \frac{\partial}{\partial \bar{\varphi}^{\bar{k}}} \\
V_{r}^{k} & =-i\left(T_{r} \varphi\right)^{k}, & \bar{V}_{r}^{\bar{k}}=-i\left(T_{r} \bar{\varphi}\right)^{\bar{k}}
\end{aligned}
$$


the generators satisfy the underlying Lie algebra commutation relations $\left[V_{r}, V_{s}\right]=c_{r s}{ }^{t} V_{t}$ (likewise for $\bar{V}$ 's, and $\left[V_{r}, \bar{V}_{s}\right]=0$ ). Furthermore, the Killing potentials (i.e., solutions of the Killing equations for the metric) $D_{r}(\varphi, \bar{\varphi})$, are given locally in terms of the generators and the Kähler metric $K_{i \bar{\jmath}}=\frac{\partial^{2} K(\varphi, \bar{\varphi})}{\partial \varphi^{k} \partial \bar{\varphi}^{k}}$ in :

$$
K_{i \bar{\jmath}} \bar{V}_{\tau}^{\bar{\jmath}}=i \frac{\partial D(\varphi, \bar{\varphi})}{\partial \varphi^{i}}, \quad K_{i \bar{\jmath}} V_{\tau}^{i}=-i \frac{\partial D(\varphi, \bar{\varphi})}{\partial \bar{\varphi}^{\hat{\jmath}}}
$$

The Killing potentials $D_{r}$ are in turn solved for in terms of the holomorphic and antiholomorphic functions $F_{r}$ and $\bar{F}_{r}$ which arise as the Kähler transformation induced by the action of the gauge symmetry generators on the Kähler potential: $\left(V_{r}+\bar{V}_{r}\right) K=F_{r}+\bar{F}_{r}$. We simply quote the result that the solutions to the equations $(2.22)$ is: $D_{r}=K_{i}\left(T_{r} \varphi\right)^{i}$.

\section{LOCAL SUPERSYMMETRY TRANSFORMATIONS}

In the following we shall outline the construction of the supersymmetric Lagrangian for supergravity coupled to Yang-Mills and (chiral) matter fields with the above ingredients. The matter fields parameterize a Kähler manifold. The transformations of the objects $E^{A}$ (the superspace frame, or super-vielbein), matter superfields, $\varphi^{k}$ and $\bar{\varphi}^{\bar{k}}$ and the YM connection one-form $\mathcal{A}^{r}=d z^{M} \mathcal{A}_{M}^{r}$ under the superspace diffeomorphisms, Lorentz and YM gauge transformations $\left(\xi_{M}, \Lambda_{B}{ }^{A}\right.$, and $\alpha^{r}$, respectively) are:

$$
\begin{aligned}
\delta E^{A} & =L_{\xi} E^{A}+E^{B} \Lambda_{B}{ }^{A}-\frac{i}{2} w\left(E^{A}\right) E^{A} \operatorname{Im}\left(F(\varphi)-\alpha^{r} F_{r}(\varphi)\right) \\
\delta \varphi^{k} & =L_{\xi} \varphi^{k}-\alpha^{r} V_{r}^{k}(\varphi) \\
\delta \bar{\varphi}^{\bar{k}} & =L_{\xi} \bar{\varphi}^{\bar{k}}-\alpha^{r} \bar{V}_{r}^{\bar{k}}(\bar{\varphi}) \\
\delta \mathcal{A}^{r} & =L_{\xi} \mathcal{A}^{r}-i d \alpha^{r}+\alpha^{p} \mathcal{A}^{q} c_{p q}{ }^{r}
\end{aligned}
$$

where $L_{\xi}=i_{\xi} d+d i_{\xi}$ is the Lie derivative in superspace and $i_{\xi}$ and $d$ are respectively the interior product with respect to the superspace vector $\xi^{A}$ and the exterior derivative. The compensating $\xi$-dependent Lorentz and YM transformations correspond to $\Lambda_{B}{ }^{A}=i_{\xi} \phi_{B}{ }^{A}=i_{\xi} d x^{C} \phi_{C B}{ }^{A}$ and $\alpha^{r}=-i_{\xi} \mathcal{A}^{r}[12,10]$. The above local supersymmetry transformations can then be cast into the following form which will prove more convenient later when we derive the transformations of the component fields:

$$
\begin{aligned}
\delta E_{M}{ }^{A} & =\mathcal{D}_{M} \xi^{A}+E_{M}{ }^{B} \xi^{C} T_{C B}{ }^{A}-\frac{1}{4} w\left(E^{A}\right) E_{M}{ }^{A} \xi^{B}\left(K_{k} \mathcal{D}_{B} \varphi^{k}-K_{\bar{k}} \mathcal{D}_{B} \dot{\varphi}^{\bar{k}}\right) \\
& -\frac{i}{8} w(E) E_{M}{ }^{A} \xi^{b}\left(12 G_{b}+\bar{\sigma}_{b}^{\dot{\alpha} \alpha} K_{k \bar{k}} \mathcal{D}_{\alpha} \varphi^{k} \mathcal{D}_{\dot{\alpha}} \bar{\varphi}^{\bar{k}}\right)
\end{aligned}
$$




$$
\begin{aligned}
\delta \varphi^{k} & =\xi^{A} \mathcal{D}_{A} \varphi^{k}, \delta \bar{\varphi}^{\bar{k}}=\xi^{A} \mathcal{D}_{A} \bar{\varphi}^{\bar{k}} \\
\delta \Gamma & =x i^{A} \mathcal{D}_{A} \Gamma-\frac{1}{4} w(\Gamma) \Gamma \xi^{A}\left(K_{k} \mathcal{D}_{A} \varphi^{k}-K_{\bar{k}} \mathcal{D}_{A} \bar{\varphi}^{\bar{k}}\right) \\
& -\frac{i}{8} w(\Gamma) \Gamma \xi^{b}\left(12 G_{b}+\bar{\sigma}_{b}^{\dot{\alpha} \alpha} K_{k \bar{k}} \mathcal{D}_{\alpha} \varphi^{k} \mathcal{D}_{\dot{\alpha}} \bar{\varphi}^{\bar{k}}\right) \\
\delta u^{k} & =\xi^{A} \mathcal{D}_{A} u^{k}+\Gamma_{\ell m}^{k} \xi^{A} \mathcal{D}_{A} \varphi^{\ell} u^{m},
\end{aligned}
$$

where $\Gamma$ and $u^{k}$ are generic superfields which are assumed to have the following transformation laws:

$$
\begin{aligned}
\delta u^{k} & =L_{\xi} u^{k}-\alpha^{r} \frac{\partial V_{r}^{k}(\varphi)}{\partial \varphi^{\ell}} u^{\ell} \\
\delta \Gamma & =L_{\xi} d \Gamma-\frac{i}{2} w(\Gamma) \Gamma \operatorname{Im}\left(F(\varphi)-\alpha^{\tau} F_{r}(\varphi)\right)
\end{aligned}
$$

The reason for introducing these generic fields will become clear later in our discussion of the component field supersymmetry transformation.

\section{INVARIANT SUPERFIELD ACTIONS}

Invariant actions are obtained by integrating superspace densities over all directions (commuting and anticommuting) of the superspace. Remarkably, the supergravity plus matter action is simply the integral over the volume element of the superspace constructed above:

$$
\mathcal{L}_{\text {sugra+matt }}=-3 \int E \equiv \mathcal{L}_{\text {sugra }}+e \mathcal{D}_{\text {matter }},
$$

where the integration over $d^{4} x d^{4} \theta$ is understood. $E$ is the super-determinant of the superspace vielbein $E_{M}{ }^{A}, e=\operatorname{det} e_{m}{ }^{a}$, and $\mathcal{D}_{\text {matter }}$ is the terms induced by Kähler superspace structure and contains the matter field contributions:

$$
\mathcal{D}_{\text {matter }}=-\frac{1}{2} \mathcal{D}^{\alpha} X_{\alpha}\left|+\frac{i}{2} \psi_{m}^{\alpha} \sigma_{\alpha \dot{\alpha}}^{m} \bar{X}^{\dot{\alpha}}\right|+\frac{i}{2} \bar{\psi}_{m \alpha} \bar{\sigma}_{m \alpha \dot{\alpha}} X_{\alpha} \mid
$$

where the vertical bars denote the projection to the lowest component of the superfield. We shall discuss the matter contributions to some detail later on. The Yang-Mills and superpotential contributions are:

$$
\mathcal{L}_{\mathrm{YM}}=\int \frac{E}{8 R} f_{r s}(\varphi) \mathcal{W}^{r \alpha} \mathcal{W}_{\alpha}^{s}+\int \frac{E}{8 R^{\dagger}} \bar{f}_{r s}(\bar{\varphi}) \mathcal{W}_{\dot{\alpha}}^{r} \mathcal{W}^{s \dot{\alpha}}
$$

and

$$
\mathcal{L}_{\mathrm{sp}}=\frac{1}{2} \int \frac{E}{R} e^{K} W(\varphi)+\frac{1}{2} \int \frac{E}{R^{\dagger}} e^{K} \bar{W}(\bar{\varphi})
$$


Where the gauge kinetic function, $f_{r s}$ and the superpotential $W$ are holomorphic functions of the chiral fields. The Kähler invariance is verified by recalling that the Kähler weights of $R$ and $R^{\dagger}$ are +2 and -2 , respectively, and since $w\left(\mathcal{W}_{\alpha}\right)=-w\left(\mathcal{W}_{\dot{\alpha}}\right)=1$, for the YM action to be $U_{K}(1)$ invariant, the gauge kinetic function, $f_{r s}$ must have zero Kähler weight. Furthermore, $w\left(e^{K / 2} W\right)=2=-w\left(e^{K / 2} \bar{W}\right)$, hence $\mathcal{L}_{\text {sp }}$ is Kähler invariant.

As for gauge invariance, the YM term is invariant provided that the gauge kinetic functions satisfy

$$
V_{p} f_{r s}=c_{p r}{ }^{q} f_{q s}+c_{p s}{ }^{q} f_{q r}
$$

and the superpotential term is invariant if

$$
\delta W=\alpha^{r} F_{r}(\varphi) W(\varphi)
$$

Finally, since the action is directly written in superspace, supersymmetry is automatic by construction.

Do the Lagrangians in (2.26)-(2.29) correctly describe the dynamics of supergravity, matter, and Yang-Mills system? The justifications are the superfield equations of motion, and also the component-field actions corresponding to the superfield actions (2.26)-(2.29). For details of the former approach (i.e., the derivation of the equations of motion) we refer the reader to ref. [12]. Here we shall discuss the component fields, mainly because the starting point of our calculation of the 1-loop effective Lagrangian of supergravity, YM and matter system is the component-field tree-level Lagrangian for the system, which we shall construct next.

\section{COMPONENT Fields}

The supergravity multiplet consists of the vielbein $e_{m}{ }^{a}$, the gravitino, $\left(\psi_{m}^{\alpha}, \bar{\psi}_{m \dot{\alpha}}\right)$, and two auxiliary fields, $M$ (complex scalar) and vector field $b_{a}$. These are given by the $\theta=\bar{\theta}=0$ components of the supervielbein, $E_{M}{ }^{A}$ (in the Wess-Zumino gauge (see [10], for example)) and of $R$ and $G_{a}$ superfields:

$$
\begin{gathered}
E_{M}{ }^{A} \mid=\left(\begin{array}{ccc}
e_{m}{ }^{a} & \frac{1}{2} \psi_{m}^{\alpha} & \frac{1}{2} \bar{\psi}_{m \dot{\alpha}} \\
0 & \delta_{\alpha}^{\mu} & 0 \\
0 & 0 & \delta_{\dot{\alpha}}^{\dot{\mu}}
\end{array}\right), \\
R\left|=-\frac{1}{6} M, \quad R^{\dagger}\right|=-\frac{1}{6} \bar{M}, \quad G_{a} \mid=-\frac{1}{3} b_{a},
\end{gathered}
$$


where the vertical bar indicates projection to the lowest component $(\theta=\bar{\theta}=0)$ of the superfield. For the components $\omega_{m B}{ }^{A}$ of the connection $\left(\phi_{B}{ }^{A}=d x^{m} \omega_{m B}{ }^{A}\right)$ and torsion $T_{M N}{ }^{A}$ and $T_{C B}{ }^{A}$ which are related by

$$
T_{M N}^{A}=(-1)^{n(m+b)} E_{M}^{B} E_{N}^{C} T_{C B}{ }^{A}
$$

The lowest component projection of the superfields $X_{\alpha}$ and $\bar{X}^{\dot{\alpha}}$ of eq. (2.18) are thus obtained using: ${ }^{2}$

$$
\begin{aligned}
\varphi^{i} \mid & =A^{i}, \quad \mathcal{D}_{\alpha} \varphi^{i}\left|=\sqrt{2} \chi_{\alpha}^{i}, \quad \mathcal{D}^{\alpha} \mathcal{D}_{\alpha} \varphi^{i}\right|=-4 F^{i} \\
\mathcal{A}_{m} \mid & =i a_{m}, \quad \mathcal{W}^{\dot{\beta}}\left|=i \lambda^{\dot{\beta}}, \quad \mathcal{D}^{\alpha} \mathcal{W}_{\alpha}\right|=-2 \mathbf{D} \\
\mathcal{D}_{a} \varphi \mid & =E_{a}{ }^{M} \mathcal{D}_{M} \varphi^{i}\left|=\left(e_{a}{ }^{m} \mathcal{D}_{m} \varphi^{i}-\frac{1}{2} e_{a}{ }^{m} \psi_{m}^{\alpha} \delta_{\alpha}^{\mu} \mathcal{D}_{\mu} \varphi^{i}\right)\right| \\
& =e_{a}{ }^{m}\left(\mathcal{D}_{m} A^{i}-\frac{1}{\sqrt{2}} \psi_{m}^{\alpha} \chi_{\alpha}^{i}\right) .
\end{aligned}
$$

Then it is straightforward to show that

$$
X_{\alpha}=-\frac{i}{\sqrt{2}} K_{k \bar{k}} \sigma_{\alpha \dot{\alpha}}^{m} \bar{\chi}^{\bar{k} \dot{\alpha}}\left(\mathcal{D}_{m} A^{k}-\frac{1}{\sqrt{2}} \psi_{m}^{\beta} \chi_{\beta}^{k}\right)+\frac{1}{\sqrt{2}} K_{k \bar{k}} \chi_{\alpha}^{k} \bar{F}^{k}-i \lambda_{\alpha}^{r} D_{r}
$$

Now let us consider the superfield $-\frac{1}{2} \mathcal{D}^{\alpha} X_{\alpha}$, as given in eq. (2.19). Using:

$$
\mathcal{D}_{a} \mathcal{D}^{\alpha} \varphi^{i} \mid=e_{a}^{m}\left[\sqrt{2} \mathcal{D}_{m} \chi^{\alpha i}-\psi_{m}^{\alpha} F^{i}+i\left(\bar{\psi}_{m} \bar{\sigma}^{n}\right)^{\alpha}\left(\mathcal{D}_{n} A^{i}-\frac{1}{\sqrt{2}} \psi_{n}^{\beta} \chi_{\beta}^{i}\right)\right]
$$

one obtains:

$$
\begin{aligned}
-\frac{1}{2} \mathcal{D}^{\alpha} X_{\alpha} & =-K_{i \bar{\jmath}} g^{m n} \mathcal{D}_{m} A^{i} \mathcal{D}_{n} \bar{A}^{\bar{\jmath}}-\frac{i}{2} K_{i \bar{\jmath}} \chi^{\alpha i} \sigma_{\alpha \dot{\alpha}}^{m} \mathcal{D}_{m} \bar{\chi}^{\bar{\jmath} \dot{\alpha}} \\
& +\frac{i}{2}\left(\mathcal{D}_{m} \chi^{\alpha i}\right) \sigma_{\alpha \dot{\alpha}}^{m} K_{i \bar{\jmath}} \bar{\chi}^{\dot{\alpha} \bar{\jmath}}+K_{i \bar{\jmath}} F^{i} \bar{F}^{\bar{\jmath}}+\frac{1}{2} K_{i \bar{\jmath}} g^{m n}\left(\psi_{m} \chi^{i}\right)\left(\bar{\psi}_{n} \bar{\chi}^{\bar{\jmath}}\right) \\
& +\frac{1}{4} R_{i \bar{\jmath} j \bar{\jmath}}\left(\chi^{i} \chi^{j}\right)\left(\bar{\chi}^{\bar{\imath}} \bar{\chi}^{\bar{\jmath}}\right)+\sqrt{2}\left(\chi^{i} \lambda^{r}\right) K_{i \bar{\jmath}} \bar{V}_{r}^{\bar{\jmath}}+\sqrt{2}\left(\bar{\chi}^{\bar{\jmath}} \bar{\lambda}^{r}\right) K_{i \bar{\jmath}} V_{r}^{i} \\
& +\mathbf{D}^{r} D_{r}-\frac{i}{2 \sqrt{2}}\left(\bar{\psi}_{m} \bar{\sigma}^{m} \chi^{i}\right) K_{i \bar{\jmath}} \bar{F}^{\bar{j}}-\frac{i}{2 \sqrt{2}}\left(\psi_{m} \sigma^{m} \bar{\chi}^{\bar{\jmath}}\right) K_{i \bar{\jmath}} F^{i} \\
& -\frac{1}{2 \sqrt{2}}\left(\bar{\psi}_{m} \bar{\sigma}^{n} \sigma^{m} \bar{\chi}^{\bar{\jmath}}-\bar{\psi}_{m} \bar{\chi}^{\bar{\jmath}} g^{m n}\right) K_{i \bar{\jmath}}\left(\mathcal{D}_{n} A^{i}-\frac{1}{\sqrt{2}} \psi_{n} \chi^{i}\right) \\
& -\frac{1}{2 \sqrt{2}}\left(\psi_{m} \sigma^{n} \bar{\sigma}^{m} \chi^{i}-2 \psi_{m} \chi^{i} g^{m n}\right) K_{i \bar{\jmath}}\left(\mathcal{D}_{n} \bar{A}^{\bar{\jmath}}-\frac{1}{\sqrt{2}} \bar{\psi}_{n} \bar{\chi}^{\bar{\jmath}}\right) .
\end{aligned}
$$

Hence, one obtains for the matter contributions in equation (2.26) and (2.27):

$$
\mathcal{D}_{\text {matter }}=-K_{i \bar{\jmath}} g^{m n} \mathcal{D}_{m} A^{i} \mathcal{D}_{n} \bar{A}^{\bar{\jmath}}-\frac{i}{2} K_{i \bar{\jmath}} \chi^{\alpha i} \sigma_{\alpha \dot{\alpha}}^{m} \mathcal{D}_{m} \bar{\chi}^{\bar{\jmath} \dot{\alpha}}
$$

\footnotetext{
${ }^{2}$ The complex conjugate expressions of the lowest component fields omitted here for brevity.
} 


$$
\begin{aligned}
& +\frac{i}{2}\left(\mathcal{D}_{m} \chi^{\alpha i}\right) \sigma_{\alpha \dot{\alpha}}^{m} K_{i \bar{\jmath}} \bar{\chi}^{\dot{\alpha} \bar{\jmath}}+K_{i \bar{\jmath}} F^{i} \bar{F}^{\bar{\jmath}}-\frac{1}{2} K_{i \bar{\jmath}} g^{m n}\left(\psi_{m} \chi^{i}\right)\left(\bar{\psi}_{n} \bar{\chi}^{\bar{\jmath}}\right) \\
& +\frac{1}{4} R_{i \bar{\jmath} j \bar{\jmath}}\left(\chi^{i} \chi^{j}\right)\left(\bar{\chi}^{\bar{\chi}} \bar{\chi}^{\bar{\jmath}}\right)-\frac{1}{2} K_{i \bar{\jmath}}\left(\chi^{i} \sigma^{a} \bar{\chi}^{\bar{\jmath}}\right) b_{a}+\mathbf{D}^{r} D_{r} \\
& -\frac{1}{\sqrt{2}}\left(\psi_{m} \sigma^{n} \bar{\sigma}^{m} \chi^{i}\right) K_{i \bar{\jmath}} \mathcal{D}_{n} \bar{A}^{\bar{\jmath}}-\frac{1}{\sqrt{2}}\left(\bar{\psi}_{m} \bar{\sigma}^{n} \sigma^{m} \bar{\chi}^{\bar{\jmath}}\right) K_{i \bar{\jmath}} \mathcal{D}_{n} A^{i} \\
& -K_{i \bar{\jmath}}\left[\left(\bar{\psi}_{m} \bar{\sigma}^{m n} \bar{\chi}^{\bar{\jmath}}\right)\left(\psi_{n} \chi^{i}\right)+\left(\psi_{m} \sigma^{m n} \chi^{i}\right)\left(\bar{\psi}_{n} \bar{\chi}^{\bar{\jmath}}\right)\right] \\
& -\sqrt{2} K_{i \bar{\jmath}}\left[\left(\chi^{i} \lambda^{r}\right) \bar{V}_{r}^{\bar{\jmath}}+\left(\bar{\chi}^{\bar{\jmath}} \bar{\lambda}^{r}\right) V_{r}^{i}\right]+\frac{1}{2}\left(\bar{\psi}_{m} \bar{\sigma}^{m} \lambda^{r}-\psi_{m} \sigma^{m} \bar{\lambda}^{r}\right) D_{r}
\end{aligned}
$$

where the covariant derivatives are given by:

$$
\mathcal{D}_{m} A^{k}=\partial A^{k}-a_{m}^{r} V_{r}^{i}
$$

and (see [12])

$$
\begin{aligned}
\mathcal{D}_{m} \chi_{\alpha}^{i} & =\partial_{m} \chi_{\alpha}^{i}-\omega_{m \alpha}{ }^{\gamma} \chi_{\gamma}^{i}-\left(\frac{1}{4} K_{j} \partial_{m} A^{j}-\frac{1}{4} K_{\bar{\jmath}} \partial_{m} \bar{A}^{\bar{\jmath}}\right) \\
& -\frac{i}{4} K_{j \bar{k}}\left(\chi^{j} \sigma_{m} \bar{\chi}^{\bar{k}}\right) \chi^{i}+i a_{m}^{r}\left(i \frac{\partial V_{r}^{i}}{\partial A^{k}}-\frac{1}{2} \delta_{k}^{i} D_{r}\right) \chi_{\alpha}^{k}+\Gamma_{j k}^{i} \chi_{\alpha}^{j} \mathcal{D}_{m} A^{k} \\
\mathcal{D}_{m} \lambda_{\alpha}^{r} & =\partial_{m} \lambda_{\alpha}^{r}-\omega_{m \alpha}^{\beta} \lambda_{\beta}^{r} a_{m}^{q} \lambda_{\alpha}^{s} c_{s q}^{r} \\
& +\left(\frac{1}{4} K_{j} \partial_{m} A^{j}-\frac{1}{4} K_{\bar{\jmath}} \partial_{m} A^{\bar{\jmath}}-\frac{i}{2} e_{m}{ }^{a} b_{\alpha}+\frac{i}{2} a_{m}^{r} D_{r}+\frac{i}{4} K_{i \bar{\jmath}} \chi^{i} \sigma_{m} \bar{\chi}^{\bar{\jmath}}\right) \lambda_{\alpha}^{r} .
\end{aligned}
$$

In the above equations, $D_{r}$ is the Killing potential, not to be confused with the gauge auxiliary field $\mathbf{D}_{r}=-\frac{1}{2} \mathcal{D}^{\alpha} \mathcal{W}_{\alpha}^{r} \mid$.

The supersymmetry transformations of the component fields can be derived by starting with the superspace transformation laws of the supervielbein and a (Lorentz invariant) generic field $\Gamma$ as given in eq. (2.22) with

$$
\xi^{a}\left|=0, \quad \xi^{\alpha}\right|=\xi^{\alpha}, \quad \xi_{\dot{\alpha}} \mid=\xi_{\dot{\alpha}}
$$

this is a laborious procedure and has been worked out to great detail in ref. [12]. For instance, the lowest component of the transformation of the supergravity multiplet gives:

$$
\begin{aligned}
\delta e_{m}^{a} & =i \xi \sigma^{a} \bar{\psi}_{m}+i \bar{\xi} \bar{\sigma}^{a} \psi_{m} \\
\delta \psi_{m}^{\alpha} & =2 \mathcal{D}_{m} \xi^{\alpha}-\frac{i}{3}\left(\xi \sigma^{a} \bar{\sigma}_{m}\right)^{\alpha} b_{a}+\frac{i}{3}\left(\bar{\xi} \bar{\sigma}_{m}\right)^{\alpha} M \\
& -\frac{1}{2 \sqrt{2}} \psi_{m}^{\alpha}\left(K_{i} \xi \chi^{i}-K_{\bar{\imath}} \bar{\xi} \bar{\chi}^{\bar{\imath}}\right)
\end{aligned}
$$

where $\mathcal{D}_{m} \xi^{\alpha}=\partial_{m} \xi^{\alpha}+\xi^{\beta} \omega_{m \beta}{ }^{\alpha}+\xi^{\alpha} \tilde{A}_{m}$ and the Kähler connection $\tilde{A}_{m}=\frac{1}{4} K_{j} \partial_{m} A^{j}-$ $\frac{1}{4} K_{\bar{\jmath}} \partial_{m} A^{\bar{j}}-\frac{i}{2} e_{m}{ }^{a} b_{a}+\frac{i}{2} a_{m}^{r} D_{r}+\frac{i}{4} K_{i \bar{\jmath}} \chi^{i} \sigma_{m} \bar{\chi}^{\bar{\jmath}}$. The transformations of the auxiliary components, and all the other component fields in the theory are found in [12]. 


\section{Construction of An InVARIANT ACTION}

Given a chiral superfield $\mathbf{r}$ with $w(\mathbf{r})=2$ (which cancels the Kähler weight of the $\theta$ integration) and components:

$$
\mathbf{r}\left|=r, \quad \frac{1}{\sqrt{2}} \mathcal{D}_{\alpha} \mathbf{r}\right|=\rho_{\alpha}, \quad-\frac{1}{4} \mathcal{D}^{\alpha} \mathcal{D}_{\alpha} \mathbf{r} \mid=s .
$$

The supersymmetry transformation laws for the components of $\mathbf{r}$ can be found and they are given by:

$$
\begin{aligned}
\delta r & =\sqrt{2} \xi^{\alpha} \rho_{\alpha}-\frac{1}{\sqrt{2}}\left(K_{i} \xi \chi^{i}-K_{\bar{\imath}} \bar{\xi} \bar{\chi}^{\bar{\imath}}\right) \\
\delta \rho_{\alpha} & =\sqrt{2} \xi^{\alpha} s+i \sqrt{2}\left(\sigma^{m} \bar{\xi}\right)_{\alpha}\left(\mathcal{D}_{m} r-\frac{1}{\sqrt{2}} \psi_{m} \rho+i e_{m}{ }^{a} b_{a} r\right) \\
& -\frac{1}{2 \sqrt{2}} \rho_{\alpha}\left(K_{k} \xi \chi^{k}-K_{\bar{k}} \bar{\xi} \bar{\chi}^{\bar{k}}\right) \\
\delta s & =-i \sqrt{2}\left(\bar{\xi} \bar{\sigma}^{m}\right)^{\alpha} \mathcal{D}_{m} \rho_{\alpha}-i\left(\bar{\xi} \bar{\sigma}^{m} \psi_{m}\right) s+\left(\bar{\xi} \bar{\sigma}^{m} \sigma^{n} \psi_{m}\right)\left(\mathcal{D}_{n} r-\frac{1}{\sqrt{2}} \psi_{n} \rho+i e_{n}{ }^{a} b_{a} r\right) \\
& +\frac{\sqrt{2}}{3} \bar{M}(\xi \rho)-\frac{2 \sqrt{2}}{3}\left(\bar{\xi} \bar{\sigma}^{m} \rho\right) b_{a}+2 r \bar{\xi}_{\dot{\alpha}} \bar{X}^{\dot{\alpha}} .
\end{aligned}
$$

These can be used to show that the Lagrangian density

$$
\mathcal{L}=e\left[s+\frac{i}{\sqrt{2}}\left(\bar{\psi}_{m} \bar{\sigma}^{m} \rho\right)-r\left(\bar{M}+\bar{\psi}_{m} \bar{\sigma}^{m n} \bar{\psi}_{n}\right)\right]+\text { h.c. }
$$

(where $e=\operatorname{det} e_{m}{ }^{a}$ ) has the desired transformation property that $\delta \mathcal{L}$ is a total divergence, and therefore is a supersymmetric action:

$$
\delta \mathcal{L}=\partial_{m}\left(i \sqrt{2} e\left(\bar{\xi} \bar{\sigma}^{m} \rho\right)-4 e\left(\bar{\xi} \bar{\sigma}^{m n} \bar{\psi}_{n}\right) r\right)+h . c .
$$

Referring back to the discussion of the superfield action for supergravity, SYM and matter, with superpotential $W$, where we have:

$$
\mathbf{r}=-3 R+e^{K} W+\frac{1}{4} f_{r s} \mathcal{W}^{\alpha r} \mathcal{W}_{\alpha}^{s}
$$

Recall from equations (2.26)-(2.29) that the first term gives the $\mathcal{L}_{\text {sugra+matter }}$ with the canonically normalized Einstein term, and the second and third terms give $\mathcal{L}_{\text {sp }}$ and $\mathcal{L}_{\mathrm{YM}}$. For the sake of an example, let us consider the construction of $\mathcal{L}_{\text {sp }}$ from the second term in (2.48); i.e., let us start with $\mathbf{r}=e^{K} W$ which gives:

$$
\mathbf{r}\left|=r=\left(e^{K} W\right)\right|=e^{K} W,
$$




$$
\begin{aligned}
\rho_{\alpha} & =\frac{1}{\sqrt{2}} \mathcal{D}_{\alpha} \mathbf{r}\left|=\frac{1}{\sqrt{2}}\left[e^{K / 2}\left(K_{k} W+W_{k}\right) \mathcal{D}_{\alpha} \varphi^{k}\right]\right| \\
& =e^{K / 2}\left(K_{k} W+W_{k}\right) \chi_{\alpha}^{k} \\
s & =-\frac{1}{4} \mathcal{D}^{\alpha} \mathcal{D}_{\alpha} \mathbf{r} \mid=e^{K / 2}\left(K_{k} W+W_{k}\right) F^{k}-\frac{1}{2} e^{K / 2}\left(K_{i j}-K_{\ell} \Gamma_{i j}^{\ell}+K_{i} K_{j}\right) W \\
& -\frac{1}{2} e^{K / 2}\left(W_{i j}-W_{k} \Gamma_{i j}^{k}+2 W_{i} K_{j}\right)\left(\chi^{i} \chi^{j}\right)
\end{aligned}
$$

which using (2.46) gives:

$$
\begin{aligned}
\frac{1}{e} \mathcal{L}_{\mathrm{sp}} & =e^{K / 2}\left[F^{k}\left(K_{k} W+W_{k}\right)-M \bar{W}+\frac{i}{\sqrt{2}}\left(\bar{\psi}_{m} \bar{\sigma}^{m} \chi^{k}\right)\left(K_{k} W+W_{k}\right)\right. \\
& -\frac{1}{2}\left[\left(K_{i j}-K_{\ell} \Gamma_{i j}^{\ell}+K_{i} K_{j}\right) W+\left(W_{i j}-W_{k} \Gamma_{i j}^{k}+2 W_{i} K_{j}\right)\right]\left(\chi^{i} \chi^{j}\right) \\
& \left.-\bar{\psi}_{m} \bar{\sigma}^{m n} \bar{\psi}_{n} W\right]+ \text { h.c. }
\end{aligned}
$$

$\mathcal{L}_{\text {sugratmatter }}$ and $\mathcal{L}_{\mathrm{YM}}$ are similarly obtained are are given by:

$$
\begin{aligned}
\mathcal{L}_{\text {sugra+matter }} & =-\frac{1}{2} \mathcal{R}+\frac{1}{2} \epsilon^{m n p q}\left(\bar{\psi}_{m} \bar{\sigma}_{n} \mathcal{D}_{p} \psi_{q}-\psi_{m} \sigma_{n} \mathcal{D}_{p} \bar{\psi}_{q}\right) \\
& -\frac{1}{3} M \bar{M}+\frac{1}{3} b^{a} b_{a}+\mathcal{D}_{\text {matter }}
\end{aligned}
$$

where $\mathcal{D}_{\text {matter }}$ was given in eq. (2.40) containing the complete matter dependence other than the superpotential. The YM Lagrangian is:

$$
\begin{aligned}
\frac{1}{e} \mathcal{L}_{\mathrm{YM}}= & -\frac{1}{4} f_{\tau s}\left[\frac{1}{2} F^{r m n} F_{m n}^{s}+\frac{i}{4} \epsilon^{m n p q} F_{m n}^{r} F_{p q}^{s}+2 i \lambda^{r} \sigma^{m} \mathcal{D}_{m} \bar{\lambda}^{s}\right. \\
& -\mathbf{D}^{r} \mathbf{D}^{s}-\left(\lambda^{r} \sigma^{a} \lambda^{s}\right) b_{a}-i F^{r m n}\left(\psi_{m} \sigma_{n} \bar{\lambda}^{s}+\bar{\psi}_{m} \bar{\sigma}_{n} \lambda^{s}\right) \\
& +\frac{1}{2} \epsilon^{m n p q} F_{m n}^{r}\left(\psi_{p} \sigma_{q} \bar{\lambda}^{s}-\bar{\psi}_{p} \bar{\sigma}_{q} \lambda^{s}\right)+\frac{1}{8}\left(\lambda^{r} \lambda^{s}\right)\left(3 \bar{\psi}_{m} \bar{\psi}^{m}+2 \bar{\psi}_{m} \bar{\sigma}^{m n} \bar{\psi}_{n}\right) \\
& +\frac{1}{8}\left(\bar{\lambda}^{r} \bar{\lambda}^{s}\right)\left(3 \psi_{m} \psi^{m}+2 \psi_{m} \bar{\sigma}^{m n} \psi_{n}\right) \\
& \left.-\frac{1}{2}\left(g^{m p} g^{n q}-g^{m q} g^{n p}-i \epsilon^{m n p q}\right)\left(\bar{\psi}_{m} \bar{\sigma}_{n} \lambda^{r}\right)\left(\psi_{m} \sigma_{n} \bar{\lambda}^{r}\right)\right] \\
& -\frac{1}{4} \frac{\partial f_{r s}}{\partial A^{i}}\left[\sqrt{2}\left(\chi^{i} \sigma^{m n} \lambda^{r}\right) F_{m n}^{s}-i \sqrt{2}\left(\chi^{i} \lambda^{r}\right) \mathbf{D}^{s}+F^{i}\left(\lambda^{r} \lambda^{s}\right)\right. \\
& \left.-i \frac{\sqrt{2}}{4}\left(\lambda^{r} \lambda^{s}\right)\left(\bar{\psi}_{m} \bar{\sigma}^{m} \chi^{i}\right)-i \sqrt{2}\left(\psi_{m} \sigma_{n} \bar{\lambda}^{r}\right)\left(\chi^{i} \bar{\sigma}^{m n} \lambda^{s}\right)\right] \\
& +\frac{1}{8}\left[\frac{\partial^{2} f_{r s}}{\partial A^{i} A^{j}}-\Gamma_{i j}^{\ell} \frac{\partial f_{r s}}{\partial A^{\ell}}\right]\left(\chi^{i} \chi^{j}\right)\left(\lambda^{r} \lambda^{s}\right)+\text { h.c. }
\end{aligned}
$$

In the above equations, $F_{m n}^{r}=\partial_{m} a_{n}^{r}-\partial_{n} a_{m}^{r}-c_{s t}^{r} a_{m}^{s} a_{n}^{t}$ are the bosonic components of the YM gauge field strength. Solving for the auxiliary fields in the Lagrangians (2.37), $(2.48)-(2.50)$ we find:

$$
M=-3 e^{K / 2} W
$$




$$
\begin{aligned}
b^{a} & =\frac{3}{4} K_{i \bar{\jmath}}\left(\chi^{i} \sigma^{a} \bar{\chi}^{\bar{\jmath}}\right)-\frac{3}{4} \operatorname{Re} f_{r s}\left(\lambda^{r} \sigma^{a} \bar{\lambda}^{s}\right) \\
F^{i} & =-e^{K / 2} K^{i \bar{\jmath}}\left(K_{\bar{\jmath}} \bar{W}+\bar{W}_{\bar{\jmath}}\right)+\frac{1}{4}\left(\bar{\lambda}^{r} \bar{\lambda}^{s}\right) K^{-i \bar{\jmath}} \frac{\partial \bar{f}_{r s}}{\partial \bar{A}^{\bar{\imath}}} \\
\mathbf{D}^{r} & =\frac{1}{\operatorname{Re} f_{r s}}\left(-i \frac{\sqrt{2}}{4}\left[\frac{\partial f_{s t}}{\partial A^{i}} \lambda^{t} \chi^{i}-\frac{\partial \bar{f}_{s t}}{\partial \bar{A}^{\overline{2}}} \bar{\lambda}^{t} \bar{\chi}^{\bar{\imath}}\right]-D_{s}\right) .
\end{aligned}
$$

It can be verified that these equations are precisely the lowest components of the equations of motion of the superfield action of (2.26)-(2.29).

\subsection{The Heterotic String and Compactifications}

In this section, we shall briefly review some relevant facts about heterotic string, it's effective field theory in 10 dimensions, and the compactifications to four dimensions. We shall not go into any details of the worldsheet theory because we are concerned with the physics in the low energy effective field theory in four dimensions.

It is well known that the negative-norm ghost states of the 26 dimensional bosonic string theory are removed by world-sheet supersymmetry [14]. This supersymmetric theory has ten spacetime dimensions. In addition, in the spacetime supersymmetric theory the so called GSO projection [15] eliminates the potential tachyonic ground state.

If all the heavy modes of the superstring are 'integrated out' (or in practice, the theory is truncated which means the massive modes are discarded), the effective theory is described by the massless modes in ten dimensions. For example, anticipating the case of heterotic theory (with one supersymmetry) these modes are:

- $g_{M N}$, Th 10-dimensional metric (symmetric and traceless) corresponding to the graviton. In terms of the string states, this mode is given by: ${ }^{3}$

$$
\left(\alpha_{-1}^{M} \tilde{\alpha}_{-1}^{N}+\alpha_{-1}^{N} \tilde{\alpha}_{-1}^{M}-\delta_{M N} \alpha_{-1}^{M} \tilde{\alpha}_{-1}^{N}\right)|0\rangle
$$

- $B_{M N}$ Antisymmetric tensor, corresponding to:

$$
\left(\alpha_{-1}^{M} \tilde{\alpha}_{-1}^{N}-\alpha_{-1}^{N} \tilde{\alpha}_{-1}^{M}\right)|0\rangle
$$

- $\varphi$, Scalar field (dilaton) corresponding to:

$$
\delta_{M N}\left(\alpha_{-1}^{M} \tilde{\alpha}_{-1}^{N}\right)|0\rangle
$$

\footnotetext{
${ }^{3} \alpha_{m}^{M}$ and $\tilde{\alpha}_{n}^{N}$ are the oscillators in the mode expansions of the string right- and left-moving coordinates, $X_{R}^{\mu}$, and $X_{L}^{\mu}$, and $m, n \leq-1$ correspond to the creation operators in the Fock space of the string states [14].
} 
and their superpartners, which are gravitino $\psi_{M}$, Majorana fermion $\chi$ (dilatino). To the above fields one must add the YM gauge potential $A_{M}^{r}$ and gaugino $\lambda^{r}$.

These are exactly the contents of the physical spectrum of $10 \mathrm{D}$ supergravity coupled to YM gauge theory. Ten dimensional supergravities are thus the low energy limits of superstring theories:

The field contents of type I, type IIA, and type IIB supergravities in ten dimensions corresponding to open string with $N=1$ susy (I), and closed superstring with $N=2$ supersymmetries in which the left and right moving fermions are of opposite (IIA) and the same (IIB) chiralities are as follows:

Type I:

bosons: Graviton, dilaton, antisymmetric rank 2 tensor, YM vector potential, fermions: Gravitino (spin 3/2), spinor (dilatino), gaugino.

Type IIA:

bosons: Graviton, dilaton, antisymmetric rank 2 tensor, vector, antisymmetric rank 3 tensor,

fermions: 2 Gravitinos(with opposite chiralities), 2 spinors,

Type IIB:

bosons: Graviton, dilaton, antisymmetric rank 2 tensor, 2 scalars, self-dual antisymmetric rank 4 tensor, fermions: 2 Gravitinos (with the same chirality), 2 spinors,

The presence of the antisymmetric tensors in the 10-D supergravities has far reaching consequences in the developments in string-string dualities [16].

\section{The Heterotic String}

The heterotic string [17] is constructed using the fact that in closed string theories, left- and right-moving modes are decoupled, and the left-moving modes can be of different type from the right-moving modes. In particular, one can take the rightmoving modes to be the superstring modes (this introduces supersymmetry and therefore fermions, and also removes the tachyon), while taking the left-moving modes to be the bosonic string modes and left-moving fermions which arise from the fermionization of 
the extra left-moving bosons. More explicitly, the action that describes this type of construction is as follows:

$$
S=\frac{-1}{2 \pi} \int d^{2} \sigma\left(\sum_{\mu=0}^{9}\left(\partial_{\alpha} X^{\mu} \partial^{\alpha} X_{\mu}+2 \psi_{+}^{\mu} \partial_{-} \psi_{\mu+}\right)-2 \sum_{A=1}^{n} \lambda_{-}^{A} \partial_{+} \lambda_{-}^{A}\right)
$$

where $\partial \pm=\frac{1}{2}\left(\frac{\partial}{\partial \tau} \pm \frac{\partial}{\partial \sigma}\right)$, and $\tau$ and $\sigma$ are the time and spatial coordinates of the worldsheet. The fields $\psi_{\mu}$ and $\lambda^{A}$ are Majorana-Weyl fermions. The right-moving parts of $X^{\mu}$ together with $\psi_{+}^{\mu}$ are the right-moving modes with critical dimension. $D=10$. The right moving fields have supersymmetry: $\delta X^{\mu}=\epsilon \psi_{+}^{\mu}$ and $\delta \psi_{+}^{\mu}=\epsilon \partial_{+} X^{\mu}$ where $\epsilon$ has + chirality only. The left-moving modes are the left-moving parts of $X^{\mu}$ and the fermions $\lambda^{A}$. The latter are singlets under the Lorentz group, and there is no supersymmetry amongst the left-movers. Depending on whether all $\lambda^{A}$ obey the same boundary condition, they carry an $S O(32)$ or $E_{8} \times E_{8}$ symmetry, which indeed turns out to be a gauged symmetry (for discussion, see for example [14]).

To give a feeling for how the gauge symmetry arises, let us simply remark that because the right-movers are in 10 spacetime dimensions, while the left-movers are in 26 dimensions, 16 of the left-mover coordinates, $X_{L}^{\mu}$ are toroidally compactified on a 16 dimensional torus, giving rise to extra massless states which turn out to be vectorlike, and are associated with gauge fields of $S O(32)$ or $E_{8} \times E_{8}$. The details are found in standard references $[14,18,19]$. Remarkably, anomaly cancellation in the ten dimensional supergravity and superYM theory requires precisely the choice of $S O(32)$ or $E_{8} \times E_{8}$ gauge groups [20].

The ten dimensional effective field theory of heterotic string theory is $N=1$ supergravity coupled to super-YM in ten dimensions. This is because the truncation of the theory (i.e., discarding all the string modes except the massless ones) leaves us with the following spectrum:

$$
\begin{aligned}
\text { bosons : } & 8_{V}^{L} \times\left(8_{V}+1 \operatorname{adj}(G)\right)^{R}=1+28+35_{V}+8_{V} \operatorname{adj}(G) \\
\text { fermions : } & 8_{C}^{L} \times\left(8_{V}+1 \operatorname{adj}(G)\right)^{R}=8_{S}+56_{S}+8_{C} \operatorname{adj}(G)
\end{aligned}
$$

and $(1+28+35 \mathrm{~V})_{\text {Bose }}$ (corresponding to a scalar, second rank tensor, and the graviton, respectively) $+\left(8_{S}+56_{S}\right)_{\text {Fermi }}$ (corresponding to a Majorana-Weyl fermion, and gravitino, respectively) constitute the $N=1$ supergravity multiplet in 10 dimensions and $\left(8_{V}+8_{C}\right) a d j(G)$ correspond to the super-YM multiplet, where $G$ is the either the $E_{8} \times E_{8}$ or the $S O(32)$ gauge group, which are both rank 16, and 248 dimensional groups. 
This should make the phenomenological appeal of the heterotic string apparent. Type IIA is unsuitable because it is non-chiral, and type IIB has the undesirable feature that all of its massless states are contained in the $N=2$ supergravity multiplet, therefore there are no nonabelian gauge fields in the effective 10-dimensional field theory. Type $I$ is also unsuitable because the gauge group ( $S O(32))$ does not admit any complex representations. Heterotic string on the other hand has an effective theory which accommodates chiral fermions and also provides a large gauge symmetry which can contain the gauge structure of the Standard Model.

\section{COMPACTIFICATION FROM 10D DOWN TO 4D}

The different string models in four dimensions correspond to the choice of the compact space $\mathcal{K}$ in the decomposition of the $10 \mathrm{~d}$ spacetime $\mathcal{R}^{10}=\mathcal{R}^{4} \times \mathcal{K}$ and on the choice of boundary conditions imposed along the compactified directions. Let us start with the simplest case in which we compactify only one direction on a circle of radius $R$, i.e., $\mathcal{R}^{10}=\mathcal{R}^{9} \times S^{1}$. Then along the compact direction the momenta are given by:

$$
p_{R}=\frac{m}{2 R}-n R \quad p_{L}=\frac{m}{2 R}+n R
$$

and mass formula $M^{2}=\frac{m^{2}}{4 R^{2}}+n^{2} R^{2}+\left(N_{R}+N_{L}-2\right)$ where $m$ and $n$ are integers corresponding to the quantization of momenta along the compact direction and the winding of the string around the circle $n$ times, respectively. Notice that the latter is purely of stringy origin. The mass formula exhibits another stringy property, namely the invariance of the spectrum under the duality transformation:

$$
m \longleftrightarrow n, \quad R \longleftrightarrow \frac{1}{2 R} .
$$

This is a manifestation of modular invariance in string theory about which we shall say more in this and subsequent chapters. It exchanges, simultaneously, the Kaluza-Klein momentum states with the winding states and large $R$ with small $R$. Modular symmetry holds to all orders of perturbation in string theory.

\section{Toroidal Compactification}

In compactifying supergravity theories from ten dimensions to a lower dimension, the general scenario is that one ends up with extended supersymmetries. This is certainly 
the case in toroidal compcatifications which generically leads to $N=4$ theories in four dimensions [14]. However, in order to obtain a chiral model in four dimensions, and to make contact with phenomenology, at most $N=1$ should be required. However, we can still learn some lessons from considering the relatively simple case of toroidal compactification. Especially, since they are in some sense fundamental to the interesting case of orbifold compactification. We shall see shortly that requiring $N=1$ supersymmetry in four dimensions puts certain constraints on the compact 6 -dimensional manifold $\mathcal{K}$. But before that, we consider the compactification on $T^{2}$.

We identify the compact dimensions by vectors of the defining lattice of the 2-torus. The compact metric has three independent components: $g_{M N} \rightarrow g_{m n}, g_{11}, g_{12}, g_{22}$ and the antisymmetric tensor has only one independent 'compact component': $B_{M N} \rightarrow$ $B_{m n}, B_{12}$. From the compact components of these we can build two complex moduli fields:

$$
\begin{aligned}
& U=\frac{g_{12}}{g_{22}}+i \frac{\sqrt{g}}{g_{22}} \\
& T=B_{12}+i \sqrt{g}
\end{aligned}
$$

where $g$ is the determinant of the $2 \mathrm{D}$ metric on the torus $\left(=g_{11} g_{22}-g_{12}^{2}\right)$. $U$ is usually referred to as the 'complex structure modulus', and $T$ as the 'Kähler modulus'. Note that $\operatorname{Im} T=\sqrt{g}$ gives the size of the torus. Here the left- and right-moving momenta are:

$$
\begin{aligned}
& p_{L}^{2}=\frac{1}{2 \operatorname{Im} T \operatorname{Im} U}\left|\left(n_{1}-n_{2} U\right)-T\left(m_{2}+m_{1} U\right)\right|^{2} \\
& p_{R}^{2}=\frac{1}{2 \operatorname{Im} T \operatorname{Im} U}\left|\left(n_{1}-n_{2} U\right)-\bar{T}\left(m_{2}+m_{1} U\right)\right|^{2} .
\end{aligned}
$$

Once again, the mass formula which depends on $p_{R}^{2}+p_{L}^{2}$ is invariant under a modular symmetry, in this case under two copies of $S L(2, \mathcal{Z})$ :

$T$-modular transformations: $T \longrightarrow \frac{a T+b}{c T+d}, a d-b c=1$,

$U$-modular transformations: $U \longrightarrow \frac{a^{\prime} U+b^{\prime}}{c^{\prime} U+d^{\prime}}, a^{\prime} d^{\prime}-b^{\prime} c^{\prime}=1$.

The T-duality is the analogue of the large-small radius duality in the previous case. It also requires exchanging the momentum quantum numbers $m_{1}$ and $m_{2}$ with the winding numbers $n_{1}$ and $n_{2}$. The duality symmetries imply that $T$ and $U$ each live in the fundamental domain of the complex plane [14]. In other words, the space of these moduli is not the product of two complex planes $S L(2, \mathcal{R}) / O(2) \times S L(2, \mathcal{R}) / O(2) \cong O(2,2, \mathcal{R}) /(O(2) \times$ 
$O(2)$, but the subset with which all the points of the product complex space are identified under the action of the duality group $S L(2, \mathcal{Z}) \times S L(2, \mathcal{Z}) \cong O(2,2, \mathcal{Z})$.

For the $d$-dimensional torus, the above result immediately generalizes to the following. The moduli space of the torus is $\mathcal{M}=O(d, d, \mathcal{R}) / O(d) \times O(d)$ with points identified by the duality group, $O(d, d, \mathcal{Z})$. In particular for the heterotic string which has 16 extra left-moving coordinates, the moduli space is $\mathcal{M}=O(d+16, d, \mathcal{R}) / O(d+16) \times O(d)$ with points identified under the action of the duality group $O(d+16, d, \mathcal{Z})$. In this case, for $d=6$ (six dimensional torus) $p_{L}$ and $p_{R}$ are defined on the so-called Narain lattice [21], an even, self-dual lattice $\Lambda_{22,6}$ (a generalization of the $\Lambda_{2,2}$ lattice of the $T^{2}$ compactification, characterized by the integers $m_{1}, m_{2}, n_{1}, n_{2}$ of eq. (2.64)). The dimension of the moduli space of the $d$-torus on which the heterotic string is compactified is $d(d+16)$ or 132 for $T^{6}$. This corresponds exactly to the number of independent components of $g_{m n}$, $B_{m n}$, and $A_{m}^{I}(m, n=1, \cdots, 6$, and $I=1, \cdots, 16)$.

As noted above, toroidal compactifications result in extended supersymmetries [14] in 4 dimensions (generically, $N=4$ ). But the lessons which can be learned from them, for our purposes, are the presence of modular symmetry amongst the moduli, and that the latter are expected to show up as degrees of freedom in the four dimensional compactified theory.

Requiring $N=1$ supersymmetry in the $4 \mathrm{D}$ theory turns out to be somewhat restrictive on the compact space. It can be shown that the necessary and sufficient condition for one supersymmetry in four dimensions is that the compact 6-fold must have holonomy group $S U(3)$ (or equivalently, has vanishing first Chern class), which in turn implies that the compact 6-fold is a Kähler manifold with $R_{i j}=0$ (for details, see [22] or [14]). These manifolds have been studied extensively, and are called Calabi-Yau (CY) manifolds. Compactification of the $E_{8} \times E_{8}$ heterotic string on a CY manifold yields the gauge group $E_{6} \times E_{8}$ in the $4 \mathrm{D}$ theory [22]. We shall not discuss CY compactification in any further detail here. Instead, we turn to orbifold compactifications which are easier to study phenomenologically [23].

\section{ORBIFOLD COMPACTIFICATION}

Actually, orbifolds can be thought of as singular limits of CY manifolds. In our discussion of compactification, we have so far not considered the possibility of twists in the boundary conditions along the compact directions, although we have used identifica- 
tions corresponding to the shift symmetries of the lattice defining the underlying torus of compactification. For instance, if in the 2-torus example, we identify $X^{i}$ and $-X^{i}$ (i.e., $\bmod$ out by $\mathcal{Z}_{2}$ corresponding to a rotation by $\pi$ ), we end up with the orbifold $T^{2} / \mathcal{Z}_{2}=\mathcal{R}^{2} / \Lambda_{2} / \mathcal{Z}_{2}$, with four singularities at the fixed-points of the rotation. In order to be explicit, let us discuss the case of the heterotic string compactification on a $\mathcal{Z}_{3}$ orbifold to four dimensions. In this case, the point group (discrete group of rotations) is $\mathcal{Z}_{3}$ acting on the lattice of the underlying 6-torus. Let us take the following complex coordinates for the six-dimensional compact manifold:

$$
\begin{aligned}
& Z^{1}=\frac{1}{\sqrt{2}}\left(Y^{3}+i Y^{4}\right) \\
& Z^{2}=\frac{1}{\sqrt{2}}\left(Y^{5}+i Y^{6}\right) \\
& Z^{3}=\frac{1}{\sqrt{2}}\left(Y^{7}+i Y^{8}\right) .
\end{aligned}
$$

The lattice for the underlying torus is defined by identifying $(\alpha=1,2,3)$ :

$$
Z^{\alpha} \equiv Z^{\alpha}+1
$$

and

$$
Z^{\alpha} \equiv Z^{\alpha}+e^{2 \pi i / 3}
$$

i.e.,

$$
\vec{Z} \equiv \vec{Z}+\sum_{\lambda=1}^{3}\left(m_{\lambda} \vec{e}_{\lambda}+n_{\lambda} \vec{f}_{\lambda}\right)
$$

where $m_{\lambda}$ and $n_{\lambda}$ are integers, and the basis vectors of the lattice are defined as follows:

$$
\vec{e}_{1}=(1,0,0), \quad \vec{e}_{2}=(0,1,0), \quad \vec{e}_{3}=(0,0,1), \quad \vec{f}_{\lambda}=e^{2 \pi i / 3} \vec{e}_{\lambda}
$$

The generator for the point group is:

$$
\Omega=\operatorname{diag}\left(e^{2 \pi i / 3}, e^{2 \pi i / 3}, e^{2 \pi i / 3}\right)
$$

The $S O(6)$ rotation group (acting on the real coordinates $Y^{k}, k=3 \cdots 8$ ) has a subgroup $S U(3)$. The coordinates $\vec{Z}$ provide a 3 -dimensional representation of this $S U(3)$, and $\Omega$ is a finite element this $S U(3)$. Note also the action of $\Omega$ on the basis vectors of the lattice

$$
\Omega \vec{e}_{\lambda}=\vec{f}_{\lambda}, \quad \Omega \vec{f}_{\lambda}=-\vec{e}_{\lambda}-\vec{f}_{\lambda}
$$


Thus the discrete group generated by $\Omega$ is a symmetry of the torus. The orbifold is constructed by identifying the points on the torus that are mapped into one another under the point group generated by $\Omega$. The fixed points of the combined action of $\Omega$ and lattice translations by $\sum_{\lambda=1}^{3}\left(m_{\lambda} \vec{e}_{\lambda}+n_{\lambda} \vec{f}_{\lambda}\right)$ turn out to be of the form:

$$
\vec{Z}=\frac{e^{2 \pi i / 3}}{\sqrt{3}}\left(k_{1}, k_{2}, k_{3}\right), \quad k_{\lambda}=0, \pm 1, \quad(\lambda=1, \cdots, 3)
$$

hence there are 27 fixed points (singularities) altogether. As mentioned earlier, $\Omega$ is in fact a finite element of $S U(3)$. If we associate with $\Omega$ an action on the gauge degrees of freedom, for example, by taking $\Omega$ to be an element of $S U(3)$ subgroup of an $E_{6} \times$ $S U(3) \times E_{8}^{\prime} \subset E_{8} \times E_{8}^{\prime}$ (this is called the 'standard embedding') the theory turns out to be at the tree-level modular invariant [14]. The states of the toroidally compactified theory do not necessarily correspond to the states of the orbifold, because one has to select the states that are invariant under the action of the point group, and its embedding in the gauge group. Even then, these point group invariant states are only a part of the story, they correspond to the 'untwisted sector'. These are obviously a subset of the states of the underlying toroidal compactification. The additional states, or the 'twisted sector', are obtained by imposing twisted boundary conditions, i.e., satisfying boundary conditions along the compact directions up to the action of the point group (in our case, rotation by $2 \pi / 3)$. Let us now briefly discuss the massless states. It can be shown $[18,23]$ that point group invariant compactified theory indeed has $N=1$ supersymmetry. The embedding of the point group into the gauge group reduces one of the $E_{8}$ factors to $E_{6} \times S U(3)$. The adjoint representation of $E_{8}$ (which is 248 dimensional) decomposes under $E_{6} \times S U(3)$ as:

$$
248=(78,1)+(1,8)+(27,3)+(\overline{27}, \overline{3})
$$

and requiring point group invariant states eliminates the $(\mathbf{2 7}, \mathbf{3})$ and $(\overline{\mathbf{2 7}}, \overline{\mathbf{3}})$ and leaves only the adjoint of $E_{6},(\mathbf{7 8}, \mathbf{1})$, and the adjoint of $S U(3),(\mathbf{1}, \mathbf{8})$. Note that $\mathbf{8}$ is $\Omega$ invariant because it is contained in $\mathbf{3} \times \overline{\mathbf{3}}$, and $\mathbf{3}$ and $\overline{\mathbf{3}}$ have opposite phases under $\Omega$ transformation. The matter fields in the compactified theory are obtained from rightmovers which transform as 3 of the $S U(3)$ subgroup of $S O(6)$ of the spatial degrees of freedom of the compact manifold, together with the left-movers in $(\overline{\mathbf{2 7}}, \overline{\mathbf{3}})$. The former transform with a phase $e^{2 \pi i / 3}$ and the latter with $e^{-2 \pi i / 3}$ under $\Omega$, and so these are point group invariant. Likewise, right-movers which transform as $\overline{\mathbf{3}}$ of $S U(3)$ are linked with 
the left-movers in $(\mathbf{2 7}, \mathbf{3})$. In this way, three copies of point group invariant $\mathbf{2 7}$ and $\overline{\mathbf{2 7}}$ of $E_{6}$ are generated (one for each index $\alpha$ in (2.66). These are three copies of chiral superfields in $(27,3)$ of $E_{6} \times S U(3)$ and their antiparticles. In addition, in the untwisted sector there are a set of point group invariant $E_{6}$-singlets (moduli) whose expectation values are related to the size and shape of the underlying torus. There are two 'twisted sectors' of the $\mathcal{Z}_{3}$ orbifold compactification which are not discussed here. The $E_{6}$ gauge symmetry of the heterotic string's observable sector (and likewise, the hidden $E_{8}$ ) can be further broken and additional matter can be generated by adding Wilson lines [23, 24] which amounts to the full embedding of the lattice translations (of the underlying $6 \mathrm{D}$ torus) as well as the rotations by the point group in the gauge degrees of freedom. This further increases the possible consistent models based on heterotic strings. 


\section{Chapter 3}

\section{Supergravity Coupled to YM and Matter at One Loop}

\subsection{Introduction}

Understanding the structure of the divergences in supergravity is a necessary step in determining the counterterms [25], [26], [29] that are needed to fully restore modular invariance in an effective supergravity theory from superstrings. The determination of these loop corrections may also provide a guide to the construction of an effective theory for a composite chiral multiplet that is a bound state of strongly coupled Yang-Mills superfields, which in turn could shed light on gaugino condensation as a mechanism for supersymmetry breaking.

The paper [30] (hereafter referred to as I), gives the divergent contributions to the bosonic Lagrangian in a general supergravity theory coupled to chiral matter, in a general bosonic background, averaged over quantum fermion helicities. That work extended the results of several earlier calculations [37]-[40] on loop corrections to supergravity. In particular, using specific choices of the gauge fixing and of the expansion of the action, the authors were able to cast the results in an especially simple form in which most of the one-loop corrections can be interpreted in terms of renormalizations. More recently, the authors of I and myself extended and completed these results to incorporate the Yang-Mills sector [41], including helicity-odd operators that arise from integration over quantum fermions. This work appears in ref. [31] (hereafter referred to as II) and is combined with the results of $I$ in a short letter in [32]. Our results are completely general, except that we assume that the tree-level gauge kinetic energy normalization function $f(z)$ [42], where $z$ represents the complex scalar fields of the theory, is proportional to the unit matrix. This is the case for all known theories derived from superstrings, up to possible multiplicative constants for different factor gauge groups that correspond to higher affine levels [43]. This modification is easily incorporated into our formalism, as explained in Section 4.5 .

The generalization of the results of $I$ to the more general case considered here can be summarized as follows. We define an operator of dimension $d$ as a Kähler invari- 
ant operator whose term of lowest dimension is $d$, where scalar and Yang-Mills fields are assigned the canonical dimension of unity. Then, among the ultra-violet divergent terms generated at one loop, all operators of dimension 6 or less (as well as many operators of dimension 8) that involve neither the Kähler curvature nor derivatives of the gauge kinetic function can be absorbed by field redefinitions, interpreted as renormalizations of the Kähler potential, or take the form $F_{a b}(z, \bar{z})\left(W^{a} W^{b}\right)_{F}+$ h.c., where $W^{a}$ is a chiral Yang-Mills supermultiplet, the subscript denotes the F-component, and the matrix-valued function $F_{a b}(z, \bar{z})$ is not in general holomorphic. The remaining terms of dimension 8 and higher must be interpreted as arising from higher order spinorial derivatives of superfield operators.

As noted in I; the effective cut-off for effective theories derived from superstrings is field dependent [29], [44], [45]; moreover the field dependence is different for loop corrections arising from different sectors of the theory [29], [45]. As in I we use here a single cut-off and neglect its derivatives; terms involving derivatives of the cut-off have a different dependence on the moduli and must be considered together with terms that are one-loop finite. Our results, some of which are collected in the appendix, are presented in such a way that the contributions from different sectors can be isolated and the corresponding Pauli-Villars contributions can easily be evaluated.

This chapter only serves as a synopsis of the calculation of the one loop effective Lagrangian by giving a rather brief overview and outlining some general strategies and gauge fixing procedures. Also, in the subsequent sections of this chapter we establish our notations and conventions (which are somewhat different from the notations that we used in the introduction to the subject in Chapter 2.)

\subsection{Conventions}

Our space-time metric signature is $(+---)$. We use uppercase notation $(R, \Gamma)$ for derivatives of the Kähler metric, and lowercase $(r, \gamma)$ for those of the space-time metric. Our sign conventions for, respectively, the Riemann tensor, Ricci tensor and curvature scalar are as follows:

$$
\begin{gathered}
r_{\nu \rho \sigma}^{\mu}=g^{\mu \lambda} r_{\lambda \nu \rho \sigma}=\partial_{\sigma} \gamma_{\nu \rho}^{\mu}-\partial_{\rho} \gamma_{\nu \sigma}^{\mu}+\gamma_{\sigma \lambda}^{\mu} \gamma_{\nu \rho}^{\lambda}-\gamma_{\rho \lambda}^{\mu} \gamma_{\nu \sigma}^{\lambda} \\
r_{\mu \nu}=r_{\mu \rho \nu}^{\rho}, \quad r=g^{\mu \nu} r_{\mu \nu},
\end{gathered}
$$


and covariant differentiation is defined by

$$
\nabla_{\mu} A_{\nu}=\partial_{\mu} A_{\nu}-\gamma_{\mu \nu}^{\rho} A_{\rho}, \quad \text { etc. }
$$

The scalar field redefinition covariant quantities are defined identically with

$$
g_{\mu \nu} \rightarrow Z_{I J}, \quad \gamma \rightarrow \Gamma, \quad r \rightarrow R, \quad \nabla_{\mu} \rightarrow D_{I} \quad I=i, \bar{\imath}
$$

where $z^{i}, \bar{z}^{\bar{m}}=\left(z^{m}\right)^{\dagger}$ are the scalar partners of left and right handed Weyl fermions, respectively. Because the scalar metric is Kähler, there is only one type of nonvanishing element of the Riemann tensor, namely

$$
\begin{aligned}
& R_{j k \bar{m}}^{i}=\partial_{\bar{m}} \Gamma_{j k}^{i}=D_{\bar{m}} \Gamma_{j k}^{i}=-R_{j \bar{m} k}^{i}, \\
& R_{\bar{n} j k \bar{m}}=R_{\bar{n} k j \bar{m}}=R_{\bar{m} j k \bar{n}}=R_{\bar{m} k j \bar{n}} \\
& \quad=-R_{\bar{n} j \bar{m} k}=-R_{\bar{n} k \bar{m} j}=-R_{\bar{m} j \bar{n} k}=-R_{\bar{m} k \bar{n} j} .
\end{aligned}
$$

Note that since $R_{j k \ell}^{i}=0,\left[D_{i}, D_{j}\right]=0$, and the tensors

$$
A_{i_{1} \cdots i_{n}}=D_{i_{1}} \cdots D_{i_{n}} A, \quad \bar{A}^{i_{1} \cdots i_{n}}=D^{i_{1}} \cdots D^{i_{n}} \bar{A}
$$

are symmetric in all indices. It follows from the Bianchi identities that $D_{i} R_{j \bar{m} k}^{n}$ is totally symmetric in $\{i j k\}$.

We work in the Kähler covariant formalism [13], which differs from that of Cremmer et al. [42] by a phase transformation on the fermions that removes phases proportional to $\operatorname{Im}(W / \bar{W})$, where $W$ is the superpotential. In this formalism the fermion $\mathrm{U}(1) \mathrm{Kähler}$ connection is just

$$
\Gamma_{\mu}=\frac{i}{4}\left(K_{i} \mathcal{D}_{\mu} z^{i}-K_{\bar{m}} \mathcal{D}_{\mu} \bar{z}^{\bar{m}}\right)
$$

where $\mathcal{D}_{\mu}$ is the gauge covariant derivative. It is convenient to introduce the notation

$$
A=e^{K} W, \quad \bar{A}=e^{K} \bar{W} .
$$

Then the classical potential is $V=\hat{V}+\mathcal{D}$, where

$$
\hat{V}=e^{-K}\left(A_{i} \bar{A}^{i}-3 A \bar{A}\right), \quad \mathcal{D}=\frac{1}{2 x} \mathcal{D}_{a} \mathcal{D}^{a}, \quad \mathcal{D}_{a}=K_{i}\left(T^{a} z^{i}\right)
$$

With these conventions the tree level Lagrangian [42], [13] for the case $f(z)_{a b}=\delta_{a b} f(z)=$ $\delta_{a b}[x(z, \bar{z})+i y(z, \bar{z})]$ is 


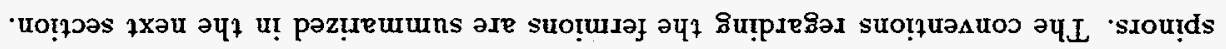

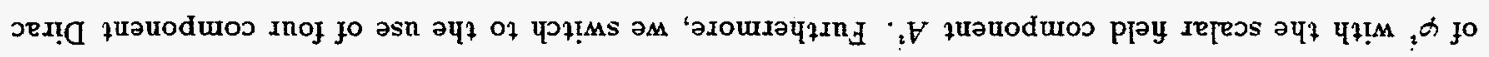

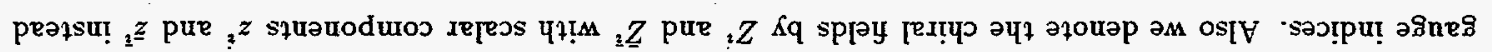

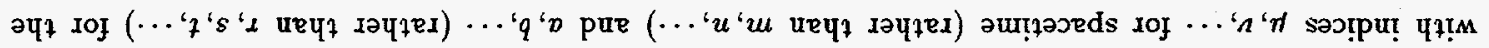

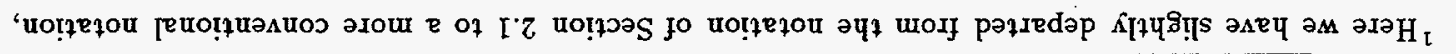

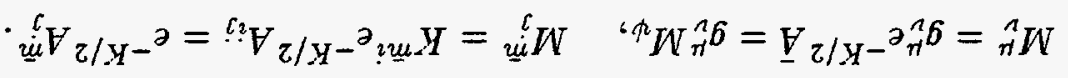

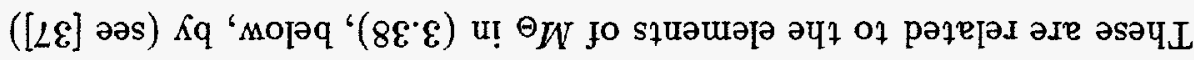

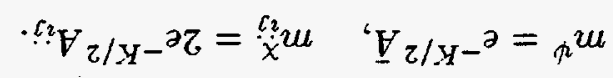

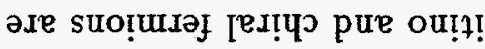

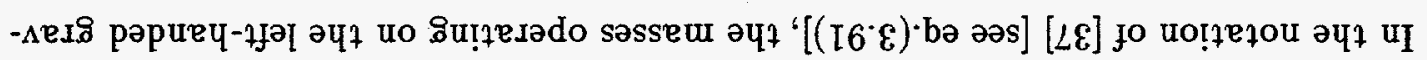

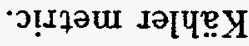

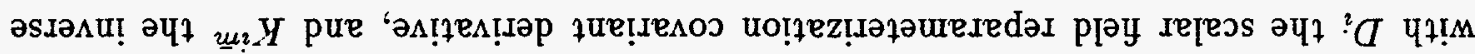

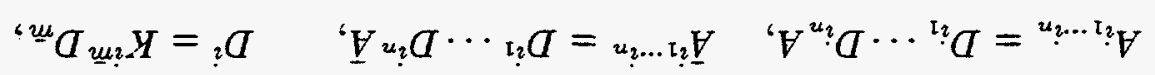

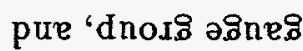

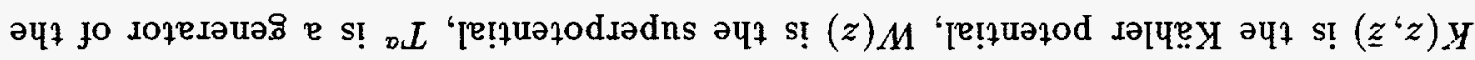

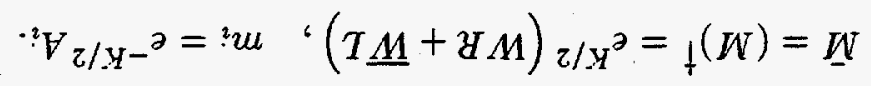

әдәчм

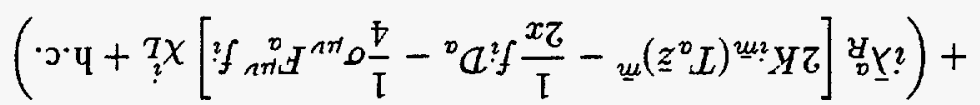

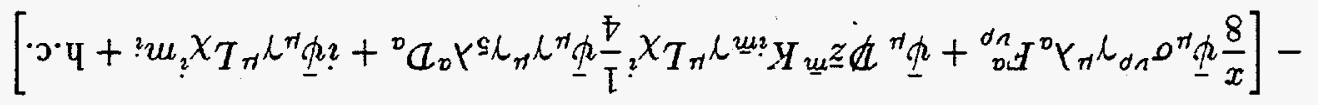

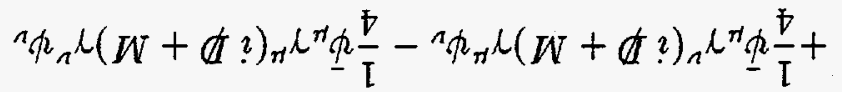

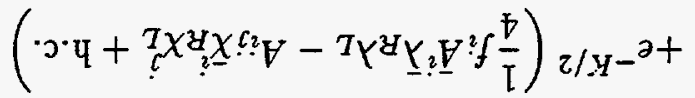

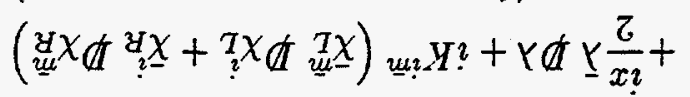

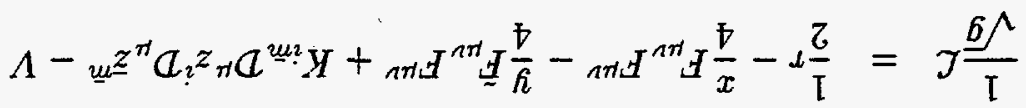

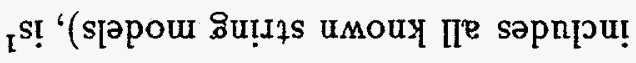

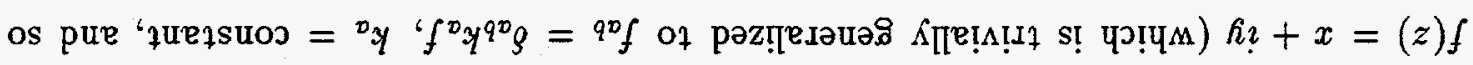

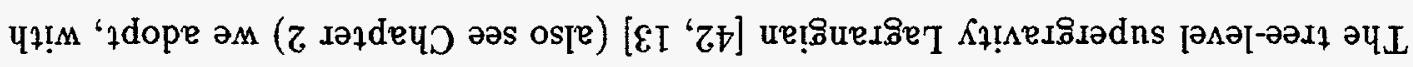


Note that the normalization of our chiral fermions is the same as in [42], which differs by a factor $\sqrt{2}$ from [13]. The covariant derivatives $D_{\mu}$ include the spin connection, the gauge connection, the Kähler connection (3.6), the affine connection, and the field reparameterization connection for chiral fields. For fermions:

$$
\begin{aligned}
\tilde{D}_{\mu} \psi & =\left[\nabla_{\mu}+\frac{1}{4} \gamma_{\nu}\left(\nabla_{\mu} \gamma^{\nu}\right)+i \gamma_{5} \Gamma_{\mu}\right] \psi \\
\tilde{D}_{\mu} \chi^{I} & =\left[\mathcal{D}_{\mu}+\frac{1}{4} \gamma_{\nu}\left(\nabla_{\mu} \gamma^{\nu}\right)-i \gamma_{5} \Gamma_{\mu}\right] \chi^{I}+\mathcal{D}_{\mu} z^{J} \Gamma_{J K}^{I} \chi^{K}
\end{aligned}
$$

[The gauginos have the same Kähler weight as the gravitino, and an additional connection (see below).] Operating on a function of scalar fields, $D_{\mu}=\mathcal{D}_{\mu} z^{I} D_{I}$, where $\mathcal{D}_{\mu}$ is gauge and general coordinate covariant.

\subsection{Dirac algebra}

We work in the Weyl representation for the Dirac matrices; for a flat metric:

$$
\begin{aligned}
& \gamma_{0}=\gamma^{0}=\left(\begin{array}{cc}
0 & -1 \\
-1 & 0
\end{array}\right), \quad \gamma^{i}=-\gamma_{i}=\left(\begin{array}{cc}
0 & \sigma^{i} \\
-\sigma^{i} & 0
\end{array}\right) \\
& \gamma_{5}=i \gamma^{0} \gamma^{1} \gamma^{2} \gamma^{3}=\left(\begin{array}{cc}
1 & 0 \\
0 & -1
\end{array}\right), \quad \sigma^{\mu \nu}=\frac{i}{2}\left[\gamma^{\mu}, \gamma^{\nu}\right] .
\end{aligned}
$$

To evaluate the fermion determinant, we note that an arbitrary $4 \times 4$ Dirac matrix $\mathcal{M}_{4}$ can be written as

$$
\mathcal{M}_{4}=R A R+L B L+R C L+L D R,
$$

where $A, B$ contain an even number of Dirac matrices $\gamma_{\nu}, C, D$ contain an odd number, $A, B, C, D$ have no explicit $\gamma_{5}$-dependence, and $L=\frac{1}{2}\left(1-\gamma_{5}\right)$ and $R=\frac{1}{2}\left(1+\gamma_{5}\right)$ are the helicity projection operators. Then $\operatorname{Tr} \mathcal{M}_{4}=\operatorname{Tr} R A+\operatorname{Tr} L B=\operatorname{Tr} \mathcal{M}_{8}$, where $\mathcal{M}_{8}$ is the $8 \times 8$ matrix

$$
\mathcal{M}_{8}=\left(\begin{array}{ll}
R A R & R C L \\
L D R & L B L
\end{array}\right),
$$

and $\operatorname{Tr} f\left(\mathcal{M}_{4}\right)=\operatorname{Tr} f\left(\mathcal{M}_{8}\right)$, where $f$ is any function that can be expanded in a Taylor series. Writing $\mathcal{M}_{4} \equiv \mathcal{M}_{4}\left(\gamma_{5}\right)$, we have

$$
\begin{aligned}
\mathcal{M}_{4}\left(-\gamma_{5}\right) & =R B R+L A L+R D L+L C R \\
\frac{1}{2}\left[\operatorname{Tr} \mathcal{M}_{4}\left(\gamma_{5}\right)+\operatorname{Tr} \mathcal{M}_{4}\left(-\gamma_{5}\right)\right] & =\frac{1}{2}(\operatorname{Tr} A+\operatorname{Tr} B)=\frac{1}{2} \operatorname{Tr}\left(\begin{array}{ll}
A & C \\
D & B
\end{array}\right) .
\end{aligned}
$$


Similarly, if $f$ is an arbitrary function of $\mathcal{M}_{4}$,

$$
\frac{1}{2}\left\{\operatorname{Tr} f\left[\mathcal{M}_{4}\left(\gamma_{5}\right)\right]+\operatorname{Tr} f\left[\mathcal{M}_{4}\left(-\gamma_{5}\right)\right]\right\}=\frac{1}{2} \operatorname{Tr} f(P), \quad P=\left(\begin{array}{ll}
A & C \\
D & B
\end{array}\right) .
$$

Setting $\mathcal{M}_{4}=-i \not D+M_{\Theta}, f\left(\mathcal{M}_{4}\right)=\ln \mathcal{M}_{4}$, (3.19) gives the trace $T_{+}$that has been evaluated previously ${ }^{2}[30,37,38,39,40]$. To evaluate the determinant $T_{-}$we define

$$
\mathcal{M}_{4}=\gamma_{0}\left(-i \not D+M_{\Theta}\right)
$$

which is a $4 \times 4$ matrix in Dirac space that we write [37] in terms of the $2 \times 2$ Pauli $\sigma$-matrices as

$$
\begin{aligned}
& \mathcal{M}_{4}=-\left(\begin{array}{cc}
i \tilde{\alpha} & C \\
\tilde{D} & i \beta
\end{array}\right), \quad \sigma_{ \pm}^{\mu}=(1, \pm \vec{\sigma}), \quad \sigma_{ \pm}^{\mu \nu}=\frac{i}{2}\left(\sigma_{ \pm}^{\mu} \sigma_{\mp}^{\nu}-\sigma_{ \pm}^{\nu} \sigma_{\mp}^{\mu}\right) \\
& \tilde{A}=\sigma_{+}^{\mu} d_{\mu}^{+}=\sigma_{+}^{\mu}\left[\tilde{D}_{\mu}^{+}-\tilde{L}_{\mu}\left(\sigma_{-}, \sigma_{+}\right)\right] \\
& B= \sigma_{-}^{\mu} d_{\mu}^{-}=\sigma_{-}^{\mu}\left[\tilde{D}_{\mu}^{-}-\tilde{L}_{\mu}\left(\sigma_{+}, \sigma_{-}\right)\right] \\
& C= m+M_{\mu \nu} \sigma_{+}^{\mu \nu} \equiv M\left(\sigma_{+}^{\mu \nu}\right), \quad \tilde{D}=\bar{m}+\bar{M}_{\mu \nu} \sigma_{-}^{\mu \nu} \equiv \bar{M}\left(\sigma_{-}^{\mu \nu}\right) \\
& \tilde{L}_{\mu}\left(\sigma_{-}, \sigma_{+}\right)=\frac{L_{\mu}}{24} \epsilon_{\lambda \nu \rho \sigma} \sigma_{-}^{\lambda} \sigma_{+}^{\nu} \sigma_{-}^{\rho} \sigma_{+}^{\sigma}
\end{aligned}
$$

The matrix elements in $\mathcal{M}_{4}$ are defined, up to the $\gamma_{5}$ ambiguity noted in [45], in terms of those appearing in the fermionic part of the quadratic quantum action (4.71) by:

$$
D_{\mu}=\tilde{D}_{\mu}+i \gamma_{5} L_{\mu}=i D_{\mu}^{+} R+i D_{\mu}^{-} L, \quad M_{\Theta}=\bar{M}\left(\sigma^{\mu \nu}\right) R+M\left(\sigma^{\mu \nu}\right) L
$$

The matrix-valued derivative operator $\tilde{D}_{\mu}$ is defined in (3.14) of I, the additional gaugino connection $L_{\mu}$ is given in (B.19) of the Appendix, and the elements of the mass matrix $M_{\Theta}=\bar{M} R+M L$ are given in (2.16), (2.17), (A.11) and (B.10) of I, together with (C.15) below. The tilde operation on $\mathbb{A}, \mathcal{B}, C, D$ amounts to the interchange $\sigma_{+} \leftrightarrow \sigma_{-}$. Thus

$$
\begin{aligned}
& \not L=\left(\begin{array}{cc}
0 & -\not L \\
0 & 0
\end{array}\right), \quad A R=\left(\begin{array}{cc}
0 & 0 \\
-\tilde{A} & 0
\end{array}\right), \\
& \not \tilde{A}=R\left(\tilde{D}^{+}-\frac{\not}{24} \epsilon_{\lambda \nu \rho \sigma} \gamma^{\lambda} \gamma^{\nu} \gamma^{\rho} \gamma^{\sigma}\right)^{2} R=R \not D^{2} R, \\
& \tilde{B} B=L\left(\tilde{D}^{-}-\frac{\not L}{24} \epsilon_{\lambda \nu \rho \sigma} \gamma^{\lambda} \gamma^{\nu} \gamma^{\rho} \gamma^{\sigma}\right)^{2} L=L \not D^{2} L,
\end{aligned}
$$

where the appropriate zero's in the transition from $2 \times 2$ to $4 \times 4$ matrices is implicit in the last two lines. More, generally, products of $\sigma_{ \pm}^{\mu}$ can be converted into products of $\gamma^{\mu}$

\footnotetext{
${ }^{2}$ The contributions from the terms $M_{\mu \nu} \sigma^{\mu \nu}$ were not fully included in [37].
} 
by

$$
\begin{aligned}
\left(\sigma_{+} \sigma_{-}\right)^{n} \sigma_{+} & \rightarrow-L \gamma^{2 n+1} R, \quad\left(\sigma_{-} \sigma_{+}\right)^{n} \sigma_{-} \rightarrow-R \gamma^{2 n+1} L, \\
\left(\sigma_{+} \sigma_{-}\right)^{n} & \rightarrow L \gamma^{2 n} L, \quad\left(\sigma_{-} \sigma_{+}\right)^{n} \rightarrow R \gamma^{2 n} R .
\end{aligned}
$$

Then defining

$$
\begin{aligned}
S_{ \pm} & =\frac{1}{2}\left[\operatorname{Tr} \ln \mathcal{M}_{4}(M, \vec{\sigma}) \pm \operatorname{Tr} \ln \mathcal{M}_{4}(-M,-\vec{\sigma})\right] \\
\mathcal{M}_{4}(-M,-\vec{\sigma}) & =-\left(\begin{array}{cc}
i & -\tilde{C} \\
-D & i \tilde{B}
\end{array}\right)=\mathcal{M}_{4}\left(-M,-\gamma_{5}\right) \gamma_{0}
\end{aligned}
$$

(3.23-24) immediately gives:

$$
\begin{aligned}
S_{+} & =\frac{1}{2} \operatorname{Tr} \ln \left[\mathcal{M}_{4}(-M,-\vec{\sigma}) \mathcal{M}_{4}(M, \vec{\sigma})\right] \\
& =\frac{1}{2} \operatorname{Tr} \ln \left(\begin{array}{cc}
-R\left[D_{+}^{2}+M \bar{M}\right] R & -R\left[i \not D^{+} M-M i \not D^{-}\right] L \\
-L\left[i \not D^{-} \bar{M}-\bar{M} i \not D^{+}\right] R & -L\left[\not D_{-}^{2}+\bar{M} M\right] L
\end{array}\right) \\
& =\frac{1}{2} \operatorname{Tr} \ln \left(-\not D^{2}-M_{\Theta}^{2}+i\left[\not D, M_{\Theta}\right]\right)=\frac{1}{2} \operatorname{Tr} \ln \left(-\hat{D}^{2}-H_{\Theta}^{2}\right) .
\end{aligned}
$$

where $\hat{D}=\hat{D}_{\Theta}$ and $H_{\Theta}$, defined in (4.31-32), are the operators that appear in the quantum Lagrangian, as we shall see shortly.. Although the matrix in (3.26) is $8 \times 8$, the helicity projection operators $L, R$ project out half the elements, so the counting of states is unchanged when we take the Dirac trace. Since $\operatorname{Tr} \ln \mathcal{M}(M)=\operatorname{Tr} \ln \mathcal{M}(-M)$, we have $S_{ \pm}=T_{ \pm}$, and (3.26) is equivalent to (3.19), up to the ambiguity described in [45]: terms even and odd in $\gamma_{5}$ can be interchanged using $\gamma_{5}=(i / 24) \epsilon^{\mu \nu \rho \sigma} \gamma_{\mu} \gamma_{\nu} \gamma_{\rho} \gamma_{\sigma}$.

The next step is to cast $S_{-}=T_{-}$in the form of (4.35), below, and to take its Fourier transform to obtain an expression of the form (4.36), but before performing the $p$-integration we write

$$
\begin{aligned}
\mathcal{M}^{-1} & {\left[\mathcal{M}\left(\gamma_{5}\right)-\mathcal{M}\left(-\gamma_{5}\right)\right]=\mathcal{M}^{-1} \mathcal{M}_{0}^{-1} \mathcal{M}_{0}\left[\mathcal{M}\left(\gamma_{5}\right)-\mathcal{M}\left(-\gamma_{5}\right)\right] } \\
& =2\left(D^{2}-\frac{i}{2} \sigma_{\mu \nu} G^{\mu \nu}+i D_{\mu} M^{\mu}\right)^{-1} i D_{\nu} N^{\nu}
\end{aligned}
$$

where $\mathcal{M}_{0}$ is ${ }^{3}$ the matrix (3.25) with

$$
C=D=0, \quad A_{\mu}=\tilde{D}_{\mu}^{+}+\tilde{L}_{\mu}\left(\sigma_{+}, \sigma_{-}\right), \quad B_{\mu}=\tilde{D}_{\mu}^{-}+\tilde{L}_{\mu}\left(\sigma_{-}, \sigma_{+}\right),
$$

\footnotetext{
${ }^{3}$ It might seem more efficient to take instead $\mathcal{M}_{0}=\mathcal{M}_{4}(-M,-\vec{\sigma})$ but this form turns out to introduce a spurious quadratic divergent term involving $M_{\mu \nu}$. To explicitly regulate ultraviolet (or infrared) divergences, one should introduce a regulator mass matrix $\mu_{0}$ and set $\mathcal{M}_{0} \rightarrow \mathcal{M}_{0}+\mu_{0}$; see the discussion in Section 4.3.
} 


$$
\begin{aligned}
& \frac{1}{2}\left[\mathcal{M}\left(\gamma_{5}\right)-\mathcal{M}\left(-\gamma_{5}\right)\right]=-\left(\begin{array}{cc}
\tilde{Y} & M_{I}\left(\sigma_{+}^{\mu \nu}\right) \\
-M_{I}\left(\sigma_{-}^{\mu \nu}\right) & -\searrow
\end{array}\right), \\
& \mathcal{J}_{\mu}=\frac{i}{2}\left(D_{\mu}^{+}-D_{\mu}^{-}\right), \quad M_{I}=\frac{1}{2}(M-\bar{M}),
\end{aligned}
$$

and

$$
N_{\mu}=\left(\begin{array}{cc}
-R \gamma_{\mu} \not{J} R & R \gamma_{\mu} M_{I} L \\
-L \gamma_{\mu} M_{I} R & L \gamma_{\mu} \not{J} L
\end{array}\right), \quad M_{\mu}=\left(\begin{array}{cc}
0 & R \gamma_{\mu} M L \\
L \gamma_{\mu} \bar{M} R & 0
\end{array}\right)
$$

We then redefine the integrand by [48]

$$
T(p, x) \rightarrow \dot{U} T(p, x) U^{-1}, \quad U=\exp \left(-i d \cdot \frac{\partial}{\partial p}\right) \exp \left(i \partial \cdot \frac{\partial}{\partial p}\right)
$$

which leaves the (properly regulated) integral unchanged. In the absence of background space-time curvature, the $8 \times 8$ matrix valued operator $d_{\mu}$ is simply

$$
d_{\mu}=D_{\mu}=\frac{\partial}{\partial x^{\mu}}+a_{\mu}(x)
$$

In the presence of space-time curvature, one has to expand [40] the action at $x^{\prime}=x+y$ in terms of normal coordinates, $\xi^{\mu}=y^{\mu}+\frac{1}{2} \gamma_{\rho \nu}^{\mu}(x) y^{\rho} y^{\nu}+O\left(\xi^{3}\right)$ :

$$
d_{\mu}=\frac{\partial}{\partial \xi^{\mu}}+a_{\mu}(x, \xi)
$$

where $\gamma_{\rho \nu}^{\mu}$ is the affine connection, and the full connection $a_{\mu}(x, \xi)$ includes terms that depend on the affine connection and its derivatives. The expansion of (3.27) for this case is determined in [40]. We then obtain:

$$
T=\int \frac{d^{4} p}{(2 \pi)^{4}} T(p, x)
$$

with

$$
\begin{aligned}
T(p, x) & =-\frac{1}{2} \operatorname{Tr} \ln \left[1+2 \Delta(x, p) p^{2} \mathcal{R}_{5}(x, p)\right] \\
\Delta^{-1} & =-T^{\mu \nu} \Delta_{\mu} \Delta_{\nu}+\hat{h}+X+\left(p^{\nu}+G^{\nu}\right) P_{\mu \nu} \widehat{M}^{\mu}, \\
\Delta_{\mu} & =p_{\mu}+G_{\mu}+\delta_{\mu}, \quad-p^{2} \mathcal{R}_{5}=\left(p^{\nu}+G^{\nu}\right) P_{\mu \nu} \hat{N}^{\mu}, \quad h=-\frac{i}{2} \sigma_{\mu \nu} G^{\mu \nu} \\
G_{\mu} & =\sum_{m=0} \frac{m+1}{(m+2) !}\left(-i D \cdot \frac{\partial}{\partial p}\right)^{m} G_{\nu \mu} \frac{\partial}{\partial p_{\nu}}, \quad G_{\mu \nu}=\left[D_{\mu}, D_{\nu}\right], \\
\hat{F} & =\sum_{0}^{\infty} \frac{(-i)^{n}}{n !}\left(D \cdot \frac{\partial}{\partial p}\right)^{n} F, \quad F=h, M^{\mu}, N^{\mu}, \quad D \cdot \frac{\partial}{\partial p} X \equiv\left[D_{\mu}, X\right] \frac{\partial}{\partial p_{\mu}}, \\
P^{\mu \nu} \gamma_{\nu} & =P^{\mu}=\gamma^{\mu}-\frac{1}{6} r^{\mu \rho \sigma \nu} \gamma_{\nu} \frac{\partial^{2}}{\partial p^{\rho} \partial p^{\sigma}}+O\left(\frac{\partial^{3}}{\partial p^{3}}\right),
\end{aligned}
$$




$$
\begin{aligned}
T^{\mu \nu} & =g^{\mu \nu}-\frac{1}{3} r^{\mu \rho \sigma \nu} \frac{\partial^{2}}{\partial p^{\rho} \partial p^{\sigma}}-\frac{i}{6} \nabla^{\lambda} r^{\mu \rho \sigma \nu} \frac{\partial^{3}}{\partial p^{\rho} \partial p^{\sigma} \partial p^{\lambda}}+O\left(\frac{\partial^{4}}{\partial p^{4}}\right) \\
X & =-\frac{r}{3}-\frac{i}{3} \nabla^{\mu} r \frac{\partial}{\partial p^{\mu}}+O\left(\frac{\partial^{2}}{\partial p}\right) \\
\delta_{\mu} & =\frac{i}{9}\left(\nabla_{\mu} r_{\rho \nu}-\nabla_{\nu} r_{\rho \mu}\right) \frac{\partial^{2}}{\partial p_{\nu} \partial p_{\rho}}+O\left(\frac{\partial^{3}}{\partial p^{3}}\right)
\end{aligned}
$$

Finally we write $\Delta^{-1}=-p^{2}(1+\mathcal{R})$ and expand

$$
\Delta=(1+\mathcal{R})^{-1}\left(-p^{-2}\right)=\sum_{n=0}(-\mathcal{R})^{n}\left(-p^{-2}\right)
$$

to obtain the expression (4.40), where we have set $\mu_{0}=0$.

Once all these manipulations have been performed we can simplify the expression for the fermion connection by using simply

$$
D_{\mu}^{ \pm}=\tilde{D}_{\mu}^{ \pm}+i \gamma_{5} L_{\mu}
$$

The point is that the part of the gaugino connection arising from the dilaton has been included in the "vector" $\left(\mathcal{J}_{\mu}^{V}\right)$ :

$$
\partial_{\mu}+\mathcal{J}_{\mu}^{V}=\frac{1}{2}\left(D_{\mu}^{+}+D_{\mu}^{-}\right)=\frac{1}{2}\left(\tilde{D}_{\mu}^{+}+\tilde{D}_{\mu}^{-}\right)+i \gamma_{5} L_{\mu}
$$

rather than in the "axial vector" $\left(\mathcal{J}_{\mu}\right)$ part of the connection.

We conclude this section by listing some Dirac traces that are useful in the evaluation of $T_{\text {- }}$ and of the ghostino and fermion determinants:

$$
\begin{gathered}
\operatorname{Tr} \gamma_{5} \gamma^{\mu} \gamma^{\nu} \gamma^{\rho} \gamma^{\sigma}=-4 i \epsilon^{\mu \nu \rho \sigma}, \quad \operatorname{Tr} \gamma_{5} \sigma^{\alpha \beta} \sigma^{\mu \nu}=4 i \epsilon^{\alpha \beta \mu \nu}, \quad \epsilon^{0123}=-g^{-1} \epsilon_{0123}=g^{-\frac{1}{2}}, \\
\operatorname{Tr}\left(\gamma_{5} \gamma^{\alpha} \gamma^{\beta} \gamma^{\gamma} \gamma^{\delta} \gamma^{\epsilon} \gamma^{\zeta}\right)=-4 i\left[\epsilon^{\gamma \delta \epsilon \zeta} g^{\alpha \beta}+\epsilon^{\alpha \beta \gamma \delta} g^{\epsilon \zeta}\right. \\
\left.+\epsilon^{\alpha \beta \epsilon \gamma} g^{\delta \zeta}+\epsilon^{\alpha \beta \gamma \zeta} g^{\delta \epsilon}+\epsilon^{\alpha \beta \delta \epsilon} g^{\gamma \zeta}+\epsilon^{\alpha \beta \zeta \delta} g^{\gamma \epsilon}+\epsilon^{\alpha \beta \epsilon \zeta} g^{\gamma \delta}\right], \\
\operatorname{Tr}\left(\gamma_{5} \sigma^{\alpha \beta} \gamma^{\gamma} \sigma^{\delta \epsilon} \gamma^{\zeta}\right)=4 i\left[\epsilon^{\alpha \beta \gamma \delta} g^{\epsilon \zeta}+\epsilon^{\alpha \beta \epsilon \gamma} g^{\delta \zeta}+\epsilon^{\alpha \beta \delta \epsilon} g^{\gamma \zeta}+\epsilon^{\alpha \beta \zeta \delta} g^{\gamma \epsilon}+\epsilon^{\alpha \beta \epsilon \zeta} g^{\gamma \delta}\right], \\
\operatorname{Tr}\left(\gamma_{5} \sigma^{\alpha \beta} \sigma^{\gamma \delta} \gamma^{\epsilon} \gamma^{\zeta}\right)=4 i\left[\epsilon^{\alpha \beta \gamma \delta} g^{\epsilon \zeta}+\epsilon^{\alpha \beta \epsilon \gamma} g^{\delta \zeta}+\epsilon^{\alpha \beta \gamma \zeta} g^{\delta \epsilon}+\epsilon^{\alpha \beta \delta \epsilon} g^{\gamma \zeta}+\epsilon^{\alpha \beta \zeta \delta} g^{\gamma \epsilon}\right], \\
\operatorname{Tr} \sigma_{\rho \sigma} \sigma^{\mu \nu} F_{a}^{\rho \sigma} F_{\mu \nu}^{b}=8 F_{a}^{\mu \nu} F_{\mu \nu}^{b}, \quad \operatorname{Tr} \sigma_{\rho \sigma} \sigma_{\mu \nu} \sigma_{\lambda \tau} F_{a}^{\rho \sigma} F_{b}^{\mu \nu} F_{c}^{\lambda \tau}=32 i F_{a}^{\mu \nu} F_{b \mu \rho} F_{c}^{\rho}, \\
\operatorname{Tr}(\sigma \cdot A \sigma \cdot B \sigma \cdot C \sigma \cdot D)=16\left[A^{\mu \nu} B^{\rho \sigma} C_{\mu \nu} D_{\rho \sigma}+(A \cdot B)(C \cdot D)+A^{\mu \nu}(B \cdot C) D_{\mu \nu}\right] \\
+64\left(A^{\mu \nu} B_{\mu \rho} C^{\rho \sigma} D_{\nu \sigma}-A^{\mu \nu} B_{\mu \rho} C_{\nu \sigma} D^{\rho \sigma}-A^{\mu \nu} B^{\rho \sigma} C_{\mu \rho} D_{\nu \sigma}\right),
\end{gathered}
$$




$$
\begin{aligned}
\operatorname{Tr}\left(\gamma^{\mu} \sigma \cdot A \gamma^{\nu} \sigma \cdot B\right) & =8\left(g^{\mu \nu} A_{\rho \sigma} B^{\rho \sigma}+2 A^{\mu \rho} B_{\rho}^{\nu}+2 A_{\rho}{ }^{\nu} B^{\mu \rho}\right) \\
\operatorname{Tr}\left(\gamma^{\mu} \gamma^{\nu} \sigma \cdot A \sigma \cdot B\right) & =8\left(g^{\mu \nu} A_{\rho \sigma} B^{\rho \sigma}+2 A^{\mu \rho} B_{\rho}^{\nu}-2 A_{\rho}{ }^{\nu} B^{\mu \rho}\right) \\
\operatorname{Tr}\left(Z_{\mu \nu} \gamma^{\mu} \sigma \cdot A \gamma^{\nu} \sigma \cdot B \gamma_{5}\right) & =8 i r_{\nu}^{\mu}\left(\tilde{A}^{\nu \rho} B_{\mu \rho}-A^{\nu \rho} \tilde{B}_{\mu \rho}\right)
\end{aligned}
$$

where $\sigma \cdot A=\sigma_{\mu \nu} A^{\mu \nu}$, etc., and $Z_{\mu \nu}=\frac{1}{4} \gamma^{\rho} \gamma^{\sigma} r_{\rho \sigma \nu \mu}$ is the field strength arising from the spin connection (note that $\gamma_{\mu} \gamma_{\nu} Z^{\mu \nu}=\frac{1}{2} r$ ). To evaluate the last trace in (3.37) we used the relations (A.14) and (B.25) of the Appendix.

\subsection{Quantum Lagrangian and Gauge Fixings (A Summary)}

The one-loop effective action is determined from the quadratic quantum action:

$$
\begin{aligned}
\mathcal{L}_{\text {quad }}(\Phi, \Theta, c)=\frac{1}{2} \hat{\phi}^{I} \hat{\phi}^{J}\left(\partial_{I} \partial_{J}+\left(A_{I}\right)_{J}^{K} \partial_{K}\right) S+\mathcal{L}_{g f}+\mathcal{L}_{g h}= \\
\quad-\frac{1}{2} \Phi^{T} Z^{\Phi}\left(\hat{D}_{\Phi}^{2}+H_{\Phi}\right) \Phi+\frac{1}{2} \bar{\Theta} Z^{\Theta}\left(i \not D_{\Theta}-M_{\Theta}\right) \Theta \\
+\frac{1}{2} \bar{c} Z^{c}\left(\hat{D}_{c}^{2}+H_{c}\right) c+O\left(\psi_{c l}\right),
\end{aligned}
$$

where $\phi^{I}=\Phi^{I}, \Theta^{I}, \partial_{I}=\partial / \partial \phi^{I}$, and the column vectors,

$$
\Phi^{T}=\left(h_{\mu \nu}, \hat{\mathcal{A}}^{a}, \hat{z}^{i}, \hat{z}^{\bar{m}}\right), \quad \Theta^{T}=\left(\psi_{\mu}, \lambda^{a}, \chi_{L}^{i}, \chi_{R}^{\bar{m}}, \alpha\right), \quad c^{T}=\left(c_{\nu}, c^{a}, c_{\alpha}\right),
$$

represent the boson, fermion and ghost quantum degrees of freedom, respectively, with $\alpha=-C \tilde{\alpha}^{T}$ an auxiliary field introduced [30] to implement gravitino gauge fixing. The connection $\left(A_{I}\right)_{J}^{K}$ in $(3.38)$, which is defined explicitly in $[30,31]$, is chosen so as to preserve all bosonic symmetries, and also to simplify matrix elements involving the graviton. In particular the quantum variables $\hat{z}^{i}, \hat{z}^{\bar{m}}$ are normal coordinates in the space of scalar fields: $\left(A_{i}\right)_{j}^{k}=\Gamma_{i j}^{k}$ is the affine connection associated with the Kähler metric $K_{i \bar{m}}$, giving a scalar field reparameterization invariant expansion. In (3.38) $\psi_{c l}$ represents background fermion fields that we set to zero; that is, we calculate only the one-loop bosonic action.

For the boson sector, we use a smeared gauge-fixing:

$$
\begin{aligned}
& \mathcal{L} \rightarrow \mathcal{L}+\mathcal{L}_{g f}, \quad \mathcal{L}_{g f}=-\frac{\sqrt{g}}{2} C_{A} Z^{A B} C_{B}, \\
& Z=\left(\begin{array}{cc}
\delta^{a b} & 0 \\
0 & -g^{\mu \nu}
\end{array}\right), \quad C=\left(\begin{array}{c}
C_{a} \\
C_{\mu}
\end{array}\right) .
\end{aligned}
$$

The Yang-Mills gauge-fixing term:

$$
C^{a}=\mathcal{D}^{\prime \mu} \hat{\mathcal{A}}_{\mu}^{a}+\frac{i}{\sqrt{x}} K_{i \bar{m}}\left[\left(T^{a}\right)^{\bar{m}} \hat{z}^{i}-\left(T^{a}\right)^{i} \hat{z}^{\bar{m}}\right]
$$


preserves off-shell supersymmetry [33] in the limit of global supersymmetry and coincides with the string-loop result [34] for chiral multiplet wave function renormalization. The graviton gauge-fixing term:

$$
\sqrt{2} C_{\mu}=\left(\nabla^{\nu} h_{\mu \nu}-\frac{1}{2} \nabla_{\mu} h_{\nu}^{\nu}-2 \mathcal{D}_{\mu} z^{I} Z_{I J} \hat{z}^{J}+2 \mathcal{F}_{\mu \nu}^{a} \hat{\mathcal{A}}_{a}^{\nu}\right)
$$

is the one originally introduced by 't Hooft and Veltman [35], generalized [30] to include the Yang-Mills sector. The script quantum and classical Yang-Mills fields and field strengths are canonically normalized [41]:

$$
\mathcal{A}_{\mu}=\sqrt{x} A_{\mu}, \quad \hat{\mathcal{A}}_{\mu}=\sqrt{x} \hat{A}_{\mu}, \quad \mathcal{F}_{\mu \nu}=\sqrt{x} F_{\mu \nu}, \sqrt{x} \mathcal{D}_{\mu} A_{\nu}=\mathcal{D}_{\mu}^{\prime} \mathcal{A}_{\mu}
$$

where $\mathcal{D}_{\mu}$ is the gauge and general covariant derivative, and $\mathcal{D}_{\mu}^{\prime}=\mathcal{D}_{\mu}-\partial_{\mu} x / 2 x, \mathcal{D}_{\mu}^{\prime \prime}=$ $\mathcal{D}_{\mu}+\partial_{\mu} x / 2 x$. In the earlier literature two gravitino gauge fixing procedures have been used: a) a Landau-type gauge $[36,37] \gamma \cdot \psi=0$, implemented by the introduction of an auxiliary field, and b) a smeared gauge [39] $\mathcal{L} \rightarrow \mathcal{L}-\bar{F} \mathcal{M} F, F=\gamma \cdot \psi, \mathcal{M}=$ $\frac{1}{4}\left(i \not D+2 M_{\psi}\right)$ supplemented with Nielsen-Kallosh ghosts. Here we use an unsmeared gauge $G=0$, with the gauge-fixing function [30]

$$
\begin{aligned}
G= & -\gamma^{\nu}(i \not D-\bar{M}) \psi_{\nu}-2\left(\not \supset z^{i} K_{i \bar{m}} R \chi^{\bar{m}}+\not D \bar{z}^{\bar{m}} K_{i \bar{m}} L \chi^{i}\right) \\
& +\frac{x}{2} \sigma^{\nu \rho} \lambda_{a} F_{\nu \rho}^{a}+2 i m_{I} \chi^{I}-\gamma_{5} \mathcal{D}_{a} \lambda^{a}
\end{aligned}
$$

where $D_{\mu}$ contains the spin and chiral Kähler connections. The quantum Lagrangian is obtained by the introduction of an auxiliary field $\alpha: \delta(G)=\int d \alpha \exp (i \alpha G)$, and a shift in the gravitino field: $\psi^{\prime}=\psi+\gamma \alpha, \bar{\psi}^{\prime}=\bar{\psi}+\bar{\alpha} \gamma$, so as to diagonalize the gravitino kinetic energy term. The ghost and ghostino determinants are obtained in the usual way as, respectively:

$$
\left(\hat{D}_{c}^{2}+H_{c}\right)_{B}^{A}=\frac{\partial}{\partial \epsilon_{A}} \delta C_{B}, \quad A, B=a, \mu, \quad\left(\hat{D}_{c}^{2}+H_{c}\right)_{\beta}^{\alpha}=\frac{\partial \delta G^{\alpha}}{\partial \epsilon^{\beta}},
$$

where $\hat{D}_{\mu}$ is related to $\mathcal{D}_{\mu}$ or $D_{\mu}$ by additional connections. With these choices the one-loop bosonic action takes a very simple form:

$$
\begin{aligned}
S_{1}= & \frac{i}{2} \operatorname{Tr} \ln \left(\hat{D}_{\Phi}^{2}+H_{\Phi}\right)-\frac{i}{2} \operatorname{Tr} \ln \left(-i \not D_{\Theta}+M_{\Theta}\right) \\
& +\frac{i}{2} S \operatorname{Tr} \ln \left(\hat{D}_{c}^{2}+H_{c}\right),
\end{aligned}
$$

where

$$
\mathrm{STr} \ln \left(\hat{D}_{c}^{2}+H_{c}\right)=2 \operatorname{Tr} \ln \left(\hat{D}_{c}^{2}+H_{c}\right)_{c_{\alpha}}-2 \operatorname{Tr} \ln \left(\hat{D}_{c}^{2}+H_{c}\right)_{c_{a, \mu}}
$$


which just reduces to determinants of the form of those for scalars and spin- $\frac{1}{2}$ fermions. Moreover the ghost and non-ghost sectors have separately supersymmetric quantum spectra, except for the Yang-Mills fields:

$$
\frac{1}{2}(\operatorname{Tr} 1)_{\Theta}=(\operatorname{Tr} 1)_{\Phi}-2 N_{G}=2 N+2 N_{G}+10 . \quad(\operatorname{Tr} 1)_{c_{\alpha}}=4, \quad(\operatorname{Tr} 1)_{c_{a, b}}=4+N_{G}
$$

where $N\left(N_{G}\right)$ is the number of chiral (gauge) supermultiplets. To evaluate (3.41) we separate $[37,31]$ the fermion determinant into helicity-even and -odd parts:

$$
-\frac{i}{2} \operatorname{Tr} \ln \left(-i \not D+M_{\Theta}\right) \equiv-\frac{i}{2} \operatorname{Tr} \ln \mathcal{M}\left(\gamma_{5}\right)=T_{-}+T_{+}
$$

where here $D_{\mu}$ contains all fermion connections, and

$$
\begin{aligned}
& T_{-}=-\frac{i}{4}\left[\operatorname{Tr} \ln \mathcal{M}\left(\gamma_{5}\right)-\operatorname{Tr} \ln \mathcal{M}\left(-\gamma_{5}\right)\right], \\
& T_{+}=-\frac{i}{4}\left[\operatorname{Tr} \ln \mathcal{M}\left(\gamma_{5}\right)+\operatorname{Tr} \ln \mathcal{M}\left(-\gamma_{5}\right)\right] \\
& \mathcal{M}=\gamma_{0}\left(-i \not D+M_{\Theta}\right)=\left(\begin{array}{cc}
\sigma_{+}^{\mu} D_{\mu}^{+} & M^{+} \\
M^{-} & \sigma_{-}^{\mu} D_{\mu}^{-}
\end{array}\right), \quad \sigma_{ \pm}^{\mu}=(1, \pm \vec{\sigma}) .
\end{aligned}
$$

Then defining

$$
\hat{D}_{\Theta}^{2}+H_{\Theta} \equiv\left(-i \not D_{\Theta}+M_{\Theta}\right)\left(i \not D_{\Theta}+M_{\Theta}\right)
$$

The one-loop bosonic action (3.41) reduces to:

$$
S_{1}=\frac{i}{2} \mathrm{~S} \operatorname{Tr} \ln \left(\hat{D}^{2}+H\right)+T_{-} .
$$

The helicity-odd term $T_{-}$is at most logarithmically divergent, and is finite $[30,31]$ in the absence of a dilaton, that is, for $f(Z)=g^{-2}+i \theta / 8 \pi^{2}=$ constant. As discussed in $[45,31]$ there is an ambiguity in the separation (3:43) of the fermion determinant into helicity-even and -odd contributions, because terms that are even and odd in $\gamma_{5}$ can be interchanged by the use of the identities:

$$
\gamma_{5}=(i / 24) \epsilon^{\mu \nu \rho \sigma} \gamma_{\mu} \gamma_{\nu} \gamma_{\rho} \gamma_{\sigma}, \quad \sigma_{\mu \nu}=i \gamma_{5} \sigma^{\rho \sigma} \epsilon_{\rho \sigma \mu \nu}, \quad \text { etc. }
$$

In most cases the choice is dictated by gauge or Kähler covariance. However supersymmetry must be used to fix the off-diagonal gaugino- $\alpha$ and gangino-dilaton mass terms:

$$
\begin{aligned}
M_{\alpha \lambda^{a}} & =-\sqrt{\frac{x}{2}} F_{a}^{\mu \nu} \sigma_{\mu \nu} \\
M_{\chi^{i} \lambda^{a}} & =-i \frac{f_{i}}{4 \sqrt{x}}\left(F_{a}^{\mu \nu}-i \gamma_{5} \tilde{F}_{a}^{\mu \nu}\right) \sigma_{\mu \nu}
\end{aligned}
$$


and the dilaton-dependent gaugino connection:

$$
A_{\lambda^{a} \lambda^{b}}^{\mu}=\delta_{a b} \frac{\partial^{\mu} y}{2 x} \frac{\epsilon^{\lambda \nu \rho \sigma}}{24} \gamma_{\lambda} \gamma_{\nu} \gamma_{\rho} \gamma_{\sigma}
$$

(3.45) and (3.46) are precisely the choices that allow Pauli-Villars regularization of the quadratic divergences [45]. The choice (3.46) further insures the nonrenormalization [58] of the topological charge $\theta=8 \pi^{2} y$, and is consistent with linear-chiral multiplet duality $[60]$ for the dilaton supermultiplet.

The above summarizes our conventions and choices of gauge. We present further details of these gauge fixings and the calculation of the one-loop corrected effective Lagrangian of supergravity coupled to SYM and matter in the following chapter. 


\section{Chapter 4}

\section{The One Loop Calculation}

\subsection{Introduction}

In this chapter we present the calculation of the one-loop effective Lagrangian for $N=1$ supergravity, Yang-Mills, and chiral matter system. In Section 2 we discuss gauge fixing and the definition of the action expansion in more detail than outlined in Chapter 3, and in Section 3 we evaluate the helicity-odd fermion loop contributions. Our result for the one-loop corrected effective action is given in Section 4, and applied to generic models from string theory in Section 5 . We summarize our results and discuss applications in Section 6.

In Appendix A we specify our Yang-Mills sign conventions and list relations among the covariant scalar derivatives of the Kähler potential $K$, the superpotential $W$ and the gauge field normalization function $f$ that follow from gauge invariance of these functions and that are useful in evaluating traces. Appendix B contains the matrix elements of the operators that appear in the one-loop effective action and the traces that needed to evaluate the divergent contributions [equations (4.72-75) below].

\subsection{Gauge Fixing and the Expansion of the Action}

The S-matrix is independent of gauge fixing and also of shifts in the propagators that are proportional to $\mathcal{L}_{A}=\partial \mathcal{L} / \partial \phi^{A}$ where $\phi^{A}$ is any field. However, certain choices can lead to an effective Lagrangian that better displays the symmetries of the theory. For example, we expand the action $S$ in terms of normal scalar coordinates $[47,48] \hat{z}^{I}$ :

$$
S=S(z)+\left.D_{I} S\right|_{z} \hat{z}^{I}+\left.\frac{1}{2} D_{I} D_{J} S\right|_{z} \hat{z}^{I} \hat{z}^{J}+\cdots
$$

where $D_{I}$ is the field redefinition covariant derivative and interpret the determinant of the second term in (4.1) as the one-loop effective action for a scalar theory. This is different from that of a standard Taylor expansion by terms of the form $F^{J L}(z) \Gamma(z)_{J K}^{I}\left(D_{I} S\right)_{z}$, where $\Gamma_{J K}^{I}$ is the connection associated with the covariant derivative $D_{I}$, and $F^{J L}$ is an arbitrary matrix-valued function of the background scalar fields. Such terms vanish by 
the classical equations of motion for the background fields $z:\left.D_{I} S\right|_{z}=\partial_{I} S \mid z=0$. The expansion (4.1) yields a manifestly field redefinition invariant effective action. It therefore preserves nonlinear symmetries among the scalar fields, up to quantum anomalies.

Supersymmetry is also a nonlinear symmetry in supergravity theories, even when auxiliary fields are used. We have no formal argument by which we can determine the gauge fixing and expansion prescription so as to yield an effective action that is manifestly supersymmetric. ${ }^{1}$ Instead, we adopt a pragmatic approach, and use prescriptions that give the most boson-fermion cancellations, and/or simplify the calculation. We find that with our prescription the operators of dimension six or less can be interpreted as renormalizations of the tree Lagrangian, except for those that depend on the scalar curvature tensor. Additional operators of dimension eight can be isolated into terms of the form $\left.F^{J L} \Gamma_{J K}^{I} D_{I} S\right|_{z}$, which do not contribute to the S-matrix. It turns out that the gauge fixing prescription with these properties yields an effective quantum Lagrangian that is of a particularly simple form: all the propagators are the same as those of standard scalar or spin- $\frac{1}{2}$ fermions. It is possible that this feature contributes to the enhanced cancellations.

\subsubsection{Gauge-fixing the gravity supermultiplet}

We set background fermions to zero, and use unhatted symbols for quantum fermion fields $(\psi, \chi, \lambda)$. The commonly used gauge fixing for the graviton $[35,50,37,39]$, when generalized to include the YM sector, is defined by

$$
\begin{gathered}
\mathcal{L} \rightarrow \mathcal{L}+\frac{1}{2} C_{\mu} C^{\mu}, \\
C_{\mu}=\frac{1}{\sqrt{2}}\left(\nabla^{\nu} h_{\mu \nu}-\frac{1}{2} \nabla_{\mu} h_{\nu}^{\nu}-2 \mathcal{D}_{\mu} z^{I} Z_{I J} \hat{z}^{J}+2 x F_{\mu \nu}^{a} \hat{A}_{a}^{\nu}\right),
\end{gathered}
$$

where $Z_{I J}(z, \bar{z})$ is the scalar metric, $\hat{z}, \hat{A}$ are the quantum scalar and gauge fields, and the symmetric tensor $h_{\mu \nu}$ is the quantum part of the gravitational field. The gauge fixing (4.2) leads to a Lagrangian of the desired form, (3.38).

For the gravitino, two types of gauge fixing have been used: the Landau gauge $[36,37]$ $\gamma \cdot \psi=0$, which is implemented with the aid of an auxiliary field, and the smeared gauge

\footnotetext{
${ }^{1}$ Since we set background fermions to zero, our effective action cannot be manifestly supersymmetric. However supersymmetry constrains $[42,13]$ the bosonic part of the action; by "manifest supersymmetry" we are referring to these constraints.
} 
fixing [39] $\mathcal{L} \rightarrow \mathcal{L}-\bar{F} \mathcal{M} F, F=\gamma \cdot \psi, \mathcal{M}=\frac{1}{4}\left(i \not D+2 M_{\psi}\right)$, which requires NielsenKallosh ghosts. Neither of these has the feature that the quantum Lagrangian reduces to the simple form (3.38). In addition, while the Landau gauge propagators have the correct poles for constant background fields, the smeared gauge fixing propagators do not. Here we adopt an unsmeared gauge which satisfies both requirements.

In a supergravity theory in which the Yang-Mills normalization function satisfies $\operatorname{Re} f_{a b}=\delta_{a b} x$, the part of the Lagrangian that depends on the gravitino $\psi_{\mu}$ is $[42,13]$

$$
\begin{aligned}
\mathcal{L}_{\psi}= & \frac{1}{4} \bar{\psi}_{\mu} \gamma^{\nu}(i \not D+M) \gamma^{\mu} \psi_{\nu}-\frac{1}{4} \bar{\psi}_{\mu} \gamma^{\mu}(i \not D+M) \gamma^{\nu} \psi_{\nu}+\left[\frac{x}{8} \bar{\psi}_{\mu} \sigma^{\nu \rho} \gamma^{\mu} \lambda_{a} F_{\nu \rho}^{a}\right. \\
& \left.-\bar{\psi}_{\mu} \not \bar{z}^{\bar{m}} K_{i \bar{m}} \gamma^{\mu} L \chi^{i}+\frac{1}{4} \bar{\psi}_{\mu} \gamma^{\mu} \gamma_{5} \lambda^{a} \mathcal{D}_{a}-i \bar{\psi}_{\mu} \gamma^{\mu} L \chi^{i} m_{i}+\text { h.c. }\right] \\
& \text { +four - fermion terms. }
\end{aligned}
$$

where

$$
\begin{array}{cc}
\vec{M}=(M)^{\dagger}=e^{K / 2}(W R+\bar{W} L), & R, L=\frac{1}{2}\left(1 \pm \gamma_{5}\right) \\
m_{n}=\left(\bar{m}_{\bar{n}}\right)^{\dagger}=e^{-K / 2} D_{i}\left(e^{K} W\right), & \mathcal{D}_{a}=K_{i}\left(T_{a} z\right)^{i}
\end{array}
$$

We take the Landau gauge condition $G=0$, where

$$
\begin{aligned}
G= & -\gamma^{\nu}(i \not D-\bar{M}) \psi_{\nu}-\frac{x}{2} \sigma^{\nu \rho} \lambda_{a} F_{\nu \rho}^{a} \\
& -2\left(\not D z^{i} K_{i \bar{m}} R \chi^{\bar{m}}+\not \supset \bar{z}^{\bar{m}} K_{i \bar{m}} L \chi^{i}\right)+2 i m_{I} \chi^{I}-\gamma_{5} \mathcal{D}_{a} \lambda^{a}
\end{aligned}
$$

which we implement by inserting a $\delta$-function in the functional integral over $\hat{\phi}$. Writing

$$
\delta[G]=\int d \alpha \exp (i \alpha G)
$$

and defining

$$
\psi^{\prime}=\psi+\gamma \alpha, \quad \bar{\psi}^{\prime}=\bar{\psi}+\bar{\alpha} \gamma
$$

We obtain

$$
\begin{aligned}
\mathcal{L}= & -\frac{1}{2} \bar{\psi}^{\prime \mu}(i \not D-\bar{M}) \psi_{\mu}^{\prime}+\frac{1}{2} \bar{\alpha} \gamma^{\mu}(i \not D-\bar{M}) \gamma_{\mu} \alpha+\text { matter terms } \\
= & -\frac{1}{2} \bar{\psi}^{\prime \mu}(i \not D-\bar{M}) \psi_{\mu}^{\prime}-\bar{\alpha}(i \not D+2 M) \alpha+\bar{\alpha}\left(\frac{x}{2} \sigma^{\nu \rho} \lambda_{a} F_{\nu \rho}^{a}+2 i m_{I} \chi^{I}-\gamma_{5} \mathcal{D}_{a} \lambda^{a}\right) \\
& -i x \bar{\psi}_{\mu}^{\prime}{F_{a}^{\mu}}_{a}^{a}-2 \bar{\psi}_{\mu}^{\prime}\left(\mathcal{D}^{\mu} \bar{z}^{\bar{m}} K_{i \bar{m}} L \chi^{i}+\mathcal{D}^{\mu} z^{i} K_{i \bar{m}} R \chi^{\bar{m}}\right) .
\end{aligned}
$$

Note that $\psi$ is C-even: $\psi=C \bar{\psi}^{T}$, then $\psi^{\prime}=C \bar{\psi}^{T}$ requires $\alpha=-C \bar{\alpha}^{T}$, i.e. $\alpha$ is C-odd; 
note also that $\alpha$ has negative metric. ${ }^{2}$ All the terms remaining in the Lagrangian (4.6) are of the form of either a mass or a connection; that is, (4.6) is of the form (3.38).

To obtain the ghostino determinant we use the supersymmetry transformations [42]

$$
\begin{gathered}
i \delta \psi_{\mu}=\left(i D_{\mu}-\frac{1}{2} \gamma_{\mu} M\right) \epsilon, \quad i \delta \chi^{i}=\frac{1}{2}\left(\not \mathcal{D} z^{i} R-i \bar{m}^{i} L\right) \epsilon \\
i \delta \chi^{\bar{m}}=\left[\frac{1}{2}\left(\not \mathcal{D} \bar{z}^{\bar{m}} L-i m^{\bar{m}} R\right)\right] \epsilon, \quad \bar{m}^{i}=K^{i \bar{m}} \bar{m}_{\bar{m}}, \quad m^{\bar{m}}=K^{i \bar{m}} m_{i} \\
i \delta \lambda^{a}=\left[-\frac{i}{4} \gamma^{\mu} \gamma^{\nu} F_{\mu \nu}^{a}-\frac{1}{2 x} \mathcal{D}^{a}\right] \epsilon
\end{gathered}
$$

to obtain

$$
\begin{aligned}
\frac{\partial \delta G}{\partial \epsilon}= & D^{\mu} D_{\mu}-\frac{1}{2} \gamma^{\mu} \gamma^{\nu}\left[D_{\mu}, D_{\nu}\right]-i[\not D, M]-2 M \bar{M}+\bar{m}^{i} m_{i}+\mathcal{D} \\
& +2 i \bar{m}_{\bar{m}} \not \bar{z}^{\bar{m}} L+2 i m_{i} \not D z^{i} R+\frac{x}{2} \sigma_{\sigma \rho} F_{a}^{\sigma \rho}\left[\frac{1}{4} \sigma^{\mu \nu} F_{\mu \nu}^{a}+\frac{1}{x} \gamma_{5} \mathcal{D}^{a}\right] \\
& -\mathcal{D}_{\mu} z^{i} K_{i \bar{m}} \mathcal{D}^{\mu} \bar{z}^{\bar{m}}+\frac{1}{2} \gamma_{5}\left[\gamma^{\mu}, \gamma^{\nu}\right] \mathcal{D}_{\mu} \bar{z}^{\bar{m}} K_{i \bar{m}} \mathcal{D}_{\nu} z^{i}
\end{aligned}
$$

For constant background fields the ghostino propagator becomes

$$
D^{\mu} D_{\mu}-2 M \bar{M}+\bar{m}^{i} m_{i}+\mathcal{D}=D^{\mu} D_{\mu}+M \bar{M}+V
$$

where $V$ is the potential. When we evaluate this at a ground state with a flat background metric, the vacuum energy necessarily vanishes: $V=0$, so the (4-fold) ghostino pole is at $p^{2}=-D^{2}=M^{2}$ which is the correct pole for unitarity. If the cosmological constant is nonzero the curvature is also, and there are additional terms in all the masses.

\subsubsection{Yang-Mills Gauge Fixing}

We first discuss the simpler case of flat SUSY Yang-Mills, where a similar gauge fixing dependence arises [33], and where a "supersymmetric gauge" can be found.

In background field calculations of the effective one-loop action, the Landau gauge fixing condition $\mathcal{D}^{\mu} \hat{A}_{\mu}=0$ has frequently been used $[37,41,40]$. For $W=0$ (i.e., no superpotential), the dimension four operators of the resulting supergravity Lagrangian for the gauge nonsinglet scalars can be interpreted in terms of two renormalizations:

\footnotetext{
${ }^{2}$ In the notation of (3.1), $Z_{\alpha \alpha}=-2$; including the contribution proportional to $\operatorname{Det} Z_{\alpha \alpha}$ we get a quartically divergent term proportional to $\ln 2$ which cancels a similar contribution from the graviton ghost [37].
} 
the renormalization of the gauge kinetic function, $x_{b}^{a}(z, \bar{z})=\operatorname{Re} f(z)_{b}^{a}$, and that of the Kähler potential $K(z, \bar{z})$. Here (and throughout) we consider the case $x_{b}^{a}=\delta_{b}^{a} x$ at tree level, for which the results are:

$$
\delta K=\frac{\ln \Lambda^{2}}{32 \pi^{2}}\left[-\frac{2}{x} K_{\bar{m} j}\left(T_{a} \bar{z}\right)^{\bar{m}}\left(T^{a} z\right)^{j}\right]+\text { higher dimension terms }
$$

where $T^{a}$ represents the gauge group on the scalar field $z^{n}=\left(\bar{z}^{\bar{n}}\right)^{\dagger}$, and

$$
\delta x_{a}^{b}=\frac{\ln \Lambda^{2}}{32 \pi^{2}}\left[2 D_{i}\left(T_{a} z\right)^{j} D_{j}\left(T^{b} z\right)^{i}-6 C_{G}^{(a)} \delta_{a}^{b}\right]+\text { higher dimension terms }
$$

where $C_{G}^{(\alpha)}$ is the Casimir of the adjoint representation and the field redefinition covariant scalar derivative $D_{i}$ is defined in Chapter 3. The fact that (4.11) is not the real part of a holomorphic function has been discussed elsewhere in the literature (see, e.g., [26]). In the flat SUSY limit $x \rightarrow$ constant, $K_{i \bar{m}} \rightarrow \delta_{i m}$, and the renormalizations reduce to constants that depend on the Casimirs of the matter representations $R$ :

$$
\begin{gathered}
\delta K_{i \bar{m}} \rightarrow-\frac{\ln \Lambda^{2}}{16 \pi^{2} x} \sum_{a}\left(T_{a}\right)_{i \bar{m}}^{2}=-\delta_{i m} \frac{\ln \Lambda^{2}}{16 \pi^{2} x} \sum_{a} C_{2}^{a}\left(R_{i}\right), \\
\delta x_{a}^{b}=\delta_{b}^{a} \frac{\ln \Lambda^{2}}{16 \pi^{2} x} \operatorname{Tr}\left(T_{a}\right)^{2}=\delta_{b}^{a} \frac{\ln \Lambda^{2}}{16 \pi^{2} x} \sum_{R} C_{R}^{a} .
\end{gathered}
$$

When a superpotential is included, the results obtained in the Landau gauge can no longer be interpreted in terms of these renormalizations. This is similar to the result found in [33]. However, if we use a smeared gauge fixing prescription defined by

$$
\mathcal{L} \rightarrow \mathcal{L}-\frac{x}{2} C_{a} C^{a}, \quad C^{a}=\mathcal{D}^{\mu} \hat{A}_{\mu}^{a}+\frac{i}{x}\left[\left(T^{a} \bar{z}\right)^{\bar{m}} \hat{z}^{i}-\left(T^{a} \bar{z}\right)^{i} \hat{z}^{\bar{m}}\right] K_{i \bar{m}},
$$

the results can once again be interpreted as above, with, instead of (4.10),

$$
\delta K=\frac{\ln \Lambda^{2}}{32 \pi^{2}}\left(-\frac{4}{x} K_{\bar{m} j}\left(\mathcal{T}_{a} \bar{z}\right)^{\bar{m}}\left(T^{a} z\right)^{j}+e^{-K} A_{i j} \bar{A}^{i j}\right)+\text { higher dimension terms }
$$

where $A_{i j}$ is defined in Section 3.2 ; in the flat SUSY limit it reduces to the second derivative of the superpotential $W$ :

$$
e^{-K} A_{i j} \bar{A}^{i j} \rightarrow e^{K} W_{i j} \bar{W}^{i j}
$$

Note that the gauge-dependent term in (4.13) differs by a factor of two from that in (4.10). The result (4.13) agrees with the chiral matter wave function renormalization found in [33] and in a recent string loop calculation [34]. 
Unlike the Landau gauge, the smeared gauge fixing (4.12) gives a quantum Lagrangian of the simple quadratic form. The field-dependent masses as we have seen have the correct poles for unitarity when evaluated at the ground state configuration for the background fields, i.e., $\mathcal{D}_{\mu} z=A_{\mu}=\partial_{i} V=0$, where $V$ is the scalar potential [30]. We will use gauge fixing prescriptions for supergravity that share this feature.

In the general supergravity Lagrangian [42], the function $f_{a b}(z)$, where $a, b$ are gauge indices, that determines the inverse squared gauge coupling constant, is matrix-valued. Throughout this calculation we set

$$
f_{a b}(z)=\delta_{a b} f(z) \equiv \delta_{a b}(x+i y)
$$

The Yang-Mills gauge fixing prescription is modified when $x \neq$ constant, and, since we are now including background as well as quantum Yang-Mills fields, gauge-graviton ghost mixing must be included. We discuss only gauge fixing of the bosonic sector in this section. The fermion sector gauge fixing is unchanged from that defined in $I$, and is summarized in Appendix B.2. Our gauge sign conventions are those of [42] and are defined in Appendix A.

The gauge-fixed Lagrangian (incorporating also the gravity gauge fixing (4.2)) is defined by

$$
\begin{gathered}
\mathcal{L} \rightarrow \mathcal{L}+\mathcal{L}_{g f}, \quad \mathcal{L}_{g f}=-\frac{\sqrt{g}}{2} C_{A} Z^{A B} C_{B}, \quad Z=\left(\begin{array}{cc}
\delta^{a b} & 0 \\
0 & -g^{\mu \nu}
\end{array}\right), \quad C=\left(\begin{array}{c}
C_{a} \\
C_{\mu}
\end{array}\right), \\
C^{a}=\mathcal{D}^{\prime \mu} \hat{\mathcal{A}}_{\mu}^{a}+\frac{i}{\sqrt{x}} K_{i \bar{m}}\left[\left(T^{a} \bar{z}\right)^{\bar{m}} \hat{z}^{i}-\left(T^{a} z\right)^{i} \hat{z}^{\bar{m}}\right], \\
\sqrt{2} C_{\mu}=\left(\nabla^{\nu} h_{\mu \nu}-\frac{1}{2} \nabla_{\mu} h_{\nu}^{\nu}-2 \mathcal{D}_{\mu} z^{I} Z_{I J} \hat{z}^{J}+2 \mathcal{F}_{\mu \nu}^{a} \hat{\mathcal{A}}_{a}^{\nu}\right)
\end{gathered}
$$

where hatted variables refer to quantum fields and unhatted ones refer to background fields, $h_{\mu \nu}$ is the quantum part of the space-time metric whose classical part is $g_{\mu \nu}$, and $K_{i \bar{m}}$ is the Kähler metric, which here is a function of the background fields. Following [41] we have introduced canonically normalized Yang-Mills fields:

$$
\mathcal{A}_{\mu}=\sqrt{x} A_{\mu}, \quad \hat{\mathcal{A}}_{\mu}=\sqrt{x} \hat{A}_{\mu}, \quad \mathcal{F}_{\mu \nu}=\sqrt{x} F_{\mu \nu}, \quad \sqrt{x} \mathcal{D}_{\mu} A_{\nu}=\mathcal{D}_{\mu}^{\prime} \mathcal{A}_{\mu}
$$

and we have adopted the shorthand notation

$$
\mathcal{D}_{\mu}^{\prime}=\mathcal{D}_{\mu}-\frac{\partial_{\mu} x}{2 x}, \quad \mathcal{D}_{\mu}^{\prime \prime}=\mathcal{D}_{\mu}+\frac{\partial_{\mu} x}{2 x}
$$


where $\mathcal{D}_{\mu}$ is the gauge and general coordinate invariant derivative. Under a gauge transformation with parameter $\beta=T_{a} \beta^{a}$ and fixed background fields we have, neglecting terms of order $\hat{z}, \hat{A}$ :

$$
\delta \hat{z}^{i}=-i(\beta z)^{i}, \quad \delta \hat{z}^{\bar{m}}=+i(\beta \bar{z})^{\bar{m}}, \quad \delta \hat{\mathcal{A}}_{\mu}^{a}=\sqrt{x} \mathcal{D}_{\mu} \beta^{a}
$$

If we implement the gauge fixing condition in the usual way, the ghost determinant contains a factor Det $\frac{1}{2} x$ that translates into a quartically divergent term proportional to $\operatorname{Tr} \ln x$ in the effective action. Note however that we have rescaled the quantum YangMills fields [41] [see (4.15) above] and the quantum gaugino fields [37] (see Appendix B.2 below) in order to canonically normalize their kinetic energy. If we rescale the gauge parameter in the same way as the Yang-Mills supermultiplet, and take, instead of $\beta$, the gauge parameter

$$
\gamma=\sqrt{x} \beta, \quad \sqrt{x} \mathcal{D}_{\mu} \beta=\mathcal{D}_{\mu}^{\prime} \gamma
$$

we get

$$
\delta \hat{\mathcal{A}}_{\mu}=\mathcal{D}_{\mu}^{\prime} \gamma, \quad \delta \hat{z}^{i}=-\frac{i}{\sqrt{x}}(\gamma z)^{i}, \quad \delta \hat{z}^{\bar{m}}=+\frac{i}{\sqrt{x}}(\gamma \bar{z})^{\bar{m}},
$$

and no $\operatorname{Tr} \ln x$ term is generated in the ghost determinant. We therefore adopt the prescription (4.18).

Under a general coordinate transformation $x \rightarrow x^{\prime}=x+\epsilon$, we have

$$
\delta \hat{z}^{i}=\epsilon^{\mu} \partial_{\mu} z^{i}, \quad \delta \hat{\mathcal{A}}_{\nu}=\sqrt{x}\left(\epsilon^{\sigma} \nabla_{\sigma} A_{\nu}+A_{\sigma} \nabla_{\nu} \epsilon^{\sigma}\right)
$$

which is general coordinate, but not gauge, covariant. To obtain a manifestly gauge covariant result, we add a compensating gauge transformation with parameter $\gamma^{a}\left(\epsilon^{\mu}\right)=$ $-\epsilon^{\mu} \mathcal{A}_{\mu}^{a}$, giving

$$
\delta \hat{z}^{i}=\epsilon^{\mu} \mathcal{D}_{\mu} z^{i}, \quad \delta \hat{\mathcal{A}}_{\nu}=\epsilon^{\sigma} \mathcal{F}_{\sigma \nu}
$$

Then, relabelling the gauge parameter as $\epsilon_{a} \equiv \gamma_{a}$, the ghost determinant $M$ is obtained in the usual way as

$$
M_{B}^{A}=\frac{\partial}{\partial \epsilon_{A}} \delta C_{B},
$$

where the variation $\delta C$ is determined from

$$
\begin{gathered}
\delta \hat{z}^{i}=-\frac{i}{\sqrt{x}}\left(T_{b} z\right)^{i} \epsilon^{b}+\epsilon^{\mu} \mathcal{D}_{\mu} z^{i}, \quad \delta \hat{z}^{\bar{m}}=\frac{i}{\sqrt{x}}\left(T_{b} \bar{z}\right)^{\bar{m}} \epsilon^{b}+\epsilon^{\mu} \mathcal{D}_{\mu} \bar{z}^{\bar{m}}, \\
\delta \hat{\mathcal{A}}_{\mu}^{a}=\mathcal{D}_{\mu}^{\prime} \epsilon^{a}+\epsilon^{\sigma} \mathcal{F}_{\sigma \mu}^{a}, \quad \delta h_{\mu \nu}=\nabla_{\nu} \epsilon_{\mu}+\nabla_{\mu} \epsilon_{\nu} .
\end{gathered}
$$


This gives a contribution to the gauge-fixed Lagrangian:

$$
\begin{aligned}
g^{-\frac{1}{2}} \mathcal{L}_{g h}= & \bar{c}^{B} M_{B}^{A} c_{A}^{0} \equiv \bar{c} Z\left(\hat{D}^{2}+H_{g h}\right) c \\
= & \bar{c}^{b}\left[\left(\mathcal{D}_{\mu}^{\prime \prime} \mathcal{D}^{\prime \mu}\right)_{b}^{a}+q_{I}^{a} q_{b}^{I}\right] c_{a}-\bar{c}^{\nu} \sqrt{2}\left[\mathcal{D}^{\prime \mu} \mathcal{F}_{\nu \mu}^{a}+q_{I}^{a}\left(\mathcal{D}_{\nu} z^{I}\right)\right] c_{a} \\
& -\bar{c}^{\mu}\left[\nabla^{2} g_{\mu \nu}-r_{\mu \nu}-2\left(\mathcal{D}_{\mu} z^{I}\right) Z_{I J}\left(\mathcal{D}_{\nu} z^{J}\right)+2 \mathcal{F}_{\mu \rho}^{a} \mathcal{F}_{a \nu}{ }^{\rho}\right] c^{\nu} \\
& -\bar{c}^{a} \sqrt{2}\left[\left(\mathcal{D}_{\mu} z^{I}\right) q_{a I}-\mathcal{F}_{a \mu \nu} \mathcal{D}^{\prime \nu}\right] c^{\mu}, \quad c_{0}^{a}=c^{a}, \quad c_{0}^{\mu}=-\sqrt{2} c^{\mu} \\
q_{i}^{a}= & \frac{i}{\sqrt{x}}\left(T^{a} \bar{z}\right)^{\bar{m}} K_{i \bar{m}}, \quad q_{a}^{i}=-\frac{i}{\sqrt{x}}\left(T_{a} z\right)^{i}
\end{aligned}
$$

The rescaling of the graviton ghost in order to canonically normalize the ghost kinetic energy yields a factor $\operatorname{Det}^{-\frac{1}{2}} 2$ in the functional integration that cancels a factor Det $\frac{1}{2} 2$ from the gravitino auxiliary field [37], [30]. The matrix elements of $H_{g h}$ and of the covariant derivative $\hat{D}$ are given in (4.26), (B.29) and (B.30).

Finally, we modify the graviton propagator by adding terms that are proportional to $\mathcal{L}_{A}=\partial \mathcal{L} / \partial \phi^{A}$, where $\phi^{A}$ is any field. This modification, which is equivalent to a nonlinear redefinition of the quantum variables, does not change the S-matrix and can lead to simplifications as well as enhancing manifest covariance under the symmetries of the theory [46]. We define the graviton propagator by

$$
\begin{aligned}
\Delta_{\mu \nu, \rho \sigma}^{-1} & \rightarrow \Delta_{\mu \nu, \rho \sigma}^{-1}-2 P_{\mu \nu, \rho \sigma} \mathcal{L}_{\lambda}^{\lambda}-\frac{1}{2}\left[g_{\mu \nu} \mathcal{L}_{\rho \sigma}+g_{\rho \sigma} \mathcal{L}_{\mu \nu}\right] \\
& +\frac{1}{2}\left[g_{\mu \rho} \mathcal{L}_{\nu \sigma}+g_{\nu \rho} \mathcal{L}_{\mu \sigma}+g_{\mu \sigma} \mathcal{L}_{\nu \rho}+g_{\nu \sigma} \mathcal{L}_{\mu \rho}\right] \\
\Delta_{I \mu \nu}^{-1} & =\frac{1}{\sqrt{g}}\left(D_{I} D_{\mu \nu} S-\frac{1}{2} g_{\mu \nu} D_{I} S\right)
\end{aligned}
$$

and by

$$
\begin{gathered}
\Delta_{\mu \nu, a \rho}^{-1}=\mathcal{L}_{\mu \nu, a \rho}-\frac{1}{2} g_{\mu \nu} \mathcal{L}_{a \rho}+\frac{1}{2} g_{\mu \rho} \mathcal{L}_{a \nu}+\frac{1}{2} g_{\nu \rho} \mathcal{L}_{a \mu}=\mathcal{L}_{\mu \nu ; a \rho}+4 P_{\mu \nu, \rho \sigma} \mathcal{L}_{a}^{\sigma} \\
\mathcal{L}_{\mu \nu, a \rho}=g_{\mu \mu^{\prime}} g_{\nu \nu^{\prime}} g_{\rho \rho^{\prime}} \frac{\partial^{2}}{\partial g_{\mu^{\prime} \nu^{\prime}} \partial A_{\rho^{\prime}}^{a}} \mathcal{L}, \quad \mathcal{L}_{a}^{\sigma}=g^{\sigma \rho} \mathcal{L}_{a \rho}=\frac{\partial}{\partial A_{\sigma}^{a}} \mathcal{L}
\end{gathered}
$$

The spin-2 projection operator is defined as:

$$
P_{\mu \nu, \rho \sigma}=\frac{1}{2}\left(g_{\mu \rho} g_{\nu \sigma}+g_{\nu \rho} g_{\mu \sigma}-g_{\mu \nu} g_{\rho \sigma}\right)=\frac{1}{16} P_{\mu \nu, \rho \sigma}^{-1} .
$$

It should be emphasized that the propagator modifications that we use have been chosen purely for convenience; they considerably simplify the matrix elements that are listed in Appendix B.1, and are not necessarily derivable from a generalized metric [46]. A 
natural choice ${ }^{3}$ for this metric would be $G_{A B}=\sqrt{g}\left(Z_{\Phi}\right)_{A B}$, where $A, B$ run over all bose degrees of freedom and the metric $Z_{\Phi}$ is defined in (4.26) below. Then defining $\Delta_{A B}^{-1}=$ $\mathcal{L}_{A B}-\Gamma_{A B}^{C} \mathcal{L}_{C}$, where $\Gamma_{A B}^{C}$ is the Christoffel connection derived from the metric $G_{A B}$, the propagator corrections would be precisely half the ones used here (with additional corrections to scalar propagator $\Delta_{I J}^{-1}$ and the vector propagator $\Delta_{a \rho, b \sigma}^{-1}$ proportional to $\left.\mathcal{L}_{\mu \nu, \rho \sigma}\right)$. It is possible that the use of this generalized metric would reduce the need for field redefinitions as described in Section 4 [see (4.81-83)], but its use would make the intermediate calculations more cumbersome.

Once the above prescriptions have been implemented, the quadratic quantum Lagrangian for the bosonic sector takes the general form:

$$
\begin{aligned}
\mathcal{L}_{\text {bose }}+\mathcal{L}_{g h}= & -\frac{1}{2} \Phi^{T}\left[Z_{\Phi}\left(D^{2}+M_{\Phi}^{2}\right)+\left\{D_{\mu}, X_{\Phi}^{\mu}\right\}\right] \Phi \\
& +\frac{1}{2} \bar{c}\left[Z_{g h}\left(\mathcal{D}^{2}+M_{g h}^{2}\right)+\left\{\mathcal{D}_{\mu}, X_{g h}^{\mu}\right\}\right] c
\end{aligned}
$$

where $\Phi=\left(h_{\mu \nu}, \hat{\mathcal{A}}^{a}, \hat{z}^{i}, \hat{z}^{\bar{m}}\right), D_{\mu}$ is covariant under scalar field redefinitions as well as gauge and general coordinate transformations, and the $X_{\mu}$ connect fields of different spin; in addition, there is a vector-vector connection [41] in $X_{\Phi}^{\mu}$. Following the procedure described in [41], we introduce off-diagonal connections in both the bosonic and ghost sectors, as well as an additional connection for the gauge fields, so as to cast the quantum Lagrangian for the full gauge-fixed bosonic sector in the form

$$
\begin{aligned}
\mathcal{L}_{\text {bose }}+\mathcal{L}_{g h} & =-\frac{1}{2} \Phi^{T} Z_{\Phi}\left(\hat{D}_{\Phi}^{2}+H_{\Phi}\right) \Phi+\frac{1}{2} \bar{c} Z_{g h}\left(\hat{D}_{g h}^{2}+H_{g h}\right) c \\
\hat{D}_{\mu}^{\Phi} & =D_{\mu}+V_{\mu}, \quad\left(V_{\mu}\right)_{a \rho, b \sigma}=-\delta_{a b} \epsilon_{\rho \mu \sigma \nu} \frac{\partial^{\nu} y}{2 x} \\
\left(Z V_{\mu}\right)_{\alpha \beta, a \nu} & =\left(V_{\mu}\right)_{a \nu, \alpha \beta}=\frac{1}{4}\left(\mathcal{F}_{a \beta \mu} g_{\alpha \nu}+\mathcal{F}_{a \alpha \mu} g_{\beta \nu}\right) \\
\left(V_{\mu}\right)_{a \nu, i} & =\left(V_{\mu}\right)_{i, a \nu}=\left[\left(V_{\mu}\right)_{\bar{\imath}, a \nu}\right]^{*}=\frac{1}{4 x} f_{i}\left(\mathcal{F}_{a \mu \nu}-i \tilde{\mathcal{F}}_{a \mu \nu}\right) \\
\hat{D}_{\mu}^{g h} & =\mathcal{D}_{\mu}+B_{\mu}, \quad\left(B_{\mu}\right)_{a \nu}=\left(B_{\mu}\right)_{\nu a}=-\frac{1}{\sqrt{2}} \mathcal{F}_{a \nu \mu} .
\end{aligned}
$$

This introduces corresponding shifts in the background field-dependent "squared mass" matrices:

$$
M_{\Phi}^{2} \rightarrow H_{\Phi}=M_{\Phi}^{2}-V_{\mu} V^{\mu}, \quad M_{g h}^{2} \rightarrow H_{g h}=M_{g h}^{2}-B_{\mu} B^{\mu}
$$

The elements of $M_{\Phi}^{2}$ were evaluated in [41]; here they are somewhat modified by the

\footnotetext{
${ }^{3}$ This choice for $G^{\mu \nu, \rho \sigma}$ coincides with that of Fradkin and Tseytlin [46] for the case of supergravity with their parameter $t=1$, which corresponds to $\lambda=-1 / 2$ in their pure gravity case.
} 
different Yang-Mills gauge fixing and action expansion. These modified matrix elements are listed in Appendix B.1 below.

As explained in Sections 3.3 and 4.3, we evaluate the fermion determinant by first writing it in two-component notation, separating it into helicity-even and -odd contributions, and then recasting these two contributions in Lorentz covariant four-component notation. As discussed in [45], this separation is not uniquely defined. The choice that respects supersymmetry as well as manifest gauge and Kähler covariance allows a consistent Pauli-Villars regulation. We follow that choice here; the corresponding matrix elements are given in the Appendix B. The contribution from fermion loops to the effective action is evaluated (see Sec. 3.3) by introducing [37] the $8 \times 8$ matrices

$$
D_{\mu}=\left(\begin{array}{cc}
D_{\mu}^{+} & 0 \\
0 & D_{\mu}^{-}
\end{array}\right), \quad M_{\Theta}=\left(\begin{array}{cc}
0 & M \\
\bar{M} & 0
\end{array}\right), \quad \not D=\gamma^{\mu} D_{\mu}
$$

that operate on an eight component fermion $f^{T}=\left(f_{L}, f_{R}=f_{L}^{c}\right)$. The helicity averaged contribution of the fermion determinant is then

$$
-\frac{i}{4} \operatorname{Tr} \ln \left(-i \not D+M_{\Theta}\right)_{+}=-\frac{i}{8} \operatorname{Tr} \ln \left(\not D^{2}+M_{\Theta}^{2}-i\left[\not D, M_{\Theta}\right]\right)
$$

Because the fermion mass matrix and connection contain the terms $\sigma^{\mu \nu} M_{\mu \nu}$ and $i L_{\mu} \gamma_{5}$, respectively, they do not commute with $\gamma_{\mu}$; thus

$$
\begin{aligned}
\not D^{2} & =D^{2}+\frac{1}{4}\left[\gamma^{\mu}, \gamma^{\nu}\right] G_{\mu \nu}+\frac{1}{2}\left\{D_{\nu}, \gamma^{\mu}\left[D_{\mu}, \gamma^{\nu}\right]\right\}-\frac{1}{2}\left[D_{\nu}, \gamma^{\mu}\left[D_{\mu}, \gamma^{\nu}\right]\right] \\
{\left[\not D, M_{\Theta}\right] } & =\frac{1}{2}\left\{\gamma_{\mu}, D^{\mu} M_{\Theta}\right\}+\frac{1}{2}\left\{D^{\mu},\left[\gamma_{\mu}, M_{\Theta}\right]\right\}+\frac{1}{2}\left[M_{\Theta},\left[D^{\mu}, \gamma_{\mu}\right]\right] \\
D^{\mu} M_{\Theta} & \equiv\left[D^{\mu}, M_{\Theta}\right]
\end{aligned}
$$

Therefore, in analogy with the boson case discussed above, we write

$$
\begin{gathered}
-\frac{i}{4} \operatorname{Tr} \ln \left(-i \not D+M_{\Theta}\right)_{+}=-\frac{i}{8} \operatorname{Tr} \ln \left(\hat{D}_{\Theta}^{2}+H_{\Theta}\right) \\
H_{\Theta}=M_{\Theta}^{2}-\frac{i}{2}\left\{\gamma^{\mu}, D_{\mu} M_{\Theta}\right\}+\frac{1}{4}\left[\gamma^{\mu}, M_{\Theta}\right]\left[\gamma_{\mu}, M_{\Theta}\right]-\frac{i}{2}\left[M_{\Theta},\left[D^{\mu}, \gamma_{\mu}\right]\right]+\frac{1}{4}\left[\gamma^{\mu}, \gamma^{\nu}\right] G_{\mu \nu} \\
\quad-\frac{1}{4} \gamma^{\mu}\left[D_{\mu}, \gamma^{\nu}\right] \gamma^{\rho}\left[D_{\rho}, \gamma_{\nu}\right]-\frac{1}{2}\left[D_{\nu}, \gamma^{\mu}\left[D_{\mu}, \gamma^{\nu}\right]\right]+\frac{i}{4}\left\{\left[\gamma^{\mu}, M_{\Theta}\right], \gamma^{\nu}\left[D_{\nu}, \gamma_{\mu}\right]\right\} \\
\hat{D}_{\mu}^{\Theta}=D_{\mu}-\frac{i}{2}\left[\gamma_{\mu}, M_{\Theta}\right]+\frac{1}{2} \gamma^{\nu}\left[D_{\nu}, \gamma_{\mu}\right] .
\end{gathered}
$$




\subsection{Helicity-Odd Fermion Loop Contributions}

In this section we determine the helicity-odd operators that arise from integration over fermionic degrees of freedom. They are particularly relevant to the evaluation of anomalies [26], [29], in effective supergravity theories, which is currently of special interest in attempts to extract physics from string theory. We show that these terms are finite, except in the presence of a Yang-Mills sector with a nontrivial kinetic normalization function $f(z)$, in which case there are logarithmically divergent contributions that are invariant under chiral $U(1)_{R}$ transformations, i.e., under Kähler (or modular) transformations up to a possible dependence of the cut-off on the Kähler potential. We also indicate how the finite contributions to the effective action can be obtained.

\subsubsection{General formalism}

The fermion loop contribution is given by

$$
\mathcal{L}_{1}=-\frac{i}{2} \operatorname{Tr} \ln \left(-i \not D+M_{\Theta}\right) \equiv-\frac{i}{2} \operatorname{Tr} \ln \mathcal{M}
$$

To evaluate the determinant (4.33), we write

$$
T=\operatorname{Tr} \ln \mathcal{M}=T_{+}+T_{-}, \quad T_{ \pm}=\frac{1}{2}\left[\operatorname{Tr} \ln \mathcal{M}\left(\gamma_{5}\right) \pm \operatorname{Tr} \ln \mathcal{M}\left(-\gamma_{5}\right)\right]
$$

Only $T_{+}$has been calculated previously for supergravity [30]-[40]. Here we will evaluate the additional contribution, $T_{-}$:

$$
\begin{gathered}
T_{-}=-\frac{1}{2} \operatorname{Tr} \ln \mathcal{M}\left(-\gamma_{5}\right) \mathcal{M}^{-1}\left(\gamma_{5}\right)=-\frac{1}{2} \operatorname{Tr} \ln \left\{1-\mathcal{M}^{-1}\left[\mathcal{M}\left(\gamma_{5}\right)-\mathcal{M}\left(-\gamma_{5}\right)\right]\right\} \\
=\frac{1}{2} \operatorname{Tr} \sum_{n=1}^{\infty} \frac{1}{n}\left\{\mathcal{M}^{-1}\left[\mathcal{M}\left(\gamma_{5}\right)-\mathcal{M}\left(-\gamma_{5}\right)\right]\right\}^{n} .
\end{gathered}
$$

Using the techniques described in [48], [37], we can write the trace in (4.35) as (seeSection 3.3)

$$
T_{-}=\int d^{4} x T(x), \quad T(x)=\int \frac{d^{4} p}{(2 \pi)^{4}} T(p, x),
$$

and then expand $T(p, x)$ as

$$
T(p, x)=\operatorname{Tr} \sum_{n=1}^{\infty} \frac{2^{n}}{2 n}\left\{\sum_{\ell=0}^{\infty}(-\mathcal{R})^{\ell} \mathcal{R}_{5}\right\}^{n},
$$


where $\mathcal{R}, \mathcal{R}_{5}$ are defined in (3.33-34):

$$
\begin{aligned}
\mathcal{R} & =\frac{1}{-p^{2}}\left[p^{2}-T^{\mu \nu} \Delta_{\mu} \Delta_{\nu}+\hat{h}+X+\left(p^{\nu}+G^{\nu}\right) P_{\mu \nu} \widehat{M}^{\mu}\right] \\
\mathcal{R}_{5} & =\frac{1}{-p^{2}}\left[\left(p^{\nu}+G^{\nu}\right) P_{\mu \nu} \hat{N}^{\mu}\right] .
\end{aligned}
$$

The operators appearing in (4.38) are defined in Section 3.3 as power series of the form $\sum_{n} c_{n}(O)(D \cdot \partial / \partial p)^{n} O$, where $D_{\mu}=D_{\mu}^{+} R+D_{\mu}^{-} L$ is the fully covariant derivative defined in (3.22) of the Appendix, and the operator $O$ is a function of the background bosons. The coefficients $c_{n}(O)$ are constants with, in particular, $c_{0}(G)=0$ in the expansion of $G_{\mu}^{ \pm}$; more specifically

$$
G^{ \pm}=\gamma^{\mu} G_{\mu}^{ \pm} \quad G_{\mu}^{ \pm}=\frac{1}{2} G_{\nu \mu}^{ \pm} \frac{\partial}{\partial p_{\nu}}+O\left(\frac{\partial^{2}}{\partial p \partial p}\right), \quad G_{\mu \nu}^{ \pm}=-G_{\nu \mu}^{ \pm}=\left[D_{\mu}^{ \pm}, D_{\nu}^{ \pm}\right]
$$

Thus we have to evaluate the following contribution to the effective one-loop Lagrangian:

$$
\mathcal{L}_{1} \ni-\frac{i}{2} T_{-}=-i \int \frac{d^{4} p}{4(2 \pi)^{4}} \operatorname{Tr} \sum_{n=1}^{\infty} \frac{2^{n}}{n}\left\{\sum_{\ell=0}^{\infty}(-\mathcal{R})^{\ell} \mathcal{R}_{5}\right\}^{n}
$$

where now the trace is over only Dirac indices and internal quantum numbers (and Lorentz indices for the gravitino).

To keep the integrals finite, the integration should be performed including PauliVillars regulator masses $\mu_{0}:-p^{-2} \rightarrow\left(-p^{2}+\mu_{0}^{2}\right)^{-1}$ in the derivative expansion. However, as shown below, $T_{-}$, when suitably defined, contains no quadratically divergent terms. Once the integrals are properly regulated-including the appropriate definitions of $T_{ \pm}-$the coefficients of log divergent terms are independent of the regularization scheme. On the other hand, if one wishes to evaluate finite terms, one has either to expand around an infrared regulator mass $\mu_{0}$ or, alternatively, to resum the derivative expansion [51] [52]. In particular, the ultra-violet finite terms include the standard chiral anomaly. We explicitly evaluated this term for the vector-vector-axial vertex induced by Dirac fermions with a common mass $\mu_{0}$, and recovered the large mass limit of the Adler-Rosenberg formula [53]; the complete expression for this formula requires a resummation of the derivative expansion which will be presented elsewhere [52]. We emphasize that, because of the anomaly, Kähler invariance is broken at the quantum level. Classically, this invariance permits a choice [42] of Kähler gauge such that the classical Lagrangian is derivable from only two functions of the scalar fields, the (in general matrix-valued) gauge normalization function $f_{a b}(z)$ and the generalized Kähler potential $\mathcal{G}(z, \bar{z})=K(z, \bar{z})+\ln |W(z)|^{2}$, 
where $K$ and $W$ are the Kähler potential and the superpotential, respectively. For the purpose of calculating the anomaly [26], [29], one has to undo the Kähler rotation of Cremmer et al. [42], by performing a phase transformation [13] on the fermion fields. We work throughout in this Kähler covariant formalism.

As was discussed in [45], the separation (4.34) of $T$ into helicity-odd and -even parts is not uniquely defined because we can interchange terms that are even and odd in $\gamma_{5}$ using $\gamma_{5}=(i / 24) \epsilon^{\mu \nu \rho \sigma} \gamma_{\mu} \gamma_{\nu} \gamma_{\rho} \gamma_{\sigma}$ and similar identities. In most cases the correct choice is dictated by gauge or Kähler covariance. The remaining ambiguities are resolved by supersymmetry. A fully SUSY-invariant result for the quadratically divergent terms requires the introduction of Pauli-Villars regulator fields [40], [49]; there is a unique definition of the matrix elements that allows a supersymmetric Pauli-Villars regularization [45]. Specifically, this fixes the forms of the fermion mass matrix and connection matrix:

$$
\begin{aligned}
M & =m+\left(\alpha_{a} F_{\mu \nu}^{a}+i \beta_{a} \gamma_{5} \tilde{F}_{\mu \nu}^{a}\right) \sigma^{\mu \nu}, \quad \tilde{F}_{\mu \nu}=\frac{1}{2} \epsilon_{\mu \nu \rho \sigma} F^{\rho \sigma} \\
D_{\mu} & =\mathcal{D}_{\mu}+i \Gamma_{\mu} \gamma_{5}-\frac{1}{24} L_{\mu} \epsilon^{\lambda \nu \rho \sigma} \gamma_{\lambda} \gamma_{\nu} \gamma_{\rho} \gamma_{\sigma},
\end{aligned}
$$

where $\Gamma_{\mu}, L_{\mu}, m$, and $\alpha, \beta$ are proportional to the unit matrix in Dirac space. $\mathcal{D}_{\mu}$, which contains the spin connection, is the gauge and general coordinate covariant derivative, $\Gamma_{\mu}$ is the Kähler connection, $F_{\mu \nu}$ is the Yang-Mills field strength, and $L_{\mu}$ is an additional axial connection for gauginos arising from the noncanonical form of the kinetic energy term. $T_{ \pm}$are defined by (4.34) using the explicit $\gamma_{5}$-dependence in (4.41). Then the operators appearing in the derivative expansion of (4.38-39) take the form:

$$
\begin{aligned}
G_{\mu \nu}^{ \pm} & =\tilde{G}_{\mu \nu}^{ \pm}+i \gamma_{5} L_{\mu \nu}^{ \pm}-\left[L_{\mu}, L_{\nu}\right], \quad \tilde{G}_{\mu \nu}^{ \pm}=\left[\tilde{D}_{\mu}^{ \pm}, \tilde{D}_{\nu}^{ \pm}\right], \\
\tilde{D}_{\mu}^{ \pm} & =\mathcal{D}_{\mu}^{ \pm} \pm i \Gamma_{\mu}+\Gamma_{\mu}^{\prime}, \quad L_{\mu \nu}^{ \pm}=\tilde{D}_{\mu}^{ \pm} L_{\nu}-\tilde{D}_{\nu}^{ \pm} L_{\mu}, \quad \tilde{D}_{\mu}^{ \pm} L_{\nu} \equiv\left[\tilde{D}_{\mu}^{ \pm}, L_{\nu}\right], \\
\mathcal{J}_{\mu} & =\frac{i}{2}\left(\tilde{D}_{\mu}^{+}-\tilde{D}_{\mu}^{-}\right)=\frac{i}{2}\left(\mathcal{D}_{\mu}^{+}-\mathcal{D}_{\mu}^{-}\right)-\Gamma_{\mu}, \quad M_{I}=\frac{1}{2}(M-\bar{M}), \\
M & =m+M_{\sigma}=m+M_{\mu \nu} \sigma^{\mu \nu}, \quad \bar{M}=\bar{m}+\bar{M}_{\sigma}=\bar{m}+\bar{M}_{\mu \nu} \sigma^{\mu \nu}, \\
M_{\mu \nu} & =\alpha F_{\mu \nu}-i \beta \tilde{F}_{\mu \nu}, \quad \bar{M}_{\mu \nu}=\bar{\alpha} F_{\mu \nu}+i \bar{\beta} \tilde{F}_{\mu \nu},
\end{aligned}
$$

where $\Gamma_{\mu}$ is the Kähler connection and $\Gamma_{\mu}^{\prime}$ is an off-diagonal $\lambda-\psi$ connection. We consider only the case where the gauge field normalization function $f(z)$ is diagonal in gauge indices; then, since $\Gamma_{\mu}$ is diagonal, $L_{\mu}$ commutes with $\mathcal{J}_{\nu}$, and we have

$$
\begin{aligned}
& L_{\mu \nu}^{+}=L_{\mu \nu}^{-} \equiv \hat{L}_{\mu \nu}=L_{\mu \nu}+\left[\Gamma_{\mu}^{\prime}, L_{\nu}\right]-\left[\Gamma_{\nu}^{\prime}, L_{\mu}\right], \\
& L_{\mu \nu}=\nabla_{\mu} L_{\nu}-\nabla_{\nu} L_{\mu}, \quad\left[L_{\mu}, L_{\nu}\right]=0 .
\end{aligned}
$$


Note that the spin connection in $\tilde{D}_{\mu}$ drops out of the covariant derivatives $\tilde{D}_{\mu} M$. This is because we have taken the vierbein, and therefore $\gamma_{\mu}$, to be covariantly constant [54]: $\left[\tilde{D}_{\mu}, \gamma_{\nu}\right]=0$. The spin connection is even in $\gamma_{5}$ and therefore contributes to $\tilde{D}_{\mu} M$ through the commutator which vanishes [see the definitions (4.59) below].

To identify the ultraviolet divergences, we have to study the large $p$ behavior of the integrand in (4.40) and keep terms up to $O\left(p^{-4}\right)$. A priori $\mathcal{R}, \mathcal{R}_{5} \sim p^{-1}$, so the ultraviolet divergent part of (4.39) can occur only in terms with $n \leq 4, \ell \leq 4-n$. Aside from terms involving $L_{\mu}$, by construction, the integrand is odd in $\gamma_{5}$, and we need at least four $\gamma_{\mu}$ 's to get a nonvanishing trace:

$$
T \propto \operatorname{Tr}\left(A_{\mu \nu \rho \sigma} \gamma^{\mu} \gamma^{\nu} \gamma^{\rho} \gamma^{\sigma} \gamma^{5}\right)=-4 i \epsilon^{\mu \nu \rho \sigma} \operatorname{Tr} A_{\mu \nu \rho \sigma}
$$

so $\operatorname{Tr} \mathcal{R}_{5}=0$. Finally, we note that $G_{\mu}^{ \pm}$in (4.39) vanishes except when sandwiched between functions of $p$, and is of order $p^{-1}$ in power counting. Once all $p$-differentiations have been performed, surviving terms must have at least three $\gamma_{\mu}$ 's that are not contracted with $p^{\mu}$ because of antisymmetry. After integration over $p$, the tensor $A_{\mu \nu \rho \sigma}$ in (4.44) can be constructed only from the four-vectors $\mathcal{J}_{\mu}$ and $L_{\mu}$, the tensors $M_{\mu \nu}, G_{\mu \nu}^{ \pm}$, the Riemann tensor, and their covariant derivatives $\tilde{D}_{\mu}$. Each factor of $G_{\mu \nu}^{ \pm}$and of $D_{\mu}$ reduces the apparent divergence of a given term by one power of $p$. Furthermore, in the covariant derivative expansions (3.33-34) of the operators $O$ appearing in (4.37) the indices $\mu_{i} \cdots \mu_{n}$ in $D_{\mu_{i}} \cdots D_{\mu_{n}} O$ are automatically symmetrized, so at most one derivative of each operator can contribute to $A_{\mu \nu \rho \sigma}$ in (4.44).

\subsubsection{Quadratically divergent contributions}

By construction, $T_{-}$is antisymmetric under $\gamma_{5} \rightarrow-\gamma_{5}$. Therefore we can evaluate, instead of (4.37)

$$
T_{-} \rightarrow \frac{1}{2}\left[T_{-}\left(\gamma_{5}\right)-T_{-}\left(-\gamma_{5}\right)\right]
$$

where $T_{-}\left(-\gamma_{5}\right)$ is obtained from $T_{-}\left(\gamma_{5}\right)$ by the substitutions

$$
\left(D^{+}, D^{-}, M, \bar{M}, \mathcal{J}, M_{I}\right) \rightarrow\left(D^{-}, D^{+}, \bar{M}, M,-\mathcal{J},-M_{I}\right) .
$$

The matrices $\mathcal{R}, \mathcal{R}_{5}$ are defined in (3.33-34). Since $\int d^{4} p \operatorname{Tr} \mathcal{R}_{5}=0$, the potentially quadratically divergent contribution to $T_{-}$is

$$
\operatorname{Tr}\left(\mathcal{R}_{5}^{2}-\mathcal{R} \mathcal{R}_{5}\right) \rightarrow \frac{1}{p^{4}} \operatorname{Tr}\left[\left(p^{\mu} N_{\mu}-p^{\mu} M_{\mu}\right) p^{\nu} N_{\nu}\right]
$$


with $N_{\nu}, M_{\nu}$ given in (3.29). Under Lorentz invariant integration, with $M=m+$ $\sigma_{\mu \nu} M^{\mu \nu}$, we have

$$
\int d^{4} p \not p M \not p M^{\prime}\left(1 \pm \gamma_{5}\right) \propto \int d^{4} p p^{2} \gamma_{\mu} M \gamma^{\mu} M^{\prime}\left(1 \pm \gamma_{5}\right)=4 \int d^{4} p m \not p M^{\prime}\left(1 \pm \gamma_{5}\right)
$$

It follows that there are no quadratically divergent contribution involving the mass matrix. The averaging procedure (4.45) eliminates a residual spurious quadratic divergence proportional to $\operatorname{Tr} \mathcal{J}_{\mu} \mathcal{J}^{\mu}$. This divergence would vanish identically if a Pauli-Villars regularization were used with $\mathrm{P}-\mathrm{V}$ masses that leave all classical symmetries unbroken. However this is not in general possible for the classical Kähler symmetry. ${ }^{4}$ Moreover, in the Pauli-Villars regularization described in [45], there are no P-V fields that can regulate quadratic divergences proportional to $M_{\mu \nu} M^{\mu \nu}$, so the integrals, which are illdefined unless they are explicitly regulated, must be defined in such a way that these divergences do not appear. Note that no quadratically divergent contribution to $T_{-}$ arises if (4.35), as defined by (3.20), is expanded without performing the the transformation (3.30) that makes use of partial integration, which is ill-defined if the integrals are not finite. However this transformation renders many terms explicitly covariant and thereby considerably simplifies the derivative expansion.

\subsubsection{Logarithmically divergent contributions}

In the remainder of this section, $T_{-}$is understood as the average (3.45). Since we encounter only logarithmic divergences, after symmetric integration we may make the replacements:

$$
\begin{aligned}
p_{\mu} p_{\nu} f\left(p^{2}\right) & \rightarrow \frac{p^{2}}{4} g_{\mu \nu} f\left(p^{2}\right) \\
p_{\mu} p_{\nu} p_{\rho} p_{\sigma} f\left(p^{2}\right) & \rightarrow \frac{p^{4}}{24}\left(g_{\mu \nu} g_{\rho \sigma}+g_{\mu \rho} g_{\nu \sigma}+g_{\mu \sigma} g_{\nu \rho}\right) f\left(p^{2}\right)
\end{aligned}
$$

To evaluate the terms with $p$-derivatives, we write

$$
\begin{aligned}
& \frac{1}{-p^{2}} p^{\mu} \frac{\partial}{\partial p_{\nu}} \rightarrow-\frac{1}{-p^{2}} A^{\mu \nu}, \quad \frac{1}{-p^{2}} p^{\mu} G_{\nu \mu} \frac{\partial}{\partial p_{\nu}} \rightarrow 0, \quad A^{\mu \nu}=g^{\mu \nu}-\frac{2}{p^{2}} p^{\mu} p^{\nu} \\
& \frac{\partial}{\partial p_{\nu}} \frac{1}{-p^{2}} p^{\mu} \rightarrow \frac{1}{-p^{2}} A^{\mu \nu}, \quad p^{\mu} G_{\nu \mu} \frac{\partial}{\partial p_{\nu}} \frac{1}{-p^{2}} p^{\rho} \rightarrow \frac{1}{-p^{2}} p^{\mu} G_{\nu \mu} g^{\rho \nu}
\end{aligned}
$$

where the first line is obtained by partial integration over $p$, and it is understood that operators multiplying the first (second) line on the left (right) are independent of $p$.

\footnotetext{
${ }^{4} \mathrm{~A}$ detailed discussion of Pauli-Villars regularization of $T_{-}$will be given elsewhere [52].
} 
Similarly

$$
\begin{aligned}
\frac{\partial^{2}}{\partial p_{\mu} \partial p_{\nu}} \frac{1}{-p^{2}} p^{\rho} & \rightarrow \frac{2}{p^{4}}\left(g^{\rho \mu} p^{\nu}+g^{\rho \nu} p^{\mu}+g^{\mu \nu} p^{\rho}-\frac{4}{p^{2}} p^{\mu} p^{\nu} p^{\rho}\right) \\
\frac{\partial^{2}}{\partial p_{\mu} \partial p_{\nu}} \frac{1}{-p^{2}} \not p^{\rho} p^{\sigma} r_{\mu \rho \sigma \nu} & \rightarrow \frac{1}{-p^{2}}\left(r \not p-\frac{2}{p^{2}} p^{\mu} p^{\nu} \not p r_{\mu \nu}+2 r^{\mu \nu} \gamma_{\mu} p_{\nu}\right) \\
\frac{1}{-p^{2}} \frac{\partial^{2}}{\partial p_{\mu} \partial p_{\nu}} p^{\rho} p^{\sigma} r_{\mu \rho \sigma \nu} & \rightarrow-\frac{2}{p^{4}} p^{\mu} p^{\nu} r_{\mu \nu},
\end{aligned}
$$

where the last line is obtained by partial integration.

It is easy to see that the nonvanishing terms in $T_{-}$involve the connection $L_{\mu}$ and/or the off-diagonal mass $M_{\mu \nu}$. In the absence of these contributions, since $\epsilon^{\mu \nu \rho \sigma} r_{\mu \nu \rho \tau}=0$, the only helicity-odd terms are:

$$
\epsilon^{\mu \nu \rho \sigma} \operatorname{Tr}\left[\left(D_{\mu}^{e} \mathcal{J}_{\nu}\right) \mathcal{J}_{\rho} \mathcal{J}_{\sigma}\right], \quad \epsilon^{\mu \nu \rho \sigma} \operatorname{Tr}\left[G_{\mu \nu}^{A} \mathcal{J}_{\rho} \mathcal{J}_{\sigma}\right], \quad \epsilon^{\mu \nu \rho \sigma} \operatorname{Tr}\left[G_{\mu \nu}^{V} D_{\rho}^{e} \mathcal{J}_{\sigma}\right],
$$

where

$$
D_{\mu}^{e}=\frac{1}{2}\left(D_{\mu}^{+}+D_{\mu}^{-}\right) \equiv \partial_{\mu}+\mathcal{J}_{\mu}^{\prime}, \quad G_{\mu \nu}^{A(V)}=\frac{1}{2}\left[G_{\mu \nu}^{+}-(+) G_{\mu \nu}^{-}\right] .
$$

The first term in (4.50) can be written

$$
\frac{1}{3} \epsilon^{\mu \nu \rho \sigma} \operatorname{Tr}\left[D_{\mu}^{e}\left(\mathcal{J}_{\nu} \mathcal{J}_{\rho} \mathcal{J}_{\sigma}\right)\right]=\frac{1}{3} \epsilon^{\mu \nu \rho \sigma} \partial_{\mu}\left(\operatorname{Tr}\left[\mathcal{J}_{\nu} \mathcal{J}_{\rho} \mathcal{J}_{\sigma}\right]\right),
$$

where we used cyclic permutations in the trace together with the relation

$$
\operatorname{Tr}\left[D_{\mu}^{e}(\mathcal{J J J})\right]=\operatorname{Tr}\left\{\partial_{\mu}(\mathcal{J J J J})+i\left[\mathcal{J}_{\mu}^{\prime}, \mathcal{J} \mathcal{J J}\right]\right\}=\partial_{\mu} \operatorname{Tr}(\mathcal{J J J})
$$

Note that if a field-dependent ultraviolet regulator mass $\Lambda$ is present one cannot drop the total derivative on the right hand side of (4.51), but integrating by parts gives $\partial \ln \Lambda=\partial \Lambda / \Lambda$ which is finite for $\Lambda \rightarrow \infty$. For the second term in (4.50), defining $D_{\mu}^{ \pm}=\partial_{\mu}+\Gamma_{\mu}^{ \pm}$, we have

$$
G_{\mu \nu}^{ \pm}=\partial_{\mu} \Gamma_{\nu}^{ \pm}-\partial_{\nu} \Gamma_{\mu}^{ \pm}+\left[\Gamma_{\mu}^{ \pm}, \Gamma_{\nu}^{ \pm}\right]=D_{\mu} \Gamma_{\nu}^{ \pm}-D_{\nu} \Gamma_{\mu}^{ \pm}-\left[\Gamma_{\mu}^{ \pm}, \Gamma_{\nu}^{ \pm}\right] .
$$

By the above argument the $D \Gamma$ terms give finite contributions, so we are left with

$$
\epsilon^{\mu \nu \rho \sigma} \operatorname{Tr}\left[\left(\Gamma_{\mu}^{+} \Gamma_{\nu}^{+}-\Gamma_{\mu}^{-} \Gamma_{\nu}^{-}\right)\left(\Gamma_{\rho}^{+}-\Gamma_{\rho}^{-}\right)\left(\Gamma_{\sigma}^{+}-\Gamma_{\sigma}^{-}\right)\right]=0,
$$

again using cyclic permutations of the trace. Since $\epsilon^{\mu \nu \rho \sigma} D_{\mu}^{e}\left[D_{\nu}^{e}, D_{\rho}^{e}\right]$ vanishes by virtue of the Bianchi identity, the third term in (4.50) reduces (up to a total derivative) to the same form as the first term: $G^{V} \rightarrow[\mathcal{J}, \mathcal{J}]$. 
First consider the terms quartic in $\mathcal{R}, \mathcal{R}_{5}$. To obtain the logarithmically divergent piece, we drop all $p$-derivatives:

$$
\mathcal{R} \rightarrow \frac{1}{-p^{2}} p_{\mu} M^{\mu}, \quad \mathcal{R}_{5} \rightarrow \frac{1}{-p^{2}} p_{\mu} N^{\mu}
$$

We note that $F_{a}^{\mu \nu} F_{\nu \rho}^{b} F_{\mu}^{c \rho}$ and $F_{a}^{\mu \nu} F_{\nu \rho}^{b} \tilde{F}_{\mu}^{c \rho}$ vanish if any two of the indices $a, b, c$ are equal; there are therefore no terms cubic in $M_{\sigma}$. Then using $\gamma_{\mu} M \gamma^{\mu}=4 m$, together with eqs. (3.37) and (A.12-13) and cyclic permutivity of the trace, we obtain:

$$
\begin{aligned}
H\left(M_{1}, M_{2}\right) \equiv & \operatorname{Tr}\left(\not p M_{1} \not p \not \supset \not M_{2} \not p \not J \gamma_{5}\right) \\
\rightarrow & \frac{16 i}{3} p^{4} \operatorname{Tr}\left(\widetilde{M}_{1}^{\mu \nu} \mathcal{J}_{\nu} M_{\mu \rho}^{2} \mathcal{J}^{\rho}-M_{1}^{\mu \nu} \mathcal{J}_{\nu} \widetilde{M}_{\mu \rho}^{2} \mathcal{J}^{\rho}\right), \\
F\left(M_{1}, M_{2}\right) \equiv & \operatorname{Tr}\left(\not p M_{1} \not p M_{2} \not p \not \supset \not p \not J \gamma_{5}\right) \\
\rightarrow & 4 p^{4} \operatorname{Tr}\left[\left(\widetilde{M}_{1}^{\mu \nu} m_{2}-m_{1} \widetilde{M}_{2}^{\mu \nu}\right) \mathcal{J}_{\mu} \mathcal{J}_{\nu}\right] \\
& +\frac{4 i}{3} p^{4} \operatorname{Tr}\left[\left(M_{\mu \rho}^{1} \widetilde{M}_{2}^{\mu \nu}-\widetilde{M}_{1}^{\mu \nu} M_{\mu \rho}^{2}\right)\left\{\mathcal{J}^{\rho}, \mathcal{J}_{\nu}\right\}\right] \\
F^{\prime}\left(M_{1}, M_{2}, M_{3}, M_{4}\right)= & -F^{\prime}\left(M_{4}, M_{1}, M_{2}, M_{3}\right) \equiv \operatorname{Tr}\left(\not p M_{1} \not p M_{2} \not p M_{3} \not p M_{4} \gamma_{5}\right) \\
& -\frac{16 i}{3} p^{4} \operatorname{Tr}\left(\widetilde{M}_{1}^{\mu \nu} M_{2}^{\rho \sigma} M_{\mu \nu}^{3} M_{\rho \sigma}^{4}-M_{1}^{\mu \nu} M_{2}^{\rho \sigma} M_{\mu \nu}^{3} \widetilde{M}_{\rho \sigma}^{4}\right) \\
& +8 i p^{4} \operatorname{Tr}\left(m_{1} M_{\mu \nu}^{2} m_{3} \widetilde{M}_{4}^{\mu \nu}-m_{4} M_{\mu \nu}^{1} m_{2} \widetilde{M}_{3}^{\mu \nu}\right),
\end{aligned}
$$

where $M_{i}=M, \bar{M}, M_{I}, \widetilde{M}_{i}^{\mu \nu}=\frac{1}{2} \epsilon^{\mu \nu \rho \sigma}\left(M_{i}\right)_{\rho \sigma}$, and the traces on the right hand sides are over internal indices only. In evaluating these expressions we used the fact that since $\operatorname{Tr}\left(M_{\sigma}^{1} M_{\sigma}^{2} M_{\sigma}^{3} M_{\sigma}^{4} \gamma_{5}\right)=\operatorname{Tr}\left(M_{\sigma}^{4} M_{\sigma}^{1} M_{\sigma}^{2} M_{\sigma}^{3} \gamma_{5}\right)$, these terms do not contribute to

$$
\frac{1}{2}\left[F^{\prime}\left(M_{1}, M_{2}, M_{3}, M_{4}\right)-F^{\prime}\left(M_{4}, M_{1}, M_{2}, M_{3}\right)\right]=F^{\prime}\left(M_{1}, M_{2}, M_{3}, M_{4}\right) .
$$

Finally, since the expression (4.38) for $\mathcal{R}_{5}$ is odd in $\gamma_{5}:\left[\mathcal{R}_{5}\left(\gamma_{5}\right)\right]^{4}=+\left[\mathcal{R}_{5}\left(-\gamma_{5}\right)\right]^{4}$, it follows that $\operatorname{Tr}\left(\mathcal{R}_{5}\right)^{4}$ does not contribute to $T\left(\gamma_{5}\right)=-T\left(-\gamma_{5}\right)$. The logarithmically divergent contributions from the quartic terms in (4.40) are therefore given by:

$$
\begin{aligned}
\operatorname{Tr} & -\mathcal{R}^{3} \mathcal{R}_{5}+\mathcal{R}_{5} \mathcal{R}^{2} \mathcal{R}_{5}+\mathcal{R}^{2} \mathcal{R}_{5}^{2}+\left(\mathcal{R} \mathcal{R}_{5}\right)^{2} \\
& \left.-\frac{4}{3}\left(\mathcal{R}_{5}^{2} \mathcal{R} \mathcal{R}_{5}+\mathcal{R}_{5} \mathcal{R} \mathcal{R}_{5}^{2}+\mathcal{R} \mathcal{R}_{5}^{3}\right)\right] \rightarrow \frac{1}{p^{4}}\left(T_{4}+T_{4}^{\prime}\right)
\end{aligned}
$$

For the terms quartic in $M$ we obtain

$$
\begin{aligned}
T_{4}^{\prime}=-\frac{1}{4} F^{\prime}(M, \bar{M}, M, \bar{M})= & -\frac{4 i}{3} \operatorname{Tr}\left(\widetilde{M}^{\mu \nu} \bar{M}^{\rho \sigma} M_{\mu \nu} \bar{M}_{\rho \sigma}-M^{\mu \nu} \bar{M}^{\rho \sigma} M_{\mu \nu} \widetilde{\bar{M}}_{\rho \sigma}\right) \\
& -2 i \operatorname{Tr}\left(m \bar{M}_{\mu \nu} m \widetilde{\bar{M}}^{\mu \nu}-\bar{m} M_{\mu \nu} \bar{m}^{\mu \nu}\right),
\end{aligned}
$$


and for the terms quadratic in $M$, we find:

$$
\begin{aligned}
\operatorname{Tr} \mathcal{R}^{3} \mathcal{R}_{5} \rightarrow & 0, \quad \operatorname{Tr}\left(\mathcal{R} \mathcal{R}_{5}\right)^{2} \rightarrow-\frac{1}{p^{8}} H(M, \bar{M}) \\
\operatorname{Tr} \mathcal{R}^{2} \mathcal{R}_{5}^{2}= & \operatorname{Tr} \mathcal{R}_{5} \mathcal{R}^{2} \mathcal{R}_{5} \rightarrow \frac{1}{p^{8}} \frac{1}{2}[F(M, \bar{M})-F(\bar{M}, M)]=\frac{1}{p^{4}}\left(T_{4}^{\prime \prime}+T_{4}^{\prime \prime \prime}\right) \\
\operatorname{Tr} \mathcal{R} \mathcal{R}_{5}^{3}= & \operatorname{Tr} \mathcal{R}_{5} \mathcal{R}_{5}^{2}=\operatorname{Tr} \mathcal{R}_{5}^{2} \mathcal{R} \mathcal{R}_{5} \rightarrow \frac{1}{p^{8}} \frac{1}{2}\left[H\left(M, M_{I}\right)+H\left(\bar{M}, M_{I}\right)\right. \\
& \left.-F\left(M, M_{I}\right)-F\left(\bar{M}, M_{I}\right)+F\left(M_{I}, \bar{M}\right)+F\left(M_{I}, M\right)\right] \\
= & \frac{1}{p^{4}}\left(T_{4}^{\prime \prime}+T_{4}^{\prime \prime \prime}\right)-\frac{1}{p^{8}} \frac{1}{2} H(M, \bar{M})=-\frac{1}{2 p^{4}} T_{4} \\
T_{4}^{\prime \prime}= & \operatorname{Tr}\left(\left[\left\{\bar{m}, \widetilde{M}^{\mu \nu}\right\}-\left\{m, \widetilde{M}^{\mu \nu}\right\}\right]\left[\mathcal{J}_{\mu}, \mathcal{J}_{\nu}\right]\right) \\
T_{4}^{\prime \prime \prime}= & \frac{2 i}{3} \operatorname{Tr}\left[\left(\left\{M_{\mu \rho}, \widetilde{M}^{\mu \nu}\right\}-\left\{\bar{M}_{\mu \rho}, \widetilde{M}^{\mu \nu}\right\}\right)\left\{\mathcal{J}^{\rho}, \mathcal{J}_{\nu}\right\}\right]
\end{aligned}
$$

Then

$$
\begin{aligned}
T_{4} & =-2\left(T_{4}^{\prime \prime}+T_{4}^{\prime \prime \prime}\right)+\frac{1}{p^{4}} H(M, \bar{M}) \equiv-2 T_{4}^{\prime \prime}-t_{4} \\
& =-2 T_{4}^{\prime \prime}-\frac{8 i}{3} \operatorname{Tr}\left(\left\{\mathcal{J}^{\rho}, M_{\mu \rho}\right\}\left\{\mathcal{J}_{\nu}, \widetilde{M}^{\mu \nu}\right\}-\left\{\mathcal{J}^{\rho}, \bar{M}_{\mu \rho}\right\}\left\{\mathcal{J}_{\nu}, \bar{M}^{\mu \nu}\right\}\right) .
\end{aligned}
$$

To evaluate the cubic and quadratic terms, we use a shorthand notation according to which the covariant derivatives imply the matrix products:

$$
D_{\mu}^{ \pm} \mathcal{J}_{\nu} \equiv\left[D_{\mu}^{ \pm}, \mathcal{J}_{\nu}\right], \quad D_{\mu}^{ \pm} M \equiv D_{\mu}^{ \pm} M-M D_{\mu}^{\mp}
$$

where here $M$ is any mass matrix. Using the Dirac traces in (3.37), the first identity in (A.12), and the additional identities

$$
\begin{aligned}
\operatorname{Tr}([A, B] C) & =-\operatorname{Tr}(A[B, C]), D_{\mu}(M \bar{M})=\left[d_{\mu}^{+}, M \bar{M}\right], D_{\mu}(\bar{M} M)=\left[d_{\mu}^{-}, \bar{M} M\right], \\
{\left[d_{\mu}^{+}, M M_{I}\right] } & =\left(D_{\mu} M\right) M_{I}+M D_{\mu}^{-} M_{I}, \quad\left[d_{\mu}^{-}, \bar{M} M_{I}\right]=\left(D_{\mu} \bar{M}\right) M_{I}+\bar{M} D_{\mu}^{+} M_{I}, \\
\operatorname{Tr}(\{A, B\} C D) & =\operatorname{Tr}(B\{A, C D\})=\operatorname{Tr}(B\{A, C\} D)-\operatorname{Tr}(B C[A, D]),
\end{aligned}
$$

together with the facts [see (3.37)] that $\operatorname{Tr}\left(\sigma \cdot A \gamma_{\mu} \sigma \cdot B \gamma_{\nu}\right)$ and $\operatorname{Tr}\left(\sigma \cdot A \gamma_{\mu} \sigma \cdot B \gamma_{\nu} \gamma_{5}\right)$ are symmetric in $\{\mu, \nu\}$, and that $\left[L_{\mu}, \mathcal{J}_{\nu}\right]=0$, we obtain

$$
\begin{aligned}
\operatorname{Tr} \mathcal{R}^{2} \mathcal{R}_{5} \rightarrow & \frac{1}{p^{4}} \operatorname{Tr}\left\{-2 i \tilde{X}_{-}^{\mu \nu}(M, \bar{M}) \tilde{D}_{\mu}^{+} \mathcal{J}_{\nu}-2 i \widetilde{X}_{-}^{\mu \nu}(\bar{M}, M) \tilde{D}_{\mu}^{-} \mathcal{J}_{\nu}-L(M, \bar{M})\right. \\
& +\left[\widetilde{X}_{-}^{\mu \nu}\left(M_{I}, \bar{M}\right)-\widetilde{X}_{-}^{\mu \nu}\left(M, M_{I}\right)+\widetilde{X}_{+}^{\mu \nu}\left(M, M_{I}\right)\right] \tilde{G}_{\mu \nu}^{+}
\end{aligned}
$$




$$
\begin{aligned}
+ & {\left[\tilde{X}_{-}^{\mu \nu}\left(M_{I}, M\right)-\tilde{X}_{-}^{\mu \nu}\left(\bar{M}, M_{I}\right)+\tilde{X}_{+}^{\mu \nu}\left(\bar{M}, M_{I}\right)\right] \tilde{G}_{\mu \nu}^{-} } \\
+ & \frac{4}{3}\left[X^{+}(M, \bar{M})+X^{-}(\bar{M}, M)\right]+2\left(\bar{m} M^{\mu \nu}-m \bar{M}^{\mu \nu}\right) \hat{L}_{\mu \nu} \\
& \left.-\hat{L}_{\mu \nu}\left[X_{-}^{\mu \nu}\left(M_{I}, M\right)+X_{-}^{\mu \nu}\left(M_{I}, \bar{M}\right)\right]\right\}, \\
\operatorname{Tr} \mathcal{R} \mathcal{R}_{5}^{2}+ & \operatorname{Tr} \mathcal{R}_{5} \mathcal{R R}_{5} \rightarrow \frac{1}{p^{4}} \operatorname{Tr}\left\{-4 i\left[\tilde{X}_{-}^{\mu \nu}\left(M_{I}, \bar{M}\right)-\tilde{X}_{-}^{\mu \nu}\left(M, M_{I}\right)\right] \tilde{D}_{\mu}^{+} \mathcal{J}_{\nu}\right. \\
& +4 i\left[\tilde{X}_{-}^{\mu \nu}\left(M_{I}, M\right)-\tilde{X}_{-}^{\mu \nu}\left(\bar{M}, M_{I}\right)\right] \tilde{D}_{\mu}^{-} \mathcal{J}_{\nu}-2 L\left(\bar{M}, M_{I}\right)+2 L\left(M_{I}, M\right) \\
& -\frac{8}{3}\left[X^{+}\left(M, M_{I}\right)+X^{-}\left(M_{I}, M\right)-X^{+}\left(M_{I}, \bar{M}\right)-X^{-}\left(\bar{M}, M_{I}\right)\right] \\
& \left.+\left[\tilde{X}_{+}^{\mu \nu}\left(M_{I}, M_{I}\right)-2 \tilde{X}_{-}^{\mu \nu}\left(M_{I}, M_{I}\right)\right]\left(\tilde{G}_{\mu \nu}^{+}-\tilde{G}_{\mu \nu}^{-}\right)\right\}, \\
\operatorname{Tr} \mathcal{R}_{5}^{3} \rightarrow & \frac{1}{p^{4}} \operatorname{Tr}\left\{6 i \tilde{X}_{-}^{\mu \nu}\left(M_{I}, M_{I}\right)\left(\tilde{D}_{\mu}^{+} \mathcal{J}_{\nu}+\tilde{D}_{\mu}^{-} \mathcal{J}_{\nu}\right)\right. \\
& \left.-4\left[X^{+}\left(M_{I}, M_{I}\right)+X^{-}\left(M_{I}, M_{I}\right)\right]+3 L\left(M_{I}, M_{I}\right)\right\},
\end{aligned}
$$

where

$$
\begin{aligned}
& X^{ \pm}\left(M_{1}, M_{2}\right)=\left(\tilde{D}_{\rho}^{ \pm} M_{1}^{\mu \rho} \widetilde{M}_{\mu \nu}^{2}-\tilde{D}_{\rho}^{ \pm} \widetilde{M}_{1}^{\mu \rho} M_{\mu \nu}^{2}+\widetilde{M}_{\mu \nu}^{1} \tilde{D}_{\rho}^{\mp} M_{2}^{\mu \rho}-M_{\mu \nu}^{1} \tilde{D}_{\rho}^{\mp} \widetilde{M}_{2}^{\mu \rho}\right) \mathcal{J}^{\nu}, \\
& X_{ \pm}^{\mu \nu}\left(M_{1}, M_{2}\right)=M_{1}^{\mu \nu} m_{2} \pm m_{1} M_{2}^{\mu \nu} \quad \tilde{X}_{ \pm}^{\mu \nu}=\frac{1}{2} \epsilon^{\mu \nu \rho \sigma} \tilde{X}_{\rho \sigma}^{ \pm} \\
& L\left(M_{1}, M_{2}\right)=2\left\{L_{\mu}, m_{1}\right\}\left\{\mathcal{J}_{\nu}, m_{2}\right\}+\frac{4}{3}\left\{L_{\mu}, M_{1}^{\mu \nu}\right\}\left\{\mathcal{J}_{\nu}, M_{\mu \nu}^{2}\right\} \\
& +\frac{8}{3}\left(\left\{L_{\mu}, M_{1}^{\mu \rho}\right\}\left\{\mathcal{J}^{\nu}, M_{\rho \nu}^{2}\right\}+\left\{L^{\nu}, M_{1}^{\mu \rho}\right\}\left\{\mathcal{J}_{\mu}, M_{\rho \nu}^{2}\right\}\right) \text {. }
\end{aligned}
$$

Again, the traces on the right are over internal indices only. Here and throughout the remainder of this section, $\tilde{G}_{\mu \nu}^{ \pm}$is understood as one fourth of the Dirac trace of $\left[\tilde{D}_{\mu}^{ \pm}, \tilde{D}_{\nu}^{ \pm}\right]$, and has no contribution from the spin connection, and the derivative operators $\tilde{D}_{\mu}$ are understood to operate only on the object to their immediate right. The expressions (4.62) can be simplified further using the relations

$$
\begin{aligned}
X^{\mu \nu}\left(\tilde{D}_{\mu}^{+} \mathcal{J}_{\nu}+\tilde{D}_{\mu}^{-} \mathcal{J}_{\nu}\right) & =\frac{i}{2} X^{\mu \nu}\left(\tilde{G}_{\mu \nu}^{+}-\tilde{G}_{\mu \nu}^{-}\right) \\
X^{\mu \nu}\left(\tilde{D}_{\mu}^{+} \mathcal{J}_{\nu}-\tilde{D}_{\mu}^{-} \mathcal{J}_{\nu}\right) & =-2 i X^{\mu \nu}\left[\mathcal{J}_{\mu}, \mathcal{J}_{\nu}\right] \\
\left\{\mathcal{J}_{\mu}, M\right\} & =\frac{i}{2}\left(\tilde{D}_{\mu}^{+} M-\tilde{D}_{\mu}^{-} M\right),
\end{aligned}
$$

that follow from the definitions (4.42) and (4.59). Defining

$$
\begin{aligned}
& X_{1}=\operatorname{Tr}\left[X^{+}(M, \bar{M})+X^{-}(\bar{M}, M)\right], \\
& X_{2}=\operatorname{Tr}\left[X^{+}\left(M_{I}, M_{I}\right)+X^{-}\left(M_{I}, M_{I}\right)\right]
\end{aligned}
$$




$$
\begin{aligned}
& =i \operatorname{Tr}\left(\tilde{D}^{+\sigma} \widetilde{M}_{\sigma \mu}^{I} \tilde{D}_{\rho}^{-} M_{I}^{\rho \mu}-\tilde{D}^{+\sigma} M_{\sigma \mu}^{I} \tilde{D}_{\rho}^{-} \widetilde{M}_{I}^{\rho \mu}\right) \\
X_{3} & =i \operatorname{Tr}\left[\tilde{D}^{\sigma} \widetilde{M}_{\sigma \mu} \tilde{D}_{\rho}^{-} M_{I}^{\rho \mu}-\tilde{D}^{\sigma} M_{\sigma \mu} \tilde{D}_{\rho}^{-} \widetilde{M}_{I}^{\rho \mu}\right. \\
& \left.-\tilde{D}^{+\sigma} \widetilde{M}_{\sigma \mu}^{I} \tilde{D}_{\rho} \bar{M}^{\rho \mu}+\tilde{D}^{+\sigma} M_{\sigma \mu}^{I} \tilde{D}_{\rho} \widetilde{\bar{M}}^{\rho \mu}\right] \\
X_{4} & =\operatorname{Tr}\left[X^{+}\left(M, M_{I}\right)+X^{-}\left(M_{I}, M\right)-X^{+}\left(M_{I}, \bar{M}\right)-X^{-}\left(\bar{M}, M_{I}\right)\right] \\
& =-X_{1}+X_{3}-i \operatorname{Tr}\left(\tilde{D}^{\sigma} M_{\sigma \mu} \tilde{D}_{\rho} \widetilde{\bar{M}}^{\rho \mu}-\tilde{D}^{\sigma} \bar{M}_{\sigma \mu} \tilde{D}_{\rho} \widetilde{M}^{\rho \mu}\right)
\end{aligned}
$$

where we dropped total derivatives, we obtain

$$
\begin{aligned}
T_{3}= & \operatorname{Tr}\left(\mathcal{R}^{2} \mathcal{R}_{5}-\mathcal{R} \mathcal{R}_{5}^{2}-\mathcal{R}_{5} \mathcal{R} \mathcal{R}_{5}+\frac{4}{3} \mathcal{R}_{5}^{3}\right) \rightarrow \frac{1}{p^{4}}\left(\frac{4}{3} X_{3}-\frac{8}{3} X_{2}+t_{4}+2 T_{4}^{\prime \prime}\right) \\
& -\frac{4 i}{3 p^{4}} \operatorname{Tr}\left(\tilde{D}^{\sigma} M_{\sigma \mu} \tilde{D}_{\rho} \widetilde{\bar{M}}^{\rho \mu}-\tilde{D}^{\sigma} \bar{M}_{\sigma \mu} \tilde{D}_{\rho} \widetilde{M}^{\rho \mu}\right) \\
& +\frac{1}{p^{4}} \operatorname{Tr}\left\{\tilde{X}_{-}^{\mu \nu}(M, \bar{M}) \tilde{G}_{\mu \nu}^{+}-\tilde{X}_{-}^{\mu \nu}(\bar{M}, M) \tilde{G}_{\mu \nu}^{-}+2\left(\bar{m} M^{\mu \nu}-m \bar{M}^{\mu \nu}\right)\right. \\
& -\left[X_{-}^{\mu \nu}\left(M_{I}, M\right)+X_{-}^{\mu \nu}\left(M_{I}, \bar{M}\right)\right] \hat{L}_{\mu \nu}+\tilde{X}_{+}^{\mu \nu}\left(M_{I}, M_{I}\right)\left(\tilde{G}_{\mu \nu}^{+}-\tilde{G}_{\mu \nu}^{-}\right) \\
& \left.-\left[\tilde{X}_{+}^{\mu \nu}\left(M, M_{I}\right) \tilde{G}_{\mu \nu}^{+}+\tilde{X}_{+}^{\mu \nu}\left(\bar{M}, M_{I}\right) \tilde{G}_{\mu \nu}^{-}\right]-L(M, \bar{M})\right\}
\end{aligned}
$$

where $t_{4}, T_{4}^{\prime \prime}$ are defined in (4.57-58), and

$$
t_{4}=\frac{4}{3} X_{4}-\frac{8}{3} X_{2} .
$$

Finally, to obtain the logarithmically divergent parts of $\operatorname{Tr} \mathcal{R} \mathcal{R}_{5}$ and $\operatorname{Tr} \mathcal{R}_{5}^{2}$, we use (4.4749), giving

$$
\begin{aligned}
\operatorname{Tr} \mathcal{R}_{5}^{2} \rightarrow & \frac{8}{3 p^{4}} X_{2}-\frac{2}{p^{4}} L\left(M_{I}, M_{I}\right)+\frac{1}{p^{4}} \tilde{X}_{+}^{\mu \nu}\left(M_{I}, M_{I}\right)\left(\tilde{G}_{\mu \nu}^{+}-\tilde{G}_{\mu \nu}^{-}\right) \\
\operatorname{Tr} \mathcal{R} \mathcal{R}_{5} \rightarrow & \frac{4}{3 p^{4}} X_{3}+\frac{4 i}{3 p^{4}} \operatorname{Tr}\left(\left\{L^{\sigma}, M_{\sigma \mu}\right\}\left\{L_{\rho}, \overline{\bar{M}}^{\rho \mu}\right\}-\left\{L^{\sigma}, \bar{M}_{\sigma \mu}\right\}\left\{L_{\rho}, \widetilde{M}^{\rho \mu}\right\}\right) \\
& -\frac{1}{p^{4}} \operatorname{Tr}\left[i\left(\left\{L^{\rho}, m\right\} \tilde{D}_{\rho} \bar{m}-\tilde{D}^{\rho} m\left\{L_{\rho}, \bar{m}\right\}\right)+L(M, \bar{M})+2 L\left(M_{I}, M_{I}\right)\right] \\
& -\frac{2 i}{3 p^{4}} \operatorname{Tr}\left(\left\{L^{\rho}, M_{\sigma \mu}\right\} \tilde{D}_{\rho} \bar{M}^{\sigma \mu}-\tilde{D}^{\rho} M_{\sigma \mu}\left\{L_{\rho}, \bar{M}^{\sigma \mu}\right\}\right) \\
& +\frac{8 i}{3 p^{4}} \operatorname{Tr}\left(\left\{L^{\sigma}, M_{\sigma \mu}\right\} \tilde{D}_{\rho} \bar{M}^{\rho \mu}-\tilde{D}^{\sigma} M_{\sigma \mu}\left\{L_{\rho}, \bar{M}^{\rho \mu}\right\}\right) \\
& -\frac{4 i}{3 p^{4}} \operatorname{Tr}\left(\hat{L}_{\sigma}^{\rho}\left\{M^{\sigma \mu}, \bar{M}_{\rho \mu}\right\}\right)-\frac{1}{p^{4}} \operatorname{Tr}\left(\hat{L}_{\mu \nu}\left[X_{-}^{\mu \nu}\left(M, M_{I}\right)+X_{-}^{\mu \nu}\left(\bar{M}, M_{I}\right)\right]\right) \\
& +\frac{1}{p^{4}} \operatorname{Tr}\left[\tilde{X}_{+}^{\mu \nu}\left(M, M_{I}\right) \tilde{G}_{\mu \nu}^{+}+\tilde{X}_{+}^{\mu \nu}\left(\bar{M}, M_{I}\right) \tilde{G}_{\mu \nu}^{-}\right] \\
& +\frac{i}{p^{4}} r_{\nu}^{\mu} \operatorname{Tr}\left(\widetilde{M}^{\nu \rho} \bar{M}_{\mu \rho}-M^{\nu \rho} \widetilde{\bar{M}}_{\mu \rho}\right)+\text { total derivative. }
\end{aligned}
$$


Inserting these results in (4.39) gives

$$
\begin{aligned}
-\frac{i}{2} T_{-}= & g^{\frac{1}{2}} \frac{\ln \Lambda^{2}}{32 \pi^{2}}\left(T_{4}^{\prime}+T_{4}+T_{3}-\operatorname{Tr} \mathcal{R} \mathcal{R}_{5}+\operatorname{Tr} \mathcal{R}_{5}^{2}\right) \\
= & g^{\frac{1}{2}} \frac{\ln \Lambda^{2}}{32 \pi^{2}} \operatorname{Tr}\left\{T_{4}^{\prime}+\left[\tilde{X}_{-}^{\mu \nu}(M, \bar{M}) \tilde{G}_{\mu \nu}^{+}-\tilde{X}_{-}^{\mu \nu}(\bar{M}, M) \tilde{G}_{\mu \nu}^{-}\right]\right. \\
& -\frac{4 i}{3}\left(\tilde{D}^{\sigma} M_{\sigma \mu} \tilde{D}_{\rho} \overline{\bar{M}}^{\rho \mu}-\tilde{D}^{\sigma} \bar{M}_{\sigma \mu} \tilde{D}_{\rho} \widetilde{M}^{\rho \mu}\right) \\
& -i r_{\nu}^{\mu}\left(\widetilde{M}^{\nu \rho} \bar{M}_{\mu \rho}-M^{\nu \rho} \widetilde{M}_{\mu \rho}\right) \\
& +\left[\hat{L}_{\mu \nu}, \bar{m}^{\mu}\right] M^{\mu \nu}-\left[\hat{L}_{\mu \nu}, m\right] \bar{M}^{\mu \nu}+i\left(\left\{L^{\rho}, m\right\} \tilde{D}_{\rho} \bar{m}^{\prime}-\tilde{D}^{\rho} m\left\{L_{\rho}, \bar{m}\right\}\right) \\
& -\frac{4 i}{3}\left(\left\{L^{\sigma}, M_{\sigma \mu}\right\}\left\{L_{\rho}, \widetilde{M}^{\rho \mu}\right\}-\left\{L^{\sigma}, \bar{M}_{\sigma \mu}\right\}\left\{L_{\rho}, \widetilde{M}^{\rho \mu}\right\}\right) \\
& -\frac{8 i}{3}\left(\left\{L^{\sigma}, M_{\sigma \mu}\right\} \tilde{D}_{\rho} \bar{M}^{\rho \mu}-\tilde{D}^{\sigma} M_{\sigma \mu}\left\{L_{\rho}, \bar{M}^{\rho \mu}\right\}\right) \\
& +\frac{2 i}{3}\left[L^{\rho}\left(\left\{M_{\sigma \mu}, \tilde{D}_{\rho} \bar{M}^{\sigma \mu}\right\}-\left\{\tilde{D}_{\rho} M^{\sigma \mu}, \bar{M}_{\sigma \mu}\right\}\right)\right. \\
& \left.\left.+2 \hat{L}_{\mu \nu}\left\{M^{\mu \rho}, \bar{M}_{\rho}^{\nu}\right\}\right]\right\} .
\end{aligned}
$$

To evaluate (4.68), we note that the connection is block diagonal in the $\chi-\lambda-\alpha$ sector, and the axial part is diagonal in the $\lambda$ and $\alpha$ sectors, with $\mathcal{J}_{\lambda \lambda}=-\mathcal{J}_{\alpha \alpha}$. Using the reality and symmetry properties of the off-diagonal $\lambda$ - $\alpha$ masses:

$$
m_{\lambda \alpha}=-\bar{m}_{\lambda \alpha}=m_{\lambda \alpha}^{T}, \quad M_{\lambda \alpha}^{\mu \nu}=\bar{M}_{\lambda \alpha}^{\mu \nu}=-\left(M_{\lambda \alpha}^{\mu \nu}\right)^{T}
$$

it is easy to see that there is no contribution that involves only these masses. For the off-diagonal $\lambda$ - $\chi$ masses:

$$
\begin{aligned}
& m_{\lambda \chi}=m_{\lambda \chi}^{T}, \quad \widetilde{M}_{\lambda \chi}^{\mu \nu}=i M_{\lambda \chi}^{\mu \nu}, \quad \widetilde{\bar{M}}_{\lambda \chi}^{\mu \nu}=-i \bar{M}_{\lambda \chi}^{\mu \nu}, \quad M_{\lambda \chi}^{\mu \nu}=-\left(M_{\lambda \chi}^{\mu \nu}\right)^{T}, \\
& M_{\lambda \chi}^{\mu \nu} \bar{M}_{\mu \nu}^{\lambda \chi}=\bar{M}_{\lambda \chi}^{\mu \nu} \bar{M}_{\mu \nu}^{\lambda \chi}=0, \quad\left(M^{\mu \nu} \bar{M}_{\rho \nu}\right)_{a}^{a}=\left(M_{\rho \nu} \bar{M}^{\mu \nu}\right)_{a}^{a} .
\end{aligned}
$$

It follows from these relations that the last line in (4.68) vanishes.

Using the fermion matrix elements given in Appendix B.2, we obtain the nonvanishing contributions to $T_{-}$. The contributions to the mixed chiral-gauge sector are listed in Appendix B.5. All other contributions maybe found in Appendix C of II. Note that these expressions are fully covariant, although the expansion (4.49) of $T_{-}$is not. This noncovariance is necessarily the case since $T_{-}$contains the chiral anomaly that breaks classical Kähler invariance. However, the logarithmically divergent contributions are Kähler invariant, up to a possible dependence of the effective cut-off on the Kähler potential $[44,29,45]$. 
The ghostino determinant also contains helicity-odd contributions, but since it has the same form [30] as that of a four-component scalar, its evaluation is straightforward; the result is given in Appendix B.

\subsection{The One-Loop Effective Action}

The quantum action obtained by the prescriptions [30,31] defined in I described in Section 4.2 above takes the form

$$
\mathcal{L}_{q}=-\frac{1}{2} \Phi^{T} Z_{\Phi}\left(\hat{D}^{2}+H_{\Phi}\right) \Phi+\frac{1}{2} \bar{\Theta} Z_{\Theta}\left(i \not D-M_{\Theta}\right) \Theta+\mathcal{L}_{g h}+\mathcal{L}_{G h}
$$

The last two terms are the ghost and ghostino terms, respectively, $\Phi=\left(h_{\mu \nu}, \hat{\mathcal{A}}^{a}, \hat{z}^{i}, \hat{z}^{\bar{m}}\right)$ is a $2 N+4 N_{G}+10$ component scalar, $\Theta=\left(\psi_{\mu}, \lambda^{a}, \chi^{I}=L \chi^{i}+R \chi^{\bar{\imath}}, \alpha\right)$ is an $N+$ $N_{G}+5$ component Majorana fermion, where $N$ is the number of chiral multiplets, $N_{G}$ is the number of gauge multiplets, and the matrix valued metrics $Z_{\Phi}$ and $Z_{\Theta}$ are defined in Appendices B.1 and B.2 below. We set background fermion fields to zero, so $\psi_{\mu}, \lambda^{a}, \chi^{I}$ are the quantum gravitino, gaugino and chiral fermions, respectively, and $\alpha$ is the auxiliary field introduced to implement the gravitino gauge fixing condition. The matrix-valued covariant derivative $D_{\mu}$ is defined as in Section 3.2, and $\hat{D}_{\mu}$ includes additional terms in the connections that are given in $(4.26,32)$ above.

The one-loop contribution to the effective action is

$$
\begin{aligned}
\mathcal{L}_{1}= & \frac{i}{2} \operatorname{Tr} \ln \left(\hat{D}^{2}+H_{\Phi}\right)-\frac{i}{2} \operatorname{Tr} \ln \left(-i \not D+M_{\Theta}\right) \\
& +i \operatorname{Tr} \ln \left(D^{2}+H_{G h}\right)-i \operatorname{Tr} \ln \left(\hat{D}^{2}+H_{g h}\right) .
\end{aligned}
$$

The general results obtained in [48], [40], [37], [55] give for the bosonic determinant:

$$
\begin{aligned}
\frac{i}{2} \operatorname{Tr} & \ln \left(\hat{D}^{2}+H_{\Phi}\right)=\sqrt{g}\left\{\frac{\Lambda^{2}}{32 \pi^{2}} \operatorname{Tr}\left(\frac{1}{6} r-H_{\Phi}\right)\right. \\
& \left.+\frac{\ln \Lambda^{2}}{32 \pi^{2}} \operatorname{Tr}\left(\frac{1}{2} H_{\Phi}^{2}-\frac{1}{6} r H_{\Phi}+\frac{1}{12} \hat{G}_{\mu \nu}^{\Phi} \hat{G}_{\Phi}^{\mu \nu}+\frac{1}{120}\left[r^{2}+2 r^{\mu \nu} r_{\mu \nu}\right]\right)\right\}
\end{aligned}
$$

and for the fermionic determinant we have

$$
-\frac{i}{2} \operatorname{Tr} \ln \left(-i \not D+M_{\Theta}\right)=-\frac{i}{2}\left(T_{+}+T_{-}\right)=-\frac{i}{8} \operatorname{Tr} \ln \left[\hat{D}^{2}+H_{\Theta}\right]-\frac{i}{2} T_{-},
$$

where in (4.74) $\hat{D}_{\mu}$ and $H_{\Theta}$ are the $8 \times 8$ matrices defined in (4.29-32). The helicityaveraged part, $T_{+}$, of the fermion trace is $-\frac{1}{4}$ times (4.73) with the substitutions $H_{\Phi} \rightarrow$ 
$H_{\Theta}, \hat{G}_{\mu \nu}^{\Phi} \rightarrow \hat{G}_{\mu \nu}^{\ominus}$ and the trace includes a trace over Dirac indices, so

$$
\frac{1}{4}(\operatorname{Tr} 1)_{\Theta}=(\operatorname{Tr} 1)_{\Phi}-2 N_{G}=2 N+2 N_{G}+10
$$

Similarly, the ghost and ghostino contributions are equivalent to, respectively, -2 times the contribution of a $\left(4+N_{G}\right)$-component scalar and +2 times the contribution of a four-component scalar. For bosons, $H_{\Phi}$ and $\hat{D}_{\mu}$ are defined in Section 4.2 ; the matrix elements of $H$ and of

$$
\hat{G}_{\mu \nu}=\left[\hat{D}_{\mu}, \hat{D}_{\nu}\right]
$$

are given in Appendix B, and the helicity-odd contribution, $T_{-}$, of the fermion determinant that was evaluated in Section 4.3, eq. (4.40). The traces in (4.73-4.74) are given explicitly in Appendix C of II and in Appendix B of I. Here (see Appendix B.5) we list only the contributions to the matter and YM sector, involving background Yang-Mills fields and/or integration over the quantum Yang-Mills supermultiplet that were omitted in $\mathrm{I}$.

If $\mathcal{L}(g, K)$ is the standard Lagrangian $[42,13]$ for $N=1$ supergravity coupled to matter with space-time metric $g_{\mu \nu}$, Kähler potential $K$, and gauge kinetic normalization function $f_{a b}=\delta_{a b}(x+i y)$, then the logarithmically divergent part of the one-loop corrected Lagrangian is

$$
\mathcal{L}_{e f f}=\mathcal{L}\left(g_{R}, K_{R}\right)+\mathcal{L}_{0}+\frac{\ln \Lambda^{2}}{32 \pi^{2}}\left(X^{A B} \mathcal{L}_{A} \mathcal{L}_{B}+X^{A} \mathcal{L}_{A}\right)+\sqrt{g} \frac{\ln \Lambda^{2}}{32 \pi^{2}}\left(L+N_{G} L_{g}\right)
$$

where the classical Lagrangian $\mathcal{L}(g, K)$ is given in eq. (3.9) and also in Appendix B below, $\mathcal{L}_{0}$ is the one loop correction found in I after renormalization of $g, K$ [eq.(3.6) of I], and

$$
\begin{aligned}
L= & {\left[\mathcal{W}^{a b}\left(3 C_{G} \delta_{a b}-D_{i}\left(T_{b} z\right)^{j} D_{j}\left(T_{a} z\right)^{i}\right)+\text { h.c. }\right]-24 e^{-K} a \bar{a} \mathcal{D} } \\
& +\frac{N+5}{6}\left[\left(\mathcal{W}^{a b}+\overline{\mathcal{W}}^{a b}\right) \mathcal{D}_{a} \mathcal{D}_{b}-x\left(F_{\rho \mu}^{a}-i \tilde{F}_{\rho \mu}^{a}\right)\left(F_{a}^{\rho \nu}+i \tilde{F}_{a}^{\rho \nu}\right) \mathcal{D}_{\nu} z^{i} \mathcal{D}^{\mu} \bar{z}^{\bar{m}} K_{i \bar{m}}\right] \\
& +\frac{N+5}{3}\left[x^{2} \mathcal{W}_{a b} \overline{\mathcal{W}}^{a b}+2 \mathcal{D}^{2}-\mathcal{D}\left(K_{i \bar{m}} \mathcal{D}_{\rho} z^{i} \mathcal{D}^{\rho} \bar{z}^{\bar{m}}+2 \hat{V}+4 \mathcal{D} M_{\psi}^{2}\right)\right] \\
& +14 x^{2} \mathcal{W}_{a b} \overline{\mathcal{W}}^{a b}+12\left(\mathcal{W}^{a b}+\overline{\mathcal{W}}^{a b}\right) \mathcal{D}_{a} \mathcal{D}_{b}+22 \mathcal{D}^{2}+2 \mathcal{D}\left(11 \hat{V}+8 K_{i \bar{m}} \mathcal{D}_{\rho} z^{i} \mathcal{D}^{\rho} \bar{z}^{\bar{m}}\right) \\
& +x(\mathcal{W}+\overline{\mathcal{W}})\left(K_{i \bar{m}} \mathcal{D}_{\rho} z^{i} \mathcal{D}^{\rho} \bar{z}^{\bar{m}}-2 M_{\lambda}^{2}-2 V\right)+4 \mathcal{D}\left(27 M_{\psi}^{2}+7 M_{\lambda}^{2}\right) \\
& -26 i \mathcal{D}_{\mu} z^{j} \mathcal{D}_{\nu} \bar{z}^{\bar{m}} K_{i \bar{m}} \mathcal{D}^{a} F_{a}^{\mu \nu}+\frac{2}{x} \mathcal{D}_{\mu} z^{i} \mathcal{D}^{\mu} \bar{z}^{\bar{m}} R_{\bar{n} i \bar{m} j} \mathcal{D}_{a} D^{j}\left(T^{a} \bar{z}\right)^{\bar{n}} \\
& +\frac{2}{x} \mathcal{D}_{a} e^{-K^{*}} R_{n i}^{k j} A_{k} \bar{A}^{n} D_{j}\left(T^{a} z\right)^{i}+\frac{e^{-K}}{x} \mathcal{D}_{a}\left[\left(T^{a} z\right)^{i} R_{i}{ }^{j}{ }^{k} \bar{A}^{\ell} A_{j k}+\text { h.c. }\right]
\end{aligned}
$$




$$
\begin{aligned}
& +2 i F_{\mu \nu}^{a} D_{j}\left(T_{a} z\right)^{i} R_{i \bar{m} k}^{j} \mathcal{D}^{\mu} z^{k} \mathcal{D}^{\nu} \bar{z}^{\bar{m}}+\frac{4}{3} \mathcal{D} e^{-K} R_{j}^{i} A_{i} \bar{A}^{j}+\frac{4}{3} \mathcal{D} \mathcal{D}_{\mu} z^{i} \mathcal{D}^{\mu} \bar{z}^{\bar{m}} R_{i \bar{m}} \\
& +\frac{D_{i}\left(T_{a} z\right)^{i}}{6 x}\left[4 \mathcal{D}_{a}\left(\mathcal{D}_{\mu} z^{j} \mathcal{D}^{\mu} \bar{z}^{\bar{m}} K_{j \bar{m}}+\hat{V}+3 M_{\psi}^{2}-2 \mathcal{D}\right)+13 i F_{\mu \nu}^{a} K_{\bar{m} j} \mathcal{D}^{\mu} z^{j} \mathcal{D}^{\nu} \bar{z}^{\bar{m}}\right] \\
& +\frac{1}{2}\left(\frac{19}{x^{2}}+\rho^{i} \rho_{i}\right) \mathcal{D}\left(\partial_{\mu} x \partial^{\mu} x+\partial_{\mu} y \partial^{\mu} y\right)-\left(\frac{11}{x^{2}}+3 \rho^{i} \rho_{i}\right) \partial_{\mu} y \partial_{\nu} x \mathcal{D}^{a} F_{a}^{\mu \nu} \\
& -\frac{1}{4 x}\left(1+3 x^{2} \rho^{i} \rho_{i}\right)\left(\partial_{\rho} x \partial^{\nu} x+\partial_{\rho} y \partial^{\nu} y\right)\left(F_{\mu \nu}^{a}+i \tilde{F}_{\mu \nu}^{a}\right)\left(F_{a}^{\mu \rho}-i \tilde{F}_{a}^{\mu \rho}\right) \\
& -5\left\{\left[\frac{i}{x}\left(F_{a}^{\nu \mu}-i \tilde{F}_{a}^{\nu \mu}\right)+\frac{g_{\nu \mu}}{x^{2}} \mathcal{D}^{a}\right]\left(\partial_{\nu} x+i \partial_{\nu} y\right) K_{i \bar{m}}\left(T^{a} z\right)^{i} \mathcal{D}_{\mu} \bar{z}^{\bar{m}}+\text { h.c. }\right\} \\
& -\rho^{i} \rho_{i}\left\{\left[i x\left(F_{a}^{\nu \mu}-i \tilde{F}_{a}^{\nu \mu}\right)+g_{\nu \mu} \mathcal{D}^{a}\right]\left(\partial_{\nu} x+i \partial_{\nu} y\right) K_{i \bar{m}}\left(T^{a} z\right)^{i} \mathcal{D}_{\mu} \bar{z}^{\bar{m}}+\text { h.c. }\right\} \\
& +2 i x^{2} \rho^{i} \rho_{i} \mathcal{D}_{\mu} z^{j} \mathcal{D}_{\nu} \bar{z}^{\bar{m}} K_{i \bar{m}} \mathcal{D}^{a} F_{a}^{\mu \nu}+2 x^{2} \rho^{i} \rho_{i} \mathcal{D}\left[8 M_{\psi}^{2}+M_{\lambda}^{2}+2 \hat{V}-2 e^{-K} a \bar{a}\right] \\
& -x^{2} \rho^{i} \rho_{i}\left[2 x^{2} \mathcal{W}_{a b} \overline{\mathcal{W}}^{a b}\left(1-x^{2} \rho^{i} \rho_{i}\right)-4 x(\mathcal{W}+\overline{\mathcal{W}}) \mathcal{D}\right. \\
& \left.+\left(\mathcal{W}^{a b}+\overline{\mathcal{W}}^{a b}\right) \mathcal{D}_{a} \mathcal{D}_{b}+2 \mathcal{D}^{2}\right]+2 x^{2} \rho_{i} \rho^{i} \mathcal{D} \mathcal{D}_{\mu} z^{i} \mathcal{D}^{\mu} \bar{z}^{\bar{m}} K_{i \bar{m}} \\
& +\frac{x^{3}}{2} \rho_{i} \rho^{i}\left(F_{\rho \mu}^{a}-i \tilde{F}_{\rho \mu}^{a}\right)\left(F_{a}^{\rho \nu}+i \tilde{F}_{a}^{\rho \nu}\right) \mathcal{D}_{\nu} z^{i} \mathcal{D}^{\mu} \bar{z}^{\bar{m}} K_{i \bar{m}} \\
& +2 x\left[4 \rho_{i j}\left(T^{a} z\right)^{i}\left(T^{b} z\right)^{j} \mathcal{W}_{a b}+i \mathcal{D}_{\nu} \bar{z}^{\bar{m}}\left(T^{a} z\right)^{i} \rho_{\bar{m} i j}\left(F_{a}^{\mu \nu}-i \tilde{F}_{a}^{\mu \nu}\right) \mathcal{D}_{\mu} z^{j}+\text { h.c. }\right] \\
& +\left\{\rho_{i j} \mathcal{D}^{\mu} z^{j}\left[\frac{2}{x}\left(\partial_{\mu} x-i \partial_{\mu} y\right)\left(T^{a} z\right)^{i} \mathcal{D}_{a}-\frac{\bar{f}^{i}}{2} \mathcal{W}\left(\partial_{\mu} x+i \partial_{\mu} y\right)\right]+\text { h.c. }\right\} \\
& +\left\{\overline{\mathcal{W}}\left[2 x^{3} \rho^{i} \rho_{i} M_{\lambda}^{2}+\bar{f}^{i} a_{i}(\bar{a}-\bar{A}) e^{-K}-x^{2} \rho^{i j}\left(A_{j i k} \bar{A}^{k}-A_{i j} \bar{A}\right) e^{-K}\right]+\text { h.c. }\right\} \\
& +\left\{\left(e^{-K} \bar{A}^{j} A^{\bar{m}}+\mathcal{D}_{\mu} z^{j} \mathcal{D}^{\mu} \bar{z}^{\bar{m}}\right)\left[4 \rho_{\bar{m} i j}\left(T^{a} z\right)^{i} \mathcal{D}_{a}-\left(\rho_{\bar{m} i j}+\frac{\bar{f}_{\bar{m}}}{x} \rho_{i j}\right) \bar{f}^{i} \mathcal{D}\right]+\text { h.c. }\right\} \\
& -\frac{i}{2} K_{i \bar{m}}\left[\mathcal{D}^{\nu} \bar{z}^{\bar{m}}\left(T_{a} z\right)^{i}-\mathcal{D}^{\nu} z^{i}\left(T_{a} \bar{z}\right)^{\bar{m}}\right]\left[\bar{f}^{i} \rho_{i j} \mathcal{D}^{\rho} z^{j}\left(F_{\rho \nu}^{a}-i \tilde{F}_{\rho \nu}^{a}\right)+\text { h.c. }\right] \\
& +\frac{\mathcal{D}_{a}}{2 x}\left[K_{k \bar{m}}\left(T^{a} z\right)^{k} \mathcal{D}^{\mu} \bar{z}^{\bar{m}}+\frac{i}{2}\left(\partial_{\nu} x+i \partial_{\nu} y\right)\left(F^{\nu \mu}-i \tilde{F}^{\nu \mu}\right)+\text { h.c. }\right] \\
& \times\left(\rho_{i j} \mathcal{D}_{\mu} z^{i} \bar{f}^{j}+\text { h.c. }\right) \\
& -\left[\mathcal{W}^{a b} \rho_{i j} f^{i}\left(T_{a} z\right)^{j} \mathcal{D}_{b}+x^{2} \mathcal{D}_{p} z^{i} \mathcal{D}^{\rho} z^{j}\left(2 \rho_{i j} \mathcal{W}-R_{\bar{n} i \bar{m} j} \rho^{\bar{m} \bar{n}} \overline{\mathcal{W}}\right)+\text { h.c. }\right] \\
& +2 x^{2} \rho_{i j} \rho_{\bar{m}}^{j} \mathcal{D} \mathcal{D}_{\rho} z^{i} \mathcal{D}^{\rho} \bar{z}^{\bar{m}}+x^{4} \rho_{i j} \rho^{i j} \mathcal{W} \overline{\mathcal{W}}
\end{aligned}
$$

$$
\begin{aligned}
L_{g}= & x^{6}\left(\rho^{i} \rho_{i}\right)^{2} \mathcal{W} \overline{\mathcal{W}}-2 M_{\lambda}^{4}+3 M_{\psi}^{4}-2 M_{\psi}^{2} M_{\lambda}^{2}+\hat{V}^{2}+\mathcal{D}^{2}+6 e^{-K} a \bar{a} M_{\psi}^{2} \\
& +2 \hat{V}\left(2 M_{\psi}^{2}-M_{\lambda}^{2}+e^{-K} a \bar{a}\right)-e^{-K}\left(\bar{a}^{i} A_{i}+\text { h.c. }\right)\left(\hat{V}+M_{\psi}^{2}\right) \\
& +e^{-2 K} a_{i} \bar{A}^{i} \bar{a}^{j} A_{j}-2 e^{-2 K}\left(\bar{a}^{i} A_{i} a \bar{A}+\text { h.c. }\right)+x^{2} \rho_{i j} \mathcal{D}_{\mu} z^{i} \mathcal{D}^{\mu} z^{j} \rho_{\bar{n} \bar{m}} \mathcal{D}_{\nu} \bar{z}^{\bar{m}} \mathcal{D}^{\nu} \bar{z}^{n} \\
& +e^{-K} \mathcal{D}_{\mu} z^{i} \mathcal{D}^{\mu} \bar{z}^{\bar{m}}\left[\left(a_{i}-A_{i}\right)\left(\bar{a}_{\bar{m}}-\bar{A}_{\bar{m}}\right)+x^{2} \rho_{i k} \bar{A}^{k} \rho_{\bar{m}}^{j} A_{j}+\frac{\bar{f}_{\bar{m}} f_{i}}{4 x^{2}} a \bar{a}\right]
\end{aligned}
$$




$$
\begin{aligned}
& +e^{-K}\left\{\mathcal { D } _ { \mu } z ^ { i } \mathcal { D } ^ { \mu } z ^ { j } \left[\left(a_{i}-A_{i}\right)\left(\frac{f_{j}}{2 x} \bar{a}-x \rho_{j n} \bar{A}^{n}\right)\right.\right. \\
& \left.\left.\quad-\frac{f_{j}}{2 x} a_{i} \bar{A}-f_{i}(a-A) \rho_{j k} \bar{A}^{k}\right]+ \text { h.c. }\right\} \\
& +\frac{e^{-K}}{2 x}\left\{\mathcal{D}_{\mu} z^{i} \mathcal{D}^{\mu} \bar{z}^{\bar{m}} \bar{f}_{\bar{m}}\left[2 \bar{a} a_{i}-x \rho_{i k}(a-A) \bar{A}^{k}\right]\right. \\
& \left.\quad+\frac{f_{i} f_{j}}{2 x} \mathcal{D}_{\mu} z^{i} \mathcal{D}^{\mu} z^{j} \bar{a}(2 a-A)+\text { h.c. }\right\} \\
& +x\left(\rho_{i j} \mathcal{D}_{\mu} z^{i} \mathcal{D}^{\mu} z^{j}+\text { h.c. }\right)\left(M_{\psi}^{2}-\hat{V}\right) \\
& +e^{-K}\left[x \rho_{i j} \mathcal{D}_{\mu} z^{i} \mathcal{D}^{\mu} z^{j}\left(a_{k} \bar{A}^{k}-2 \bar{A} a\right)+\text { h.c. }\right] \\
& +\frac{1}{16 x^{4}}\left|\left(\partial_{\mu} x+i \partial_{\mu} y\right)\left(\partial^{\mu} x+i \partial^{\mu} y\right)\right|^{2}-x^{3} \rho^{i} \rho_{i}(\mathcal{W}+\overline{\mathcal{W}})\left(M_{\psi}^{2}+\hat{V}\right) \\
& +x^{3} \rho^{k} \rho_{k}\left[\mathcal{W}\left(x \rho_{i j} \mathcal{D}_{\mu} z^{i} \mathcal{D}^{\mu} z^{j}+e^{-K} A_{i} \bar{a}^{i}-2 e^{-K} \bar{a} A\right)+\text { h.c. }\right] \\
& +\frac{1}{6} K_{i \bar{m}} K_{j \bar{n}}\left(4 \mathcal{D}_{\mu} z^{i} \mathcal{D}^{\mu} z^{j} \mathcal{D}_{\nu} \bar{z}^{\bar{m}} \mathcal{D}^{\nu} \bar{z}^{\bar{n}}+\mathcal{D}_{\mu} z^{i} \mathcal{D}^{\mu} \bar{z}^{\bar{n}} \mathcal{D}_{\nu} \bar{z}^{\bar{m}} \mathcal{D}^{\nu} z^{j}\right) \\
& -\frac{1}{3}\left(\mathcal{D}_{\mu} z^{i} \mathcal{D}^{\mu} \bar{z}^{\bar{m}} K_{i \bar{m}}\right)^{2}+x^{2} \mathcal{W}_{a b} \overline{\mathcal{W}}^{a b}+\frac{1}{2}\left(\mathcal{W}_{a b}+\overline{\mathcal{W}}_{a b}\right) \mathcal{D}^{a} \mathcal{D}^{b} \\
& -\frac{1}{3} V^{2}+\frac{1}{3} M_{\lambda}^{2}\left(\mathcal{D}_{\mu} z^{i} \mathcal{D}^{\mu} \bar{z}^{\bar{m}} K_{i \bar{m}}-2 V\right) \\
& -\left(\frac{\partial_{\mu} x \partial^{\nu} x+\partial_{\mu} y \partial^{\nu} y}{x^{2}}\right) K_{i \bar{m}} \mathcal{D}_{\nu} z^{i} \mathcal{D}^{\mu} \bar{z}^{\bar{m}} \\
& +\frac{1}{3} V \mathcal{D}_{\mu} z^{i} \mathcal{D}^{\mu} \bar{z}^{\bar{m}} K_{i \bar{m}}+\left(\frac{\partial_{\nu} x \partial^{\nu} x}{6 x^{2}}+\frac{\partial_{\nu} y \partial^{\nu} y}{6 x^{2}}\right)\left(2 \mathcal{D}_{\mu} z^{i} \mathcal{D}^{\mu} \bar{z}^{\bar{m}} K_{i \bar{m}}-V\right) \\
& +\left(F_{\rho \mu}^{a}+i \tilde{F}_{\rho \mu}^{a}\right)\left(F_{a}^{\rho \nu}-i \tilde{F}_{a}^{\rho \nu}\right)\left(\frac{\partial^{\mu} x \partial_{\nu} x+\partial^{\mu} y \partial_{\nu} y}{4 x}-\frac{x}{2} K_{i \bar{m}} \mathcal{D}_{\nu} z^{i} \mathcal{D}^{\mu} \bar{z}^{\bar{m}}\right)(4.7
\end{aligned}
$$

Our notation is defined in Appendix A below. Here $\mathcal{W}=\mathcal{W}_{a}^{a}$, where

$$
\mathcal{W}_{b}^{a}=\frac{1}{4}\left(F_{\mu \nu}^{a} F_{b}^{\mu \nu}-i F_{\mu \nu}^{a} \tilde{F}_{b}^{\mu \nu}\right)-\frac{1}{2 x^{2}} \mathcal{D}^{a} \mathcal{D}_{b}
$$

is the bosonic part of the $F$-component of the composite chiral supermultiplet constructed from the Yang-Mills chiral superfield $W^{a}(\theta)=\lambda_{L}^{a}+O(\theta)$. The renormalized Kähler potential is

$$
K_{R}=K+\frac{\ln \Lambda^{2}}{32 \pi^{2}}\left[e^{-K}\left(A_{i j} \bar{A}^{i j}-2 A_{i} \bar{A}^{i}-4 A \bar{A}\right)-4 \mathcal{K}_{a}^{a}-\left(12+4 x^{2} \rho_{i} \rho^{i}\right) \mathcal{D}\right],
$$

and the renormalized space-time metric is given by

$$
\begin{aligned}
g_{\mu \nu} & =(1-\epsilon) g_{\mu \nu}^{R}+\epsilon_{\mu \nu} \\
\epsilon & =\epsilon^{0}-\frac{\ln \Lambda^{2}}{32 \pi^{2}}\left[\frac{N_{G}}{6}(r+V)+\frac{55-N}{6} \mathcal{D}+2 x^{2} \rho_{i} \rho^{i} \mathcal{D}+\frac{2}{3 x} \mathcal{D}_{a} D_{i}\left(T^{a} z\right)^{i}+\frac{N_{G}}{3} M_{\lambda}^{2}\right], \\
\epsilon_{\mu \nu} & =\epsilon_{\mu \nu}^{0}+\frac{\ln \Lambda^{2}}{32 \pi^{2}} \frac{N_{G}}{2}\left(r_{\mu \nu}-\frac{1}{2} r g_{\mu \nu}\right)
\end{aligned}
$$


$\cdot[z \varepsilon]$ u!

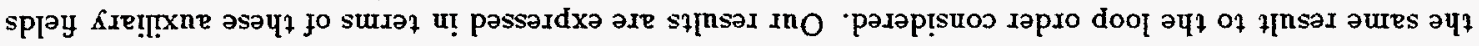

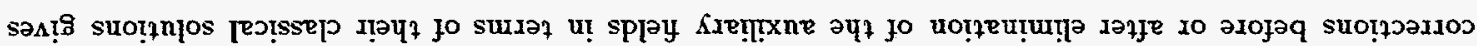

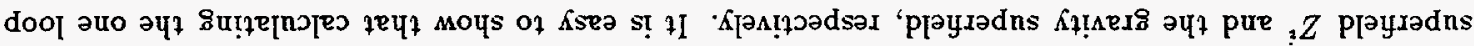

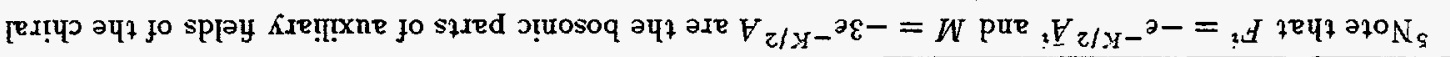

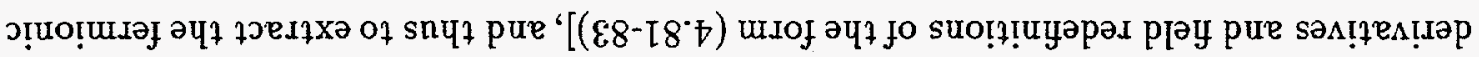

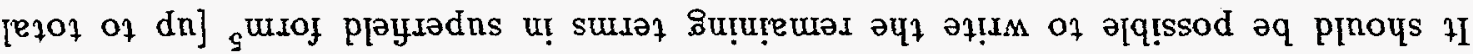
$\left(78^{\circ} \%\right)$

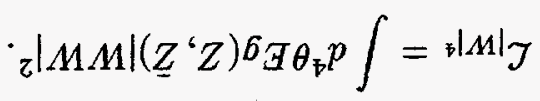

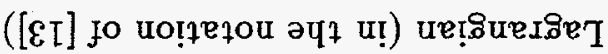

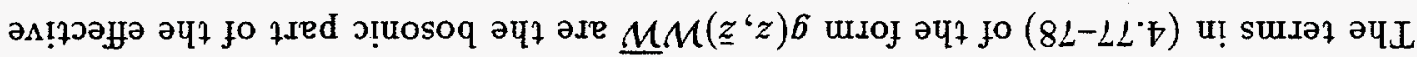

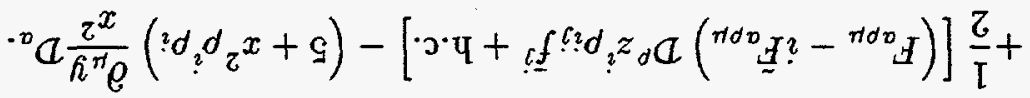

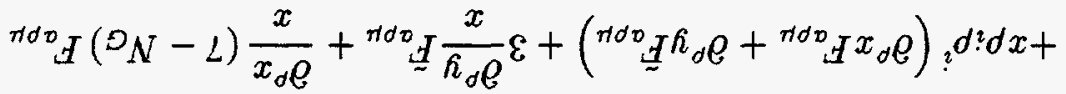

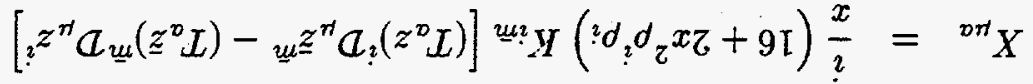

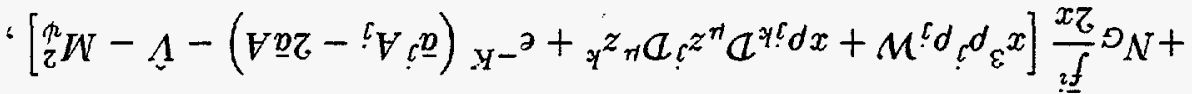

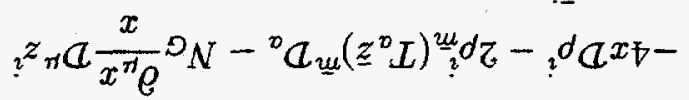

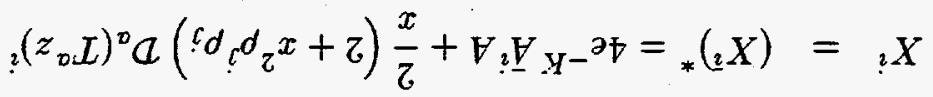

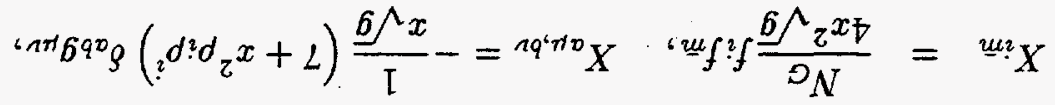

૫ฺ!!M

$\left(28^{\circ}\right)$

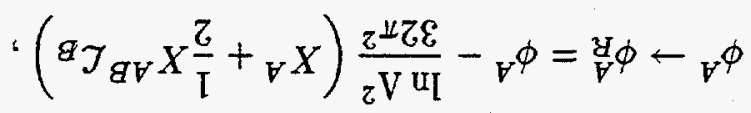

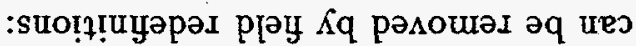

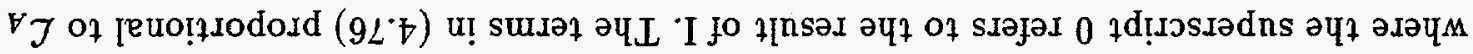

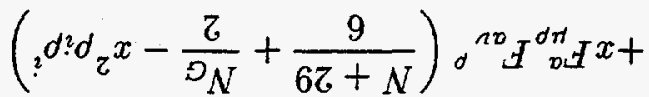

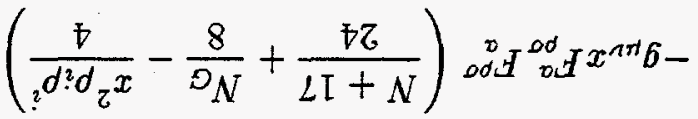

$$
\begin{aligned}
& \left(\underline{u}^{2} Y_{\underline{u}_{u}} \underline{z}_{n} a_{?} z^{n} a-\kappa_{d} \varrho \hat{\kappa}^{d} \varrho+x_{d} \varrho x^{d} \varrho\right) \frac{z^{x 9}}{J_{N}}{ }^{n} \sigma-
\end{aligned}
$$

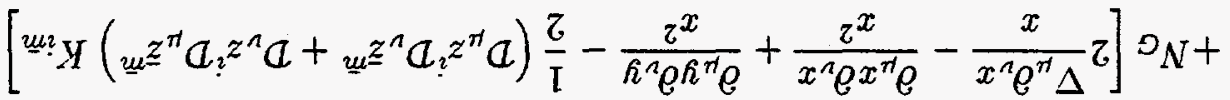


part of the Lagrangian for these higher dimension operators. However, there may be additional fermionic terms, e.g, those of the form [56]

$$
\mathcal{L}_{W^{2 n}}=\int d^{4} \theta E g(Z, \bar{Z})(W W)^{n>1}+\text { h.c. }
$$

that cannot be obtained in this way, as they have no purely bosonic components. The determination of such terms requires retaining fermionic background fields [57], [40], [49].

Notice that the coefficient of $\ln \Lambda^{2} F^{\mu \nu} F_{\mu \nu}$ is not a holomorphic function, except in the limits of a flat Kähler metric $\left(D_{i} \rightarrow \partial_{i}\right)$ and flat space-time $\left(M_{P l} \rightarrow \infty\right.$, in which case operators of dimension greater than four are suppressed). This nonholomorphicity is distinct from from the holomorphic anomaly $[25,58]$ that arises from the field-dependence of the infrared regulator masses. In other words, when the Kähler and/or space-time metric is not flat, there are corrections that correspond to D-terms as well as the usual F-terms.

The quadratically divergent contributions to the one-loop Lagrangian are given by (B.33-B.35). The Pauli-Villars regularization of these terms was given in [45]; they contribute additional renormalizations of the metric and the Kähler potential that are determined by the field-dependent squared masses of the Pauli-Villars regulator fields that play the role of effective cut-offs. The field dependence of the effective cut-offs in the logarithmically divergent contribution to the renormalized Kähler potential will generate additional terms in the effective Lagrangian proportional to

$$
D_{I} \ln \Lambda^{2}=2 \frac{D_{I} \Lambda}{\Lambda}, \quad I=i, \bar{\imath}
$$

that do not grow with the cut-off, and therefore have to be considered together with the finite terms that we have not evaluated here.

\subsection{The String Dilaton}

In effective supergravity from superstring theory, the classical Kähler potential $K(z, \bar{z})$, superpotential $W(z)$ and Yang-Mills normalization function $f_{a b}(z)$ take the forms

$$
\begin{aligned}
K(z, \bar{z}) & =-\ln (s+\bar{s})+G\left(y^{i}, \bar{y}^{\bar{m}}\right), \quad W(z)=W\left(y^{i}\right), \\
f_{a b}(z) & =\delta_{a b} k_{a} s, \quad y^{i}, \bar{y}^{\bar{m}} \neq s .
\end{aligned}
$$

Although we have restricted our analysis to the case $f_{a b}=\delta_{a b} f$, it is equally applicable to the case $f_{a b}=\delta_{a b} k_{a} f, k_{a}=$ constant, provided we make the substitutions $F_{\mu \nu}^{a} \rightarrow$ 
$k_{a}^{\frac{1}{2}} F_{\mu \nu}^{a}, A_{\mu}^{a} \rightarrow k_{a}^{\frac{1}{2}} A_{\mu}^{a}, T^{a} \rightarrow k_{a}^{-\frac{1}{2}} T^{a}, c_{a b c} \rightarrow k_{a}^{-\frac{1}{2}} c_{a b c},\left(c_{a b c} \neq 0\right.$ only if $\left.k_{a}=k_{b}=k_{c}\right)$ in all the relevant equations. Our results are therefore applicable to all known effective tree Lagrangians from superstrings, including those where the integers $k_{a} \geq 1$ correspond to higher affine levels [43]. In this case the operators $a, \rho_{i j}, 1-x^{2} \rho_{i} \rho^{i}$, and their covariant derivatives vanish identically. In particular $M_{\lambda}^{2}=M_{\psi}^{2} \equiv M^{2}$, and (4.76) reduces to

$$
\begin{aligned}
& \mathcal{L}_{e f f}=\mathcal{L}\left(g_{R}, K_{R}\right)+\mathcal{L}_{0}+\frac{\ln \Lambda^{2}}{32 \pi^{2}}\left(X^{A B} \mathcal{L}_{A} \mathcal{L}_{B}+X^{A} \mathcal{L}_{A}\right)+\sqrt{g} \frac{\ln \Lambda^{2}}{32 \pi^{2}}\left(L+N_{G} L_{g}\right), \\
& L=\left(\mathcal{W}^{a b}+\overline{\mathcal{W}}^{a b}\right)\left(3 C_{G} \delta_{a b}-D_{i}\left(T_{b} z\right)^{j} D_{j}\left(T_{a} z\right)^{i}\right)+2 \mathcal{D}\left(13 \hat{V}+9 K_{i \bar{m}} \mathcal{D}_{\mu} z^{i} \mathcal{D}^{\mu} \bar{z}^{\bar{m}}\right) \\
& +\frac{N+5}{12}\left[(s+\bar{s})^{2} \mathcal{W}_{a b} \overline{\mathcal{W}}^{a b}+2\left(\mathcal{W}^{a b}+\overline{\mathcal{W}}^{a b}\right) \mathcal{D}_{a} \mathcal{D}_{b}+8 \mathcal{D}^{2}-8\left(\hat{V}+2 M^{2}\right) \mathcal{D}\right] \\
& -\frac{N+5}{12}\left[(s+\bar{s})\left(F_{\rho \mu}^{a}-i \tilde{F}_{\rho \mu}^{a}\right)\left(F_{a}^{\rho \nu}+i \tilde{F}_{a}^{\rho \nu}\right)+4 g_{\mu}^{\nu} \mathcal{D}\right] \mathcal{D}_{\nu} z^{i} \mathcal{D}^{\mu} \bar{z}^{\bar{m}} K_{i \bar{m}} \\
& +\frac{7}{2}(s+\bar{s})^{2} \mathcal{W}_{a b} \overline{\mathcal{W}}^{a b}+11\left(\mathcal{W}^{a b}+\overline{\mathcal{W}}^{a b}\right) \mathcal{D}_{a} \mathcal{D}_{b}+20 \mathcal{D}^{2}+154 M^{2} \mathcal{D} \\
& +\frac{(s+\bar{s})}{2}(\mathcal{W}+\overline{\mathcal{W}})\left(K_{i \bar{m}} \mathcal{D}_{\rho} z^{i} \mathcal{D}^{\rho} \bar{z}^{\bar{m}}-2 \hat{V}+2 \mathcal{D}\right)-24 i \mathcal{D}_{\mu} z^{i} \mathcal{D}_{\nu} \bar{z}^{\bar{m}} K_{i \bar{m}} \mathcal{D}^{a} F_{a}^{\mu \nu} \\
& +\frac{(s+\bar{s})}{4}\left(F_{\rho \mu}^{a}-i \tilde{F}_{\rho \mu}^{a}\right)\left(F_{a}^{\rho \nu}+i \tilde{F}_{a}^{\rho \nu}\right) \mathcal{D}_{\nu} z^{i} \mathcal{D}^{\mu} \bar{z}^{\bar{m}} K_{i \bar{m}} \\
& +\frac{D_{i}\left(T_{a} z\right)^{i}}{3(s+\bar{s})}\left[4 \mathcal{D}^{a}\left(\hat{V}-2 \mathcal{D}+3 M^{2}+K_{j \bar{m}} \mathcal{D}_{\mu} z^{j} \mathcal{D}^{\mu} \bar{z}^{\bar{m}}\right)+13 i F_{\mu \nu}^{a} K_{\bar{m} j} \mathcal{D}^{\mu} z^{j} \mathcal{D}^{\nu} \bar{z}^{\bar{m}}\right] \\
& +\frac{D_{i}\left(T_{a} z\right)^{i}}{3(s+\bar{s})}\left[13 i F_{\mu \nu}^{a} K_{\bar{m} j} \mathcal{D}^{\mu} z^{j} \mathcal{D}^{\nu} \bar{z}^{\bar{m}}\right] \\
& -\left(\frac{4}{3} \mathcal{D} R_{i \bar{m}}+\frac{\mathcal{D}_{a}}{(s+\bar{s})} R_{\bar{n} i \bar{m} j} D^{j}\left(T^{a} \bar{z}\right)^{\bar{n}}\right)\left(e^{-K} \bar{A}^{i} A^{\bar{m}}+\mathcal{D}_{\mu} z^{i} \mathcal{D}^{\mu} \bar{z}^{\bar{m}}\right) \\
& +2 i F_{\mu \nu}^{a} D_{j}\left(T_{a} z\right)^{i} R_{i \bar{m} k}^{j} \mathcal{D}^{\mu} z^{k} \mathcal{D}^{\nu} \bar{z}^{\bar{m}}+\frac{2 e^{-K}}{(s+\bar{s})} \mathcal{D}_{a}\left[\left(T^{a} z\right)^{i} R_{i \ell}{ }^{j}{ }^{k} \bar{A}^{\ell} A_{j k}+\text { h.c. }\right] \\
& -\frac{12}{s+\bar{s}}\left\{\left[i \partial_{\nu} s\left(F_{a}^{\nu \mu}-i \tilde{F}_{a}^{\nu \mu}\right)+\frac{2 \partial^{\mu} s}{s+\bar{s}} \mathcal{D}_{a}\right] \mathcal{D}_{\mu} \bar{z}^{\bar{m}} K_{i \bar{m}}\left(T^{a} z\right)^{i}+\text { h.c. }\right\} \\
& -2 \frac{\partial_{\rho} s \partial^{\nu} \bar{s}}{(s+\bar{s})}\left(F_{\mu \nu}^{a}+i \tilde{F}_{\mu \nu}^{a}\right)\left(F_{a}^{\mu \rho}-i \tilde{F}_{a}^{\mu \rho}\right)+40 \frac{\partial_{\mu} s \partial^{\mu} \bar{s}}{(s+\bar{s})^{2}} \mathcal{D}+28 i \frac{\partial_{\mu} s \partial_{\nu} \bar{s}}{(s+\bar{s})^{2}} \mathcal{D}^{a} F_{a}^{\mu \nu}, \\
& L_{g}=\frac{(s+\bar{s})^{2}}{4}\left(\mathcal{W}_{a b} \overline{\mathcal{W}}^{a b}+\mathcal{W} \overline{\mathcal{W}}\right)-\frac{s+\bar{s}}{2}(\mathcal{W}+\overline{\mathcal{W}})\left(M^{2}+\hat{V}\right)+\mathcal{D}^{2} \\
& +\frac{1}{2}\left(\mathcal{W}_{a b}+\overline{\mathcal{W}}_{a b}\right) \mathcal{D}^{a} \mathcal{D}^{b}-2\left(\mathcal{D}+\frac{1}{3} V\right) M^{2}+\frac{1}{3}\left(M^{2}+V\right) \mathcal{D}_{\mu} z^{i} \mathcal{D}^{\mu} \bar{z}^{\bar{m}} K_{i \bar{m}} \\
& -\frac{1}{3} V^{2}+\frac{1}{6} K_{i \bar{m}} K_{j \bar{n}}\left(4 \mathcal{D}_{\mu} z^{i} \mathcal{D}^{\mu} z^{j} \mathcal{D}_{\nu} \bar{z}^{\bar{m}} \mathcal{D}^{\nu} \bar{z}^{\bar{n}}+\mathcal{D}_{\mu} z^{i} \mathcal{D}^{\mu} \bar{z}^{\bar{n}} \mathcal{D}_{\nu} \bar{z}^{\bar{m}} \mathcal{D}^{\nu} z^{j}\right) \\
& -\frac{1}{3} K_{i \bar{m}} K_{j \bar{n}} \mathcal{D}_{\mu} z^{i} \mathcal{D}^{\mu} \bar{z}^{\bar{m}} \mathcal{D}_{\nu} z^{j} \mathcal{D}^{\nu} \bar{z}^{\bar{n}}+\frac{2}{3}\left(2 K_{i \bar{m}} \mathcal{D}_{\mu} z^{i} \mathcal{D}^{\mu} \bar{z}^{\bar{m}}-V\right) \frac{\partial_{\nu} s \partial^{\nu} \bar{s}}{(s+\bar{s})^{2}} \\
& +\frac{\partial_{\mu} s \partial^{\mu} s \partial_{\nu} \bar{s} \partial^{\nu} \bar{s}}{(s+\bar{s})^{4}}-\frac{2 \partial_{\mu} s \partial_{\nu} \bar{s}}{(s+\bar{s})^{2}} K_{i \bar{m}}\left(\mathcal{D}^{\mu} z^{i} \mathcal{D}^{\nu} \bar{z}^{\bar{m}}+\mathcal{D}^{\mu} \bar{z}^{\bar{m}} \mathcal{D}^{\nu} z^{i}\right) \\
& +\left(F_{\rho \mu}^{a}+i \tilde{F}_{\rho \mu}^{a}\right)\left(F_{a}^{\rho \nu}-i \tilde{F}_{a}^{\rho \nu}\right)\left(\frac{\partial_{\nu} s \partial^{\mu} \bar{s}}{2(s+\bar{s})}-\frac{s+\bar{s}}{4} K_{i \bar{m}} \mathcal{D}_{\nu} z^{i} \mathcal{D}^{\mu} \bar{z}^{\bar{m}}\right),
\end{aligned}
$$


with, instead of (4.10),

$$
K_{R}=K+\frac{\ln \Lambda^{2}}{32 \pi^{2}}\left(e^{-K}\left[A_{i j} \bar{A}^{i j}-2 A_{i} \bar{A}^{i}+\left(N_{G}-4\right) A \bar{A}\right]-4 \mathcal{K}_{a}^{a}-16 \mathcal{D}\right) .
$$

Here we have considered only the standard chiral multiplet formulation of supergravity. Their is reason to believe [26], [29], [59] that the dilaton in the effective field theory from superstrings should be described, in fact, by a linear multiplet, which is dual to the chiral multiple used here. It has been shown [60] that a variety of classically dual theories remain equivalent at the quantum level. In [45] it was observed that once the ambiguous matrix elements (4.41) have been fixed in a supersymmetric way that admits Pauli-Villars regularization, the axion $y$ of the dilaton supermultiplet appears only through its dual $h^{\nu \rho \sigma}=\epsilon^{\nu \rho \sigma \mu} \partial_{\mu} y / 4 x^{2}$. This suggests that the properly regulated chiral supergravity theory also remains equivalent to the linear multiplet version for the dilaton at the quantum level. Some loop corrections using the linear multiple formulation have been carried out in [61].

Further simplifications occur in specific models, such as the untwisted sectors from orbifold compactifications where the scalar Riemann tensor is covariantly constant and the Ricci tensor is proportional to the Kähler metric for each untwisted sector.

\subsection{Here Is Where The Action Is.}

In this section we recapitulate the results of the calculations of paper II (as described in the previous chapters) put together with the results of I, and present the full one-loop bosonic effective action of supergravity plus YM and chiral matter:

$$
\begin{aligned}
\mathcal{L}_{e f f}= & \mathcal{L}(K)+\mathcal{L}_{1-\text { loop }}=\mathcal{L}\left(K_{R}\right)+\sqrt{g} \frac{\Lambda^{2}}{32 \pi^{2}} L_{0}+\sqrt{g} \frac{\ln \Lambda^{2}}{32 \pi^{2}}\left(L_{1}+L_{2}+N_{G} L_{g}\right), \\
L_{0}= & \frac{r}{2}\left(N_{G}-1\right)+2 K_{i \bar{m}}\left(\mathcal{D}_{\mu} z^{i} \mathcal{D}^{\mu} \bar{z}^{\bar{m}}+5 F^{i} \bar{F}^{\bar{m}}\right)-8 \mathcal{D}+\frac{28}{9} M \bar{M} \\
& +2 N\left(K_{i \bar{m}} F^{i} \bar{F}^{\bar{m}}-\frac{2}{9} M \bar{M}-\frac{r}{4}\right)+2 x^{-1} \mathcal{D}_{a} D_{i}\left(T^{a} z\right)^{i} \\
& -\left(N_{G} \frac{f_{i} \bar{f}_{\tilde{m}}}{2 x^{2}}+2 R_{i \bar{m}}\right)\left(F^{i} \bar{F}^{\bar{m}}+\mathcal{D}_{\mu} z^{i} \mathcal{D}^{\mu} \bar{z}^{\bar{m}}\right) \\
L_{1}= & \frac{N+5}{27}\left[K_{i \bar{m}}\left(2 F^{i} \bar{F}^{\bar{m}}-\mathcal{D}_{\mu} z^{i} \mathcal{D}^{\mu} \bar{z}^{\bar{m}}\right) M \bar{M}-\frac{1}{3}(M \bar{M})^{2}\right] \\
& +\frac{N+5}{3} K_{i \bar{m}} K_{j \bar{n}}\left(\mathcal{D}_{\mu} z^{j} \mathcal{D}^{\mu} \bar{z}^{\bar{m}} F^{j} \bar{F}^{\bar{n}}+\mathcal{D}_{\mu} z^{j} \mathcal{D}^{\mu} z^{i} \mathcal{D}_{\nu} \bar{z}^{\bar{m}} \mathcal{D}^{\nu} \bar{z}^{\bar{n}}\right) \\
& +8\left(K_{j \bar{n}} F^{j} \bar{F}^{\bar{n}}-\frac{1}{3} M \bar{M}\right)\left[K_{i \bar{m}}\left(F^{i} \bar{F}^{\bar{m}}+\mathcal{D}_{\mu} z^{i} \mathcal{D}^{\mu} \bar{z}^{\bar{m}}\right)-\frac{1}{3} M \bar{M}\right]
\end{aligned}
$$




$$
\begin{aligned}
& -4\left(\mathcal{D}_{\mu} \bar{z}^{\bar{m}} \mathcal{D}^{\mu} z^{i} K_{i \bar{m}}\right)^{2}-8 \mathcal{D}_{\mu} z^{j} \mathcal{D}^{\mu} z^{i} \mathcal{D}_{\nu} \bar{z}^{\bar{m}} \mathcal{D}^{\nu} \bar{z}^{\bar{n}} K_{i \bar{n}} K_{j \bar{m}} \\
& -\left(\mathcal{D}_{\mu} z^{i} \mathcal{D}^{\mu} \bar{z}^{\bar{m}}+F^{i} \bar{F}^{\bar{m}}\right)\left[\frac{2}{3} K_{i \bar{m}} R_{j \bar{n}}\left(\mathcal{D}_{\mu} z^{j} \mathcal{D}^{\mu} \bar{z}^{\bar{n}}+F^{j} \bar{F}^{\bar{n}}\right)+4 R_{\bar{n} i \bar{m} j} \bar{F}^{\bar{n}} F^{j}\right] \\
& +\frac{1}{3} \mathcal{D}_{\mu} z^{i} \mathcal{D}_{\nu} \bar{z}^{\bar{m}} K_{i \bar{m}}\left(R_{j \bar{n}}-2 K_{j \bar{n}}\right)\left(\mathcal{D}^{\mu} z^{j} \mathcal{D}^{\nu} \bar{z}^{\bar{n}}-\mathcal{D}^{\nu} z^{j} \mathcal{D}^{\mu} \bar{z}^{\bar{n}}\right) \\
& -\left\{\left(\mathcal{D}_{\mu} z^{i} \mathcal{D}^{\mu} \bar{z}^{\bar{m}}+F^{i} \bar{F}^{\bar{m}}\right)\left[e^{-K} R_{n i}^{j k} A_{j k} \bar{A}_{\bar{m}}^{n}+e^{-K / 2}\left(D_{\bar{m}} R_{n i}^{j k}\right) A_{j k} F^{n}\right]+\text { h.c. }\right\} \\
& -e^{-K / 2}\left[\frac{1}{3} R_{j i}^{k \ell} A_{k \ell}\left(\bar{M} \mathcal{D}_{\mu} z^{i} \mathcal{D}^{\mu} z^{j}+M F^{i} F^{j}\right)\right. \\
& \left.+\mathcal{D}_{\mu} z^{i} \mathcal{D}^{\mu} z^{j} F^{m}\left(R_{j m}^{k \ell} A_{i k \ell}-R_{j i}^{k \ell} A_{m k \ell}\right)+\text { h.c. }\right] \\
& +R^{k}{ }_{j}^{\ell}{ }_{i} R_{\bar{n} k \bar{m} \ell}\left(\mathcal{D}_{\mu} z^{j} \mathcal{D}^{\mu} z^{i} \mathcal{D}_{\nu} \bar{z}^{\bar{n}} \mathcal{D}^{\nu} \bar{z}^{\bar{m}}-F^{j} F^{i} \bar{F}^{\bar{n}} \bar{F}^{\bar{m}}\right) \\
& +R_{j \bar{m} i}^{k} R_{\ell \bar{n} k}^{i}\left(\mathcal{D}_{\mu} z^{j} \mathcal{D}^{\mu} \bar{z}^{\bar{m}} \mathcal{D}_{\nu} z^{\ell} \mathcal{D}^{\nu} \bar{z}^{\bar{n}}+2 \mathcal{D}_{\mu} z^{j} \mathcal{D}^{\mu} \bar{z}^{\bar{m}} F^{\ell} \bar{F}^{\bar{n}}+F^{j} \bar{F}^{\bar{m}} F^{\ell} \bar{F}^{\bar{n}}\right) \\
& +\mathcal{D}_{\mu} z^{j} \mathcal{D}_{\nu} \bar{z}^{\bar{m}} R_{i \bar{m} j}^{k} \mathcal{D}^{\mu} z^{\ell} \mathcal{D}^{\nu} \bar{z}^{\bar{n}} R_{k \bar{n} \ell}^{i}-\mathcal{D}_{\mu} z^{j} \mathcal{D}_{\nu} \bar{z}^{\bar{m}} R_{i \bar{m} j}^{k} \mathcal{D}^{\nu} z^{\ell} \mathcal{D}^{\mu} \bar{z}^{\bar{n}} R_{k \bar{n} \ell}^{i} \\
& L_{2}=\left[\mathcal{W}^{a b}\left(3 C_{G} \delta_{a b}-D_{i}\left(T_{b} z\right)^{j} D_{j}\left(T_{a} z\right)^{i}\right)+\text { h.c. }\right]+2 M \bar{M} \mathcal{D}+22 \mathcal{D}^{2} \\
& +\frac{N+5}{6}\left[2 \operatorname{Re} \mathcal{W}^{a b} \mathcal{D}_{a} \mathcal{D}_{b}-x\left(F_{\rho \mu}^{a}-i \tilde{F}_{\rho \mu}^{a}\right)\left(F_{a}^{\rho \nu}+i \tilde{F}_{a}^{\rho \nu}\right) \mathcal{D}_{\nu} z^{i} \mathcal{D}^{\mu} \bar{z}^{\bar{m}} K_{i \bar{m}}\right] \\
& +\frac{N+5}{3}\left[x^{2} \mathcal{W}_{a b} \overline{\mathcal{W}}^{a b}+2 \mathcal{D}^{2}-\mathcal{D} K_{i \bar{m}}\left(\mathcal{D}_{\rho} z^{i} \mathcal{D}^{\rho} \bar{z}^{\bar{m}}+2 F^{i} \bar{F}^{\bar{m}}\right)+\frac{2}{9} \mathcal{D} M \bar{M}\right] \\
& +24 \operatorname{Re} \mathcal{W}^{a b} \mathcal{D}_{a} \mathcal{D}_{b}+2 x \operatorname{Re} \mathcal{W}\left[\frac{2}{3} M \bar{M}+K_{i \bar{m}}\left(\mathcal{D}_{\rho} z^{i} \mathcal{D}^{\rho} \bar{z}^{\bar{m}}-2 F^{i} \bar{F}^{\bar{m}}\right)\right] \\
& -4 x \operatorname{Re} \mathcal{W}^{a b} \mathcal{D}+2 \mathcal{D} K_{i \bar{m}}\left(8 \mathcal{D}_{\rho} z^{i} \mathcal{D}^{\rho} \bar{z}^{\bar{m}}+11 F^{i} \bar{F}^{\bar{m}}\right)-26 i \mathcal{D}_{\mu} z^{j} \mathcal{D}_{\nu} \bar{z}^{\bar{m}} K_{i \bar{m}} \mathcal{D}^{a} F_{a}^{\mu \nu} \\
& +14 x^{2} \mathcal{W}_{a b} \overline{\mathcal{W}}^{a b}+\left(\mathcal{D}_{\mu} z^{i} \mathcal{D}^{\mu} \bar{z}^{\bar{m}}+F^{i} \bar{F}^{\bar{m}}\right)\left[\frac{2}{x} R_{\bar{n} i \bar{m} j} \mathcal{D}_{a} D^{j}\left(T^{a} \bar{z}\right)^{\bar{n}}+\frac{4}{3} \mathcal{D} R_{i \bar{m}}\right] \\
& +\frac{D_{i}\left(T_{a} z\right)^{i}}{6 x}\left\{4 \mathcal{D}_{a}\left[\left(\mathcal{D}_{\mu} z^{j} \mathcal{D}^{\mu} \bar{z}^{\bar{m}}+F^{j} \bar{F}^{\bar{m}}\right) K_{j \bar{m}}-2 \mathcal{D}\right]+13 i F_{\mu \nu}^{a} K_{\bar{m} j} \mathcal{D}^{\mu} z^{j} \mathcal{D}^{\nu} \bar{z}^{\bar{m}}\right] \\
& +2 i F_{\mu \nu}^{a} D_{j}\left(T_{a} z\right)^{i} R_{i \bar{m} k}^{j} \mathcal{D}^{\mu} z^{k} \mathcal{D}^{\nu} \bar{z}^{\bar{m}}-\frac{e^{-K / 2}}{x} \mathcal{D}_{a}\left[\left(T^{a} z\right)^{i} R_{i}{ }^{j}{ }^{k}{ }^{k} F^{\ell} A_{j k}+\text { h.c. }\right] \\
& +\frac{f_{i} \bar{f}_{\bar{m}}}{2 x^{2}}\left(F^{i} \bar{F}^{\bar{m}}+19 \mathcal{D}_{\mu} z^{i} \mathcal{D}^{\mu} \bar{z}^{\bar{m}}\right) \mathcal{D}-\mathcal{D}\left(4+\frac{f_{k} \bar{f}^{k}}{6 x^{2}}\right)\left(\frac{f_{i}}{x} F^{i} M+\text { h.c. }\right) \\
& +\frac{\bar{f}_{\bar{m}} f_{j}}{2 x} \bar{F}^{\bar{m}} F^{j}(\mathcal{W}+\overline{\mathcal{W}})+\frac{f_{k} \bar{f}^{k} \bar{f}_{\bar{m}} f_{j}}{8 x^{4}} \mathcal{D}\left(\mathcal{D}_{\mu} \bar{z}^{\bar{m}} \mathcal{D}^{\mu} z^{j}-\bar{F}^{\bar{m}} F^{j}\right) \\
& -\frac{f_{i} \bar{f}^{\bar{m}}}{x}\left[\frac{1}{4}\left(F_{\mu \nu}^{a}+i \tilde{F}_{\mu \nu}^{a}\right)\left(F_{a}^{\mu \rho}-i \tilde{F}_{a}^{\mu \rho}\right) \mathcal{D}_{\rho} z^{i} \mathcal{D}^{\nu} \bar{z}^{\bar{m}}+\frac{11 i}{2 x} \mathcal{D}_{\mu} z^{i} \mathcal{D}^{\nu} \bar{z}^{\bar{m}} \mathcal{D}^{a} F_{a}^{\mu \nu}\right] \\
& -\left(5+\frac{f_{k} \bar{f}^{k}}{4 x^{2}}\right)\left\{\left[\frac{i}{x}\left(F_{a}^{\nu \mu}-i \tilde{F}_{a}^{\nu \mu}\right)+\frac{g^{\nu \mu}}{x^{2}} \mathcal{D}^{a}\right] f_{j} \mathcal{D}_{\nu} z^{j} K_{i \bar{m}}\left(T^{a} z\right)^{i} \mathcal{D}_{\mu} \bar{z}^{\bar{m}}+\text { h.c. }\right\} \\
& -\frac{3 f_{k} \bar{f}^{k} f_{i} \tilde{f}_{\bar{m}}}{16 x^{4}}\left[x\left(F_{\mu \nu}^{a}+i \tilde{F}_{\mu \nu}^{a}\right)\left(F_{a}^{\mu \rho}-i \tilde{F}_{a}^{\mu \rho}\right) \mathcal{D}_{\rho} z^{i} \mathcal{D}^{\nu} \bar{z}^{\bar{m}}\right. \\
& \left.+2 i \mathcal{D}_{\mu} z^{i} \mathcal{D}^{\nu} \bar{z}^{\bar{m}} \mathcal{D}^{a} F_{a}^{\mu \nu}\right]
\end{aligned}
$$




$$
\begin{aligned}
& +\frac{\bar{f}^{i} f_{i}}{2 x^{2}} K_{i \bar{m}}\left[\mathcal{D}\left(\mathcal{D}_{\mu} z^{i} \mathcal{D}^{\mu} \bar{z}^{\bar{m}}+2 F^{i} \bar{F}^{\bar{m}}\right)+i \mathcal{D}_{\mu} z^{j} \mathcal{D}_{\nu} \bar{z}^{\bar{m}} \mathcal{D}^{a} F_{a}^{\mu \nu}\right] \\
& -\frac{\bar{f}^{i} f_{i}}{8 x^{2}}\left[8 x(\mathcal{W}+\overline{\mathcal{W}}) \mathcal{D}+\left(4 x^{2}-\bar{f}^{j} f_{j}\right) \mathcal{W}_{a b} \overline{\mathcal{W}}^{a b}+2\left(\mathcal{W}^{a b}+\overline{\mathcal{W}}^{a b}\right) \mathcal{D}_{a} \mathcal{D}_{b}\right] \\
& +\frac{f_{i} \bar{f}^{i}}{8 x^{2}}\left[x\left(F_{\rho \mu}^{a}-i \tilde{F}_{\rho \mu}^{a}\right)\left(F_{a}^{\rho \nu}+i \tilde{F}_{a}^{\rho \nu}\right) \mathcal{D}_{\nu} z^{i} \mathcal{D}^{\mu} \bar{z}^{\bar{m}} K_{i \bar{m}}-4 \mathcal{D}^{2}\right]+x^{4} \rho_{i j} \rho^{i j} \mathcal{W W} \\
& +\left\{\left(F^{j} \bar{F}^{\bar{m}}+\mathcal{D}_{\mu} z^{j} \mathcal{D}^{\mu} \bar{z}^{\bar{m}}\right)\left[4 \rho_{\bar{m} i j}\left(T^{a} z\right)^{i} \mathcal{D}_{a}-\left(\rho_{\bar{m} i j}+\frac{\bar{f}_{\bar{m}}}{x} \rho_{i j}\right) \bar{f}^{i} \mathcal{D}\right]+\text { h.c. }\right\} \\
& +e^{-K / 2}\left\{\overline{\mathcal{W}}\left[x^{2} \rho^{i j}\left(A_{j i k} F^{k}-\frac{1}{3} A_{i j} \bar{M}\right)+\frac{f_{k} \bar{f}^{i} \bar{f}^{j}}{4 x^{2}} F^{k} A_{i j}\right]+\text { h.c. }\right\} \\
& +2 x\left[4 \rho_{i j}\left(T^{a} z\right)^{i}\left(T^{b} z\right)^{j} \mathcal{W}_{a b}+i \rho_{\bar{m} i j} \mathcal{D}_{\mu} z^{j} \mathcal{D}_{\nu} \bar{z}^{\bar{m}}\left(T^{a} z\right)^{i}\left(F_{a}^{\mu \nu}-i \tilde{F}_{a}^{\mu \nu}\right)+\text { h.c. }\right] \\
& +\left\{\rho_{i j} \mathcal{D}^{\mu} z^{j}\left[\frac{2}{x} \bar{f}_{\bar{m}} \mathcal{D}_{\mu} \bar{z}^{\bar{m}}\left(T^{a} z\right)^{i} \mathcal{D}_{a}-\frac{\bar{f}^{i}}{2} \mathcal{W} f_{j} \mathcal{D}_{\mu} z^{j}\right]+\text { h.c. }\right\} \\
& -\frac{i}{2} K_{i \bar{m}}\left[\mathcal{D}^{\nu} \bar{z}^{\bar{m}}\left(T_{a} z\right)^{i}-\mathcal{D}^{\nu} z^{i}\left(T_{a} z\right)^{\bar{m}}\right]\left[\bar{f}^{i} \rho_{i j} \mathcal{D}^{\rho} z^{j}\left(F_{\rho \nu}^{a}-i \tilde{F}_{\rho \nu}^{a}\right)+\text { h.c. }\right] \\
& +\frac{\mathcal{D}_{a}}{2 x}\left[K_{k \bar{m}} \mathcal{D}^{\mu} \bar{z}^{\bar{m}}\left(T^{a} z\right)^{k}+\frac{i}{2} \bar{f}_{\bar{m}} \mathcal{D}_{\nu} \bar{z}^{\bar{m}}\left(F^{\nu \mu}-i \tilde{F}^{\nu \mu}\right)+\text { h.c. }\right]\left(\rho_{i j} \mathcal{D}_{\mu} z^{i} \bar{f}^{j}+\text { h.c. }\right) \\
& -\left[\mathcal{W}^{a b} \rho_{i j} \bar{f}^{i}\left(T_{a} z\right)^{j} \mathcal{D}_{b}+x^{2} \mathcal{D}_{\rho} z^{i} \mathcal{D}^{\rho} z^{j}\left(2 \rho_{i j} \mathcal{W}-R_{\bar{n} i \bar{m} j} \rho^{\bar{m} \bar{n}} \overline{\mathcal{W}}\right)+\text { h.c. }\right] \\
& +2 x^{2} \rho_{i j} \rho_{\bar{m}}^{j} \mathcal{D} \mathcal{D}_{\rho} z^{i} \mathcal{D}^{\rho} \bar{z}^{\bar{m}} \\
& L_{g}=\frac{e^{-K}}{4 x^{2}}\left(\mathcal{D}_{\mu} z^{i} \mathcal{D}^{\mu} \bar{z}^{\bar{m}}+F^{i} \bar{F}^{\bar{m}}\right) A_{i j} \bar{A}_{\bar{m}}^{k} f_{k} \bar{f}^{j}+\frac{2}{9} M \bar{M}\left(\rho_{i j} \mathcal{D}_{\mu} z^{i} \mathcal{D}^{\mu} z^{j}+\text { h.c. }\right) \\
& -\frac{e^{-K / 2}}{8 x^{3}}\left[\bar{f}^{j} A_{i j} F^{k} f_{k} \bar{f}_{\bar{m}}\left(\mathcal{D}_{\mu} z^{i} \mathcal{D}^{\mu} \bar{z}^{\bar{m}}-F^{i} \bar{F}^{\bar{m}}\right)+\text { h.c. }\right] \\
& -\frac{e^{-K / 2}}{8 x^{3}}\left\{\left\{\bar{f}^{j} A_{i j} F^{k} f_{k}\left[\mathcal{D}_{\mu} z^{i} f_{\ell} \mathcal{D}^{\mu} z^{\ell}-\frac{4 x}{3} F^{i} M\right]+\text { h.c. }\right\}\right. \\
& -\frac{e^{-K / 2}}{2}\left\{\bar{f}^{\ell} A_{k \ell}\left[\frac{\bar{f}_{\bar{m}}}{3 x^{2}} \mathcal{D}^{\mu} \bar{z}^{\bar{m}} \mathcal{D}_{\mu} z^{\ell} \bar{M}-\rho_{i j} \mathcal{D}_{\mu} z^{i}\left(F^{j} \mathcal{D}^{\mu} z^{k}-F^{k} \mathcal{D}^{\mu} z^{j}\right)\right]+\text { h.c. }\right\} \\
& +\frac{1}{2 x^{2}}\left\{\mathcal{D}_{\mu} z^{i} \bar{F}_{i}\left[\frac{x}{3} \bar{M}\left(2 \bar{f}_{\bar{m}} \mathcal{D}^{\mu} \bar{z}^{\bar{m}}-f_{j} \mathcal{D}^{\mu} z^{j}\right)+f_{j} F^{j} \bar{f}_{\bar{m}} \mathcal{D}^{\mu} \bar{z}^{\bar{m}}\right]+\text { h.c. }\right\} \\
& +\frac{1}{4 x^{2}}\left[\mathcal{D}_{\mu} z^{i} \mathcal{D}^{\mu} z^{j} f_{i} f_{j}\left(\frac{1}{9} M \bar{M}+\frac{1}{4 x^{2}} f_{k} F^{k} \bar{f}_{\bar{m}} \bar{F}^{\bar{m}}+\frac{1}{6 x} f_{k} F^{k} M\right)+\text { h.c. }\right] \\
& -\left\{\frac{f_{i} f_{j} \bar{f}_{\bar{m}}}{24 x^{3}}\left[\left(\mathcal{D}_{\mu} z^{i} \mathcal{D}^{\mu} \bar{z}^{\bar{m}}+2 F^{i} \bar{F}^{\bar{m}}\right) F^{j} M-2 \mathcal{D}_{\mu} z^{i} \mathcal{D}^{\mu} z^{j} \bar{F}^{\bar{m}} \bar{M}\right]+\text { h.c. }\right\} \\
& -\frac{1}{4 x}\left\{\rho _ { i j } \overline { f } ^ { \ell } \overline { F } _ { \ell } \mathcal { D } _ { \mu } z ^ { i } \left[f_{k}\left(F^{j} \mathcal{D}^{\mu} z^{k}+F^{k} \mathcal{D}^{\mu} z^{j}\right)\right.\right. \\
& \left.\left.+\bar{f}_{\bar{n}} F^{j} \mathcal{D}^{\mu} \bar{z}^{\bar{n}}+\frac{4 x}{3} \mathcal{D}^{\mu} z^{j} \bar{M}\right]+ \text { h.c. }\right\} \\
& +\frac{f_{k} \bar{f}^{k}}{4 x}\left\{\mathcal{W}\left[x \rho_{i j} \mathcal{D}_{\mu} z^{i} \mathcal{D}^{\mu} z^{j}-\frac{e^{-K / 2}}{2 x} f_{j} \bar{A}_{\bar{m}}^{j} \bar{F}^{\bar{m}}-\frac{\bar{f}_{\bar{m}} f_{j}}{4 x^{2}} F^{j} \bar{F}^{\bar{m}}-\frac{f_{i}}{3 x} F^{i} M\right]+\text { h.c. }\right\} \\
& -\frac{1}{3} K_{i \bar{m}} K_{j \bar{n}}\left(\mathcal{D}_{\mu} z^{i} \mathcal{D}^{\mu} \bar{z}^{\bar{m}} \mathcal{D}_{\nu} z^{j} \mathcal{D}^{\nu} \bar{z}^{\bar{n}}+F^{i} \bar{F}^{\bar{m}} F^{j} \bar{F}^{\bar{n}}-F^{j} \bar{F}^{\bar{n}} \mathcal{D}_{\mu} z^{i} \mathcal{D}^{\mu} \bar{z}^{\bar{m}}\right)
\end{aligned}
$$




$$
\begin{aligned}
& +\frac{1}{6} K_{i \bar{m}} K_{j \bar{n}} \mathcal{D}_{\mu} z^{i} \mathcal{D}_{\nu} \bar{z}^{\bar{m}}\left(4 \mathcal{D}^{\mu} z^{j} \mathcal{D}^{\nu} \bar{z}^{\bar{n}}+\mathcal{D}^{\nu} z^{j} \mathcal{D}^{\mu} \bar{z}^{\bar{n}}\right)-\frac{1}{27}(M \bar{M})^{2}+\frac{2}{9} \mathcal{D} M \bar{M} \\
& +\left(\frac{1}{9} M \bar{M}-\frac{1}{3} \mathcal{D}\right) K_{i \bar{m}}\left(2 F^{i} \bar{F}^{\bar{m}}-\mathcal{D}_{\mu} z^{i} \mathcal{D}^{\mu} \bar{z}^{\bar{m}}\right)+\frac{2}{3} \mathcal{D}^{2}+x^{2} \mathcal{W}_{a b} \overline{\mathcal{W}}^{a b} \\
& +\left(F_{\rho \mu}^{a}+i \tilde{F}_{\rho \mu}^{a}\right)\left(F_{a}^{\rho \nu}-i \tilde{F}_{a}^{\rho \nu}\right)\left(\frac{f_{i} \bar{f}_{\bar{m}}}{4 x}-\frac{x}{2} K_{i \bar{m}}\right) \mathcal{D}_{\nu} z^{i} \mathcal{D}^{\mu} \bar{z}^{\bar{m}}+\left(\frac{f_{i} \bar{f}^{i}}{4 x}\right)^{2} \mathcal{W} \overline{\mathcal{W}} \\
& +x^{2} \rho_{i j} \rho_{\bar{n} \bar{m}}\left(\mathcal{D}_{\mu} z^{i} \mathcal{D}^{\mu} z^{j} \mathcal{D}_{\nu} \bar{z}^{\bar{m}} \mathcal{D}^{\nu} \bar{z}^{n}+\mathcal{D}_{\mu} z^{i} \mathcal{D}^{\mu} \bar{z}^{\bar{m}} F^{j} \bar{F}^{\bar{n}}\right) \\
& +\frac{f_{i} f_{j} \bar{f}_{\bar{m}} \bar{f}_{\bar{n}}}{16 x^{4}}\left(\mathcal{D}_{\mu} z^{i} \mathcal{D}^{\mu} z^{j} \mathcal{D}_{\nu} \bar{z}^{\bar{m}} \mathcal{D}^{\nu} \bar{z}^{\bar{n}}-F^{i} F^{j} \bar{F}^{\bar{m}} \bar{F}^{\bar{n}}-2 \mathcal{D}_{\mu} z^{i} \mathcal{D}^{\mu} \bar{z}^{\bar{m}} F^{j} \bar{F}^{\bar{n}}\right) \\
& +\frac{f_{j} \bar{f}_{\bar{n}}}{12 x^{2}} F^{j} \bar{F}^{\bar{n}}\left[K_{i \bar{m}}\left(\mathcal{D}_{\mu} z^{i} \mathcal{D}^{\mu} \bar{z}^{\bar{m}}-2 F^{i} \bar{F}^{\bar{m}}\right)+2 M \bar{M}-2 \mathcal{D}\right] \\
& +\frac{f_{j} \bar{f}_{\bar{n}}}{6 x^{2}} \mathcal{D}_{\nu} z^{j} \mathcal{D}^{\nu} \bar{z}^{\bar{n}}\left[K_{i \bar{m}}\left(2 \mathcal{D}_{\mu} z^{i} \mathcal{D}^{\mu} \bar{z}^{\bar{m}}-F^{i} \bar{F}^{\bar{m}}\right)+\frac{1}{2} M \bar{M}-\mathcal{D}\right] \\
& -\frac{f_{j} \bar{f}_{\bar{m}}}{2 x^{2}} K_{j \bar{n}}\left(\mathcal{D}_{\mu} z^{i} \mathcal{D}^{\mu} z^{j} \mathcal{D}_{\nu} \bar{z}^{\bar{m}} \mathcal{D}^{\nu} \bar{z}^{\bar{n}}+\mathcal{D}_{\mu} z^{i} \mathcal{D}^{\mu} \bar{z}^{\bar{n}} \mathcal{D}_{\nu} \bar{z}^{\bar{m}} \mathcal{D}^{\nu} z^{j}\right) \\
& +\frac{1}{2}\left(\mathcal{W}_{a b}+\overline{\mathcal{W}}_{a b}\right) \mathcal{D}^{a} \mathcal{D}^{b}
\end{aligned}
$$

where $F^{i}=-e^{-K / 2} \bar{A}^{i}$ is the bosonic part of the $F$-component of the chiral supermultiplet $Z^{i}, M=-3 e^{-K / 2} A$ is an auxiliary field in the gravity supermultiplet (see eq. $(2.53-56))$. The results of $[30,31]$ were calculated using the classical Lagrangian (1) that is obtained after elimination of the auxiliary fields, and are expressed in those papers as functions of the boson fields and their covariant derivatives. It is easy to show that calculating the one loop corrections before or after elimination of the auxiliary fields in terms of their classical solutions gives the same result to the loop order considered. Here we use the auxiliary fields to present the results in a form that lends itself more easily to an interpretation in terms of superfield operators.

The real function $K_{R}(Z, \bar{Z})$, given in eq. (4.80), contains logarithmically divergent contributions to the the renormalized Kähler potential. In $L_{2, g}$ we have also introduced scalar field reparameterization invariant covariant derivatives $\left(\rho_{i j}, \rho_{\bar{m} i j}\right)$ of the variable $\rho$, defined as the squared gauge coupling $\rho=x^{-1}=g^{2}$.

In effective supergravity from superstring theory, the classical Kähler potential $K(z, \bar{z})$, superpotential $W(z)$ and Yang-Mills normalization function $f_{a b}(z)$ take the forms given in eq. (4.86). In this case $1-(4 x)^{-2} \bar{f}^{i} f_{i}=A+(2 x)^{-1} \bar{f}^{i} A_{i}=0$, and $\rho_{i}=D_{i} \rho=-\left(2 x^{2}\right)^{-1} f_{i}$ is covariantly constant: $\rho_{i j}=\rho_{\bar{m} i j}=\cdots=0$. Then $L_{2}$ and $L_{g}$ reduce to:

$$
L_{2}=\left(\mathcal{W}^{a b}+\overline{\mathcal{W}}^{a b}\right)\left[3 C_{G} \delta_{a b}-D_{i}\left(T_{b} z\right)^{j} D_{j}\left(T_{a} z\right)^{i}\right]-24 i \mathcal{D}_{\mu} z^{i} \mathcal{D}_{\nu} \bar{z}^{\bar{m}} K_{i \bar{m}} \mathcal{D}^{a} F_{a}^{\mu \nu}
$$




$$
\begin{aligned}
& +\frac{N+5}{12}\left[(s+\bar{s})^{2} \mathcal{W}_{a b} \overline{\mathcal{W}}^{a b}+2\left(\mathcal{W}^{a b}+\overline{\mathcal{W}}^{a b}\right) \mathcal{D}_{a} \mathcal{D}_{b}+8 \mathcal{D}^{2}\right]+\frac{7}{2}(s+\bar{s})^{2} \mathcal{W}_{a b} \overline{\mathcal{W}}^{a b} \\
& -\frac{N+5}{3} \mathcal{D}\left[K_{i \bar{m}}\left(2 F^{i} \bar{F}^{\bar{m}}+\mathcal{D}_{\mu} z^{i} \mathcal{D}^{\mu} \bar{z}^{\bar{m}}\right)-\frac{2}{9} M \bar{M}\right]+11\left(\mathcal{W}^{a b}+\overline{\mathcal{W}}^{a b}\right) \mathcal{D}_{a} \mathcal{D}_{b} \\
& +\frac{(s+\bar{s})}{2}(\mathcal{W}+\overline{\mathcal{W}})\left[\frac{2}{3} M \bar{M}+K_{i \bar{m}}\left(\mathcal{D}_{\rho} z^{i} \mathcal{D}^{\rho} \bar{z}^{\bar{m}}-2 F^{i} \bar{F}^{\bar{m}}\right)+2 \mathcal{D}\right]+20 \mathcal{D}^{2} \\
& -\frac{N+2}{12}(s+\bar{s})\left(F_{\rho \mu}^{a}-i \tilde{F}_{\rho \mu}^{a}\right)\left(F_{a}^{\rho \nu}+i \tilde{F}_{a}^{\rho \nu}\right) \mathcal{D}_{\nu} z^{i} \mathcal{D}^{\mu} \bar{z}^{\bar{m}} K_{i \bar{m}}+\frac{154}{9} M \bar{M} \mathcal{D} \\
& +2 K_{i \bar{m}}\left(13 F^{i} \bar{F}^{\bar{m}}+9 \mathcal{D}_{\mu} z^{i} \mathcal{D}^{\mu} \bar{z}^{\bar{m}}\right) \mathcal{D}-\frac{2 e^{-K / 2}}{(s+\bar{s})} \mathcal{D}_{a}\left[\left(T^{a} z\right)^{i} R_{i}^{j}{ }^{k}{ }^{k} F^{\ell} A_{j k}+\text { h.c. }\right] \\
& -\left[\frac{4}{3} \mathcal{D} R_{i \bar{m}}+\frac{\mathcal{D}_{a}}{(s+\bar{s})}\left(R_{\bar{n} i \bar{m} j} D^{j}\left(T^{a} \bar{z}\right)^{\bar{n}}-\frac{4}{3} K_{i \bar{m}} D_{j}\left(T^{a} z\right)^{j}\right)\right] \\
& \times\left(F^{i} \bar{F}^{\bar{m}}+\mathcal{D}_{\mu} z^{i} \mathcal{D}^{\mu} \bar{z}^{\bar{m}}\right)+28 i \frac{\partial_{\mu} s \partial_{\nu} \bar{s}}{(s+\bar{s})^{2}} \mathcal{D}^{a} F_{a}^{\mu \nu} \\
& +\frac{D_{j}\left(T^{a} z\right)^{j}}{3}\left[13 i K_{\bar{m} i} F_{\mu \nu}^{a} \mathcal{D}^{\mu} z^{i} \mathcal{D}^{\nu} \bar{z}^{\bar{m}}-\frac{8 \mathcal{D}_{a} \mathcal{D}}{(s+\bar{s})}\right]+2 i F_{\mu \nu}^{a} D_{j}\left(T_{a} z\right)^{i} R_{i \bar{m} k}^{j} \mathcal{D}^{\mu} z^{k} \mathcal{D}^{\nu} \bar{z}^{\bar{m}} \\
& -\frac{12}{s+\bar{s}}\left\{\left[i \partial_{\nu} s\left(F_{a}^{\nu \mu}-i \tilde{F}_{a}^{\nu \mu}\right)+\frac{2 \partial^{\mu} s}{s+\bar{s}} \mathcal{D}_{a}\right] \mathcal{D}_{\mu} \bar{z}^{\bar{m}} K_{i \bar{m}}\left(T^{a} z\right)^{i}+\text { h.c. }\right\} \\
& -2 \frac{\partial_{\rho} s \partial^{\nu} \bar{s}}{(s+\bar{s})}\left(F_{\mu \nu}^{a}+i \tilde{F}_{\mu \nu}^{a}\right)\left(F_{a}^{\mu \rho}-i \tilde{F}_{a}^{\mu \rho}\right)+40 \frac{\partial_{\mu} s \partial^{\mu} \bar{s}}{(s+\bar{s})^{2}} \mathcal{D} \\
& L_{g}=\frac{(s+\bar{s})^{2}}{4}\left(\mathcal{W} \overline{\mathcal{W}}+\mathcal{W}_{a b} \overline{\mathcal{W}}^{a b}\right)+\frac{1}{2}\left(\mathcal{W}_{a b}+\overline{\mathcal{W}}_{a b}\right) \mathcal{D}^{a} \mathcal{D}^{b}+\frac{2}{3} \mathcal{D}^{2} \\
& +\frac{1}{6} K_{i \bar{m}} K_{j \bar{n}}\left(4 \mathcal{D}_{\mu} z^{i} \mathcal{D}^{\mu} z^{j} \mathcal{D}_{\nu} \bar{z}^{\bar{m}} \mathcal{D}^{\nu} \bar{z}^{\bar{n}}+\mathcal{D}_{\mu} z^{i} \mathcal{D}^{\mu} \bar{z}^{\bar{n}} \mathcal{D}_{\nu} \bar{z}^{\bar{m}} \mathcal{D}^{\nu} z^{j}\right) \\
& -\frac{1}{3} K_{i \bar{m}} K_{j \bar{n}}\left(\mathcal{D}_{\mu} z^{i} \mathcal{D}^{\mu} \bar{z}^{\bar{m}} \mathcal{D}_{\nu} z^{j} \mathcal{D}^{\nu} \bar{z}^{\bar{n}}+F^{i} \bar{F}^{\bar{m}} F^{j} \bar{F}^{\bar{n}}-F^{j} \bar{F}^{\bar{n}} \mathcal{D}_{\mu} z^{i} \mathcal{D}^{\mu} \bar{z}^{\bar{m}}\right) \\
& +\left(\frac{2}{27} M \bar{M}-\frac{1}{3} \mathcal{D}\right) K_{i \bar{m}}\left(2 F^{i} \bar{F}^{\bar{m}}-\mathcal{D}_{\mu} z^{i} \mathcal{D}^{\mu} \bar{z}^{\bar{m}}\right)-\frac{2}{27} \mathcal{D} M \bar{M} \\
& -\frac{1}{81}(M \bar{M})^{2}-(s+\bar{s})(\mathcal{W}+\overline{\mathcal{W}})\left(\frac{1}{2} F^{i} \bar{F}^{\bar{m}} K_{i \bar{m}}-\frac{1}{9} M \bar{M}\right) \\
& +\frac{2 \partial_{\nu} s \partial^{\nu} \bar{s}}{3(\bar{s}+\bar{s})^{2}}\left[K_{i \bar{m}}\left(2 \mathcal{D}_{\mu} z^{i} \mathcal{D}^{\mu} \bar{z}^{\bar{m}}-F^{i} \bar{F}^{\bar{m}}\right)+\frac{1}{3} M \bar{M}-\mathcal{D}\right] \\
& +\frac{1}{(s+\bar{s})^{4}} \partial_{\mu} s \partial^{\mu} s \partial_{\nu} \bar{s} \partial^{\nu} \bar{s}-\frac{2 \partial_{\mu} s \partial_{\nu} \bar{s}}{(s+\bar{s})^{2}} K_{j \bar{n}}\left(\mathcal{D}^{\mu} z^{j} \mathcal{D}^{\nu} \bar{z}^{\bar{n}}+\mathcal{D}^{\mu} \bar{z}^{\bar{n}} \mathcal{D}^{\nu} z^{j}\right) \\
& +\left(F_{\rho \mu}^{a}+i \tilde{F}_{\rho \mu}^{a}\right)\left(F_{a}^{\rho \nu}-i \tilde{F}_{a}^{\rho \nu}\right)\left(\frac{\partial_{\nu} s \partial^{\mu} \bar{s}}{2(s+\bar{s})}-\frac{s+\bar{s}}{4} K_{i \bar{m}} \mathcal{D}_{\nu} z^{i} \mathcal{D}^{\mu} \bar{z}^{\bar{m}}\right)
\end{aligned}
$$

with now:

$$
\begin{aligned}
K_{R} & =K+\frac{\ln \Lambda^{2}}{32 \pi^{2}}\left\{e^{-K}\left[A_{i j} \bar{A}^{i j}-2 A_{i} \bar{A}^{i}+\left(N_{G}-4\right) A \bar{A}\right]-4 \mathcal{K}-16 \mathcal{D}\right\}, \\
M & =-\frac{3}{s+\bar{s}} \bar{F}^{\bar{s}}, \quad M \bar{M}=9 F^{s} \bar{F}_{s},
\end{aligned}
$$

where the second line follows from the tree level equations of motion.

In addition, in the untwisted sector of orbifold compactifications, the Riemann ten- 
sor is covariantly constant and its elements are related to elements of the Kähler metric. Moreover in many models there are global symmetries that impose $R^{i \bar{m} j \bar{n}} W_{i j}=$ $R^{i \bar{m} j \bar{n}} W_{i j k}=0$. In this case $L_{1}$ can be expressed entirely in terms of $F^{i}, \mathcal{D}_{\mu} z^{i}, M$, their complex conjugates, and the Kähler metric; an explicit example is given in [30].

\subsection{Conclusions}

In this chapter we have shown the calculation and the result of the divergent loop correction [31] to supergravity and superstring effective models, including the gauge sector.

Some comments on the implications and applications of our results are in order. It has already been shown [45] that, using the gauge fixing and expansion procedures defined here, the one-loop quadratic divergences, as well as the logarithmic divergences in the flat space limit and in the absence of a dilaton, can be regulated à la Pauli-Villars. Regularization of the full supergravity divergences without a dilaton are under study [52]. An objective of this study is to determine the extent to which, in the string theory context, a modular invariant regularization procedure can be achieved that preserves the continuous $S L(2, R)$ symmetry of the classical effective Lagrangian. To obtain the full one-loop Lagrangian, including all finite contributions, requires a resummation of the derivative expansion. A procedure for resummation will be described elsewhere [52].

We have presented our results for one-loop corrections to the classical general supergravity Lagrangian $[42,13]$ with at most two-derivative terms. As seen in Section 5 , the result simplifies considerably for the classical effective Lagrangian derived from string theory, due to the the absence of a potential for the dilaton and the special form of its Kähler potential. These features are modified when the effective Lagrangian includes a nonperturbatively induced [72] superpotential for the dilaton and/or the Green-Schwarz counterterm [26] that is necessary to restore modular invariance. The latter term destroys the no-scale nature of Lagrangians from torus compactification and the untwisted sector of orbifold compactification, and generally destabilizes the effective scalar potential. However this term is of one-loop order and therefore should be considered together with the full one-loop corrections. An interesting question, that will be addressed elsewhere, is whether these corrections can restabilize the potential.

An important unresolved issue in the construction of effective supergravity Lagrangians for gaugino condensation is the correct form of the kinetic term for the com- 
posite chiral multiplet that represents the lightest bound state of the confined Yang-Mills sector. It has recently been shown [27], in the context of both the linear and chiral multiplet formulations for the dilaton, that such terms can be generated by higher dimension operators. The contribution (4.84) to the effective Lagrangian determines the leading one-loop contribution to these operators; similar terms occur in string theory [65]. This is one example of how the determination of loop corrections can serve as guide to the construction of such an effective theory. 


\section{Chapter 5}

\section{Gaugino Condensation, Threshold Corrections, and S-Duality}

\subsection{Introduction}

As discussed in Section 2.1, a basic feature of superstring constructions in four dimensions is the presence of massless moduli in the effective field theory. These fields whose vevs parameterize the continuously degenerate string vacua, are gauge-singlet chiral fields; furthermore, they are exact flat directions of the low energy effective field theory (LEEFT) scalar potential. Generically, the moduli appear in the couplings of the LEEFT. For example, the tree level gauge couplings at the string scale depend on the dilaton, $S$, and the Yukawa couplings as well as the kinetic terms depend on the $T$-moduli (and $S$ through the Kähler potential) . There is mixing of the moduli beyond tree level, due to both string threshold corrections [25] and field-theoretical loop effects.

Since the supersymmetric vacua of heterotic strings consist of continuously degenerate families (to all orders of perturbation theory), parameterized by the moduli vevs, the latter remain perturbatively undetermined. This degeneracy can only be lifted by a nonperturbative mechanism which would induce a nontrivial superpotential for moduli, and at the same time break supersymmetry. We shall assume that this nonperturbative mechanism takes place in the LEEFT and is not intrinsically stringy. This certainly appears to be the most "tractable" possibility. A popular candidate for such a mechanism has been gaugino condensation which is briefly reviewed in Section 5.2.

In this Chapter, we wish to consider gaugino condensation in a superstring-inspired effective field theory, with approximate $S$-duality invariance $[66,67]$ and exact T-modular invariance. We generalize the work in ref. [67] to incorporate an intermediate scale $M_{I}$ ( $M_{\text {cond }} \ll M_{I} \ll M_{\text {string }}$ ), and we are interested in how the intermediate-scale threshold corrections will affect gaugino condensation and supersymmetry breaking[85]. The intermediate scale may be generated by spontaneous breaking of the underlying gauge symmetry, or alternatively, by a gauge singlet field, $A$, which is coupled to the hiddensector gauge non-singlet fields $\Phi_{i}$. In the latter scheme, $A$ is assumed to acquire a VEV 
dynamically and therefore gives the gauge non-singlet fields masses without breaking the gauge group. We assume the latter scheme because of its simplicity. In fact, this scheme has been seriously considered when studying the gauge coupling unification [69]. Incorporating the intermediate-scale threshold corrections into gaugino condensation is non-trivial in the sense that the field-theoretical threshold corrections at $M_{I}$ are dilaton-dependent. Hence, these modifications can have non-trivial implications for supersymmetry breaking by gaugino condensation. Furthermore, a priori, nothing prohibits intermediate scales in the hidden sector.

The outline of this chapter is as follows. After a brief review of gaugino condensation (Section 5.2), and of duality symmetries, modular and S-duality in Section 5.3, we shall review the quantum corrections to the gauge couplings in superstring effective supergravity theories and the connection with modular invariance of the effective theory in Section 5.4. We shall discuss our model in Section 5.5, and arrive at the renormalized Kähler potential including 1-loop threshold corrections at an intermediate mass, and constrained by duality symmetries. The issues related to the scalar potential, dilaton run-away, and supersymmetry breaking, as well as the role of the intermediate mass are discussed in Section 5.5. Concluding remarks are given in Section 5.6.

\subsection{Gaugino Condensation (A Review)}

A possible mechanism for breaking supersymmetry within the framework of $(N=1$, $D=4$ ) LEEFT of superstring is gaugino condensation in the hidden sector. In this scenario, the nonperturbative effects arise from the strong coupling of the asymptotically free gauge interactions at energies well below $M_{P l}$. Corresponding to this strong coupling is the condensation of gaugino bilinear $\langle\bar{\lambda} \lambda\rangle_{h . s .}$. Let us briefly remind the reader the overview of the development of gaugino condensation. It was recognized many years ago that gaugino condensation in globally supersymmetric Yang-Mills theories without matter does not break supersymmetry [70]. In fact, that dynamical supersymmetry breaking cannot be achieved in pure SYM theories was shown by topological arguments of Witten [71]. In the locally supersymmetric case the picture is rather different, namely, gaugino condensation can break supersymmetry [72], and the gauge coupling is itself generally field-dependent. When the gauge coupling becomes strong, it gives rise to 
gaugino condensation at the scale ${ }^{1}$

$$
M_{\text {cond }} \sim M_{\text {string }}\langle\operatorname{Re} T\rangle^{-1 / 2} e^{-\operatorname{Re} S / 2 b_{0}}=M_{\text {string }}\langle\operatorname{Re} T\rangle^{-1 / 2} e^{-1 / b_{0} g_{\text {st }}^{2}}
$$

which breaks local supersymmetry spontaneously $\left(M_{\text {cond }}^{3} \sim\langle\bar{\lambda} \lambda\rangle_{h . s .}\right)$, and $S$ is the dilaton/axion chiral field. Supersymmetry breaking in the observable sector is induced by gravitational interactions which act as 'messenger' between the two otherwise decoupled sectors.

However, there are generally two problems associated with the above scenario. First, the destabilization of $S$ - the only stable minimum of the potential in the $S$-direction being at $S \rightarrow \infty$; i.e., in the direction where exact supersymmetry is recovered and the coupling vanishes! This is contrary to the expectation that the vacuum is in the strongly coupled, confining regime. This problem, the so-called dilaton runaway problem, is present in most formulations of gaugino condensation, in particular the so-called 'truncated superpotential' approach [74], where the condensate field is assumed to be much heavier than the dilaton and therefore is integrated out below $M_{\text {cond }}$. In fact, the dilaton runaway problem is perhaps a more generic problem in string phenomenology where the underlying string theory is assumed to be weakly coupled without any nonperturbative effects being taken into account [86]. We shall return to the dilaton runaway in Sections 5 and 6.

The second difficulty is the large cosmological constant that arises from the vacuum energy associated with gaugino condensation. An early attempt to remedy these difficulties was proposed by Dine et al. [74], in the context of no-scale supergravity whereby a constant term, $c$, is introduced in the superpotential which independently breaks supersymmetry and cancels the cosmological constant. The origin of $c$ is traced to the vev of the 3-form in 10D supergravity, and is quantized in units of order $M_{p l}$. Therefore, this approach has the unsatisfactory feature of breaking supersymmetry at the scale of the fundamental theory.

\subsection{Duality Symmetries}

Modular symmetry, with the group $S L(2, \mathcal{Z})$ subgroup of $S L(\dot{2}, \mathcal{R})$ duality transfor-

\footnotetext{
${ }^{1}$ These arguments are modified by, for instance, the requirement of modular invariance [73].
} 
mations, written in its simplest form:

$$
T \rightarrow \frac{\alpha T-i \beta}{i \gamma T+\delta}
$$

where $\alpha \delta-\beta \gamma=1$ and $\alpha, \beta, \gamma, \delta$ are integers, ${ }^{2}$ is an exact invariance of the underlying string theory. However, this symmetry is anomalous in the LEEFT. Cancellation, or partial cancellation, of this anomaly in the effective theory can be achieved by the GreenSchwarz (GS) mechanism, which is especially clear in the linear-multiplet formulation of the LEEFT $[75,76,77]$. In the corresponding chiral formulation, the adding of GS counter-terms amounts to modifying the dilaton Kähler potential:

$$
-\ln (S+\bar{S}) \rightarrow-\ln (S+\bar{S}-b G)
$$

where $b=-\frac{2}{3} b_{0}$, and $b_{0}$ is the $E_{8}$ one-loop $\beta$-function coefficient. $G=\Sigma_{i} \ln \left(T^{i}+\right.$ $\bar{T}^{i}-\Sigma|\Phi|^{2}$ ), and $\Phi$ is any untwisted sector (non-modulus) chiral field of modular weight $(1,1,0)$ in the theory. We neglect the twisted dector matter fields. For simplicity, here we only consider models where modular anomalies are completely cancelled by GS mechanism, for example, the $(2,2)$ symmetric abelian orbifolds with no $N=2$ fixed planes, like $Z_{3}$ or $Z_{7}[75,76,77]$. The role of the gauge coupling and its renomalization in superstring effective theories, and the connection with modular anomaly cancellation are reviewed in the next section.

Recently, another type of duality symmetry has been receiving much attention in string theories. In this case the group of duality transformations is $S L(2, \mathcal{Z})$, but acting on the field $S$ instead of $T^{i}$, and is referred to as $S$-duality. Like its $T$-analogue, this group has a generator which is the transformation $S \rightarrow 1 / S$, and since $S$ is related to the gauge coupling, this duality transformation is also referred to as 'strong-weak' duality. Font et al. [78] have conjectured that $S$-duality, like $T$-duality is an exact symmetry of string theory. More recently, there has been mounting evidence that $S$ duality is a symmetry of certain string theories [79]. However, these theories all have $N=4$ or $N=2$ supersymmetries. At the level of string theory, there are two different types of S-duality, namely $(i)$ those that map different theories into one another, and (ii) those that map strongly and weakly coupled regimes of the same theory into each another. Indeed, presently there is no evidence of an S-dual $N=1$ string theory, and it is therefore difficult to justify the use of S-duality as a true symmetry in the

\footnotetext{
${ }^{2}$ There is, generally, one copy of the group per modulus field $T^{i}$.
} 
corresponding LEEFT. However, it has been shown that in the effective theory, the full $S L(2, \mathcal{R})$ duality transformation is a symmetry of the equations of motion of the gravity, gauge, and dilaton sector in the limit of weak gauge coupling [66, 67]. As in [67], we shall take S-duality as a guiding principle in constructing the Kähler potential for the gaugino condensate, which is, so far, the least understood element in the description of the effective theory for gaugino condensation. That is, we assume that S-duality invariance is recovered in limit of vanishing gauge coupling, $S+\bar{S} \rightarrow \infty$.

In the following we review some elements of S-duality transformations derived from the general formalism of ref. [66] (see also [67]). In the simplest case, in the presence of a YM field-strength $F_{\mu \nu}$, the scalar fields parameterize the coset space $G / H$, where $G=$ $S L(2, \mathcal{R})$, is the (noncompact) group of duality transformations and $H$ is its maximal compact subgroup $U(1)$. Under the action of $S L(2, \mathcal{R})$, the bosonic component of the dilaton transforms in the usual way:

$$
s \rightarrow s^{\prime}=\frac{a s-i b}{i c s+d}
$$

where $a, b, c, d$ are real, and $a d-b c=1$. The transformation of the fermions is determined by the considering the invariance of the corresponding kinetic terms and their coupling to the dilaton. One then obtains the transformation property of the supermultiplet. As shown in ref. [67], the transformation law (B.1) can be promoted to that of the dilaton (chiral) supermultiplet as follows:

$$
S(\theta) \rightarrow \frac{a S\left(\theta^{\prime}\right)-i b}{i c S\left(\theta^{\prime}\right)+d}=S^{\prime}\left(\theta^{\prime}\right)
$$

where

$$
\theta \rightarrow \theta^{\prime}=\left(\frac{i c s+d}{-i c \bar{s}+d}\right)^{1 / 2} \equiv \xi^{-1 / 2} \theta
$$

and

$$
\psi_{S} \rightarrow \psi_{S}^{\prime}=\xi^{-1 / 2}(i c s+d)^{-2} \psi_{S}
$$

Similarly, for the gaugino one finds:

$$
\lambda_{L} \rightarrow \xi^{1 / 2}(i c s+d) \lambda_{L},
$$

which implies that:

$$
W_{\alpha}(\theta) \rightarrow \xi^{1 / 2}(i c s+d) W_{\alpha}\left(\theta^{\prime}\right) ; \quad U(\theta) \rightarrow \xi(i c s+d)^{2} U\left(\theta^{\prime}\right)
$$


where $U$ is the composite field containing the gaugino condensate: $U=e^{K / 2} H^{3}$. Here, $H$ is the usual chiral multiplet. Note that $U$ and $H$ have different Kähler weights, therefore, $U$ differs from an ordinary chiral superfield; in fact it can be shown to satisfy the constraint $U=\left(\overline{\mathcal{D}}^{2}-8 R\right) V$, where $V$ is a vector multiplet which contains the components of a linear multiplet and a chiral multiplet ([67,64]).

It follows from the above transformation laws that the chiral field $H$ transforms as:

$$
H \rightarrow(i c s+d)^{1 / 3} H
$$

This, together with the fact that $\operatorname{Re} S \rightarrow|i c S+d|^{-2} \operatorname{Re} S$, fixes (up to an S-invariant factor) the function $f(S, \bar{S})$ in the Kähler potential (21): $f=(S+\bar{S})^{1 / 3}$. Notice that the $T$-moduli are inert under S-duality transformations.

There have been other recent discussions of gaugino condensation with S-duality [80] but with a rather different approach than ours; namely, by modifying the gauge kinetic term by replacing the gauge kinetic function $S$ with the function $S+1 / S$, and introducing a very different nonperturbative superpotential for the dilaton than one gets using the standard approach of ref. [70] as we do here. Other crucial differences with this work are the renormalization of the dilaton in Kähler function (including threshold corrections), and the use of $S L(2, R)$ approximate symmetry to constrain $K$ in our approach.

\subsection{The Role of the Gauge Coupling}

In this section, we recall a few facts about the perturbative corrections of the gauge coupling function in the superstring effective field theory as well as the connection with modular invariance of the effective theory.

As mentioned earlier, in our approach, the one-loop renormalization of the gauge coupling is completely included in the Kähler potential $K$, i.e., the renormalization effects are completely absorbed into $K$ by replacing the tree-level gauge coupling $S+\bar{S}$ in $K$ by the one-loop renormalized gauge coupling. Therefore, it is worthwhile to discuss the renormalization of gauge couplings in superstring LEEFTs. For simplicity and explicitness, we restrict ourselves to untwisted sector of orbifold models.

Let us first recall the Lagrangian for supergravity plus super-YM. Recall from eq. (2.26) and (2.28-29) that in the Kähler covariant formalism [12] the classical superfield 
Lagrangian is given by:

$$
\mathcal{L}=-3 \int d^{4} \theta E+\int d^{4} \theta\left(\frac{E}{8 R} f_{a b}(Z) W^{\alpha a} W_{\alpha}^{b}+\frac{E}{2 R} e^{K / 2} W(Z)\right)+\text { h.c. },
$$

where $Z$ stands for the chiral fields in the theory. The first term in eq. (5.9) corresponds to the kinetic energy for the gravity sector as well as the chiral fields. The chiral fields enter through the dependence of the spinorial derivatives of $E$ on the Kähler potential, $K(Z, \bar{Z})$. The second term describes the super-YM coupling to the theory, with the (holomorphic) gauge coupling function $f_{a b}(Z)$ and the YM 'field-strength' superfield

$$
W_{\alpha}=W_{\alpha a} T^{a}=\left(\frac{1}{8} \overline{\mathcal{D}}^{2}-R\right) e^{-2 V} \mathcal{D}_{\alpha} e^{2 V},
$$

where $V$ is the vector multiplet containing the YM gauge potential. We shall take $f_{a b}=f \delta_{a b}=S \delta_{a b}$ corresponding to the bare coupling of the effective superstring theories where $S$ is the dilaton/axion chiral superfield. The component form of the second term contains:

$$
\int d^{4} x \sqrt{g}\left(-\frac{1}{4} \operatorname{Re} f \operatorname{Tr}\left(F^{2}\right)-\frac{1}{4} \operatorname{Im} f \operatorname{Tr}(F \tilde{F})\right),
$$

and thus $\operatorname{Re} f$ is the YM gauge coupling, while $\operatorname{Im} f$ gives the axionic coupling.

Finally in the last term of eq. (5.9), $W(Z)$ is the superpotential which is a holomorphic function of the chiral matter fields (independent of $S$ and other internal moduli, until supersymmetry is broken nonperturbatively).

In discussing the gauge couplings in effective theories, it is important to to distinguish between the Wilsonian couplings, and the physical, or effective couplings. In particular in the effective supersymmetric theories that we are considering, there are powerful statements that can be made about the two types of gauge coupling. The (holomorphic) Wilsonian gauge couplings in supersymmetric YM theories, which appear in the Wilson effective action, do not renormalize beyond one loop. These are functions that appear in the Wilson effective action, $S_{W}(\mu)$, the local functional of quantum operators. In $S_{W}(\mu)$, only momenta between the scale $\mu$ and the UV cut-off contribute to loops. The physically measurable 'effective' (or running) couplings appear in the c-number valued generating functional of $1 \mathrm{PI}$ graphs, $\Gamma$; this is in general a nonlocal functional of background fields that contain the IR momenta $p<\mu$ running through loops, as well. Right at the UV cut off, the Wilsonian couplings, i.e., the coefficients appearing in front of the operator terms in $S_{W}$ are the bare couplings of the theory. The relation between the two effective actions may formally be written as [58]

$$
e^{i \Gamma\left[\Phi_{c \ell}, \mu\right]}=\left\langle e^{i S_{W}[\Phi, \mu]}\right\rangle
$$


where the expectation value on the right hand side is taken in the the presence of background fields. In the supersymmetric YM theories, it is known that, unlike the Wilsonian gauge coupling, the effective coupling renormalizes perturbatively at all orders, and that, indeed, higher order corrections introduce nonholomorphicities [58]. The generalizations of these results to supergravity effective theories of superstrings have been carried out more recently $[25,77,83,84]$.

The gauge coupling in all $N=1$ effective heterotic string constructions is given at tree level by:

$$
g_{\alpha}^{-2}=k_{\alpha} \operatorname{Re} S=k_{\alpha} g_{\text {string }}^{-2}
$$

$\operatorname{Re} S$ is the 'universal' gauge coupling at string scale, and $k_{\alpha}$ is the level of the affine Lie algebra associated with the factor $G_{\alpha}$ of the product gauge group. Subsequently, we shall set $k_{\alpha}=1$, and throughout the analysis $G_{\alpha}$ refers to the IR strong group with gaugino condensation. The exact (i.e., all-loop) Wilsonian coupling is given by the holomorphic function: $f_{W}=S+f^{(1)}$, and the moduli dependent one-loop (i.e., all-loop) correction $f^{(1)}\left(T^{i}\right)$ has been determined [84] (see below). The effective gauge coupling, with LEEFT-loop corrections to all orders is given by $[83,58]$ :

$$
\begin{aligned}
g_{e f f}^{-2}\left(p^{2}\right)=\operatorname{Re} S & +b_{0} \ln \frac{\Lambda^{2}}{p^{2}}+c K+\frac{T(a d j)}{8 \pi^{2}} \ln g_{e f f}^{-2}\left(p^{2}\right) \\
& -\frac{1}{8 \pi^{2}} \sum_{r} T(r) \ln \operatorname{det} Z_{e f f}^{(r)}\left(p^{2}\right)
\end{aligned}
$$

where, $b_{0} \equiv\left(-3 T(a d j)+\sum_{r} n_{r} T(r)\right) / 16 \pi^{2}$ (the YM $\beta$-function coefficient), and $c \equiv$ $\left(-T(a d j)+\sum_{r} n_{r} T(r)\right) / 16 \pi^{2}$, and $Z$ is the kinetic normalization matrix. It is worth mentioning that in our discussion, the above result for the (physical) effective gauge coupling is obtained by starting with the definition of Wilsonian coupling of ref. [83, 84] with a constant UV-cutoff. It can also be obtained by a Pauli-Villars regularization involving a field-dependent UV cutoff [77]. In the latter, definition of the Wilsonian coupling differs from the above, and due to the dependence of the the cut off on Kähler potential, it is non-holomorphic. To one-loop order, one has to evaluate the r.h.s. of the above equation at tree level, at 2-loop the r.h.s. is evaluated to one loop, etc. The one-loop result has also been obtained in [77]. Threshold corrections due to integrating out the heavy string modes have been calculated in reference [25]. These corrections are only dependent on the moduli $T^{i}$, and not on the dilaton. All the perturbative dilaton dependences in the effective gauge coupling arise from field-theoretical loop effects. We 
shall see in the next section that threshold corrections in the effective field theory also introduce dilaton-dependent terms in the running coupling.

Let us now turn to the question of modular invariance. As inputs from string theory, for general fields $\Phi^{I}$ (ignoring for the moment the GS counter terms), we have the normalization matrix for the kinetic term, and the Kähler function. The former is given by:

$$
Z_{I \bar{J}}=\delta_{I \bar{J}} \prod_{i}\left(T^{i}+\bar{T}^{i}\right)^{-q_{I}^{i}}+\mathcal{O}\left(\Phi^{2}\right)
$$

where the rational numbers $q_{I}^{i}$ are the modular weights of the field $\Phi^{I}$. They depend on the twist sector of the orbifold which gives rise to the matter fields $\Phi^{I}$, and the modulus field $T^{i}$. The Kähler function at the tree level is given by $K=-\ln (S+\bar{S})-\sum_{i} \ln \left(T^{i}+\right.$ $\left.\bar{T}^{i}\right)+\mathcal{O}\left(\Phi^{2}\right)$. For the modular transformation given in eq. (1) of the text, $K$ transforms by the usual transformation law:

$$
K \rightarrow K+F+\bar{F}, \quad F=\sum_{i} \ln \left(i \gamma_{i} T^{i}+\delta_{i}\right)
$$

Under a modular transformation, the non-modulus chiral fields, transforms as:

$$
\Phi^{I} \rightarrow C_{J}^{I}\left(T^{i}\right) \Phi^{J}
$$

Hence, the kinetic matrix $Z_{I \bar{J}}$ transforms according to:

$$
Z \rightarrow\left(C^{\dagger}\right)^{-1} Z C^{-1}
$$

It follows from eq. (5.13-15) that the reparametrization induced on the matter fields by modular transformations is given by:

$$
C_{J}^{I}=C_{0 J}^{I} \prod_{i}\left(i \gamma_{i} T^{i}+\delta_{i}\right)^{q_{I}^{i}}
$$

where $C_{0 J}^{I}$ is moduli independent.

For a generic supergravity theory with super-YM, under the combined transformations: $K \rightarrow K+F+\bar{F}$ and $\Phi^{I} \rightarrow C_{J}^{I} \Phi^{J}$ with $C_{J}^{I}$ holomorphic function of the moduli $\Phi^{I}$, the Kähler invariance of the (exact) integral of the RGE's, i.e., eq. (5.11) imply that:

$$
f_{W} \rightarrow f_{W}+c F-\frac{1}{2 \pi^{2}} \sum_{r} T(r) \operatorname{tr} \ln C^{(r)}
$$


where $c$ is the group theoretical factor given after eq. (5.11) above. For $C_{J}^{I}$ and $F$ corresponding to modular transformations, eq's (5.13) and (5.16), this gives:

$$
\operatorname{Re} f_{W} \rightarrow \operatorname{Re} f_{W}-\frac{1}{16 \pi^{2}} \sum_{i} 2 \alpha^{i} \ln \left|\left(i \gamma_{i} T^{i}+\delta_{i}\right)\right|^{2}
$$

with

$$
\alpha^{i}=\sum_{I} T\left(\Phi^{I}\right)\left(1-2 q_{I}^{i}\right)-T(a d j) ; \quad T\left(\Phi^{I}\right)=\sum_{r} \operatorname{tr}\left(T^{2}(r)\right)
$$

and $T_{a}(r)$ are the generators of the representations of the fields $\Phi^{I}$.

Furthermore, the transformation law (5.18) corresponds, up to a modular invariant function, to the transformation of the logarithm of Dedekind function. In fact it will give the complete modular dependent perturbative correction, $f^{(1)}$ to the Wilsonian coupling $[25,76,77]:$

$$
\operatorname{Re} f^{(1)}=-\frac{1}{4 \pi^{2}} \sum_{i} \alpha^{i} \ln \left|\eta\left(i T^{i}\right)\right|^{2},
$$

modulo a moduli independent part which has been argued to be a constant in most orbifold models [84].

In the context of the effective theory of gaugino condensation modular invariance is restored by including factors of $\eta\left(i T^{i}\right)$ in the superpotential (see eq (2)), and in the definition of the fields, so as to parametrize the above modular dependent correction of the gauge coupling, as well as by introducing GS counter term as discussed in section 2-B. However, the inclusion of the $\eta$ factors tends to spoil the boundedness from below of the scalar potential. To avoid this, we may restrict ourselves to the orbifold models which do not receive string threshold corrections. These models have been classified $[25,76,84]$. For such models, the modular anomaly is solely cancelled by the GS counter term.

\subsection{The Model}

This model basically generalizes the model of gaugino condensation with S-duality of ref. [67] to the case in the presence of an intermediate scale. Other works based on the truncated approach have addressed gaugino condensation in the presence of an intermediate scale [81]. However, our approach is quite different from those works in three respects. First, the effective Lagrangian approach is adopted here rather than the truncated approach. In the truncated approach, the mass of the composite is assumed to be much larger than the mass of the dilaton, and the condensate is integrated out below the condensation scale. Here, both the composite field and the dilaton are treated 
as dynamical fields. Due to this very assumption made in the truncated approach, these two approaches are not equivalent in the case where the mass of the composite is of the order the dilaton's mass or lower. Second, invariance under S-duality is used here to constrain those parts of the Lagrangian which cannot be obtained using the argument of anomalous symmetry. Third, the (dilaton dependent) one-loop intermediate-scale threshold corrections to the gauge coupling are included in this study.

The scheme of generating the intermediate scale considered here involves the coupling of the hidden-sector gauge non-singlet fields $\Phi_{i}$ to a gauge singlet $A$. When $A$ dynamically gets a vev, $\Phi_{i}$ become massive and the intermediate scale is thus generated. Since $A$ is a singlet, the hidden-sector gauge group does not break. Such a scheme has interesting implications for gauge coupling unification [69]. Since we are mainly interested in the effects of the intermediate scale rather than the effects of gauge symmetry breaking, we choose the above scheme due to its simplicity. For consistency, the pattern $M_{\text {cond }} \ll M_{I} \ll M_{\text {string }}$ is always assumed. Therefore, we shall integrate out the hidden-matter fields below $M_{I}$ and the effective lagrangian at $M_{\text {cond }}$ will consist of the moduli and the gauge composites only.

The superpotential for the hidden sector matter fields that we use is the following:

$$
W_{H M}=\frac{1}{2} \lambda^{i j} A \Phi_{i} \Phi_{j}+\frac{1}{3} \lambda^{\prime} A^{3} .
$$

It is worth remarking the curious fact that in all the examples of semirealistic superstring models with exactly three generations of matter that have been studied so far [82] no cubic self-coupling of gauge singlets seems to arise in the superpotential. However, there are indeed cubic couplings in the superpotential that involve two or three different gauge singlets $\left(\kappa_{\alpha \beta}\left(A^{\alpha}\right)^{2} A^{\beta}\right.$ or $\kappa_{\alpha \beta \gamma} A^{\alpha} A^{\beta} A^{\gamma}$ with $\alpha, \beta$, and $\gamma$ all different). The cubic selfcoupling is, however, not ruled out on any physical grounds. So, just to be consistent with the current literature, one should perhaps introduce at least a pair of gauge singlets, one of which is coupled to the gauge-charged matter fields. In that sense our case is a toy model describing the situation where the gauge singlets have mutual couplings comparable to our $\lambda^{\prime}$. However, for the general analysis of gaugino condensation in the presence of an intermediate scale, no new feature can be expected to arise from the extra gauge singlets as compared to our simplified case.

When constructing our model, two symmetry principles have been used to constrain the Lagrangian: First, the LEEFT must be T-modular invariant to all orders. Second, 
S-duality is a symmetry in the weak-coupling limit $\langle S+\bar{S}\rangle \rightarrow \infty$, as has been discussed in Section 5.3. Furthermore, we adopt also the point of view that the Kähler potential is renormalized instead of the gauge kinetic term when including the renormalization effects of the tree-level gauge coupling $S+\bar{S}$. This viewpoint is especially clear in the linear-multiplet formalism of the LEEFT. For example, in the linear-multiplet formalism, the cancellation of modular anomaly is achieved by adding the Green-Schwarz (GS) counterterm through the linear multiplet, which contains the string two-index antisymmetric tensor field. When going from the linear-multiplet formalism to the chiral formalism by performing the supersymmetric duality transformation, the GS counterterm of the linear-multiplet formalism transforms into the renormalization of the treelevel coupling $S+\bar{S}$ in the Kähler potential of the chiral formalism only. The gauge kinetic term of the corresponding chiral formalism remains unrenormalized [77]. Hence, we will include the renormalization and intermediate-scale threshold corrections only in the dilatonic part of the Kähler potential. It is worth noting that in the exact S-duality limit, in our chiral multiplet approach, the superpotentials for the matter field as well as the chiral condensate are absent. In constructing an effective theory for the chiral condensate field, consistent with the symmeteries of the underlying theory (modular and $S$ duality symmetries), we include the wave function renormalization of the condensate, $H$, in the Kähler potential. Put differently, the usual superpotential $W_{N P} \sim H^{3}$ is absent by requiring S-invariance in the $g \rightarrow 0$ limit; and so in the effective theory for this field, rather than having a quantum correction of the form $W_{q} \sim H^{3} \ln (H / \mu)$, we have a renormalization of the Kähler potential corresponding to the wave function renormalization of $H$.

Let us start with the construction of the Kähler potential. We derive the Kähler potential $K$ in two slightly different ways. The first derivation is straightforward: we take the canonically normalized mass of the fields $\Phi_{i}$ (which is a field-dependent, modularinvariant quantity) as the dynamically generated intermediate scale $M_{I}$, and the gauge coupling at the condensation scale is obtained easily by running the gauge coupling from the string scale first to the intermediate scale, and then to the condensation scale together with the fact that the matter fields of mass $M_{I}$ decouple below $M_{I}$.

In the second derivation, we apply the result derived in ref. [83] for the corrections to the gauge coupling at a field-theoretical threshold to one loop. Their result was derived for a generic supergravity model with a threshold scale, with no reference to modular 
invariance. In a modular invariant theory, we can show that both approaches result in the same gauge coupling, and therefore the same Kähler potential $K$.

The no scale case of the Kähler potential [67] (without matter fields, i.e., with pure $E_{8}$ gauge group) at the condensation scale is given by

$$
K=-\ln m_{0}-3 \ln \left(1-m_{0}^{1 / 3} Q\right)+G
$$

where,

$m_{0}=S+\bar{S}-b G+3 b \ln Q ; \quad G=-3 \ln (T+\bar{T}) ; \quad Q=|H|^{2} e^{G / 3} ; \quad-3 b=2 b_{0}=\frac{1}{8 \pi^{2}} C\left(E_{8}\right)$

Here, $M_{p l}=1$; and notice that the UV cut-off is taken to be $M_{\text {string }}=(S+\bar{S}-b G)^{-1 / 2}$ meaning that the condensation scale is really in these units, $Q /(S+\bar{S}-b G)$.

In the presence of an intermediate scale the renormalization of the gauge coupling in $m_{0}$ will be different from that of ref. [67]. If we include the threshold corrections at one loop, we get

$$
m_{0} \rightarrow m=S+\bar{S}-b G+3\left[b^{>} \ln \left(\frac{M_{I}^{2}}{M_{\text {string }}^{2}}\right)+b^{<} \ln \left(\frac{Q /(S+\bar{S}-b G)}{M_{I}^{2}}\right)\right],
$$

and the Kähler potential at the condensation scale is:

$$
K=-\ln m-3 \ln \left(1-m^{1 / 3} Q\right)+G
$$

Here, $b^{>}$and $b^{<}$are proportional to the $\beta$-function coefficients above and below the intermediate scale, respectively:

$$
b^{>}=\left(3 C_{G}-C_{M}\right) / 24 \pi^{2}, \quad b^{<}=C_{G} / 8 \pi^{2},
$$

where $C_{G}$ and $C_{M}$ are the quadratic Casimirs:

$$
C_{G}=T(a d j) ; \quad C_{M}=\sum_{r} n_{r} T(r) ; \quad T(r)=\operatorname{Tr}_{r}\left(T^{2}\right),
$$

with $r$ labelling the representations of the gauge group, and $n_{r}$ being the number of fields in the $r$ representation. As expected, in the absence of $M_{I}$, i.e., for $b^{>}=b^{<}$, we recover the Kähler potential of ref [67]. Let us briefly note that the general form of the Kähler potentials (3) and (6) is simply obtained by starting with the modular invariant tree level Kähler potential (supplied with the appropriate GS counter-term, $G$ ) which includes the kinetic term for the condensate field, $H$ :

$$
K=-\ln (S+\bar{S}-b G)-3 \ln \left(e^{-G / 3}-f(S, \bar{S})|H|^{2}\right),
$$


and imposing S-invariance, which gives $f=(S+\bar{S})^{1 / 3}$ up to an S-invariant factor. Finally one replaces $S+\bar{S}-b G$ with the one-loop renormalized effective coupling at the condensation scale, which we have denoted $m=2 / g_{\text {eff }}^{2}\left(M_{\text {cond }}\right)$.

The modular invariant scale $M_{I}$ has to be determined - it is the modular invariant, canonically normalized mass of $\Phi$, and not simply the vev of the gauge singlet $A$, which has a nonzero modular weight. Before computing $M_{I}$, let us make the distinction between the GS terms above and below the threshold, namely,

$$
\begin{aligned}
& G^{>}=-3 \ln \left(T+\bar{T}-|A|^{2}-\sum\left|\Phi_{i}\right|^{2}\right), \\
& G^{<}=-3 \ln \left(T+\bar{T}-|\langle A\rangle|^{2}\right) .
\end{aligned}
$$

Indeed, the difference arises only due to the change in the spectrum as the threshold is crossed. "We analyse the theory with all the massive fields $\left(\Phi_{i}\right.$ and $\left.A\right)$ "integrated out" at the condensation scale, so that in the first line of eq (5.28), these fields are replaced with their vacuum expectation values to obtain $G^{<}$in the second line. We discuss what kind of an approximation this replacement entails at the end of this section.

It is straight forward to show that the canonically normalized mass is:

$$
M_{I}^{2}=e^{K}\left(K^{\varphi \bar{\varphi}}\right)^{2}|\lambda A|^{2}=\frac{|\lambda A|^{2} e^{G / 3}}{9(s+\bar{s}-b G)}\left(1+\frac{b}{s+\bar{s}-b G}\right)^{-2} .
$$

Modular invariance is automatic due to the appropriate G-S terms, provided that $A$ has the following modular transformation property: $A \rightarrow|i \gamma T+\delta|^{-1} A$, i.e., has modular weight -1 .

We now derive the above Kähler potential by a different argument. It can be shown [83] that in the presence of a mass, in a YM + supergravity effective theory, the gauge couplings receive threshold correction at a scale $\Lambda_{I}$ given by

$$
\begin{aligned}
\frac{1}{g^{2}\left(p_{<}\right)}-\frac{1}{g^{2}\left(p_{>}\right)} & =b_{0}^{>} \ln \frac{p_{>}^{2}}{\Lambda_{I}^{2}}+b_{0}^{<} \ln \frac{\Lambda_{I}^{2}}{p_{<}^{2}}-\left(c^{>}-c^{<}\right) K \\
& -\frac{1}{8 \pi^{2}}\left(T(a d j)^{>}-T(a d j)^{<}\right) \ln g^{-2} \\
& +\frac{1}{8 \pi^{2}} \sum_{r} T(r) \ln \operatorname{det} Z_{\text {massive }}^{r}
\end{aligned}
$$

The group theoretic factors $c^{>}$and $c^{<}$are respectively given by:

$$
\begin{aligned}
c^{>} & =\left(-C_{G}+C_{M}\right) / 16 \pi^{2} ; \quad c^{<}=-C_{G} / 16 \pi^{2} ; \\
c^{>}-c^{<} & =b_{0}^{>}-b_{0}^{<}=-3 / 2\left(b^{>}-b^{<}\right)=C_{M} / 16 \pi^{2} .
\end{aligned}
$$


The Kähler function, wave function renormalization matrix $Z$ of the massive fields, and the (effective) coupling $g$ on the right hand side of the above formula are all tree level quantities at the intermediate scale. The derivation of the above equation assumes noncanonical normalization of the tree level kinetic terms in the supergravity Lagrangian. In particular, modular invariance plays no role, and the intermediate scale is not fixed by modular invariance and canonical normalization. Therefore, we take $\Lambda_{I}^{2}=|\lambda A|^{2}$ as one would in the noncanonical normalization. The Kähler term in eq. (5.30) must contain the contribution of the massive fields, i.e., it is $K_{\text {tree }}=-\ln (S+\bar{S}-b G)+G$, with $G$ given in the first line of eq. (5.28). For the UV cut-off, we use $M_{\text {string }}$ and for condensation scale $M_{\text {cond }}^{2}=Q /(S+\bar{S}-b G)$, as before. The normalization matrix for the $\Phi$ fields is given simply by the Kähler metric components $K_{I \bar{J}}^{>}$. One only finds contributions from the diagonal blocks: $Z_{I \bar{I}}=3[1+b /(S+\vec{S}-b G)] e^{G / 3}$. Hence,

$$
\begin{aligned}
2 \sum_{r} T(r) \ln \operatorname{det} Z_{\text {massive }}^{r} & =2 \sum_{r} T(r) \sum_{I} \ln \left[3 e^{G / 3}\left(1+\frac{b}{S+\bar{S}-b G}\right)\right]_{r} \\
& =\sum_{r} T(r) n_{r} \ln \left[3\left(1+\frac{b}{S+\bar{S}-b G}\right) e^{G / 3}\right]^{2} \\
& \left.=\left(b_{0}^{>}-b_{0}^{<}\right) \ln \left[3\left(1+\frac{b}{S+\bar{S}-b G}\right)\right) e^{G / 3}\right]^{2},
\end{aligned}
$$

Finally, notice that in our scheme of generating the intermediate mass the $T(a d j)^{>}$ $T(a d j)^{<}=0$, and thus the corresponding term in the threshold correction will be absent. Making the above replacements in eq. (5.30) gives:

$$
\begin{aligned}
S+\bar{S}-b G & \rightarrow S+\bar{S}-b G+2\left[-b_{0}^{>} \ln \left(\frac{|\lambda A|^{2}}{M_{\text {string }}^{2}}\right)-b_{0}^{<} \ln \left(\frac{Q /(S+\bar{S}-b G)}{|\lambda A|^{2}}\right)\right] \\
& -\left(b_{0}^{>}-b_{0}^{<}\right)\left[G-\ln (S+\bar{S}-b G)-\ln \left(\frac{e^{G / 3}}{9\left(1+\frac{b}{S+\bar{S}-b G}\right)^{2}}\right)\right] \\
S+\bar{S}-b G & +3 b^{>} \ln \left(\frac{|\lambda A|^{2} e^{G / 3}\left(1+\frac{b}{S+\bar{S}-b G}\right)^{-2}}{9(S+\bar{S}-b G) M_{\text {string }}^{2}}\right) \\
& +3 b^{<} \ln \left(\frac{Q}{|\lambda A|^{2} e^{G / 3}\left(1+\frac{b}{S+\bar{S}-b G}\right)^{-2} / 9}\right)
\end{aligned}
$$

which is precisely the same as in eq. (5.24). To summarize, our Kähler potential is given by eq. (5.25) and (5.24) which is the extension of that proposed in ref. [67]. This extension consisted of the renormalization of the gauge coupling in $K$, including the one-loop field-theoretical threshold corrections around $M_{I}$, the modular invariant, canonically normalized intermediate mass scale. 
A comment on integrating out the heavy fields and replacing them with their vevs is perhaps in order. We have obtained the renormalized Kähler function at the condensation scale. Since the masses of the heavy fields $\left(\mathcal{O}\left(M_{I}\right)\right)$ are, by assumption, much larger than the condensation scale, we must integrate out all the heavy fields. We assume that the gauge-singlet $A$ is heavy, with $M_{A} \sim \mathcal{O}\left(M_{I}\right) \gg M_{\text {cond }}$; i.e., the self coupling of $A$ in the superpotential $W(A)=\frac{\lambda^{\prime}}{3} A^{3}$ is sufficiently large. Then it is easy to show that if we integrate out the fields $A$ and $\Phi^{i}$ at tree level, the following terms are generated in the effective potential:

$$
\begin{aligned}
V_{e f f} & =M_{A}^{-2} K^{a \bar{a}} \mid\left[\left(K_{i \bar{\jmath} a} K_{\ell \bar{m} \bar{a}}\right)\left|\partial_{\mu} z^{i} \partial^{\mu} \bar{z}^{\bar{\jmath}} \partial_{\nu} z^{\ell} \partial^{\nu} \bar{z}^{\bar{m}}+\left(V_{a} V_{\ddot{a}}\right)\right|-\left(\left(V_{a} K_{i \bar{\jmath} \bar{a}}\right) \mid \partial_{\mu} z^{i} \partial^{\mu} \bar{z}^{\bar{\jmath}}+h . c .\right)\right] \\
& =\left(M_{A}^{-2} K^{a \bar{a}} K_{i \bar{\jmath} a} K_{\ell \bar{m} \bar{a}}\right) \mid \partial_{\mu} z^{i} \partial^{\mu} \bar{z}^{\bar{\jmath}} \partial_{\nu} z^{\ell} \partial^{\nu} \bar{z}^{\bar{m}}
\end{aligned}
$$

The quantities denoted by a vertical bar are evaluated at the vacuum $\left(a=\langle a\rangle, \varphi^{i}=\right.$ $\left.\left\langle\varphi^{i}\right\rangle=0\right)$. The last line follows from the fact that $V_{a}=\partial V / \partial a$ vanishes at $\langle A\rangle$. Since, the effective potential (5.34) that arises contains only 4-derivative couplings, at energies well below $M_{A}$, i.e., at the condensation scale it can be ignored, and in our analysis, we can replace the heavy fields with their vev's.

We close this with the following remarks. We notice that a constant term is generated in the superpotential, namely

$$
c=\frac{\lambda^{\prime}}{3}\langle A\rangle^{3}
$$

In essentially all models of gaugino condensation, introduction of a constant superpotential is necessary for breaking supersymmetry. However, the constant is usually either introduced in an ad hoc way, or its origin is from compactification of superstrings. Namely, the vev of the compactified components of the 3-form, $H_{l m n}$ from 10-D supergravity [74]. In the latter case, the constant has the undesirable property that it is of the order of Planck mass (thus breaking supersymmetry at $M_{P l}$ ) and that it is quantized, presumably in units of $M_{P l}$. The above constant $c$ is clearly much smaller (of the order of $M_{I}$ ) and it is continuous. The second remark has to do with the fact that we know [see eq. (5.28)] that $|\langle A\rangle|^{2}<\langle T+\bar{T}\rangle$. Further, we know that the vev of $T$ is not determined perturbatively. The nonperturbative superpotential for the condensate is what will eventually allow us to fix $\langle T\rangle$. So, how are we justified in integrating out $A$ but not $T$ ? The only justification we offer is the fact that the $T$ modulus remains massless to all orders in perturbation theory until supersymmetry is broken (nonperturbatively) by the gaugino condensation (or otherwise), whereas $A$ is by construction massive $\left(M_{A} \sim M_{I}\right)$. 


\subsection{Scalar Potential and the Vacuum}

The dynamical fields at the condensation scale in our model are $S, H$, and $T$. The scalar potential is given by:

$$
V=e^{K}\left[K^{i \bar{\jmath}}\left(K_{i} W+W_{i}\right)\left(K_{\bar{\jmath}} \bar{W}+\bar{W}_{\bar{\jmath}}\right)-3|W|^{2}\right]
$$

and the Kähler metric written in terms of $m=2 / g_{\text {eff }}^{2}\left(M_{\text {cond }}\right)$ [eq. (5.24)], $Q=|H|^{2} e^{G / 3}$, and their derivatives with respect to the scalar fields is given by:

$$
\begin{aligned}
K_{i \bar{\jmath}}=m^{-2}\left\{m_{i} m_{\bar{\jmath}} \tilde{x}\right. & +m(\xi-1) m_{i \bar{\jmath}}+\left(\xi+\xi^{2}\right)\left(m_{i} q_{\bar{j}}+m_{\bar{\jmath}} q_{i}\right) \\
& \left.+3 m^{2}\left[\xi q_{i \bar{\jmath}}+\left(\xi+\xi^{2}\right) q_{i} q_{\bar{j}}\right]+m^{2} G_{i \bar{\jmath}}\right\}
\end{aligned}
$$

where

$$
x=m^{1 / 3} Q, \quad \xi=\frac{x}{1-x}, \quad \tilde{x}=1-2 \xi / 3+\xi^{2} / 3,
$$

and

$$
m_{i}=\partial_{i} m, \quad q=\ln Q, \quad q_{i}=\partial_{i} \ln Q, \quad \text { etc. }
$$

Notice that $G_{i \bar{j}}=0$ unless $i=j=t, m_{h \bar{\jmath}}=0$, and $q_{s}=0$. The nonperturbative part of the superpotential is of the form

$$
W_{N P}=\alpha e^{-S / b} Y^{n}\left(\ln \frac{Y}{\mu}\right)^{k}, \quad Y=H e^{S / 3 b<}
$$

with $n<3$ (the Veneziano-Yankielowicz superpotential is the special case of $n=3$ and $k=1$ ). The reason the exponents $n$ and $k$ are introduced is because, as stressed earlier, it is the Kähler potential (5.25) that includes the gaugino condensate wave function renormalization, while the superpotential vanishes in the weak coupling limit.

Is the potential positive semi-definite? Numerical analysis indicates that the answer is yes. Analytically, this would be obvious if $\langle W\rangle$ could be shown to be zero. In fact, numerically ${ }^{3}$ we find that at the minimum of $V$,

- $\langle W\rangle \simeq 0$.

- $m=2 / g_{\text {eff }}^{2}\left(M_{\text {cond }}\right) \rightarrow 0$.

To see that $\langle W\rangle=0$ at $V=0$, guided by the second numerical result above, we expand $V$ in powers of $m$ in the limit $m \rightarrow 0$. A lengthy but straightforward calculation shows that when $\langle W\rangle=0, V \sim \mathcal{O}(m)+$ higher order as $m \rightarrow 0$; and for $\langle W\rangle \neq 0$,

\footnotetext{
${ }^{3}$ In the numerical analysis, the value of $\langle$ Ret $\rangle$ was fixed and $s$ and $h$ were varied (see later).
} 


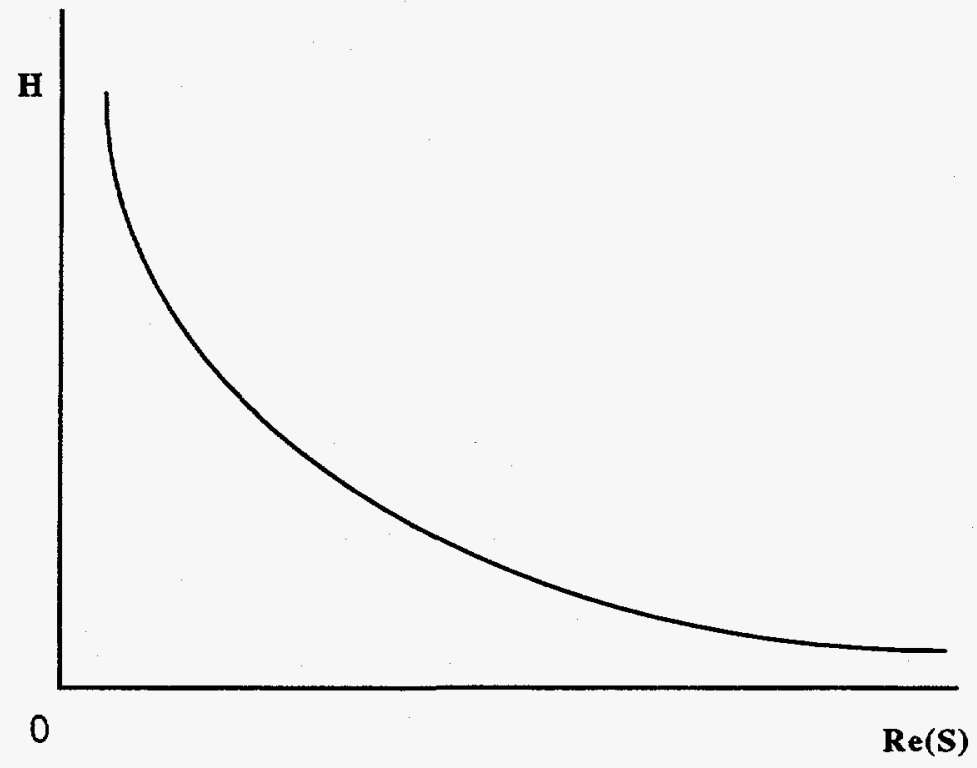

Figure 5.1: The boundary between the kinematically forbidden (below the curve) and allowed regimes contains the nontrivial minimum of the scalar potential $V(s, h)$.

there would be a pole $\sim 1 / m$ in $V$ (this is because the threshold corrections at $M_{I}$ cause the Kähler potential to be no longer exactly 'no-scale'). No such pole was found; the minimum of $V(s, h)$ corresponds to the minimum of $m(s, h)$ (which is zero). The analytical asymptotic expansion of $V$ in $m$, and the numerical results are compatible only for $\langle W\rangle=0$. The reality of the Kähler function, and the hierarchy $M_{\text {cond }} \ll M_{I} \ll M_{p l}$ restrict the kinematically allowed region of the parameter space such that: ${ }^{4}$

$$
a<\sqrt{2 t}, \quad \lambda a / 3 \gg h
$$

(for simplicity, we take both $s$ and $h$ to be real). The kinematically forbidden and allowed regions are typically separated as shown in Fig. 1. The boundary between the two regions contains the nontrivial minimum satisfying $m=0$ and $\langle W\rangle=0$ (as well as the trivial minimum $(s, h)=(\infty, 0))$.

Both $m$ and $\langle W\rangle$ increase monotonically from zero in both $h$ and Res near the vacuum ${ }^{5}$. The plot shown in Fig. 2 shows that $V(s, h)$ also monotonically increases in both directions, and particularly sharply in the $h$ (condensate) direction, indicating

\footnotetext{
${ }^{4}$ Hereafter, lower case letters indicate the scalar components of the corresponding superfields.

'Vacuum' here refers to the nontrivial minimum of the potential.
} 
confinement. In the direction of the dilaton, the potential increases quadratically as a function of $S$. This can be seen by looking at the $S$-dependence of $V(m \approx 0)$. Furthermore, we notice that the dilaton does 'run away', but in the correct direction! Namely, to some finite value of $s$ (which separates the kinematically allowed and forbidden regions, at the nontrivial minimum of the potential). This is in addition to the usual runaway behaviour to $s \rightarrow \infty$, which is the susy-restoring and deconfining limit. Also interesting is the behaviour of $m=2 / g_{\text {eff }}^{2}\left(M_{\text {cond }}\right)$ near the vacuum, which as noted above, is $m \rightarrow 0$ or $g_{\text {eff }}\left(M_{\text {cond }}\right) \rightarrow \infty$ (while $g_{s t}$ remains finite). This is exactly what one expects physically, since the condensate - the bound state in the strong coupling regime - is expected to correspond to a stable vacuum solution. Notice, however, that the relations $\langle V\rangle=\langle W\rangle=0$ imply that supersymmetry remains unbroken.

So far, the role of the intermediate scale has been masked. In the following, we show that in the effective theory that we are considering, the free parameter $\mu$ in the nonperturbative superpotential (5.38) is intimately related to the intermediate mass. Furthermore, we shall see that the intermediate mass plays a role in allowing a sensible hierarchy between the Planck scale and condensation scale, consistent with the phenomenologically acceptable values of $\langle\operatorname{Res}\rangle$ and $\langle\operatorname{Re} t\rangle$. For this, we shall give a rough argument below. Of course, the obvious effect that can immediately be associated with the intermediate mass is the shift it causes in the condensation scale, since in its presence the gauge coupling runs differently, as discussed in Section 5.5.

In the presence $M_{I}$, the vacuum is characterized by two independent conditions:

$$
m=0, \quad\langle W\rangle=0 \text {. }
$$

These two conditions together imply that:

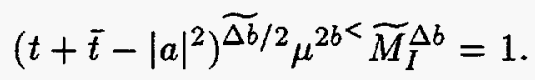

Here, $\Delta b=b^{>}-b^{<}$and $\widetilde{\Delta b}=b-b^{<}$. This can be re-written as follows:

$$
s+\bar{s}-b G=b\left[\frac{|\lambda a|}{3} \mu^{b^{<} / \Delta b}\left(t+\bar{t}-|a|^{2}\right)^{\widetilde{\Delta b} / 2 \Delta b}-1\right]^{-1} .
$$

This equation should be viewed as a relation between $s, t$, and $\mu$ in the vacuum of the theory. Is this compatible with phenomenologically acceptable values, $\langle s+\bar{s}\rangle \sim \mathcal{O}(1)$ and $\langle t+\bar{t}\rangle \sim \mathcal{O}(1)$ ? If in eq. (5.41) we set $\left\langle t+\bar{t}-|a|^{2}\right\rangle \simeq 1,{ }^{6}$ which is also the assumption

\footnotetext{
${ }^{6}$ Notice that this does not restrict $M_{I}$ since $\lambda$ can be chosen small enough to give the assumed hierarchy $M_{I} \ll M_{P l}$.
} 


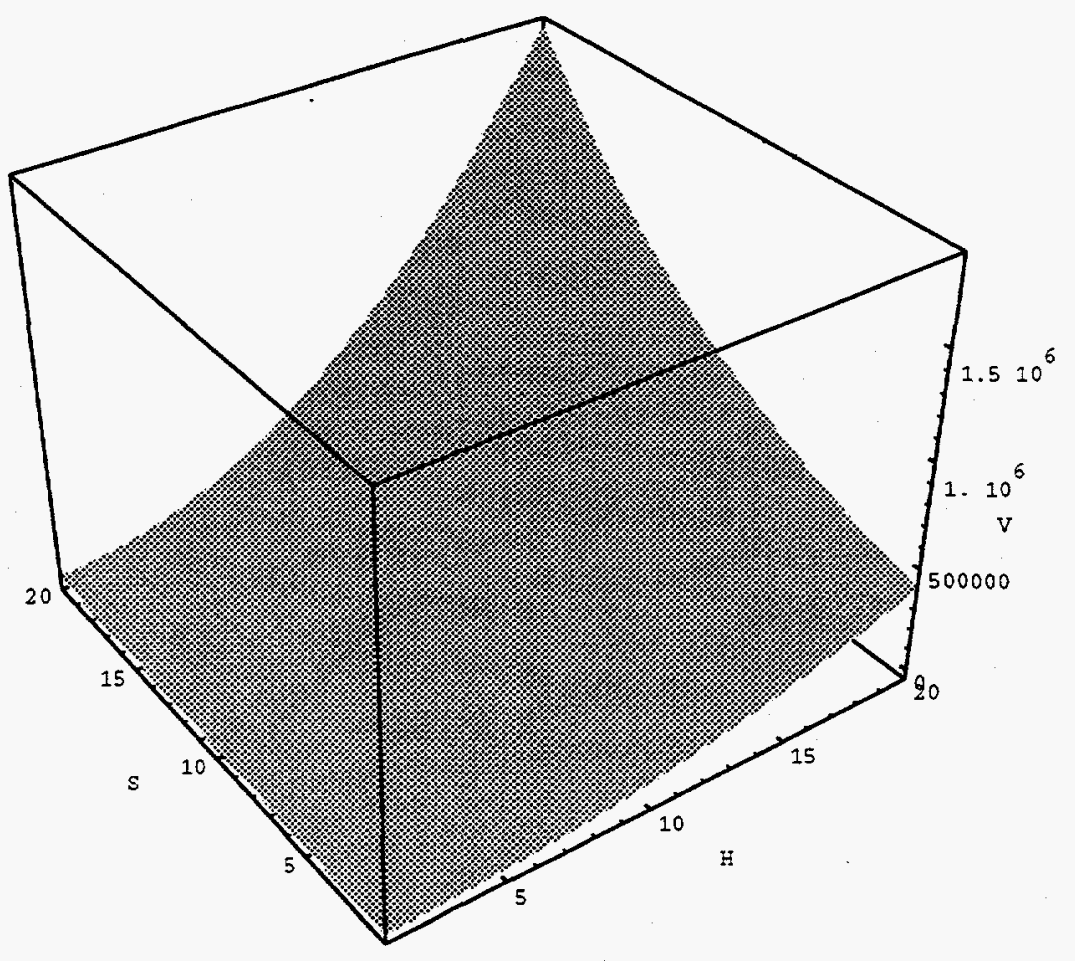

Figure 5.2: The scalar potential $V(s, h)$. The graph corresponds to the example of $S U(3)$ as the gauge group, and assuming $(n, k)=(2,1),\langle t\rangle=1$ for the internal modulus, $\langle a\rangle=1.1, \lambda=0.1$, and $\lambda^{\prime}=1$. The kinematically forbidden region of the $h-s$ plane has been excluded here; i.e., the minimum of this plot is a point on the curve illustrated in Fig. 1 , in this case $(\operatorname{Re} s, h)=(2.66,0.00044)$. 


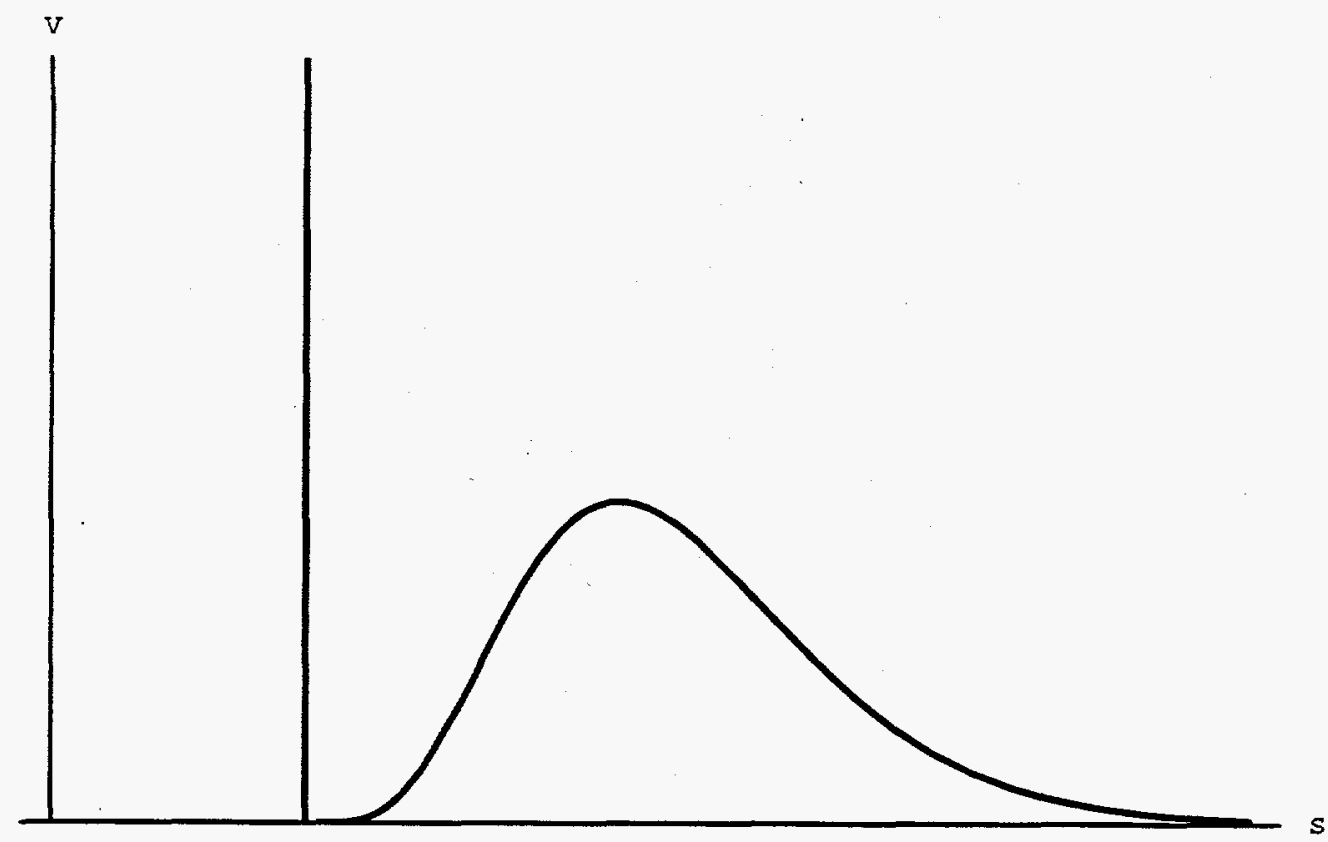

Figure 5.3: The runaway behaviour of the dilaton in both directions. In the left direction the minimum corresponds to the effective coupling becoming strong. There, the potential 'runs' into the kinematically forbidden region. 
in the numerical analysis, then it is easy to see that in order to get $\langle s+\bar{s}\rangle \sim \mathcal{O}(1)$,

$$
\frac{\lambda\langle a\rangle}{3} \mu^{-|b</ \Delta b|}-1
$$

should be $\mathcal{O}(1)$. That is to say, for $b^{</ \Delta b}$ of order unity,

$$
\mu \sim \lambda\langle a\rangle \sim \mathcal{O}\left(\widetilde{M_{I}}\right)
$$

The free parameter of the effective superpotential for the condensate is, therefore, 'locked' to the intermediate mass. This rough argument also shows that, with mild $(\mathcal{O}(1))$ fine tuning, it is at least possible in this scheme to obtain a phenomenologically acceptable value for the dilaton, and at the same time achieve condensation and generate the desired hierarchy. ${ }^{7}$ To see this, consider eq. (5.40) again which together with eq. (5.43) tells us that:

$$
|\langle h\rangle| \sim \exp \left(\frac{-\langle s+\bar{s}\rangle}{6 b^{<}}\right) \mu \sim \exp \left(\frac{-\langle s+\bar{s}\rangle}{6 b^{<}}\right) \widetilde{M_{I}} .
$$

Again, we see that the parameters, which are admittedly model-dependent but are nevertheless, dictated by the presence of the intermediate mass and the choice of the gauge group can allow for a condensate whose vev is suppressed compared to the parameter $\mu$ which by requirement of phenomenology is of the order of the intermediate mass.

\subsection{Conclusions}

Perhaps the most peculiar feature of the model of gaugino condensation that we have discussed above is the running behaviour of the dilaton, which is schematically shown in Fig. 3. The finite value of $\operatorname{Re} S$ that the potential "runs" to is, as noted earlier, on the boundary of the kinematically forbidden region, and this value corresponds precisely to $1 / g_{\text {eff }}\left(M_{\text {cond }}\right) \rightarrow 0$. We interpret this running of $\operatorname{Re} S$ in both directions as a manifestation of the approximate S-duality which constrains the Kähler potential which we have started with - the behaviour of the strong and weak coupling (small and large $S$, respectively) regimes are alike. The intermediate scale serves basically to shift the renormalization running of the gauge coupling and allow for a hierarchy between the unification and condensation scale by shifting the condensation scale and/or the

\footnotetext{
${ }^{7}$ We hesitate to call this stabilization of the dilaton because the finite value of $\langle\operatorname{Re} S\rangle$ at which the potential runs to a minimum is at the boundary of the kinematically forbidden regime; $V$ is not smooth there.
} 
unification scale (see ref. [69] for detailed discussion of the latter). The intermediate mass (or rather, the vev of the gauge singlet) was assumed, but of course a realistic model should dynamically generate such an $M_{I}$ consistent with phenomenology as discussed near the end of the previous section, and thereby giving a phenomenologically correct hierarchy of scales. This is of course a more significant issue in the models where supersymmetry is broken by gaugino condensation at the scale $M_{\text {cond }}$.

However, as we have seen, neither S-duality nor the 1-loop corrections to the dilaton in $K$ (including the dilaton dependent threshold corrections at $M_{I}$ are enough to break supersymmetry in such models. If one is to include any perturbative (1-loop) corrections to $K$, results such as those presented here or in ref. [67] seem to indicate that it is more meaningful to include the full renormalization of the Kähler potential, and all other terms that arise at 1-loop in the supergravity and super-YM effective action which are relevant to gaugino condensation, such as

$$
\int d^{4} \theta E\left(\frac{N_{G} \ln \Lambda^{2}}{32 \pi^{2}}\right)(R e S)^{2}\left|W^{\alpha} W_{\alpha}\right|^{2}
$$

These have been calculated as discussed in previous chapters, and work along this direction is under progress elsewhere [85]. Indeed, as it has been argued by Banks and Dine, if stabilization of the dilaton (and other moduli) and supersymmetry breaking are really one and the same phenomena, as they appear to be, then stringy nonperturbative corrections to Kähler potential are crucial and should be included [86]. A realization of this proposal in the context of linear multiplet formulation of gaugino condensate appears in ref [87]. Of course, the exact form of these nonperturbative corrections are not yet understood. But one can perhaps expect that the recent developments in string dualities can shed some light on the latter, and on the stabilization of string moduli and supersymmetry breaking. 


\section{Appendix}

\section{A. Relations among operators}

In this appendix we derive relations among the various operators that appear in the traces needed to evaluate the one-loop effective action. We adopt the gauge sign conventions of [42], [62]:

$$
\begin{aligned}
\mathcal{D}_{\mu} & =\nabla_{\mu}+i A_{\mu}, \quad A_{\mu}=T_{a} A_{\mu}^{a}, \quad T_{a \bar{j}}^{\bar{i}}=\left(T_{a j}^{i}\right)^{*}, \\
\mathcal{D}_{\mu} z^{i} & =\partial_{\mu} z^{i}+i A_{\mu}^{a}\left(T_{a} z\right)^{i}, \quad \mathcal{D}_{\mu} \bar{z}^{\bar{m}}=\partial_{\mu} \bar{z}^{\bar{m}}-i A_{\mu}^{a}\left(T_{a} \bar{z}\right)^{\bar{m}}, \\
F_{\mu \nu} & =\frac{1}{i}\left[\mathcal{D}_{\mu}, \mathcal{D}_{\nu}\right]=\nabla_{\mu} A_{\nu}-\nabla_{\nu} A_{\mu}+i\left[A_{\mu}, A_{\nu}\right] \\
F_{\mu \nu}^{a} & =\nabla_{\mu} A_{\nu}^{a}-\nabla_{\nu} A_{\mu}^{a}-c^{a}{ }_{b c} A_{\mu}^{b} A_{\nu}^{c} .
\end{aligned}
$$

Our other conventions and notations are given in Section 3.3.

We first consider constraints on covariant scalar derivatives that follow from gauge invariance. We define

$$
\begin{aligned}
\mathcal{K}_{a b} & =\frac{1}{x} K_{\bar{m} j}\left(T_{a} \bar{z}\right)^{\bar{m}}\left(T_{b} z\right)^{j}, \quad \mathcal{D}_{a}=K_{i}\left(T_{a} z\right)^{i}, \quad \mathcal{D}=\frac{1}{2 x} \mathcal{D}_{a} \mathcal{D}^{a}, \\
f_{a b}(z) & =\delta_{a b} f(z), \quad f=x+i y .
\end{aligned}
$$

The classical scalar potential is $\hat{V}+\mathcal{D}$. It follows from the gauge invariance of the Kähler potential $K$ that:

$$
\begin{gathered}
\delta_{a} K=K_{i}\left(T_{a} z\right)^{i}-K_{\bar{m}}\left(T_{a} \bar{z}\right)^{\bar{m}}=0, \quad D_{i} D_{j} \mathcal{D}_{a}=D_{\bar{m}} D_{\bar{n}} \mathcal{D}_{a}=0 \\
K_{i \bar{m}} D_{\bar{n}}\left(T_{a} \bar{z}\right)^{\bar{m}}=K_{j \bar{n}} D_{i}\left(T_{a} z\right)^{j}, \quad D^{i}\left(T_{a} \bar{z}\right)^{\bar{m}}=D^{\bar{m}}\left(T_{a} z\right)^{i} \\
K_{i j}\left(T_{a} z\right)^{j}+K_{j}\left(T_{a}\right)_{i}^{j}=K_{i \bar{m}}\left(T_{a} \bar{z}\right)^{\bar{m}}, \quad D_{k} D_{j}\left(T_{a} z\right)^{i}=-R_{j \bar{m} k}^{i}\left(T_{a} \bar{z}\right)^{\bar{m}}
\end{gathered}
$$

where $K_{i j}=\partial_{i} \partial_{j} K=\partial_{i} K_{j}$, and the second and third lines follow from the first by taking successive scalar derivatives. Here $\partial_{I}=\partial / \partial z^{I}, I=i, \bar{\imath}, D_{I}$ is the reparameterization covariant scalar derivative, and $R_{i \bar{m} j \tilde{n}}$ is the Kähler curvature tensor. Indices are lowered and raised, respectively, with the Kähler metric $K_{i \bar{m}}$ and its inverse $K^{i \bar{m}}$. Similarly, it follows from the gauge invariance of $f$ that

$$
\begin{aligned}
\delta_{a} f & =f_{i}\left(T_{a} z\right)^{i}=0, \\
f_{i j}\left(T_{a} z\right)^{j} \bar{f}^{i} & =-f_{i} D_{j}\left(T_{a} z\right)^{i} \bar{f}^{j}, \quad f_{i j}\left(T_{a} z\right)^{j}\left(T_{b} z\right)^{i}=-f_{i}\left(T_{a} z\right)^{j} D_{j}\left(T_{b} z\right)^{i}, \\
f_{i} \bar{f}_{\bar{m}} D^{i}\left(T_{a} \bar{z}\right)^{\bar{m}} & =-f^{\bar{n}} \bar{f}_{\bar{n} \bar{m}}\left(T_{a} \bar{z}\right)^{\bar{m}}=-\bar{f}^{i} f_{i j}\left(T_{a} z\right)^{j}, \quad f_{i j}=D_{i} D_{j} f
\end{aligned}
$$


and from the gauge invariance of the superpotential $W$ that

$$
\begin{gathered}
A_{i}\left(T_{a} z\right)^{i}=A_{\bar{m}}\left(T_{a} \bar{z}\right)^{\bar{m}}=\mathcal{D}_{a} A, \\
A_{i j}\left(T_{a} z\right)^{i}+A_{i} D_{j}\left(T_{a} z\right)^{i}=\mathcal{D}_{a} A_{j}+K_{j \bar{m}}\left(T_{a} \bar{z}\right)^{\bar{m}} A, \\
A_{i j k}\left(T_{a} z\right)^{i}+A_{i j} D_{k}\left(T_{a} z\right)^{i}+A_{i k} D_{j}\left(T_{a} z\right)^{i}+A_{i} D_{k} D_{j}\left(T_{a} z\right)^{i} \\
=\mathcal{D}_{a} A_{j k}+K_{j \bar{m}}\left(T_{a} \bar{z}\right)^{\bar{m}} A_{k}+K_{k \bar{m}}\left(T_{a} \bar{z}\right)^{\bar{m}} A_{j} .
\end{gathered}
$$

The tensors $A_{i_{1} \ldots i_{n}}$ are reparameterization invariant covariant derivatives [30] of $A=$ $e^{K} W$. Using (A.3) and the definitions (A.2) we obtain

$$
\mathcal{K}_{a b}-\mathcal{K}_{b a}=\frac{i}{x} c_{a b c} \mathcal{D}^{c}, \quad \mathcal{K}^{a b}\left(\mathcal{K}_{a b}-\mathcal{K}_{b a}\right)=-\frac{1}{2 x^{2}} C_{G}^{(a)} \mathcal{D}_{a} \mathcal{D}^{a}
$$

where $C_{G}^{(a)}$ is the Casimir in the adjoint representation, $c_{a b c}$ are the structure constants of the gauge group, and

$$
\begin{gathered}
\left(T_{b} z\right)^{i} D_{i}\left(T_{a} z\right)^{j}=\left(T_{a} z\right)^{i} D_{i}\left(T_{b} z\right)^{j}+i c_{a b c}\left(T^{c} z\right)^{j} \\
\mathcal{D}_{b} K_{\bar{m} j}\left(T_{a} \bar{z}\right)^{\bar{m}}\left(T^{a} z\right)^{i} D_{i}\left(T^{b} z\right)^{j}=\mathcal{D}_{b} K_{\bar{m} j}\left(T_{a} \bar{z}\right)^{\bar{m}}\left(T^{b} z\right)^{i} D_{i}\left(T^{a} z\right)^{j}-\frac{1}{2} C_{G}^{(a)} \mathcal{D}_{a} \mathcal{D}^{a}
\end{gathered}
$$

Combining (A.3) and (A.5) we obtain

$$
\begin{aligned}
& A_{i} D^{i}\left(T_{a} \bar{z}\right)^{\bar{m}}= A_{i} D^{\bar{m}}\left(T_{a} z\right)^{i}=-A_{i}^{\bar{m}}\left(T_{a} z\right)^{i}+A^{\bar{m}} \mathcal{D}_{a}+A\left(T_{a} \bar{z}\right)^{\bar{m}} \\
& \bar{A}^{i} D_{i}\left(T_{a} z\right)^{k}= \bar{A}_{\bar{n}} D^{\bar{n}}\left(T_{a} z\right)^{k}=\bar{A}_{\bar{n}} D^{k}\left(T_{a} \bar{z}\right)^{\bar{n}}=-\bar{A}_{\bar{n}}^{k}\left(T_{a} \bar{z}\right)^{\bar{n}}+\bar{A}^{k} \mathcal{D}_{a}+\bar{A}\left(T_{a} z\right)^{k} \\
& \mathcal{D}^{a} \bar{A}^{j k} A_{i j} D_{k}\left(T_{a} z\right)^{i}=-\frac{1}{2} \bar{A}^{j k} A_{i j k}\left(T_{a} z\right)^{i} \mathcal{D}^{a} \\
& \\
&+\frac{1}{2} R_{j \bar{m} k}^{i} \bar{A}^{j k} A_{i} \mathcal{D}^{a}\left(T_{a} \bar{z}\right)^{\bar{m}}+x \mathcal{D} A_{i j} \bar{A}^{i j}+\mathcal{D}^{a}\left(T_{a} \bar{z}\right)^{\bar{m}} \bar{A}_{\bar{m}}^{i} A_{i}
\end{aligned}
$$

To evaluate the one-loop effective action, we find it convenient to introduce the scalar field reparameterization covariant derivatives of the variable $\rho$, defined as the squared gauge coupling:

$$
\begin{aligned}
\rho=\frac{1}{x} & =g^{2}, \quad \rho_{i}=D_{i} \rho=-\frac{f_{i}}{2 x^{2}}, \quad \rho^{i}=K^{i \bar{m}} D_{\bar{m}} \rho=K^{i \bar{m}} \rho_{\bar{m}}, \\
\rho_{i j} & =D_{i} D_{j} \rho=-\frac{1}{2 x^{2}}\left(f_{i j}-\frac{f_{i} f_{j}}{x}\right) \\
D_{\bar{m} \rho_{i}}=\rho_{\bar{m} i} & =-\frac{1}{x} \bar{f}_{\bar{m}} \rho_{i}=2 x \rho_{\bar{m}} \rho_{i}, \\
D_{j}\left(x^{2} \rho_{i} \rho^{i}\right) & =x^{2} \rho^{i} \rho_{i j}, \quad D_{\bar{m}}\left(x^{2} \rho_{i} \rho^{i}\right)=x^{2} \rho_{\bar{m}}^{i} \rho_{i}, \\
D_{j} D_{k}\left(x^{2} \rho_{i} \rho^{i}\right) & =x^{2} \rho^{i} \rho_{i j k}, \quad \text { etc. }, \\
f_{\bar{m} i j}=R_{i \bar{m} j}^{k} f_{k} & =-2 x^{2} \rho_{\bar{m} i j}-2 x \bar{f}_{\bar{m}} \rho_{i j}-\frac{f_{i} f_{j} \bar{f}_{\bar{m}}}{2 x^{2}} .
\end{aligned}
$$


It follows from $\left[D_{\bar{m}}, D_{i}\right]\left(x^{2} \rho_{i} \rho^{i}\right)=0$ that

$$
\bar{f}^{k} \rho_{k i}^{j}+\frac{1}{x} \bar{f}^{k} \bar{f}^{j} \rho_{k i}=f_{k} \rho_{i}{ }^{k j}+\frac{1}{x} f_{k} f_{i} \rho^{k j} .
$$

In addition we introduce the variable

$$
a=A+\frac{\bar{f}^{i}}{2 x} A_{i}=e^{K / 2}\left(\bar{m}_{\psi}-\bar{m}_{\lambda}\right), \quad a_{i_{1} \cdots i_{n}}=D_{i_{1}} \cdots D_{i_{n}} a .
$$

The variables $a, \rho_{i j}$ and $1-x^{2} \rho^{i} \rho_{i}$, and all covariant derivatives thereof, vanish for effective supergravity theories obtained from superstrings in the classical limit: $f(z)=$ $s, K=-\ln (s+\bar{s})+G(z, \bar{z} \neq s, \bar{s}), W_{s}=0$.

We will also need the following identities involving the Yang-Mills field strength and the space-time curvature. It follows from manipulating products of the antisymmetric tensor $\epsilon_{\mu \nu \rho \sigma}$ that

$$
\begin{aligned}
\widetilde{M}_{1}^{\mu \nu} M_{\mu \rho}^{2} & =\frac{1}{2} g_{\rho}^{\nu} M_{1}^{\mu \sigma} \widetilde{M}_{\mu \sigma}^{2}-M_{\mu \rho}^{2} \widetilde{M}_{2}^{\mu \nu}, \quad \widetilde{M}_{\mu \nu}^{i}=\frac{1}{2} \epsilon_{\mu \nu \rho \sigma} M_{i}^{\rho \sigma}, \\
\tilde{F}_{\mu \nu}^{a} F_{b}^{\mu \nu} \tilde{F}_{\rho \sigma}^{b} F_{a}^{\rho \sigma} & =-\left(F_{\mu \nu}^{a} F_{b}^{\mu \nu}\right)^{2}-\left(F_{\mu \nu}^{a} F_{a}^{\mu \nu}\right)^{2}+4 F_{\rho \mu}^{a} F_{a}^{\rho \nu} F_{b}^{\sigma \mu} F_{\sigma \nu}^{b}, \\
\left(\tilde{F}_{\mu \nu}^{a} F_{a}^{\mu \nu}\right)^{2} & =-2\left(F_{\mu \nu}^{a} F_{b}^{\mu \nu}\right)^{2}+4 F_{\mu \nu}^{a} F_{a \rho \sigma} F^{b \mu \rho} F_{b}^{\nu \sigma}
\end{aligned}
$$

where $M_{\mu \nu}^{i}$ is any antisymmetric tensor-valued operator. Using the first of these gives

$$
\begin{aligned}
\operatorname{Tr} A^{\mu \nu} B_{\mu \rho} \tilde{C}_{\nu \sigma} D^{\sigma \rho} & =\frac{1}{4} \operatorname{Tr}\left[(\tilde{D} \cdot A)(B \cdot C)-(A \cdot B)(\tilde{C} \cdot D)-\tilde{A}^{\mu \nu} B_{\rho \sigma} C_{\mu \nu} D^{\rho \sigma}\right], \\
\operatorname{Tr} A^{\mu \nu} B_{\mu \rho} \tilde{C}^{\sigma \rho} D_{\sigma \nu} & =\frac{1}{4} \operatorname{Tr}\left[(A \cdot B)(\tilde{C} \cdot D)+(D \cdot A)(\tilde{B} \cdot C)-A^{\mu \nu} \tilde{B}_{\rho \sigma} C_{\mu \nu} D^{\rho \sigma}\right], \\
\operatorname{Tr} A^{\nu \sigma} B^{\rho \mu} \tilde{C}_{\mu \nu} D_{\rho \sigma} & =\frac{1}{4} \operatorname{Tr}\left[(\tilde{A} \cdot B)(C \cdot D)-(D \cdot A)(\tilde{B} \cdot C)-\tilde{A}^{\mu \nu} B_{\rho \sigma} C_{\mu \nu} D^{\rho \sigma}\right], \\
\tilde{F}_{\mu \nu}^{a} F_{a}^{\mu \rho} & =\frac{1}{4} g_{\nu}^{\rho} \tilde{F}_{\mu \sigma}^{a} F_{a}^{\mu \sigma} .
\end{aligned}
$$

It follows from the the symmetry properties of the space-time Riemann tensor that

$$
r_{\rho \sigma \mu \nu} F_{a}^{\nu \sigma} F^{a \mu \rho}=\frac{1}{2} r_{\mu \nu}^{\rho \sigma} F_{a}^{\mu \nu} F_{\rho \sigma}^{a},
$$

and, using (A.12) with $M_{1}=F, M_{2}=\tilde{F}, \widetilde{M}_{2}=-F$,

$$
\begin{aligned}
r_{\rho \sigma \mu \nu} \tilde{F}_{a}^{\nu \sigma} \tilde{F}^{a \mu \rho} & =\frac{1}{2} r_{\mu \nu}{ }^{\rho \sigma} \tilde{F}_{a}^{\mu \nu} \tilde{F}_{\rho \sigma}^{a} \\
& =2 r_{\nu}^{\mu} F_{\mu \rho}^{a} F_{a}^{\nu \rho}-\frac{1}{2} r F_{\mu \rho}^{a} F_{a}^{\mu \rho}-\frac{1}{2} r_{\mu \nu}{ }^{\rho \sigma} F_{a}^{\mu \nu} F_{\rho \sigma}^{a} .
\end{aligned}
$$

In addition:

$$
\begin{aligned}
F_{\mu \nu}^{a}\left[\mathcal{D}^{\mu}, \mathcal{D}_{\rho}\right] F_{a}^{\rho \nu}= & c_{a b c} F_{\mu \nu}^{a} F^{b \mu \rho} F^{c \nu} \\
& +r_{\nu}^{\mu} F_{\mu \rho}^{a} F_{a}^{\nu \rho}-\frac{1}{2} r_{\mu \nu}{ }^{\rho \sigma} F_{a}^{\mu \nu} F_{\rho \sigma}^{a} .
\end{aligned}
$$


It is convenient to isolate terms that do not contribute to the S-matrix, using the classical equations of motion:

$$
\begin{aligned}
& g^{-\frac{1}{2}} \mathcal{L}_{I}=-K_{I J} D_{\mu} \mathcal{D}^{\mu} z^{J}-\hat{V}_{I}-\frac{1}{x} \mathcal{D}_{a}\left(T^{a} z\right)^{J} K_{I J}-\frac{1}{2} f_{I}\left\{\begin{array}{l}
\mathcal{W} \\
\overline{\mathcal{W}}
\end{array}, \quad I, J=\left\{\begin{array}{l}
i, \bar{\jmath} \\
\bar{\imath}, j
\end{array},\right.\right. \\
& (x g)^{-\frac{1}{2}} \mathcal{L}_{a \mu}=(x g)^{-\frac{1}{2}} g_{\mu \nu} \frac{\partial \mathcal{L}}{\partial A_{\nu}^{a}}=\mathcal{D}^{\prime \prime \nu} \mathcal{F}_{a \nu \mu}+\tilde{\mathcal{F}}_{a \nu \mu} \frac{\partial^{\nu} y}{x} \\
& +\frac{i}{\sqrt{x}} K_{i \bar{m}}\left(\mathcal{D}_{\mu} \bar{z}^{\bar{m}}\left(T_{a} z\right)^{i}-\mathcal{D}_{\mu} z^{i}\left(T_{a} \bar{z}\right)^{\bar{m}}\right)
\end{aligned}
$$

The first of these gives, in particular $\left(M_{\psi}^{2}=m_{\psi} \bar{m}_{\psi}, M_{\lambda}^{2}=m_{\lambda} \bar{m}_{\lambda}\right)$ :

$$
\begin{aligned}
\frac{f_{i}}{\sqrt{g}} \mathcal{L}^{i}=\left(\frac{\bar{f}^{i}}{\sqrt{g}} \mathcal{L}_{i}\right)^{*}= & -\nabla^{2} x-i \nabla^{2} y-2 x^{4} \rho^{i} \rho_{i} \overline{\mathcal{W}}+\frac{1}{x}\left(\partial_{\nu} x+i \partial_{\nu} y\right)\left(\partial^{\nu} x+i \partial^{\nu} y\right) \\
& -2 x^{2} \rho_{i j} \mathcal{D}_{\mu} z^{j} \mathcal{D}^{\mu} z^{i}+2 x e^{-K}\left(2 \bar{a} A-\bar{a}^{i} A_{i}\right) \\
& +2 x\left(\hat{V}+M_{\psi}^{2}-M_{\lambda}^{2}\right) \\
-\frac{\partial_{\mu} x}{\sqrt{g} x} \mathcal{D}^{\mu} z^{i} \mathcal{L}_{i}+\text { h.c. }= & \left(\frac{\partial_{\mu} x}{x} \mathcal{D}^{\mu} z^{i} K_{i \bar{m}} D^{\nu} \mathcal{D}_{\nu} \bar{z}^{\bar{m}}+\text { h.c. }\right)-\frac{\nabla^{2} x}{x} V \\
& +\frac{\partial_{\mu} x \partial^{\mu} x}{x^{2}}\left(V+\frac{x}{4} F^{2}\right)+\frac{\partial_{\mu} y \partial^{\mu} x}{4 x} F \tilde{F}+\text { total derivative } \\
\frac{a+b x^{2} \rho^{i} \rho_{i}}{x \sqrt{g}} \mathcal{D}^{a}\left(T_{a} z\right)^{I} \mathcal{L}_{I}= & \frac{a+b x^{2} \rho^{i} \rho_{i}\left(2 K_{j \bar{m}} \mathcal{D}^{\mu} z^{i} \mathcal{D}_{\mu} \bar{z}^{\bar{m}} \mathcal{D}^{a} D_{i}\left(T_{a} z\right)^{j}+8 x \mathcal{D} M_{\psi}^{2}\right.}{x} \\
& -2 \mathcal{D}^{a} \mathcal{D}^{b} \mathcal{K}_{a b}-e^{-K}\left[\mathcal{D}^{a}\left(T_{a} z\right)^{i} A_{i j} \bar{A}^{j}+\text { h.c. }\right] \\
& +\left(K_{i \bar{n}} K_{j \bar{m}} \mathcal{D}^{\mu} z^{j}\left(T_{a} \bar{z}\right)^{\bar{m}}\left[\left(T_{a} z\right)^{i} \mathcal{D}_{\mu} \bar{z}^{\bar{n}}+\left(T_{a} \bar{z}\right)^{\bar{n}} \mathcal{D}_{\mu} z^{i}\right]\right. \\
& + \text { h.c. }) \\
& \left.\quad-\frac{\partial_{\mu} x}{x} \mathcal{D}^{a} K_{j \bar{m}}\left[\mathcal{D}^{\mu} z^{j}\left(T_{a} \bar{z}\right)^{\bar{m}}+\left(T_{a} z\right)^{j} \mathcal{D}^{\mu} \bar{z}^{\bar{m}}\right]\right) \\
+ & b x \mathcal{D}^{a}\left[\mathcal{D}_{\mu} z^{k} \rho^{j} \rho_{k j} K_{i \bar{m}}\left(\mathcal{D}_{\mu} \bar{z}^{\bar{m}}\left(T_{a} z\right)^{i}+\mathcal{D}_{\mu} z^{i}\left(T_{a} \bar{z}\right)^{\bar{m}}\right)+\text { h.c. }\right] \\
+ & \text { total derivative. }
\end{aligned}
$$

We absorb a part of the one loop correction into the Kähler potential; a shift $\delta K$ in the Kähler potential gives a shift $\Delta_{\delta K} \mathcal{L}$ in the Lagrangian:

$$
\begin{aligned}
\frac{1}{\sqrt{g}} \Delta_{\delta K} \mathcal{L}= & -\delta K \hat{V}+\delta K_{i \bar{m}}\left(e^{-K} \bar{A}^{i} A^{\bar{m}}+\mathcal{D}_{\mu} z^{i} \mathcal{D}^{\mu} \bar{z}^{\bar{m}}\right) \\
& -\left\{\delta K_{i}\left[e^{-K} \bar{A}^{i} A+\frac{1}{2 x} \mathcal{D}_{a}\left(T^{a} z\right)^{i}\right]+\text { h.c. }\right\}
\end{aligned}
$$


Taking $\delta K=\mathcal{D}$, the last equation in (A.18) can be written as

$$
\begin{aligned}
\frac{a+b x^{2} \rho^{i} \rho_{i}}{x \sqrt{g}} \mathcal{D}^{a}\left(T_{a} z\right)^{I} \mathcal{L}_{I}= & \left(a+b x^{2} \rho^{i} \rho_{i}\right)\left(\frac{2}{\sqrt{g}} \Delta_{\mathcal{D}} \mathcal{L}+2 \mathcal{D}\left[e^{-K} a \bar{a}-3 M_{\psi}^{2}-3 M_{\lambda}^{2}-\hat{V}\right]\right. \\
& +\left[K_{i \bar{n}} K_{j \bar{m}} \mathcal{D}_{\mu} z^{i} \mathcal{D}^{\mu} z^{j}\left(T_{a} \bar{z}\right)^{\bar{m}}\left(T_{a} \bar{z}\right)^{\bar{n}}+\text { h.c. }\right] \\
& +i \frac{\partial_{\mu} y}{x^{2}} \mathcal{D}^{a}\left[K_{i \bar{m}}\left(T_{a} z\right)^{i} \mathcal{D}_{\mu} \bar{z}^{\bar{m}}-\text { h.c. }\right] \\
& \left.-\frac{1}{x^{2}} \mathcal{D}\left[\partial_{\mu} x \partial^{\mu} x+\partial_{\mu} y \partial^{\mu} y\right]\right) \\
& +b x \mathcal{D}^{a}\left[\mathcal{D}_{\mu} z^{k} \rho^{j} \rho_{k j} K_{i \bar{m}}\left(\mathcal{D}_{\mu} \bar{z}^{\bar{m}}\left(T_{a} z\right)^{i}+\mathcal{D}_{\mu} z^{i}\left(T_{a} \bar{z}\right)^{\bar{m}}\right)+\text { h.c. }\right] \\
& + \text { total derivative. }
\end{aligned}
$$

\section{B. Matrix Elements and Supertraces}

In this Appendix we list matrix elements of operators appearing in Eqs. (4.72-4.75) and traces needed to evaluate the divergent contributions to the one-loop effective action (4.76). Notation and conventions are defined in Section 3.2, and the relevant part of the tree Lagrangian [42], [13] is ${ }^{8}$

$$
\begin{aligned}
\frac{1}{\sqrt{g}} \mathcal{L}(g, K, f)= & \frac{1}{2} r+K_{i \bar{m}} \mathcal{D}^{\mu} z^{i} \mathcal{D}_{\mu} \bar{z}^{\bar{m}}-\frac{x}{4} F_{\mu \nu} F^{\mu \nu}-\frac{y}{4} \tilde{F}_{\mu \nu} F^{\mu \nu}-V \\
& +\frac{i x}{2} \bar{\lambda} \not D \lambda+i K_{i \bar{m}}\left(\bar{\chi}_{L}^{\bar{m}} \not D \chi_{L}^{i}+\bar{\chi}_{R}^{i} \not D \chi_{R}^{\bar{m}}\right) \\
& +e^{-K / 2}\left(\frac{1}{4} f_{i} \bar{A}^{i} \bar{\lambda}_{R} \lambda_{L}-A_{i j} \bar{\chi}_{R}^{i} \chi_{L}^{j}+\text { h.c. }\right) \\
& +\left(i \bar{\lambda}_{R}^{a}\left[2 K_{i \bar{m}}\left(T_{a} \bar{z}\right)^{\bar{m}}-\frac{1}{2 x} f_{i} \mathcal{D}_{a}-\frac{1}{4} \sigma_{\mu \nu} F_{a}^{\mu \nu} f_{i}\right] \chi_{L}^{i}+\text { h.c. }\right) \\
& +\mathcal{L}_{\psi}+\text { four }- \text { fermion terms, } \\
\frac{1}{\sqrt{g}} \mathcal{L}_{\psi}= & \frac{1}{4} \bar{\psi}_{\mu} \gamma^{\nu}(i \not D+M) \gamma^{\mu} \psi_{\nu}-\frac{1}{4} \bar{\psi}_{\mu} \gamma^{\mu}(i \not D+M) \gamma^{\nu} \psi_{\nu}-\left[\frac{x}{8} \bar{\psi}_{\mu} \sigma^{\nu \rho} \gamma^{\mu} \lambda_{a} F_{\nu \rho}^{a}\right. \\
& \left.+\bar{\psi}_{\mu} \not \supset \bar{z}^{\bar{m}} K_{i \bar{m}} \gamma^{\mu} L \chi^{i}-\frac{1}{4} \bar{\psi}_{\mu} \gamma^{\mu} \gamma_{5} \lambda^{a} \mathcal{D}_{a}+i \bar{\psi}_{\mu} \gamma^{\mu} L \chi^{i} m_{i}+\text { h.c. }\right], \\
\bar{M}= & (M)^{\dagger}=e^{K / 2}(W R+\bar{W} L), \quad m_{i}=e^{-K / 2} A_{i} .
\end{aligned}
$$

If we define

$$
\mathrm{STr} F=\operatorname{Tr} F_{\Phi}-\frac{1}{4} \operatorname{Tr} F_{\Theta}-2 \operatorname{Tr} F_{g h}+2 \operatorname{Tr} F_{G h}, \quad-\frac{i}{2} T_{-}=\sqrt{g} \frac{\ln \Lambda^{2}}{32 \pi^{2}} T
$$

\footnotetext{
${ }^{8}$ In $I$ we defined $\epsilon^{0123}=1$; here we denote by $\epsilon^{\mu \nu \rho \sigma}$ the covariantly constant tensor. With this definition there is no factor $g^{-\frac{1}{2}}$ multiplying the $F \tilde{F}$ term in the Lagrangian.
} 
where $\operatorname{Tr} F_{\Theta}$ is defined below [see (B.24)], the effective Lagrangian (4.2) is

$$
\frac{1}{\sqrt{g}} \mathcal{L}_{1}=-\frac{\Lambda^{2}}{32 \pi^{2}} \mathrm{~S} \operatorname{Tr} H+\frac{\ln \Lambda^{2}}{32 \pi^{2}}\left[\mathrm{~S} \operatorname{Tr}\left(\frac{1}{2} H^{2}-\frac{1}{6} r H+\frac{1}{12} \hat{G}_{\mu \nu} \hat{G}^{\mu \nu}\right)+T\right]
$$

In the following subsections we list the matrix elements that were not included in I; the subscript 0 refers to the contributions without the Yang-Mills sector that are given in Appendix B of $\mathrm{I}$, except that ordinary derivatives are replaced by gauge covariant derivatives.

The contributions to $S \operatorname{Tr} H$ from each supermultiplet have been given in [45]; below we list the analogous contributions to $\mathrm{STr} H^{2}$ and $\mathrm{STr} G^{2}$; we drop all total derivatives.

\section{Boson matrix elements}

As in [41] we rescale the quantum gauge fields: $A_{\mu}=\sqrt{x} \mathcal{A}_{\mu}$. Then the operator $H_{\Phi}$ can be expressed as

$$
\begin{aligned}
Z_{\Phi} H_{\Phi}= & H+X+Y-N-S-K \\
\Phi^{T} Z_{\Phi} H_{\Phi} \Phi= & z^{I} H_{I J} z^{J}+h^{\mu \nu} X_{\mu \nu, \rho \sigma} h^{\rho \sigma}+2 h^{\mu \nu} Y_{\mu \nu I} z^{I}-\hat{\mathcal{A}}^{\mu} N_{\mu \nu} \hat{\mathcal{A}}^{\nu} \\
& -2 \hat{\mathcal{A}}^{\mu} S_{\mu I} z^{I}-2 h^{\mu \nu} K_{\mu \nu, \rho} \hat{\mathcal{A}}^{\rho}
\end{aligned}
$$

with, in addition to the matrix elements of $Z_{\Phi}$ given in $I$,

$$
Z_{i, a \mu}=Z_{\mu \nu, a \rho}=0, \quad Z_{a \mu, b \nu}=-g_{\mu \nu} \delta_{a b}
$$

Using the results of [41] and Section 2 above, the elements of $H, X, Y$ are modified with respect to those given in (B.3) of I by

$$
\begin{gathered}
H_{I J}=\left(H_{0}\right)_{I J}+\mathcal{D}_{I J}+q_{I}^{a} q_{a J}+v_{I J}-\left(V_{\mu} V^{\mu}\right)_{I J}, \quad q_{a}^{i}=-\frac{i}{\sqrt{x}}\left(T_{a} z\right)^{i}, \\
q_{i}^{a}=\frac{i}{\sqrt{x}}\left(T^{a} \bar{z}\right)^{\bar{m}} K_{i \bar{m}}, \quad v_{i \bar{m}}=v_{\bar{m} i}=\left(V_{\mu} V^{\mu}\right)_{i \bar{m}}=\left(V_{\mu} V^{\mu}\right)_{\bar{m} i}=0 \\
\left(V_{\mu} V^{\mu}\right)_{I J}=\frac{f_{I} f_{J}}{x f_{I J}} v_{I J}=\frac{1}{8 x^{2}} f_{I} f_{J}\left(\mathcal{F}_{a}^{\mu \nu} \mathcal{F}_{\mu \nu}^{a} \mp i \tilde{\mathcal{F}}_{a}^{\mu \nu} \mathcal{F}_{\mu \nu}^{a}\right), \\
v_{I J}-\left(V_{\mu} V^{\mu}\right)_{I J}=-\frac{x}{4} \rho_{I J}\left(\mathcal{F}_{a}^{\mu \nu} \mathcal{F}_{\mu \nu}^{a} \mp i \tilde{\mathcal{F}}_{a}^{\mu \nu} \mathcal{F}_{\mu \nu}^{a}\right), \quad I, J=\left\{\begin{array}{l}
i, j \\
\bar{\imath}, \bar{\jmath}
\end{array},\right. \\
Y_{\mu \nu I}=-\frac{1}{2}\left(D_{\mu} \mathcal{D}_{\nu}+D_{\nu} \mathcal{D}_{\mu}\right) K_{I J} z^{J}-\frac{1}{8} f_{I} F_{\mu \rho}^{a} F_{a \nu}{ }^{\rho} \pm \frac{i}{32} g_{\mu \nu} f_{I} F_{a}^{\sigma \rho} \tilde{F}_{\sigma \rho}^{a}, \quad I, J=\left\{\begin{array}{l}
i, \bar{j} \\
\bar{i}, j
\end{array}\right.
\end{gathered}
$$




$$
\begin{aligned}
X_{\mu \nu, \rho \sigma}= & \left(X_{0}\right)_{\mu \nu, \rho \sigma}-2 P_{\mu \nu, \rho \sigma} \mathcal{D}+\frac{1}{2} P_{\mu \nu, \rho \sigma} \mathcal{F}_{\lambda \tau}^{a} \mathcal{F}_{a}^{\lambda \tau}+\frac{1}{4}\left(\mathcal{F}_{\mu \rho}^{a} \mathcal{F}_{a \nu \sigma}+\mathcal{F}_{\nu \rho}^{a} \mathcal{F}_{a \mu \sigma}\right) \\
& -\frac{1}{16}\left(\mathcal{F}_{\mu \lambda}^{a} \mathcal{F}_{a \rho}{ }^{\lambda} g_{\nu \sigma}+\mathcal{F}_{\nu \lambda}^{a} \mathcal{F}_{a \rho}{ }^{\lambda} g_{\mu \sigma}+\mathcal{F}_{\mu \lambda}^{a} \mathcal{F}_{a \sigma}{ }^{\lambda} g_{\nu \rho}+\mathcal{F}_{\nu \lambda}^{a} \mathcal{F}_{a \sigma}{ }^{\lambda} g_{\mu \rho}\right)
\end{aligned}
$$

where $f_{I} \equiv f_{i}\left(\bar{f}_{\bar{\imath}}\right)$ for $I=i(\bar{\imath})$, etc.. The potential $V=\hat{V}+\mathcal{D}$ now includes the D-term $\mathcal{D}$ defined in (A.2) above:

$$
\begin{aligned}
\mathcal{D}_{i}= & -\frac{1}{2 x} f_{i} \mathcal{D}+\frac{1}{x} \mathcal{D}_{a} K_{i \bar{m}}\left(T^{a} \bar{z}\right)^{\bar{m}} \\
\mathcal{D}_{i}^{j}= & \frac{1}{2 x^{2}} f_{i} \bar{f}^{j} \mathcal{D}-\frac{1}{2 x^{2}} f_{i} \mathcal{D}_{a}\left(T^{a} z\right)^{j}-\frac{1}{2 x^{2}} \bar{f}^{j} \mathcal{D}_{a} K_{i \bar{n}}\left(T^{a} \bar{z}\right)^{\bar{n}} \\
& +\frac{1}{x}\left(T_{a} z\right)^{j} K_{i \bar{n}}\left(T^{a} \bar{z}\right)^{\bar{n}}+\frac{1}{x} \mathcal{D}_{a} D_{i}\left(T^{a} z\right)^{j} \\
\mathcal{D}_{i j}= & x \rho_{i j} \mathcal{D}-\frac{1}{2 x^{2}} \mathcal{D}_{a}\left(f_{i} K_{j \bar{m}}+f_{j} K_{i \bar{m}}\right)\left(T^{a} \bar{z}\right)^{\bar{m}} \\
& +\frac{1}{x} K_{j \bar{m}}\left(T^{a} \bar{z}\right)^{\bar{m}} K_{i \bar{n}}\left(T_{a} \bar{z}\right)^{\bar{n}}
\end{aligned}
$$

The additional nonvanishing elements of $Z_{\Phi} H_{\Phi}$ : are $-N_{a \mu, b \nu}, S_{a \mu, I}$, and $K_{\mu \nu, a \rho}$, with ${ }^{9}$

$$
\begin{aligned}
N_{a \mu, b \nu}= & g_{\mu \nu}\left(\mathcal{K}_{a b}+\mathcal{K}_{b a}-\frac{1}{2} \mathcal{F}_{a \rho \sigma} \mathcal{F}_{b}^{\rho \sigma}\right)+2 c_{a b c} F_{\mu \nu}^{c}+\frac{1}{2}\left(5 \mathcal{F}_{a \mu \rho} \mathcal{F}_{b \nu}{ }^{\rho}-\mathcal{F}_{a \nu \rho} \mathcal{F}_{b \mu}{ }^{\rho}\right) \\
& -\frac{x^{2}}{2} \rho_{i} \rho^{i}\left(\mathcal{F}_{a \mu \rho} \mathcal{F}_{b \nu}{ }^{\rho}+\mathcal{F}_{a \nu \rho} \mathcal{F}_{b \mu}{ }^{\rho}-\frac{1}{2} g_{\mu \nu} \mathcal{F}_{a \rho \sigma} \mathcal{F}_{b}^{\rho \sigma}\right) \\
& -g_{\mu \nu} \delta_{a b}\left(\frac{\nabla^{2} x}{2 x}-\frac{\partial_{\rho} x \partial^{\rho} x}{4 x^{2}}+\frac{\partial_{\rho} y \partial^{\rho} y}{2 x^{2}}\right) \\
& +\delta_{a b}\left(\frac{\nabla_{\mu} \partial_{\nu} x}{x}-\frac{\partial_{\mu} x \partial_{\nu} x}{x^{2}}+\frac{\partial_{\mu} y \partial_{\nu} y}{2 x^{2}}+r_{\mu \nu}\right) \\
S_{a \mu, I}= & \pm i \frac{2}{\sqrt{x}} K_{I K}\left[D_{\mu}\left(T_{a} z\right)^{K}-\frac{\partial_{\mu} x}{2 x}\left(T_{a} z\right)^{K}\right]-\frac{x}{2} \rho_{I J}\left(\mathcal{F}_{a \nu \mu} \mp i \tilde{\mathcal{F}}_{a \nu \mu}\right) \mathcal{D}^{\nu} z^{J} \\
& +\frac{1}{4 x} f_{I}\left[\mathcal{D}^{\prime \nu} \mathcal{F}_{a \nu \mu}+\frac{3 \partial^{\nu} x}{2 x}\left(\mathcal{F}_{a \nu \mu} \mp i \tilde{\mathcal{F}}_{a \nu \mu}\right)\right] \\
& +2 \mathcal{D}^{\nu} z^{K} K_{I K} \mathcal{F}_{a \mu \nu}, \quad I, J, K=\left\{\begin{array}{l}
i, j, \bar{k} \\
\bar{\imath}, \bar{j}, k
\end{array}\right. \\
K_{\mu \nu, \rho}^{a}= & -\frac{1}{2}\left(\mathcal{D}_{\mu}^{\prime \prime} \mathcal{F}_{\nu \rho}^{a}+\mathcal{D}_{\nu}^{\prime \prime} \mathcal{F}_{\mu \rho}^{a}\right)+\frac{1}{4}\left(g_{\mu \rho} \mathcal{D}^{\prime \sigma} \mathcal{F}_{\sigma \nu}^{a}+g_{\nu \rho} \mathcal{D}^{\prime \prime \sigma} \mathcal{F}_{\sigma \mu}^{a}\right) \\
& -\frac{\partial^{\sigma} x}{8 x}\left(g_{\mu \rho} \mathcal{F}_{\sigma \nu}^{a}+g_{\nu \rho} \mathcal{F}_{\sigma \mu}^{a}\right)+\frac{3 \partial^{\sigma} y}{8 x}\left(g_{\mu \rho} \tilde{\mathcal{F}}_{\sigma \nu}^{a}+g_{\nu \rho} \tilde{\mathcal{F}}_{\sigma \mu}^{a}\right) \\
& -\frac{1}{8 x}\left(\partial_{\mu} y \tilde{\mathcal{F}}_{\nu \rho}^{a}+\partial_{\nu} y \tilde{\mathcal{F}}_{\mu \rho}^{a}\right)-g_{\mu \nu} \tilde{\mathcal{F}}_{\sigma \rho}^{a} \frac{\partial^{\sigma} y}{4 x} .
\end{aligned}
$$

\footnotetext{
${ }^{9}$ In [37], [41], there is an additional graviton-gauge mass term $Q_{\mu \nu, a \rho}$; this term drops out when the prescription (4.23) is adopted.
} 
In writing the above expressions we used the notation in (2.2-3) and the first identity in (A.12) with $M_{1}=\mathcal{F}_{a}, M_{2}=\tilde{\mathcal{F}}_{b}, \widetilde{M}_{2}=-\mathcal{F}_{b}$. The inverse metric $Z^{-1}$ must be included in evaluating the traces of these operators, which are defined such that

$$
\begin{aligned}
& \operatorname{Tr} H_{\Phi}=\operatorname{Tr} H+\operatorname{Tr} X+\operatorname{Tr} N \\
& \operatorname{Tr} H_{\Phi}^{2}=\operatorname{Tr} H^{2}+\operatorname{Tr} X^{2}+\operatorname{Tr} N^{2}+2 \operatorname{Tr} Y^{2}-2 \operatorname{Tr} K^{2}-2 \operatorname{Tr} S^{2} .
\end{aligned}
$$

In the expressions for the traces given in Appendix C of II (the chiral-gauge sector is given below) space-time indices are raised with $g^{\mu \nu}$ and scalar indices are raised with $K^{i \bar{m}}$.

Finally we need

$$
\begin{aligned}
& \hat{G}_{\mu \nu}=\left(G_{z}+G_{G}+G_{g}+G_{g z}+G_{G z}+G_{g G}\right)_{\mu \nu}, \\
& \left(G_{\mu \nu}^{z}\right)_{J}^{I}=\left(G_{0 \mu \nu}^{z}\right)_{J}^{I} \pm i F_{\mu \nu}^{a} D_{J}\left(T_{a} z\right)^{I}, \quad I, J=\left\{\begin{array}{l}
i, j \\
\bar{\imath}, \bar{j}
\end{array}\right. \\
& \left(G_{\mu \nu}^{z}\right)_{J}^{I}=\left(G_{0 \mu \nu}^{z}\right)_{J}^{I}, \quad I, J=\left\{\begin{array}{l}
i, \bar{\jmath} \\
\bar{\imath}, j
\end{array},\right. \\
& \left(G_{\mu \nu}^{G}\right)_{\alpha \beta, \gamma \delta}=\left(G_{0 \mu \nu}^{G}\right)_{\alpha \beta, \gamma \delta}+\frac{1}{4}\left[\mathcal{F}_{\alpha \mu}^{a} \mathcal{F}_{a \gamma \nu} g_{\beta \delta}\right. \\
& \left.+\mathcal{F}_{\alpha \mu}^{a} \mathcal{F}_{a \delta \nu} g_{\beta \gamma}+\mathcal{F}_{\beta \mu}^{a} \mathcal{F}_{a \gamma \nu} g_{\alpha \delta}+\mathcal{F}_{\beta \mu}^{a} \mathcal{F}_{a \delta \nu} g_{\alpha \gamma}-(\mu \leftrightarrow \nu)\right], \\
& \left(G_{\mu \nu}^{g}\right)_{a \rho, b \sigma}=g_{\rho \sigma}\left(c_{a b c} F_{\mu \nu}^{c}+\frac{1}{2}\left[\mathcal{F}_{a \lambda \mu} \mathcal{F}_{b \nu}^{\lambda}-\mathcal{F}_{a \lambda \nu} \mathcal{F}_{b \mu}^{\lambda}\right]\right)+\delta_{a b} r_{\sigma \rho \mu \nu} \\
& -\delta_{a b}\left(\epsilon_{\rho \nu \sigma \lambda}\left[\frac{\nabla_{\mu} \partial^{\lambda} y}{2 x}-\frac{\partial^{\lambda} y \partial_{\mu} x}{2 x^{2}}\right]-(\mu \leftrightarrow \nu)\right) \\
& -\delta_{a b} \frac{1}{4 x^{2}}\left(\partial_{\lambda} y \partial^{\lambda} y g_{\rho \nu} g_{\mu \sigma}+\partial_{\sigma} y \partial_{\nu} y g_{\rho \mu}+\partial_{\rho} y \partial_{\mu} y g_{\nu \sigma}-(\mu \leftrightarrow \nu)\right) \\
& +\frac{1}{2}\left[\mathcal{F}_{a \sigma \mu} \mathcal{F}_{b \rho \nu}-\mathcal{F}_{a \rho \mu} \mathcal{F}_{b \sigma \nu}+x^{2} \rho_{i} \rho^{i}\left(\mathcal{F}_{a \mu \rho} \mathcal{F}_{b \nu \sigma}+\tilde{\mathcal{F}}_{a \mu \rho} \tilde{\mathcal{F}}_{b \nu \sigma}\right)\right. \\
& -(\mu \leftrightarrow \nu)] \\
& \left(G_{\mu \nu}^{g z}\right)_{a \rho, I}=\left(G_{\mu \nu}^{g z}\right)_{I, a \rho}=-\mathcal{D}_{\mu}\left[\frac{x}{2} \rho_{I}\left(\mathcal{F}_{a \nu \rho} \mp i \tilde{\mathcal{F}}_{a \nu \rho}\right)\right] \\
& -\epsilon_{\rho \mu \sigma \lambda} \frac{\partial^{\lambda} y}{8 x^{2}} f_{I}\left(\mathcal{F}_{a \nu}{ }^{\sigma} \mp i \tilde{\mathcal{F}}_{a \nu}{ }^{\sigma}\right)-(\mu \leftrightarrow \nu), \quad I=\left\{\begin{array}{l}
i \\
\bar{\imath}
\end{array},\right. \\
& \left(G_{\mu \nu}^{G z}\right)_{\alpha \beta, I}=-4\left(G_{\mu \nu}^{G z}\right)_{I, \alpha \beta}= \pm \frac{i x \rho_{I}}{2}\left[\tilde{\mathcal{F}}_{\alpha \mu}^{a} \mathcal{F}_{a \beta \nu}+\tilde{\mathcal{F}}_{\beta \mu}^{a} \mathcal{F}_{a \alpha \nu}-(\mu \leftrightarrow \nu)\right], I=\left\{\begin{array}{l}
i \\
\bar{\imath}
\end{array},\right. \\
& \left(G_{\mu \nu}^{g G}\right)_{a \rho, \alpha \beta}=\frac{1}{4}\left[\left(g_{\beta \rho} \mathcal{D}_{\mu}-\frac{\partial^{\lambda} y}{2 x} \epsilon_{\rho \mu \beta \lambda}\right) \mathcal{F}_{a \alpha \nu}\right.
\end{aligned}
$$




$$
\begin{aligned}
+ & \left.\left(g_{\alpha \rho} \mathcal{D}_{\mu}-\frac{\partial^{\lambda} y}{2 x} \epsilon_{\rho \mu \alpha \lambda}\right) \mathcal{F}_{a \beta \nu}-(\mu \leftrightarrow \nu)\right] \\
\left(G_{\mu \nu}^{g G}\right)_{\alpha \beta, a \rho}= & \left(g_{\beta \rho} \mathcal{D}_{\mu}-\frac{\partial^{\lambda} y}{2 x} \epsilon_{\rho \mu \beta \lambda}\right) \mathcal{F}_{a \alpha \nu}+\left(g_{\alpha \rho} \mathcal{D}_{\mu}-\frac{\partial^{\lambda} y}{2 x} \epsilon_{\rho \mu \alpha \lambda}\right) \mathcal{F}_{a \beta \nu} \\
& -g_{\alpha \beta}\left(\mathcal{D}_{\mu} \mathcal{F}_{a \rho \nu}-\frac{\partial^{\lambda} y}{2 x} \epsilon_{\rho \mu \sigma \lambda} \mathcal{F}_{a \nu}^{\sigma}\right)-(\mu \leftrightarrow \nu)
\end{aligned}
$$

\section{Fermion matrix elements}

As described in Chapters 3 and 4 , we take the Landau gauge condition $G=0$, where

$$
\begin{aligned}
G= & -\gamma^{\nu}(i \not D-\bar{M}) \psi_{\nu}-2\left(\not \supset z^{i} K_{i \bar{m}} R \chi^{\bar{m}}+\not \supset \bar{z}^{\bar{m}} K_{i \bar{m}} L \chi^{i}\right) \\
& +\frac{x}{2} \sigma^{\nu \rho} \lambda_{a} F_{\nu \rho}^{a}+2 i m_{I} \chi^{I}-\gamma_{5} \mathcal{D}_{a} \lambda^{a}
\end{aligned}
$$

which we implement by introducing an auxiliary field $\alpha$. After an appropriate shift in the gravitino field $\psi_{\mu}$, we obtain for the bilinear fermion couplings of the gravity sector:

$$
\begin{aligned}
\frac{1}{\sqrt{g}} \mathcal{L}_{\psi+\alpha}= & -\frac{1}{2} \bar{\psi}^{\mu}(i \not D-\bar{M}) \psi_{\mu}-\bar{\alpha}(i \not D+2 M) \alpha \\
& +i x \bar{\psi}_{\mu} F_{a}^{\mu} \lambda^{a}-2 \bar{\psi}_{\mu}\left(\mathcal{D}^{\mu} \bar{z}^{\bar{m}} K_{i \bar{m}} L \chi^{i}+\mathcal{D}^{\mu} z^{i} K_{i \bar{m}} R \chi^{\bar{m}}\right) \\
& -\bar{\alpha}\left(\frac{x}{2} \sigma^{\nu \rho} \lambda_{a} F_{\nu \rho}^{a}-2 i m_{I} \chi^{I}+\gamma_{5} \mathcal{D}_{a} \lambda^{a}\right) .
\end{aligned}
$$

To obtain the ghostino determinant we use the supersymmetry transformations [42]

$$
\begin{aligned}
i \delta \chi^{i} & =\frac{1}{2}\left(\not \mathcal{D} z^{i} R-i \bar{m}^{i} L\right) \epsilon, \quad i \delta \chi^{\bar{m}}=\left[\frac{1}{2}\left(\not \mathcal{D} \bar{z}^{\bar{m}} L-i m^{\bar{m}} R\right)\right] \epsilon \\
i \delta \psi_{\mu} & =\left(i D_{\mu}-\frac{1}{2} \gamma_{\mu} M\right) \epsilon, \quad i \delta \lambda^{a}=\left[\frac{i}{4} \gamma^{\mu} \gamma^{\nu} F_{\mu \nu}^{a}-\frac{1}{2 x} \gamma_{5} \mathcal{D}^{a}\right] \epsilon
\end{aligned}
$$

yielding

$$
\begin{aligned}
D^{2}+H_{G h}=\frac{\partial \delta G}{\partial \epsilon}= & D^{\mu} D_{\mu}-\frac{1}{2} \gamma^{\mu} \gamma^{\nu}\left[D_{\mu}, D_{\nu}\right]-i[\not D, M]-2 M \bar{M}+\bar{m}^{i} m_{i}+\mathcal{D} \\
& +2 i \bar{m}_{\bar{m}} \not \bar{z}^{\bar{m}} L+2 i m_{i} \not \supset z^{i} R+\frac{x}{2} \sigma_{\sigma \rho} F_{a}^{\sigma \rho}\left[\frac{1}{4} \sigma^{\mu \nu} F_{\mu \nu}^{a}-\frac{1}{x} \gamma_{5} \mathcal{D}^{a}\right] \\
& -\mathcal{D}_{\mu} z^{i} K_{i \bar{m}} \mathcal{D}^{\mu} \bar{z}^{\bar{m}}+\frac{1}{2} \gamma_{5}\left[\gamma^{\mu}, \gamma^{\nu}\right] \mathcal{D}_{\mu} \bar{z}^{\bar{m}} K_{i \bar{m}} \mathcal{D}_{\nu} z^{i}
\end{aligned}
$$

The metric for the gaugino field, as obtained from the classical supergravity Lagrangian given in (3.23), is $Z_{a b}=\delta_{a b} x$. Following [37] we rescale the gaugino field $\lambda=\sqrt{x} \lambda^{\prime}$, so for the rescaled field $\lambda^{\prime}, Z_{a b}=\delta_{a b}$. The matrix elements of $M_{\Theta}$ are given by (4.32), (3.25) and (B.9-10) of I and by

$$
M_{b}^{a}=\left(\bar{M}_{b}^{a}\right)^{*}=\delta_{b}^{a} m_{\lambda}, \quad m_{\lambda}=-\frac{e^{-K / 2}}{2 x} f_{k} \bar{A}^{k},
$$




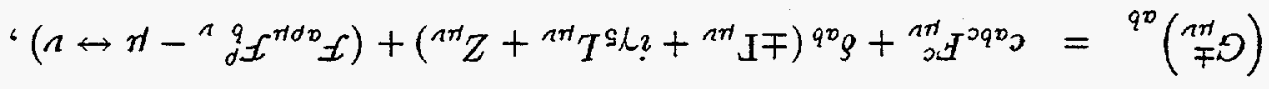

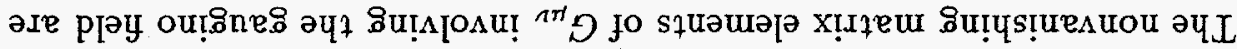

$(L I \cdot g)$

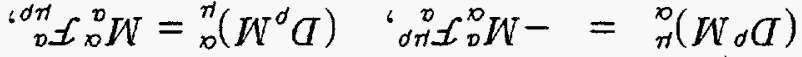

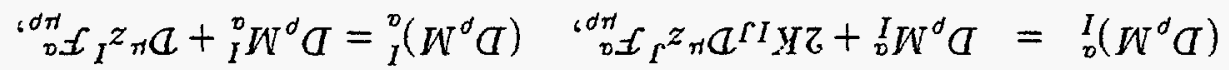

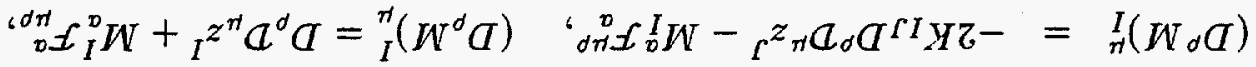

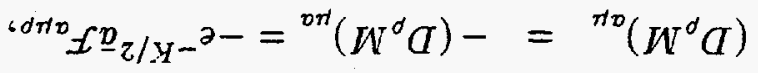

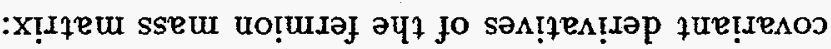

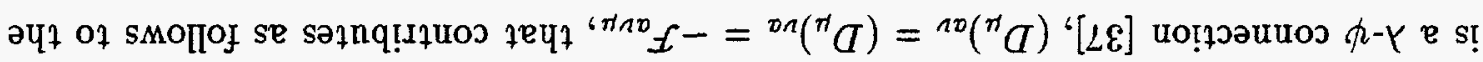

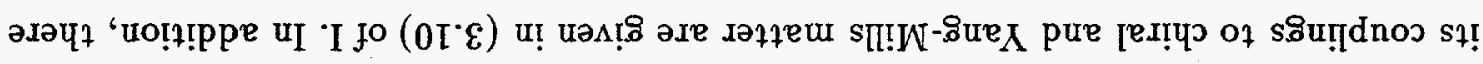

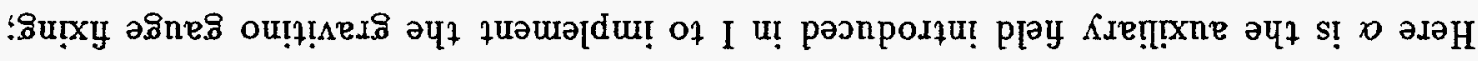
$\left(9 \mathrm{I}^{\cdot} \mathrm{g}\right)$

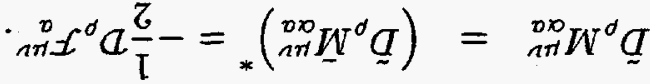

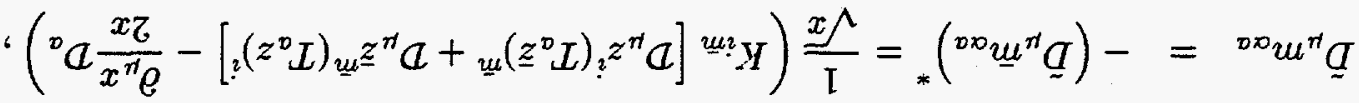

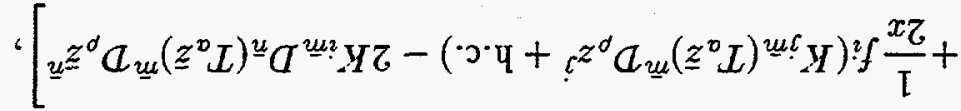

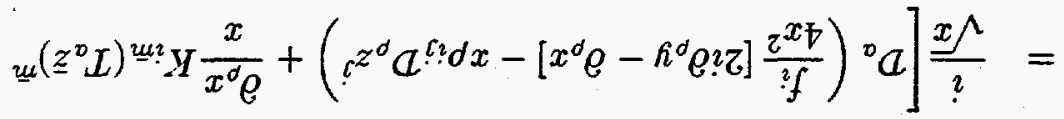

$$
\begin{aligned}
& { }_{*}\left({ }^{2} u u^{d} \underline{\underline{Q}}\right)={ }^{n} \cdot u^{d} \underline{\underline{G}}={ }^{20} u^{\delta} \underline{\underline{G}}
\end{aligned}
$$

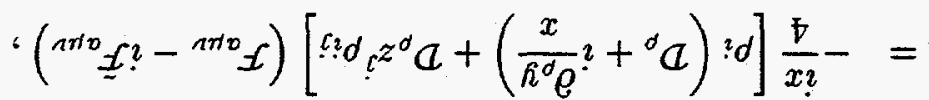

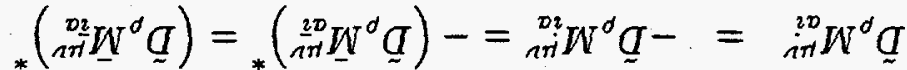

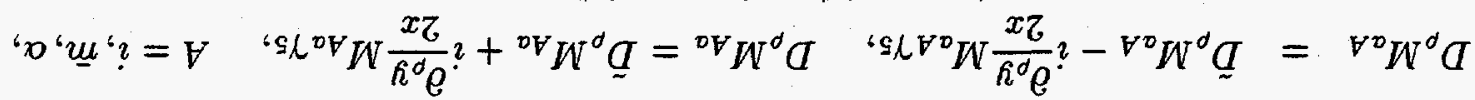

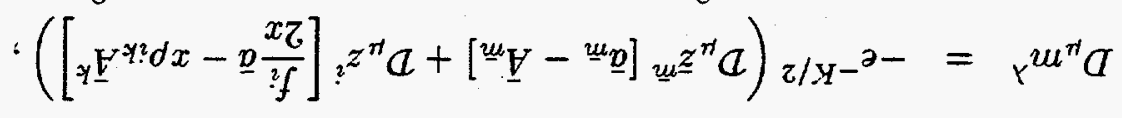

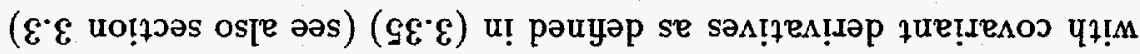

$(q \cdot g)$

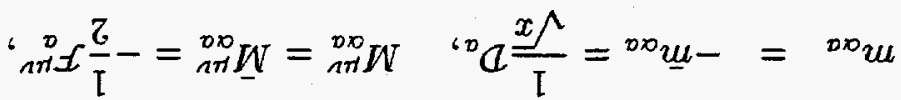

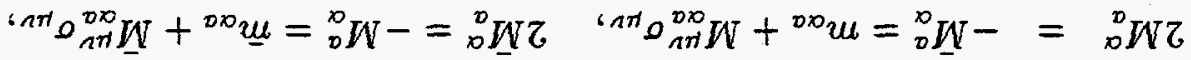

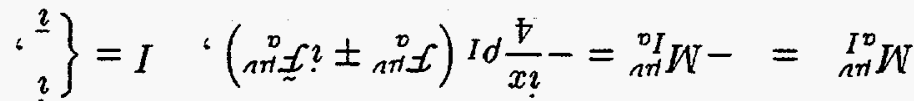

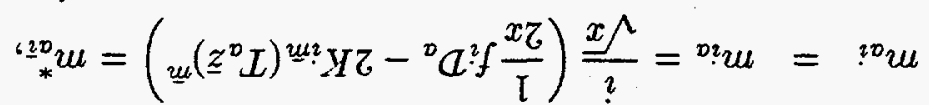

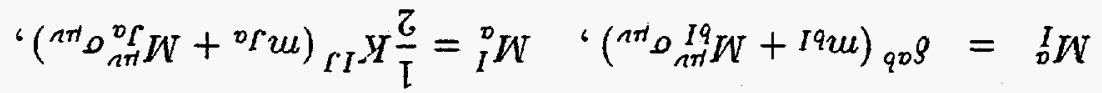




$$
\begin{aligned}
& \left(G_{\mu \nu}^{ \pm}\right)_{a \rho}=-\left[\left(\mathcal{D}_{\mu}+i \gamma_{5} L_{\mu}\right) \mathcal{F}_{a \rho \nu}-(\mu \leftrightarrow \nu)\right] \\
& \left(G_{\mu \nu}^{ \pm}\right)_{\rho a}=-\left[\left(\mathcal{D}_{\mu}-i \gamma_{5} L_{\mu}\right) \mathcal{F}_{a \rho \nu}-(\mu \leftrightarrow \nu)\right] .
\end{aligned}
$$

$\mathcal{D}_{\mu}$ is the gauge and general coordinate covariant derivative, $\Gamma_{\mu \nu}$ and $Z_{\mu \nu}$ are given in Section 3.3, and $\mathrm{d}^{10}$

$$
\mathcal{F}_{\mu \nu}=\sqrt{x} F_{\mu \nu}, \quad L_{\mu}=-\frac{\partial_{\mu} y}{2 x}, \quad L_{\mu \nu}=\frac{1}{2 x^{2}}\left(\partial_{\mu} x \partial_{\nu} y-\partial_{\nu} x \partial_{\mu} y\right)
$$

The other matrix elements of $G_{\mu \nu}$ are as given in Appendix (B.12) of I, except that now the chiral matter connection includes the gauge field:

$$
\left(G_{\mu \nu}\right)_{J}^{I}=\left(R_{\mu \nu}\right)_{J}^{I} \pm i F_{\mu \nu}^{a} D_{J}\left(T_{a} z\right)^{I}+\delta_{J}^{I}\left(Z_{\mu \nu} \pm \Gamma_{\mu \nu}\right), \quad I, J=\left\{\begin{array}{l}
i, j \\
\bar{\imath}, \bar{\jmath}
\end{array},\right.
$$

where $\left(R_{\mu \nu}\right)_{J}^{I}$ is defined in (B.8) of I, and the $\psi-\lambda$ connection gives an additional contribution to the gravitino matrix element of $G_{\mu \nu}$ :

$$
\left(G_{\mu \nu}^{ \pm}\right)_{\rho \sigma}=g_{\rho \sigma}\left( \pm \Gamma_{\mu \nu}+Z_{\mu \nu}\right)-r_{\rho \sigma \mu \nu}+\left(\mathcal{F}_{\rho \mu}^{a} \mathcal{F}_{a \sigma \nu}-\mu \leftrightarrow \nu\right)
$$

Finally, in the $8 \times 8$ matrix notation of (2.14-17), setting $G_{\mu \nu}=\tilde{G}_{\mu \nu}+i \gamma_{5} L_{\mu \nu}$,

$$
\begin{aligned}
H_{\Theta}= & M_{\Theta} M_{\Theta}+\frac{1}{4}\left[\gamma^{\mu}, \gamma^{\nu}\right] \tilde{G}_{\mu \nu}-i \not D M_{\Theta}-2 D^{\mu} M_{\mu \nu}^{\Theta} \gamma^{\nu}-4 \gamma^{\rho} \gamma_{\sigma} M_{\mu \rho}^{\Theta} M_{\Theta}^{\mu \sigma} \\
& -2 L_{\mu} L^{\mu}+i \tilde{D}^{\mu} L_{\mu} \gamma_{5}+2 i \gamma_{\mu} \gamma_{\rho} \gamma_{\nu} \gamma_{5}\left[L^{\rho}, M_{\Theta}^{\mu \nu}\right], \\
\hat{D}_{\mu}^{\Theta}= & \tilde{D}_{\mu}+2 \gamma^{\nu} M_{\mu \nu}^{\Theta}+\sigma_{\mu \nu} \gamma_{5} L^{\nu}, \\
\hat{G}_{\mu \nu}^{\Theta}= & \tilde{G}_{\mu \nu}+2 \gamma^{\rho}\left(\tilde{D}_{\mu} M_{\nu \rho}^{\Theta}-\tilde{D}_{\nu} M_{\mu \rho}^{\Theta}\right)+4 \gamma^{\rho} \gamma^{\sigma}\left(M_{\mu \rho}^{\Theta} M_{\nu \sigma}^{\Theta}-M_{\nu \rho}^{\Theta} M_{\mu \sigma}^{\Theta}\right) \\
& +\left[\sigma_{\rho \mu}\left(\gamma_{5} \tilde{D}_{\nu} L^{\rho}-2 i L_{\nu} L^{\rho}\right)-(\mu \leftrightarrow \nu)\right]-2 i L_{\rho} L^{\rho} \sigma_{\mu \nu}-4 i\left[\not L, M_{\mu \nu}^{\Theta}\right] \gamma_{5} \\
& -2\left[\gamma_{\mu}\left(\left\{L^{\rho}, \widetilde{M}_{\rho \nu}^{\Theta}\right\}-i\left[L^{\rho}, M_{\rho \nu}^{\Theta}\right] \gamma_{5}\right)+\left\{L_{\mu}, \widetilde{M}_{\nu \rho}^{\Theta}\right\} \gamma^{\rho}-(\mu \leftrightarrow \nu)\right], \\
M_{\Theta}= & m_{\Theta}+M_{\Theta}^{\mu \nu} \sigma_{\mu \nu}=m_{\Theta}+M_{\sigma} .
\end{aligned}
$$

Then, defining $H_{\Theta}=H_{1}+H_{2}+H_{3}$, with

$$
\begin{aligned}
H_{1} & =M_{\Theta} M_{\Theta}-4 \gamma^{\rho} \gamma_{\sigma} M_{\mu \rho}^{\Theta} M_{\Theta}^{\mu \sigma} \\
H_{2} & =-i \not D M_{\Theta}-2 \gamma^{\nu} D^{\mu} M_{\mu \nu}^{\Theta}+2 i \gamma_{\mu} \gamma_{\rho} \gamma_{\nu} \gamma_{5}\left[L^{\rho}, M_{\Theta}^{\mu \nu}\right] \\
H_{3} & =\frac{1}{4}\left[\gamma^{\mu}, \gamma^{\nu}\right] \tilde{G}_{\mu \nu}-2 L_{\mu} L^{\mu}+i \tilde{D}^{\mu} L_{\mu} \gamma_{5} \\
G_{\mu \nu}^{\prime} & =\tilde{G}_{\mu \nu}-Z_{\mu \nu},
\end{aligned}
$$

\footnotetext{
${ }^{10}$ We use the notation $L_{\mu}, L_{\mu \nu}$, to denote the field operators defined in (B.19), and also the matrices defined by these fields multiplying the unit projection operator in the space of gauginos, as in (4.41-43), (3.36-37), (B.22), etc.
} 
we find the following traces ( $\operatorname{Tr}$ includes the Dirac trace): $\operatorname{Tr} 1 \equiv 8 \operatorname{Tr} 1$, where $\operatorname{Tr}$ is over internal symmetry indices only):

$$
\begin{aligned}
\frac{1}{8} \operatorname{Tr} H_{1}= & \frac{1}{8} \operatorname{Tr}\left[m_{\Theta} m_{\Theta}-2 M_{\mu \nu}^{\Theta} M_{\Theta}^{\mu \nu}\right]=\operatorname{Tr}\left[\bar{m} m-2 \bar{M}_{\mu \nu} M^{\mu \nu}\right] \\
= & \frac{1}{8} \operatorname{Tr}\left(M_{\Theta}^{2}\right)_{0}+4 \mathcal{K}_{a}^{a}-2 \mathcal{D}\left(1-x^{2} \rho^{i} \rho_{i}\right)+N_{G} M_{\lambda}^{2}+\frac{x}{2} F_{\mu \nu}^{a} F_{a}^{\mu \nu} \\
\frac{1}{8} \operatorname{Tr} H_{1}^{2}= & \frac{1}{8} \operatorname{Tr}\left[\left(m_{\Theta} m_{\Theta}\right)^{2}+\left(\sigma_{\mu \nu} \sigma_{\rho \sigma} M_{\Theta}^{\mu \nu} M_{\Theta}^{\rho \sigma}\right)^{2}+4 m_{\Theta} M_{\Theta}^{\mu \nu} m_{\Theta} M_{\mu \nu}^{\ominus}\right. \\
& \left.+16 M_{\Theta}^{\mu \nu} M_{\rho \nu}^{\Theta}\left(M_{\mu \sigma}^{\Theta} M_{\Theta}^{\rho \sigma}-M_{\Theta}^{\rho \sigma} M_{\mu \sigma}^{\Theta}\right)\right] \\
= & \operatorname{Tr}\left[(\bar{m} m)^{2}+2 \bar{m} M^{\mu \nu} \bar{m}_{\mu \nu}+2 \bar{M}^{\mu \nu} m \bar{M}_{\mu \nu} m+4 \bar{M}_{\mu \nu} M_{\rho \sigma} \bar{M}^{\mu \nu} M^{\rho \sigma}\right. \\
& \left.+8\left(\bar{M}^{\mu \nu} M_{\mu \nu}\right)^{2}-16 \bar{M}^{\mu \nu} M^{\rho \sigma} \bar{M}_{\mu \rho} M_{\nu \sigma}\right]
\end{aligned}
$$

and using (A.12), partial integration and the relation

$$
\not D \gamma^{\mu} \gamma^{\nu} M_{\mu \nu}=2 \gamma^{\nu} D^{\mu} M_{\mu \nu}+2 i \gamma^{\nu} D^{\mu} \widetilde{M}_{\mu \nu} \gamma_{5}
$$

we obtain

$$
\begin{aligned}
-\frac{1}{8} \operatorname{Tr} H_{2}^{2}= & \left.-\frac{1}{8} \operatorname{Tr}\left\{-i \not D m_{\Theta}+2 \gamma_{\nu}\left[L_{\mu}, \widetilde{M}_{\Theta}^{\mu \nu}\right]+2 i \gamma_{\nu} \gamma_{5} \tilde{D}_{\mu} \widetilde{M}_{\Theta}^{\mu \nu}\right\}\right\}^{2} \\
= & \operatorname{Tr}\left\{\tilde{D}_{\mu} \bar{m} \tilde{D}^{\mu} m-4 \tilde{D}_{\mu} \widetilde{\bar{M}}^{\mu \nu} \tilde{D}^{\rho} \widetilde{M}_{\rho \nu}-4\left[L_{\mu} \widetilde{\bar{M}}^{\mu \rho}\right]\left[L^{\nu}, \widetilde{M}_{\nu \rho}\right]\right. \\
& \left.+\left[L_{\mu}, \bar{m}\right]\left[L^{\mu}, m\right]-i\left(\left[\hat{L}_{\mu \nu}, \bar{m}\right] \widetilde{M}^{\mu \nu}+\left[\hat{L}_{\mu \nu}, m\right] \widetilde{\bar{M}}^{\mu \nu}\right)\right\}
\end{aligned}
$$

where $\hat{L}_{\mu \nu}$ is defined in (4.43). The remaining traces needed to evaluate $\operatorname{Tr} H_{\Theta}, \operatorname{Tr} H_{\Theta}^{2}$ are:

$$
\begin{aligned}
\frac{1}{2} \operatorname{Tr} H_{3}= & \left(N+N_{G}+5\right) r-2 N_{G} \frac{\partial_{\mu} y \partial^{\mu} y}{x^{2}} \\
\frac{1}{2} \operatorname{Tr} H_{3}^{2}= & N_{G} \operatorname{Tr} h_{3}^{2}+(N+5) \frac{r^{2}}{4}-\operatorname{Tr}\left(\left[\Gamma_{\mu}^{\prime}, L^{\mu}\right]\right)^{2}-\frac{1}{4} \operatorname{Tr}\left(G_{\mu \nu}^{\prime} G^{\mu \nu}\right) \\
\frac{1}{2} \operatorname{Tr}\left(H_{1} H_{3}\right)= & \frac{1}{2} \operatorname{Tr}\left[\left(\frac{r}{4}-2 L_{\mu} L^{\mu}\right) H_{1}-2 M_{\mu \nu}^{\Theta} \widetilde{M}_{\Theta}^{\mu \nu} \tilde{D}_{\rho} L^{\rho}-i G_{\mu \nu}^{\prime}\left\{M_{\Theta}^{\mu \nu}, m_{\Theta}\right\}\right] \\
\frac{1}{2} \operatorname{Tr} \hat{G}_{\mu \nu}^{\Theta} \hat{G}_{\Theta}^{\mu \nu}= & \frac{1}{2} \operatorname{Tr}\left\{\tilde{G}_{\mu \nu} \tilde{G}^{\mu \nu}+16 \tilde{G}_{\mu \nu} M_{\Theta}^{\mu \rho} M_{\Theta}^{\nu \sigma} \gamma_{\rho} \gamma_{\sigma}\right. \\
& +8 \tilde{D}_{\mu} M_{\nu \rho}^{\ominus}\left(\tilde{D}^{\mu} M_{\Theta}^{\nu \rho}-\tilde{D}^{\nu} M_{\Theta}^{\mu \rho}\right)+4\left[\Gamma_{\mu}^{\prime}, L_{\nu}\right]\left[\Gamma^{\prime \mu}, L^{\nu}\right] \\
& +16\left(\tilde{D}_{\mu} M_{\Theta}^{\mu \nu}\left\{L^{\rho}, \widetilde{M}_{\rho \nu}^{\Theta}\right\}-\tilde{D}_{\mu} \widetilde{M}_{\Theta}^{\mu \nu}\left\{L^{\rho}, M_{\rho \nu}^{\Theta}\right\}\right) \\
& +2\left(\left[\Gamma_{\mu}^{\prime}, L^{\mu}\right]\right)^{2}-32\left(M_{\mu \nu}^{\Theta} M_{\Theta}^{\mu \nu} M_{\rho \sigma}^{\Theta} M_{\Theta}^{\rho \sigma}+M_{\nu \rho}^{\Theta} M_{\Theta}^{\mu \rho} M_{\mu \sigma}^{\Theta} M_{\Theta}^{\nu \sigma}\right)
\end{aligned}
$$




$$
\begin{aligned}
& -32 M_{\mu \sigma}^{\ominus} M_{\nu \rho}^{\ominus}\left(M_{\Theta}^{\mu \sigma} M_{\Theta}^{\nu \rho}-3 M_{\Theta}^{\nu \sigma} M_{\Theta}^{\mu \rho}\right)+80 L_{\mu} L^{\nu} M_{\Theta}^{\mu \rho} M_{\nu \rho}^{\ominus} \\
& \left.-80 L_{\rho} L^{\rho} M_{\Theta}^{\mu \nu} M_{\mu \nu}^{\ominus}+24 \tilde{D}_{\mu} L^{\mu} \widetilde{M}_{\nu \rho}^{\ominus} M_{\Theta}^{\nu \rho}\right\}+N_{G} \operatorname{Tr}\left(\hat{g}^{2}-\tilde{g}^{2}\right)
\end{aligned}
$$

where $\operatorname{Tr} h_{3}^{2}, \operatorname{Tr} \hat{g}^{2}, \operatorname{Tr} \tilde{g}^{2}$ are given in (C.66) of II, and $\Gamma_{\mu}^{\prime}$ is the gaugino-gravitino connection.

\section{Ghost matrix elements}

For the gravitino ghost, $H_{G h}$ is defined by (B.14). For the bosonic ghosts we have

$$
\begin{gathered}
H_{g h}^{\mu \nu}=\left(H_{g h}^{\mu \nu}\right)_{0}+\frac{3}{2} \mathcal{F}_{\mu \rho}^{a} \mathcal{F}_{a \nu}{ }^{\rho} \\
H_{g h}^{a b}=\mathcal{K}_{a b}+\mathcal{K}_{b a}-\frac{1}{2} \mathcal{F}_{\mu \nu}^{a} \mathcal{F}_{b}^{\mu \nu}-\delta_{a b}\left(\frac{\nabla^{2} x}{2 x}-\frac{\partial_{\mu} x \partial^{\mu} x}{4 x^{2}}\right) \\
H_{\mu a}^{g h}=\frac{1}{\sqrt{2}} \mathcal{D}^{\nu} \mathcal{F}_{a \mu \nu}+\mathcal{F}_{a \mu \nu} \frac{\partial^{\nu} x}{\sqrt{2} x}+\sqrt{2} q_{a I} \mathcal{D}_{\mu} z^{I} \\
\left(H^{g h}\right)^{a}{ }_{\nu}=-\frac{1}{\sqrt{2}} \mathcal{D}^{\mu} \mathcal{F}_{\nu \mu}^{a}-\mathcal{F}_{\nu \mu}^{a} \frac{\partial^{\mu} x}{\sqrt{2} x}-\sqrt{2} q_{I}^{a} \mathcal{D}_{\nu} z^{I} \\
\left(\hat{G}_{\mu \nu}^{g h}\right)_{\rho \sigma}=-r_{\rho \sigma \mu \nu}+\frac{1}{2}\left(\mathcal{F}_{\rho \mu}^{a} \mathcal{F}_{a \sigma \nu}-(\mu \leftrightarrow \nu)\right) \\
\left(\hat{G}_{\mu \nu}^{g h}\right)_{a b}=c_{a b c} F_{\mu \nu}^{c}+\frac{1}{2}\left(\mathcal{F}_{a \rho \mu} \mathcal{F}_{b \nu}^{\rho}-(a \leftrightarrow b)\right) \\
\left(\hat{G}_{\mu \nu}^{g h}\right)^{a}{ }_{\rho}=\left(\hat{G}_{\mu \nu}^{g h}\right)_{\rho}^{a}=-\frac{1}{\sqrt{2}}\left(\mathcal{D}_{\mu} \mathcal{F}_{\rho \nu}^{a}-\mathcal{D}_{\nu} \mathcal{F}_{\rho \mu}^{a}\right)
\end{gathered}
$$

\section{Mixed chiral-gauge supertraces}

The following illustrates the evaluation of the supertraces. We give an outline and the result of the evaluation of the traces which give rise to divergent contributions to the effective action [as derived in Section 4.3)] only for the mixed chiral-gauge sector. The reason other sectors are not included is brevity. All other contributions can be found in Appendix $\mathrm{C}$ of II. The results from different sectors are then added up into the full one-loop effective Lagrangian which was presented in Chapter 4.

For the bose sector we have $H_{\Phi}^{\chi g}=-S$, and

$$
\begin{aligned}
\operatorname{Tr} S^{2}= & \frac{8}{x} K_{i \bar{m}}\left[D_{\mu}\left(T_{a} z\right)^{i}\right]\left[D^{\mu}\left(T_{a} \bar{z}\right)^{\bar{m}}\right]-4 \frac{\partial^{\mu} x}{x^{2}}\left[\left(T_{a} z\right)^{i} K_{i \bar{m}} D_{\mu}\left(T^{a} \bar{z}\right)^{\bar{m}}+\text { h.c. }\right] \\
& +2 \mathcal{K}_{a}^{a} \frac{\partial_{\mu} x \partial^{\mu} x}{x^{2}}-x\left[\rho_{i j}\left(T^{a} z\right)^{i}\left(T_{b} z\right)^{j} F_{a}^{\nu \mu}\left(F_{\nu \mu}^{b}-i \tilde{F}_{\nu \mu}^{b}\right)+\text { h.c. }\right] \\
& -2 i\left[x \rho_{\bar{m} i j} \mathcal{D}_{\nu} \bar{z}^{\bar{m}}\left(T^{a} z\right)^{i} \mathcal{D}_{\mu} z^{j}\left(F_{a}^{\mu \nu}-i \tilde{F}_{a}^{\mu \nu}\right)-\text { h.c. }\right]
\end{aligned}
$$




$$
\begin{aligned}
& +\frac{x^{2}}{2} \rho_{i} \rho^{i}\left(\mathcal{D}_{\nu}^{\prime \prime} \mathcal{F}_{a}^{\nu \rho}+\frac{\partial_{\nu} y}{x} \tilde{\mathcal{F}}_{a}^{\nu \rho}\right)^{2}+x \rho_{i} \rho^{i} F_{a}^{\nu \mu} F_{\rho \mu}^{a}\left(\frac{\partial_{\nu} y \partial^{\rho} y}{2}+\frac{5 \partial_{\nu} x \partial^{\rho} x}{4}\right) \\
& -x \rho_{i} \rho^{i}\left[F_{a}^{\nu \mu} F_{\nu \mu}^{a}\left(\frac{9 \partial_{\rho} x \partial^{\rho} x}{16}+\frac{\partial_{\rho} y \partial^{\rho} y}{4}\right)+\tilde{F}_{a}^{\nu \mu} F_{\nu \mu}^{a} \frac{\partial_{\rho} y \partial^{\rho} x}{8}\right] \\
& -\frac{\sqrt{x}}{4}\left(\mathcal{D}_{\nu}^{\prime \prime} \mathcal{F}_{a}^{\nu \rho}+\frac{\partial_{\nu} y}{x} \tilde{\mathcal{F}}_{a}^{\nu \rho}\right)\left[\bar{f}^{i} \rho_{i j} \mathcal{D}^{\mu} z^{j}\left(F_{\mu \rho}^{a}-i \tilde{F}_{\mu \rho}^{a}\right)+\text { h.c. }\right] \\
& +\frac{\sqrt{x}}{2} x \rho^{i} \rho_{i}\left(\mathcal{D}_{\nu}^{\prime \prime} \mathcal{F}_{a}^{\nu \rho}+\frac{\partial_{\nu} y}{x} \tilde{\mathcal{F}}_{a}^{\nu \rho}\right)\left(\partial^{\mu} x F_{\mu \rho}^{a}-2 \partial^{\mu} y \tilde{F}_{\mu \rho}^{a}\right) \\
& -F_{a}^{\nu \mu} F_{\rho \mu}^{a}\left\{\left[\left(\frac{\partial_{\nu} x}{2}+i \frac{\partial_{\nu} y}{4}\right) \mathcal{D}^{\rho} z^{j} \rho_{i j} \bar{f}^{i}+\text { h.c. }\right]+x^{3} \mathcal{D}_{\nu} z^{i} \mathcal{D}^{\rho} \bar{z}^{\bar{m}} \rho_{i j} \rho_{\bar{m}}^{j}\right\} \\
& +F_{a}^{\nu \mu} F_{\nu \mu}^{a}\left\{\left[\left(\frac{3 \partial_{\rho} x}{16}+i \frac{\partial_{\rho} y}{8}\right) \mathcal{D}^{\rho} z^{j} \rho_{i j} \bar{f}^{i}+\text { h.c. }\right]+\frac{x^{3}}{4} \mathcal{D}_{\rho} z^{i} \mathcal{D}^{\rho} \bar{z}^{\bar{m}} \rho_{i j} \rho_{\tilde{m}}^{j}\right\} \\
& -\frac{1}{16} \tilde{F}_{a}^{\nu \mu} F_{\nu \mu}^{a}\left[i\left(\partial_{\rho} x+i \partial_{\rho} y\right) \mathcal{D}^{\rho} z^{j} \rho_{i j} \bar{f}^{i}+\text { h.c. }\right] \\
& +8 x F_{a}^{\nu \mu} F_{\nu \mu}^{b} \mathcal{K}_{b}^{a}+4 i\left(\frac{2}{\sqrt{x}} \mathcal{D}_{\mu}^{\prime \prime} \mathcal{F}^{\mu \nu}-\frac{\partial_{\mu} x}{x} F_{a}^{\mu \nu}\right)\left[\left(T^{a} z\right)^{i} K_{i \bar{m}} \mathcal{D}_{\nu} \bar{z}^{\bar{m}}-\text { h.c. }\right] \\
& -2 \frac{\partial^{\mu} x}{\sqrt{x}} F_{\mu \rho}^{a} \mathcal{D}_{\nu}^{\prime \prime} \mathcal{F}_{a}^{\nu \rho}-\frac{3 \partial_{\nu} y \partial^{\nu} x}{4 x} F_{a}^{\mu \rho} \tilde{F}_{\mu \rho}^{a}-F_{a}^{\nu \mu} F_{\rho \mu}^{a} \frac{\partial_{\nu} x \partial^{\rho} x}{x} \\
& +2 x F_{a}^{\nu \mu} F_{\rho \mu}^{a}\left[4 \mathcal{D}^{\rho} z^{i} \mathcal{D}_{\nu} \bar{z}^{\bar{m}} K_{i \bar{m}}+x\left(\mathcal{D}^{\rho} z^{i} \mathcal{D}^{\nu} z^{j} \rho_{i j}+\text { h.c. }\right)\right] \\
& -\frac{i}{2} x^{2} \tilde{F}_{a}^{\nu \mu} F_{\nu \mu}^{a}\left(\mathcal{D}_{\rho} z^{j} \mathcal{D}^{\rho} z^{i} \rho_{i j}-\text { h.c. }\right) .
\end{aligned}
$$

In writing this expression we dropped total derivatives and used (A.10) and (A.12-A.14), as well as the Yang-Mills Bianchi identity. In addition we used (A.3-5) and (A.8) and

$$
\begin{aligned}
& \sqrt{x} \rho_{i j}\left(\mathcal{F}_{a}^{\nu \mu}-i \tilde{\mathcal{F}}_{a}^{\nu \mu}\right) \mathcal{D}_{\nu} z^{i} D_{\mu}\left(T^{a} z\right)^{j}=-\mathcal{D}_{\nu} z^{i} \rho_{i j}\left(T^{a} z\right)^{j}\left(\sqrt{x} \mathcal{D}_{\mu}^{\prime \prime} \mathcal{F}_{a}^{\nu \mu}-i \tilde{F}_{a}^{\nu \mu} \partial_{\mu} x\right) \\
& -x\left(T^{a} z\right)^{j}\left(F_{a}^{\nu \mu}-i \tilde{F}_{a}^{\nu \mu}\right)\left(\rho_{i j} D_{\mu} \mathcal{D}_{\nu} z^{i}+\rho_{\bar{m} i j} \mathcal{D}_{\mu} \bar{z}^{\bar{m}} \mathcal{D}_{\nu} z^{i}\right)+\text { total derivative } \\
& -i F_{\mu \nu}^{a}\left[\mathcal{D}^{\mu} z^{i} \mathcal{D}^{\nu} \bar{z}^{\bar{m}} K_{j \bar{m}} D_{i}\left(T_{a} z\right)^{j}-\text { h.c. }\right]=\text { total deriv. } \\
& \quad+i \mathcal{D}^{\mu} F_{\mu \nu}^{a} K_{i \bar{m}}\left[\mathcal{D}^{\nu} \bar{z}^{\bar{m}}\left(T_{a} z\right)^{i}-\mathcal{D}^{\nu} z^{i}\left(T_{a} \bar{z}\right)^{\bar{m}}\right]+x F_{\mu \nu}^{a} F_{b}^{\mu \nu} \mathcal{K}_{a}^{b}, \\
& F_{\mu \nu}^{a} D^{\mu} \mathcal{D}^{\nu} z^{I}= \pm \frac{i}{2} F_{\mu \nu}^{a} F_{b}^{\mu \nu}\left(T^{b} z\right)^{I}, \quad I=\left\{\begin{array}{l}
i \\
\bar{\imath}
\end{array}\right.
\end{aligned}
$$

To evaluate the fermion matrix elements we use (4.46); we have

$$
\begin{aligned}
\frac{1}{8} \operatorname{Tr}\left(H_{1}^{\chi g}\right)^{2}= & \operatorname{Tr} h_{\chi g}^{2}+2\left[\left(\bar{m} M^{\mu \nu}\right)_{a}^{i}\left(\bar{m} M_{\mu \nu}\right)_{i}^{a}+\left(\bar{M}^{\mu \nu} m\right)_{a}^{i}\left(\bar{M}_{\mu \nu} m\right)_{i}^{a}+\text { h.c. }\right] \\
= & -T_{4}^{\chi g}+e^{-K} \mathcal{D}\left(2 a_{i} \bar{a}^{i}+8 a \bar{a}\right)+2 \mathcal{D}\left(\hat{V}-M_{\psi}^{2}\right) \\
& +\frac{e^{-K}}{x}\left[4\left(T_{a} z\right)^{i} A_{i j}\left(T^{a} z\right)^{j}(\bar{a}-\bar{A})-2\left(\left(T_{a} z\right)^{i} \mathcal{D}^{a} A_{i j} \bar{a}^{j}+\text { h.c. }\right)\right] \\
& +4 \frac{e^{-K}}{x}\left(T_{a} z\right)^{i}\left(T^{a} \bar{z}\right)^{\bar{n}} A_{k i} \bar{A}_{\bar{n}}^{k}+2 M_{\lambda}^{2}\left[2 \mathcal{K}_{a}^{a}+\left(3 x^{2} \rho^{i} \rho_{i}-4\right) \mathcal{D}\right] \\
& +2 \frac{e^{-K}}{x}\left\{a_{i}(\bar{a}-\bar{A})\left[\bar{f}^{i} \mathcal{D}-\left(T^{a} z\right)^{i} \mathcal{D}_{a}\right]+\text { h.c. }\right\},
\end{aligned}
$$




$$
\begin{aligned}
-\frac{1}{8} \operatorname{Tr}\left(H_{2}^{\alpha g}\right)^{2}= & 2\left(\tilde{D}_{\mu} \bar{m}\right)_{a}^{i}\left(\tilde{D}^{\mu} m\right)_{i}^{a}-8\left(\tilde{D}_{\mu} \bar{M}^{\mu \nu}\right)_{a}^{i}\left(\tilde{D}^{\rho} M_{\rho \nu}\right)_{i}^{a} \\
& +\left\{\left[\hat{L}_{\mu \nu}, \bar{m}\right]_{a}^{i}\left(M^{\mu \nu}\right)_{i}^{a}+\left[\hat{L}_{\mu \nu}, \bar{m}\right]_{\bar{m}}^{a}\left(M^{\mu \nu}\right)_{a}^{\bar{m}}+\text { h.c. }\right\} \\
& +2\left[L_{\mu}, \bar{m}\right]_{a}^{i}\left[L^{\mu}, m\right]_{i}^{a}+8 L^{\rho} L_{\mu}\left(\bar{M}_{\rho \nu}\right)_{a}^{i}\left(M^{\mu \nu}\right)_{i}^{a}
\end{aligned}
$$

with

$$
\begin{aligned}
& -8\left(\tilde{D}_{\mu} \bar{M}^{\mu \nu}\right)_{a}^{i}\left(\tilde{D}^{\rho} M_{\rho \nu}\right)_{i}^{a}=-\frac{x^{2} \rho_{i} \rho^{i}}{4}\left(\mathcal{D}_{\nu}^{\prime \prime} \mathcal{F}^{\nu \mu}+\frac{\partial_{\nu} y}{\sqrt{x}} \tilde{F}^{\nu \mu}\right)^{2} \\
& \quad+\frac{x \rho_{i} \rho^{i}}{4}\left(\sqrt{x} \mathcal{D}_{\nu}^{\prime \prime} \mathcal{F}^{\nu \mu}+\partial_{\nu} y \tilde{F}^{\nu \mu}-\frac{1}{2} \partial_{\nu} x F^{\nu \mu}\right) \partial^{\rho} x F_{\rho \mu} \\
& \quad+\frac{x \rho_{i} \rho^{i}}{4}\left(\frac{1}{8} \partial_{\rho} x \partial^{\rho} x F^{\nu \mu} F_{\nu \mu}+\frac{1}{4} \partial_{\rho} x \partial^{\rho} y F^{\nu \mu} \tilde{F}_{\nu \mu}-\partial_{\nu} y \partial^{\rho} y F^{\nu \mu} F_{\rho \mu}\right) \\
& \quad+\frac{1}{8}\left\{\mathcal{D}^{\rho} z^{i} \rho_{i j} \bar{f}^{j}\left(F_{\rho \mu}-i \tilde{F}_{\rho \mu}\right)\left[\sqrt{x} \mathcal{D}_{\nu}^{\prime \prime} \mathcal{F}^{\nu \mu}-i \partial_{\nu} y\left(F^{\nu \mu}+i \tilde{F}^{\nu \mu}\right)\right]+\text { h.c. }\right\} \\
& \quad-\frac{1}{32}\left[\mathcal{D}^{\rho} z^{i} \rho_{i j} \bar{f}^{j} \partial_{\rho} x\left(F^{\nu \mu} F_{\nu \mu}-i F^{\nu \mu} \tilde{F}_{\nu \mu}\right)+\text { h.c. }\right] \\
& \quad-\frac{x^{3}}{2} \mathcal{D}^{\rho} z^{i} \mathcal{D}_{\nu} \bar{z}^{\bar{m}} \rho_{i j} \rho_{\bar{m}}^{j} F^{\nu \mu} F_{\rho \mu}+\frac{x^{3}}{8} \mathcal{D}^{\rho} z^{i} \mathcal{D}_{\rho} \bar{z}^{\bar{m}} \rho_{i j} \rho_{\bar{m}}^{j} F^{\nu \mu} F_{\nu \mu}, \\
& 8 L^{\rho} L_{\mu}\left(\bar{M}_{\rho \nu}\right)_{a}^{i}\left(M^{\mu \nu}\right)_{i}^{a}=\frac{x \rho^{i} \rho_{i}}{32}\left(4 \partial_{\nu} y \partial^{\rho} y F_{\rho \mu} F^{\nu \mu}-\partial_{\rho} y \partial^{\rho} y F_{\mu \nu} F^{\mu \nu}\right) \\
& \left\{\left[\hat{L}_{\mu \nu}, \bar{m}\right]_{a}^{i}\left(M^{\mu \nu}\right)_{i}^{a}+\left[\hat{L}_{\mu \nu}, \bar{m}\right]_{\bar{m}}^{a}\left(M^{\mu \nu}\right)_{a}^{\bar{m}}+\text { h.c. }\right\}=\tau_{3}^{x g},
\end{aligned}
$$

and,

$$
\begin{aligned}
& 2\left(\tilde{D}_{\mu} \bar{m}\right)_{a}^{i}\left(\tilde{D}^{\mu} m\right)_{i}^{a}+2\left[L_{\mu}, \bar{m}\right]_{a}^{i}\left[L^{\mu}, m\right]_{i}^{a}=-2 \frac{\partial^{\mu} x}{x^{2}}\left[\left(T_{a} z\right)^{i} K_{i \bar{m}} D_{\mu}\left(T^{a} \bar{z}\right)^{\bar{m}}+\text { h.c. }\right] \\
& \quad+\frac{4}{x} K_{i \bar{m}} D_{\bar{n}}\left(T_{a} \bar{z}\right)^{\bar{m}} \mathcal{D}_{\mu} \bar{z}^{\bar{n}} D_{j}\left(T^{a} z\right)^{i} \mathcal{D}^{\mu} z^{j}+4 x \mathcal{K}_{a}^{b} F_{\mu \nu}^{a} F_{b}^{\mu \nu} \\
& \quad+x \rho_{i} \rho^{i}\left\{K_{i \bar{n}} K_{j \bar{m}} \mathcal{D}^{\mu} z^{j}\left(T_{a} \bar{z}\right)^{\bar{m}}\left[\left(T^{a} z\right)^{i} \mathcal{D}_{\mu} \bar{z}^{\bar{n}}+\left(T^{a} \bar{z}\right)^{\bar{n}} \mathcal{D}_{\mu} z^{i}\right]+\text { h.c. }\right\} \\
& \quad-\partial^{\mu} x \rho_{i} \rho^{i} \mathcal{D}^{a} K_{j \bar{m}}\left[\left(T_{a} z\right)^{j} \mathcal{D}_{\mu} \bar{z}^{\bar{m}}+\left(T_{a} \bar{z}\right)^{\bar{m}} \mathcal{D}_{\mu} z^{j}\right] \\
& \quad-2\left\{K_{j \bar{m}}\left(T_{a} \bar{z}\right)^{\bar{m}} \mathcal{D}_{\mu} z^{j}\left[\rho_{i k}\left(T^{a} z\right)^{i} \mathcal{D}^{\mu} z^{k}+\rho_{\bar{m} \bar{n}}\left(T^{a} \bar{z}\right)^{\bar{m}} \mathcal{D}^{\mu} \bar{z}^{\bar{n}}\right]+\text { h.c. }\right\} \\
& \quad-\frac{1}{2 x} \mathcal{D}_{a}\left\{\rho_{i j} \mathcal{D}_{\mu} z^{i} \bar{f}^{j} K_{k \bar{m}}\left[\left(T^{a} z\right)^{k} \mathcal{D}^{\mu} \bar{z}^{\bar{m}}+\left(T^{a} \bar{z}\right)^{\bar{m}} \mathcal{D}^{\mu} z^{k}\right]+\text { h.c. }\right\} \\
& \quad+2 \mathcal{D}_{a}\left[\rho_{i j} \mathcal{D}_{\mu} z^{i} \mathcal{D}^{\mu} z^{k} \mathcal{D}_{k}\left(T^{a} z\right)^{j}+\text { h.c. }\right]-\frac{2 i \partial_{\mu} y}{x} \mathcal{D}_{a}\left[\rho_{i k}\left(T^{a} z\right)^{i} \mathcal{D}^{\mu} z^{k}-\text { h.c. }\right] \\
& \quad+\left(\partial_{\mu} x \partial^{\mu} x+3 \partial_{\mu} y \partial^{\mu} y\right) \frac{\rho_{i} \rho^{i}}{2} \mathcal{D}+2 x^{2} \mathcal{D}_{i j} \mathcal{D}_{\mu} z^{i} \mathcal{D}^{\mu} \bar{z}^{\bar{m}} \rho_{\bar{m}}^{j} \\
&+\left[\frac{2}{x} F_{a}^{\mu \nu} \partial_{\nu} y K_{i \bar{m}} \mathcal{D}_{\mu} z^{i}\left(T^{a} \bar{z}\right)^{\bar{m}}-x \mathcal{D} \rho^{i} \rho_{i j} \mathcal{D}_{\mu} z^{i}\left(\partial_{\mu} x+2 i \partial_{\mu} y\right)+\text { h.c. }\right] \\
&+4 x F_{\mu \nu}^{a} F_{a}^{\mu \rho} \mathcal{D}_{\rho} z^{i} \mathcal{D}^{\nu} \bar{z}^{\bar{m}} K_{i \bar{m}}+\mathcal{K}_{a}^{a}\left(\frac{\partial_{\mu} x \partial^{\mu} x}{x^{2}}-\frac{\partial_{\mu} y \partial^{\mu} y}{x^{2}}\right) \\
&+2 i\left(\frac{2}{\sqrt{x}} \mathcal{D}_{\mu}^{\prime \prime} \mathcal{F}_{a}^{\mu \nu}-\frac{\partial_{\mu} x}{x} F_{a}^{\mu \nu}\right) K_{i \bar{m}}\left[\mathcal{D}_{\nu} \bar{z}^{\bar{m}}\left(T_{a} z\right)^{i}-\mathcal{D}_{\nu} z^{i}\left(T_{a} \bar{z}\right)^{\bar{m}}\right] .
\end{aligned}
$$


We write the $\chi-\lambda$ contribution to $T$ as

$$
\begin{aligned}
T^{\chi g}= & T_{4}^{\chi g}+T_{3}^{\chi g}+\tau_{3}^{\chi g}+t_{3}^{\chi g}=T_{\chi g}^{\prime}+t_{3}^{\chi g} \\
T_{4}^{\chi g}= & -4\left(\bar{m} M^{\mu \nu}\right)_{a}^{i}\left(\bar{m} M_{\mu \nu}\right)_{i}^{a}+\text { h.c. } \\
= & (x \mathcal{W}+\mathcal{D})\left(x^{2} \rho^{i} \rho_{i} M_{\lambda}^{2}+(a-A) \bar{a}^{i} \frac{f_{i}}{2 x}\right)+\text { h.c. } \\
t_{3}^{\chi g}= & -\frac{16}{3}\left[\left(\tilde{D}^{\sigma} \bar{M}_{\sigma \mu}\right)_{a}^{i}\left(\tilde{D}_{\rho} M^{\rho \mu}\right)_{i}^{a}+L^{\sigma} L_{\rho}\left(\bar{M}_{\sigma \mu}\right)_{a}^{i}\left(M^{\rho \mu}\right)_{i}^{a}\right] \\
& +\frac{16 i}{3} L^{\sigma}\left[\left(\bar{M}_{\sigma \mu}\right)_{a}^{i}\left(\tilde{D}_{\rho} M^{\rho \mu}\right)_{i}^{a}-\text { h.c. }\right] \\
\tau_{3}^{\chi g}= & -\frac{\rho^{i} \rho_{i}}{2} \partial_{\mu} y \partial_{\nu} x F_{a}^{\mu \nu} \mathcal{D}^{a}+\frac{\partial_{\mu} y}{2 x} F_{\rho \nu}^{a}\left(\partial^{\rho} x \tilde{F}_{a}^{\mu \nu}-\partial^{\rho} y F_{a}^{\mu \nu}\right) \\
T_{3}^{\chi g}= & 2 i L^{\rho} m_{i}^{a} \tilde{D}_{\rho} \bar{m}_{a}^{i}+\text { h.c. } \\
= & -2 \partial_{\mu} y \partial^{\mu} y \rho_{i} \rho^{i} \mathcal{D}+2 \frac{\partial_{\mu} y}{x} F_{a}^{\mu \nu}\left[K_{i \bar{m}} \mathcal{D}_{\nu} z^{i}\left(T^{a} \bar{z}\right)^{\tilde{m}}+\text { h.c. }\right]+\frac{\partial_{\mu} x \partial_{\nu} y}{x^{2}} F_{a}^{\mu \nu} \mathcal{D}^{a} \\
& -\frac{i \partial_{\mu} y}{2 x}\left\{\mathcal{D}^{\mu} z^{i}\left[\mathcal{D} \bar{f}^{j} \rho_{i j}+\frac{4}{x} K_{j \bar{m}}\left(T^{a} \bar{z}\right)^{\tilde{m}} D_{i}\left(T^{a} z\right)^{j}\right]-\text { h.c. }\right\} \\
& +\frac{2 i}{x} \mathcal{D}^{a} \partial^{\mu} y\left[\rho_{i j}\left(T_{a} z\right)^{i} \mathcal{D}_{\mu} z^{j}-\text { h.c. }\right]
\end{aligned}
$$

where

$$
\begin{gathered}
8 i L^{\sigma}\left(\bar{M}_{\sigma \mu}\right)_{a}^{i}\left(\tilde{D}_{\rho} M^{\rho \mu}\right)_{i}^{a}+\text { h.c. }=\frac{i}{32}\left[\mathcal{D}^{\rho} z^{i} \rho_{i j} \bar{f}^{j}\left(4 \partial_{\nu} y F_{\mu \rho}^{a} F_{a}^{\mu \nu}-\partial_{\rho} y F_{a}^{\mu \nu} F_{\mu \nu}^{a}\right)-\text { h.c. }\right] \\
+\frac{x \rho_{i} \rho^{i}}{4} \partial^{\rho} y\left[\tilde{F}_{\rho \nu}^{a}\left(\sqrt{x} \mathcal{D}_{\mu}^{\prime \prime} \mathcal{F}_{a}^{\mu \nu}+\partial_{\mu} y \tilde{F}_{a}^{\mu \nu}-\partial_{\mu} x F_{a}^{\mu \nu}\right)+\partial_{\nu} y F_{\mu \rho}^{a} F_{a}^{\mu \nu}\right] .
\end{gathered}
$$

In addition we have

$$
\begin{gathered}
\operatorname{Tr}\left(\hat{G}_{\Phi}^{x g}\right)^{2}=4\left(G_{\mu \nu}^{g z}\right)_{a \rho, i}\left(G_{\mu \nu}^{g z}\right)^{i, a \rho}=\operatorname{Tr}\left(\hat{G}_{\Theta}^{\chi g}\right)^{2} \\
=64\left(\tilde{D}_{\mu} \bar{M}_{\nu \rho}\right)_{a}^{i}\left(\tilde{D}^{\mu} M^{\nu \rho}-\tilde{D}^{\nu} M^{\mu \rho}\right)_{i}^{a} \\
-128 i\left[L^{\nu}\left(\bar{M}_{\nu \rho}\right)_{a}^{i}\left(\tilde{D}_{\mu} M^{\mu \rho}\right)_{i}^{a}-\text { h.c. }\right], \\
64\left(\tilde{D}_{\mu} \bar{M}_{\nu \rho}\right)_{a}^{i}\left(\tilde{D}^{\mu} M^{\nu \rho}-\tilde{D}^{\nu} M^{\mu \rho}\right)_{i}^{a}=-2 x^{2} \rho_{i} \rho^{i}\left(\mathcal{D}_{\mu}^{\prime \prime} \mathcal{F}_{a}^{\mu \nu}+\frac{\partial_{\mu} y}{\sqrt{x}} \tilde{F}_{a}^{\mu \nu}\right)^{2} \\
-\frac{1}{4}\left\{\mathcal{D}^{\rho} z^{i} \rho_{i j} \bar{f}^{j}\left[F_{a}^{\mu \nu} F_{\mu \nu}^{a} \partial_{\rho} x-i \tilde{F}_{a}^{\mu \nu} F_{\mu \nu}^{a}\left(\partial_{\rho} x+i \partial_{\rho} y\right)+4 i \partial_{\nu} y F_{\mu \rho}^{a} F_{a}^{\mu \nu}\right]+\text { h.c. }\right\} \\
+\left(\sqrt{x} \mathcal{D}_{\mu}^{\prime \prime} \mathcal{F}_{a}^{\mu \nu}+\partial_{\mu} y \tilde{F}_{a}^{\mu \nu}\right)\left\{2 x \rho^{i} \rho_{i} \partial^{\rho} x F_{\rho \nu}^{a}+\left[\left(F_{\rho \nu}^{a}-i \tilde{F}_{\rho \nu}^{a}\right) \mathcal{D}^{\rho} z^{i} \rho_{i j} \bar{f}^{j}+\text { h.c. }\right]\right\} \\
+F_{a}^{\mu \nu} F_{\mu \nu}^{a}\left[\frac{x}{4} \rho_{i} \rho^{i} \partial_{\rho} x \partial^{\rho} x+x^{3} \rho_{i j} \rho_{\bar{m}}^{j} \mathcal{D}_{\rho} z^{i} \mathcal{D}^{\rho} \bar{z}^{\tilde{m}}\right]+\frac{x \rho_{i} \rho^{i}}{2} \tilde{F}_{a}^{\mu \nu} F_{\mu \nu}^{a} \partial^{\rho} y \partial_{\rho} x \\
-F_{a}^{\mu \rho} F_{\nu \rho}^{a}\left[x \rho_{i} \rho^{i}\left(\partial_{\mu} x \partial^{\nu} x+2 \partial_{\mu} y \partial^{\nu} y\right)+4 x^{3} \rho_{i j} \rho_{m}^{j} \mathcal{D}_{\mu} z^{i} \mathcal{D}^{\nu} \bar{z}^{m}\right],
\end{gathered}
$$


Using the classical equations of motion (A.17-20), we obtain, with $k^{1}=-4 \mathcal{K}_{a}^{a}$,

$$
\begin{aligned}
& \frac{1}{2} \operatorname{STr} H_{\chi g}^{2}=-T_{\chi g}^{\prime}+\left(\mathcal{D}_{\mu} z^{i} \mathcal{D}^{\mu} \bar{z}^{\bar{m}}+\bar{A}^{i} A^{\bar{m}} e^{-K}\right)\left(k_{i \bar{m}}^{1}-\frac{4}{x}\left(T^{a} z\right)^{j}\left(T_{a} \bar{z}\right)^{\bar{n}} R_{i \bar{m} j \bar{n}}\right) \\
& -e^{-K}\left(k_{i}^{1} \bar{A}^{i} A+\text { h.c. }\right)-\frac{4 x^{2} \rho^{i} \rho_{i}}{\sqrt{g}} \Delta_{\mathcal{D}} \mathcal{L}-\frac{x^{2} \rho^{i} \rho_{i}}{g x} \mathcal{L}_{a \mu} \mathcal{L}^{a \mu} \\
& +\frac{2 x \rho^{i} \rho_{i}}{\sqrt{g}}\left[i \mathcal{L}^{\alpha \mu}\left(K_{i \bar{m}} \mathcal{D}_{\mu} \bar{z}^{\bar{m}}\left(T_{a} z\right)^{i}-\text { h.c. }\right)+\mathcal{D}^{a}\left(T_{a} z\right)^{I} \mathcal{L}_{I}\right] \\
& +\frac{1}{\sqrt{g}} \mathcal{L}_{a}^{\rho}\left\{x \rho^{i} \rho_{i} \partial^{\mu} y \tilde{F}_{\mu \rho}^{a}+\left[\frac{\bar{f}^{i}}{2} \rho_{i j} \mathcal{D}^{\nu} z^{j}\left(F_{\nu \rho}^{a}-i \tilde{F}_{\nu \rho}^{a}\right)+\text { h.c. }\right]\right\} \\
& -\frac{1}{12} \operatorname{STr} \hat{G}_{\chi g}^{2}-t_{3}^{\chi g}+4 x^{2} \rho^{i} \rho_{i} \mathcal{D}\left[3 M_{\psi}^{2}+\hat{V}-e^{-K} a \bar{a}\right]+10 x^{2} \rho^{i} \rho_{i} \mathcal{D} M_{\lambda}^{2} \\
& +4 x\left[\rho_{i j}\left(T^{a} z\right)^{i}\left(T^{b} z\right)^{j}\left(\mathcal{W}_{a b}+\frac{1}{2 x^{2}} \mathcal{D}_{a} \mathcal{D}_{b}\right)+\text { h.c. }\right]-4 M_{\psi}^{2} \mathcal{K}_{a}^{a} \\
& +2\left[i x \rho_{\bar{m} i j} \mathcal{D}_{\nu} \bar{z}^{\bar{m}}\left(T^{a} z\right)^{i} \mathcal{D}_{\mu} z^{j}\left(F_{a}^{\mu \nu}-i \tilde{F}_{a}^{\mu \nu}\right)+\text { h.c. }\right] \\
& -\frac{3 x \rho_{i} \rho^{i}}{4}\left(F^{\nu \mu}-i \tilde{F}^{\nu \mu}\right)\left(F_{\rho \mu}+i \tilde{F}_{\rho \mu}\right)\left(\partial_{\nu} x \partial^{\rho} x+\partial_{\nu} y \partial^{\rho} y\right) \\
& -\left(\frac{1}{x^{2}}+\rho^{i} \rho_{i}\right) \partial_{\mu} y \partial_{\nu} x \mathcal{D}^{a} F_{a}^{\mu \nu}+\frac{\rho_{i} \rho^{i}}{2} \mathcal{D}\left(5 \partial_{\mu} x \partial^{\mu} x+3 \partial_{\mu} y \partial^{\mu} y\right) \\
& -i x \rho^{i} \rho_{i} K_{i \bar{m}}\left[\mathcal{D}^{\rho} \bar{z}^{\bar{m}}\left(T_{a} z\right)^{i}-\mathcal{D}^{\rho} z^{i}\left(T_{a} \bar{z}\right)^{\bar{m}}\right] \partial^{\mu} y \tilde{F}_{\mu \rho}^{a} \\
& +\left\{\mathcal{W}\left[2 x^{3} \rho^{i} \rho_{i} M_{\lambda}^{2}+(a-A) f_{i} \bar{a}^{i} e^{-K}-\partial_{\rho} f \mathcal{D}^{\rho} z^{j} \rho_{i j} \frac{\bar{f}^{i}}{2}\right]+\text { h.c. }\right\} \\
& -2 x^{2}\left[\left(F_{a}^{\nu \mu} F_{\rho \mu}^{a}-i F_{a}^{\nu \mu} \tilde{F}_{\rho \mu}^{a}\right) \mathcal{D}^{\rho} z^{i} \mathcal{D}^{\nu} z^{j} \rho_{i j}+\text { h.c. }\right] \\
& +2 \frac{\partial^{\mu} x}{\sqrt{x}} F_{\mu \rho}^{a} \mathcal{D}_{\nu}^{\prime \prime} \mathcal{F}_{a}^{\nu \rho}+\frac{\partial_{\nu} y \partial^{\nu} x}{x} F_{a}^{\mu \rho} \tilde{F}_{\mu \rho}^{a}+F_{a}^{\nu \mu} F_{\rho \mu}^{a}\left(\frac{\partial_{\nu} x \partial^{\rho} x}{x}-\frac{\partial_{\nu} y \partial^{\rho} y}{x}\right) \\
& -e^{-K} \mathcal{D}\left(2 a_{i} \bar{a}^{i}+16 a \bar{a}\right)+2 \mathcal{D}\left(3 \hat{V}+17 M_{\psi}^{2}\right)+4 M_{\lambda}^{2}\left(\mathcal{D}+\mathcal{K}_{a}^{a}\right) \\
& +\frac{e^{-K}}{x}\left\{2 \mathcal{D}^{a}\left(T_{a} z\right)^{i} A_{i j}\left(\bar{a}^{j}-2 \bar{A}^{j}\right)-a_{i}(\bar{a}-\bar{A})\left[\bar{f}^{i} \mathcal{D}-2\left(T^{a} z\right)^{i} \mathcal{D}_{a}\right]+\text { h.c. }\right\} \\
& -4 x \mathcal{K}_{a}^{b} F_{\mu \nu}^{a} F_{b}^{\mu \nu}-\rho_{i} \rho^{i}\left[\left(\partial^{\mu} x+2 i \partial^{\mu} y\right) K_{j \bar{m}}\left(T_{a} z\right)^{j} \mathcal{D}_{\mu} \bar{z}^{\bar{m}}+\text { h.c. }\right] \mathcal{D}^{a} \\
& -\frac{i}{2} K_{i \bar{m}}\left(\mathcal{D}^{\nu} \bar{z}^{\bar{m}}\left(T_{a} z\right)^{i}-\mathcal{D}^{\nu} z^{i}\left(T_{a} \bar{z}\right)^{\bar{m}}\right)\left[\bar{f}^{i} \rho_{i j} \mathcal{D}^{\rho} z^{j}\left(F_{\rho \nu}^{a}-i \tilde{F}_{\rho \nu}^{a}\right)+\text { h.c. }\right] \\
& -2\left\{K_{j \bar{m}}\left(T_{a} \bar{z}\right)^{\bar{m}} \mathcal{D}_{\mu} z^{j}\left[\rho_{i k}\left(T^{a} z\right)^{i} \mathcal{D}^{\mu} z^{k}+\rho_{\bar{\ell} \bar{n}}\left(T^{a} \bar{z}\right)^{\bar{\ell}} \mathcal{D}^{\mu} \bar{z}^{\bar{n}}\right]+\text { h.c. }\right\} \\
& +\frac{1}{2 x} \mathcal{D}_{a}\left\{\rho_{i j} \mathcal{D}_{\mu} z^{i} \bar{f}^{j} K_{k \bar{m}}\left[\left(T^{a} z\right)^{k} \mathcal{D}^{\mu} \bar{z}^{\bar{m}}+\left(T^{a} \bar{z}\right)^{\bar{m}} \mathcal{D}^{\mu} z^{k}\right]+\text { h.c. }\right\} \\
& +\left\{\rho_{i j} \mathcal{D}^{\nu} z^{i}\left[2 \mathcal{D}_{a} \mathcal{D}_{k}\left(T^{a} z\right)^{j} \mathcal{D}_{\nu} z^{k}+\vec{f}^{j} F_{a}^{\mu \rho}\left(\frac{\partial_{\mu} x}{2} F_{\nu \rho}^{a}-\frac{\partial_{\nu} x}{8} F_{\mu \rho}^{a}\right)\right]+\text { h.c. }\right\} \\
& -4 x F_{\mu \nu}^{a} F_{a}^{\mu \rho} \mathcal{D}_{\rho} z^{i} \mathcal{D}^{\nu} \bar{z}^{\bar{m}} K_{i \bar{m}}+\mathcal{K}_{a}^{a}\left(\frac{\partial_{\mu} x \partial^{\mu} x}{x^{2}}+\frac{\partial_{\mu} y \partial^{\mu} y}{x^{2}}\right) \\
& -2 i\left(2 \frac{\mathcal{D}_{\mu}^{\prime \prime} \mathcal{F}_{a}^{\mu \nu}}{\sqrt{x}}-\frac{\partial_{\mu} x}{x} F_{a}^{\mu \nu}\right) K_{i \bar{m}}\left[\mathcal{D}_{\nu} \bar{z}^{\bar{m}}\left(T_{a} z\right)^{i}-\mathcal{D}_{\nu} z^{i}\left(T_{a} \bar{z}\right)^{\bar{m}}\right]
\end{aligned}
$$




$$
\begin{aligned}
& +\frac{x \rho_{i} \rho^{i}}{4}\left(F_{a}^{\mu \nu} F_{\mu \nu}^{a} \partial_{\rho} x \partial^{\rho} x+F_{a}^{\mu \nu} \tilde{F}_{\mu \nu}^{a} \partial_{\rho} x \partial^{\rho} y\right) \\
& -\frac{13 x \rho_{i} \rho^{i}}{96}\left(F_{a}^{\mu \nu} F_{\mu \nu}^{a} \partial_{\rho} y \partial^{\rho} y-4 F_{a}^{\mu \rho} F_{\nu \rho}^{a} \partial_{\mu} y \partial^{\nu} y\right) \\
& +2 x^{2} \rho_{i j} \rho_{\bar{m}}^{j} \mathcal{D} \mathcal{D}_{\rho} z^{i} \mathcal{D}^{\rho} \bar{z}^{\bar{m}}
\end{aligned}
$$

$$
\begin{aligned}
\frac{1}{12} \mathrm{STr} \hat{G}_{\chi g}^{2}= & -t_{3}^{\chi g}-\frac{x^{2} \rho_{i} \rho^{i}}{4}\left(\mathcal{D}_{\mu}^{\prime \prime} \mathcal{F}_{a}^{\mu \nu}+\frac{\partial_{\mu} y}{\sqrt{x}} \tilde{F}_{a}^{\mu \nu}\right)^{2} \\
& +x \rho_{i} \rho^{i}\left[\frac{\partial^{\rho} x}{4} F_{\rho \nu}^{a}\left(\sqrt{x} \mathcal{D}_{\mu}^{\prime \prime} \mathcal{F}_{a}^{\mu \nu}+\partial_{\mu} y \tilde{F}_{a}^{\mu \nu}\right)+\frac{\partial^{\rho} y \partial_{\rho} x}{16} \tilde{F}_{a}^{\mu \nu} F_{\mu \nu}^{a}\right] \\
& +\frac{1}{8}\left[\left(F_{\rho \nu}^{a}-i \tilde{F}_{\rho \nu}^{a}\right) \mathcal{D}^{\rho} z^{i} \rho_{i j} \bar{f}^{j}+\text { h.c. }\right]\left(\sqrt{x} \mathcal{D}_{\mu}^{\prime \prime} \mathcal{F}_{a}^{\mu \nu}+\partial_{\mu} y \tilde{F}_{a}^{\mu \nu}\right) \\
& +\frac{1}{32} F_{a}^{\mu \nu} \tilde{F}_{\mu \nu}^{a}\left[i\left(\partial_{\rho} x+i \partial_{\rho} y\right) \mathcal{D}^{\rho} z^{i} \rho_{i j} \bar{f}^{j}+\text { h.c. }\right] \\
& -\frac{x^{3}}{2} \rho_{i j} \rho_{\tilde{m}}^{j}\left(F_{a}^{\mu \rho} F_{\nu \rho}^{a} \mathcal{D}_{\mu} z^{i} \mathcal{D}^{\nu} \bar{z}^{m}-\frac{1}{4} F_{a}^{\mu \nu} F_{\mu \nu}^{a} \mathcal{D}_{\rho} z^{i} \mathcal{D}^{\rho} \bar{z}^{m}\right) \\
& +\frac{x \rho_{i} \rho^{i}}{32}\left[F_{a}^{\mu \nu} F_{\mu \nu}^{a} \partial_{\rho} x \partial^{\rho} x-4 F_{a}^{\mu \rho} F_{\nu \rho}^{a}\left(\partial_{\mu} x \partial^{\nu} x+2 \partial_{\mu} y \partial^{\nu} y\right)\right] \\
& -\frac{1}{32}\left[\left(F_{\mu \nu}^{a} \partial_{\rho} x+4 i F_{\mu \rho}^{a} \partial_{\nu} y\right) F_{a}^{\mu \nu} \rho_{i j} \bar{f}^{i} \mathcal{D}^{\rho} z^{j}+\text { h.c. }\right] \\
& +\frac{x \rho_{i} \rho^{i}}{48}\left(F_{a}^{\mu \nu} F_{\mu \nu}^{a} \partial_{\rho} y \partial^{\rho} y-4 F_{a}^{\mu \rho} F_{\nu \rho}^{a} \partial_{\mu} y \partial^{\nu} y\right) .
\end{aligned}
$$




\section{Bibliography}

[1] H.P. Nilles, Phys Rep. 110, (1984).

[2] H. Haber, in Recent Directions on Particle Theory, J. Harvey and J. Polchinski Eds. (TASI, 1992).

[3] J. Bagger, "Weak-scale Supersymmetry: Theory and Practice", hep-ph/9604232; M. Dine, "Supersymmetry Phenomenology - With a Broad Brush", in Strings, Fields and Duality, TASI-96, hep-ph/9612389.

[4] U.Amaldi, W. de Boer, H. Furstenau, Phys. Lett. 260B: 447, (1991); P. Langacker, M. Luo Phys. Rev. D44, 817 (1991).

[5] N. Seiberg, E. Witten, Nucl. Phys. B426 19, (1994).

[6] K. Intrilligator, N. Seiberg, "Lectures on Supersymmetric Gauge Theories and Electric-Magnetic Duality", hep-th/9509066.

[7] B. Zumino, Phys. Lett. 87B: 203, (1979).

[8] R. Wald, "General Relativity", (1984) University of Chicago Press, Chicago, IL.

[9] M. Nakahara, "Geometry, Topology and Physics" (1990), Adam Hilgar, Bristol.

[10] J. Wess and J. Bagger, "Supersymmetry and Supergravity"," , Second Edition, (1992), Princeton Uiversity Press, Princeton, NJ.

[11] E. Cremmer, B. Julia, J.Scherk, S. Ferrara, L. Girardello, and P. Van Nieuwenhuizen, Phys. Lett. B79: 231 (1978); and Nucl. phys. B147: 105, (1979)

[12] P. Binétruy, G. Girardi and R. Grimm, Annecy preprint LAPP-TH-275-90, (1990).

[13] P. Binétruy, G. Girardi, R. Grimm and M. Muller, Phys. Lett. 189B: 83 (1987).

[14] M. Green, J. Schwarz, E. Witten, "Superstring Theory", vols. 1 and 2, CUP, Cambridge (1987)

[15] F.Gliozzi, J. Scherk, D. Olive, Phys. Lett., B65, 282 (1976); and Nucl. Phys. B122, 253 (1977). 
[16] J. Schwarz, "Lectures on String Dualities and M Theory", TASI , (1996).

[17] D. Gross, J. Harvey, E. Martinec, R. Rhom, Nucl. Phys. B256, 253 (1985); Nucl. Phys. B267, 75 (1986).

[18] D. Bailin and A. Love, "Supersymmetric Gauge Field Theory and String Theory", (1994) IOP Publishing, Bristol.

[19] P. Binétruy, "Compactification and Phenomenology of Superstrings" Preprint LAPP-TH-218-88, (1988).

[20] M. Green, J. Schwarz, Phys. Lett. B149, 117 (1984).

[21] K.S. Narain, Phys. Lett. B169 41 (1986); K.S. Narian, M.H. Sarmadi, E. Witten, Nucl. Phys. B279, 369 (1987).

[22] P. Candelas, G. Horowitz, A. Strominger, E. Witten, Nucl. Phys. B : 258 , (1985).

[23] L. Dixon, J. Harvey, C. Vafa, E. Witten, Nucl. Phys. B261: 678 (1985); and Nucl. Phys. B274: 283 (1985).

[24] L. I. Ibáñez, H.P. Nilles, F. Quevedo, Phys. Lett. B187, 25 (1987); L. I. Ibáñez, J. Kim, H.P. Nilles, F. Quevedo, Phys. Lett. B191, 3 (1987); D. Bailin, A. Love, S.Thomas, Phys. Lett. B188, 198 (1987).

[25] L. Dixon, V. Kaplunovsky and J. Louis, Nucl. Phys. B355: 649 (1991).

[26] G.L. Cardoso and B.A. Ovrut, Nucl. Phys. B369: 351 (1992); J.-P. Derendinger, S. Ferrara, C. Kounnas and F. Zwirner, Phys. Lett. B271: 307 (1991); P. Binétruy, G. Girardi, R. Grimm and M. Müller, Phys. Lett. $B 265111$ (1991); P. Adamietz, P. Binétruy, G. Girardi and R. Grimm, Nucl. Phys. B401: 257 (1993); P. Mayr and S. Stieberger, Nucl. Phys. B412: 502 (1994); M.K. Gaillard and T.R. Taylor, Nucl. Phys. B381: 577 (1992); V. Kaplunovsky and J. Louis, Nucl. Phys. B422: 57 (1994).

[27] P. Binétruy, M.K. Gaillard and T.R. Taylor, Nucl. Phys. B455: 97 (1995); P. Binétruy and M.K. Gaillard, Phys. Lett. B365: 87 (1996).

[28] I. Antoniadis, E. Gava, K.S. Narain and T.R. Taylor, Nucl. Phys. 428: 282 (1994). 
[29] M.K. Gaillard and T.R. Taylor, Nucl. Phys. B381: 577 (1992).

[30] M.K. Gaillard and V. Jain, Phys. Rev. D49: 1951 (1994).

[31] M.K. Gaillard, V. Jain and K. Saririan, Phys. Rev. D55: 883 (1997).

[32] M.K. Gaillard, V. Jain and K. Saririan, Phys. Lett. B 387: 520 (1996).

[33] R. Barbieri, S. Ferrara, L. Maiani, F. Palumbo and C.A. Savoy, Phys. Lett. 115B: 212 (1982).

[34] I. Antoniadis, E. Gava, K.S. Narain and T.R. Taylor, Nucl. Phys. B407: 706 (1993).

[35] G.K. 't Hooft and M. Veltman, Ann. Inst. Henri Poincaré 20: 69 (1974).

[36] R. Barbieri and C. Cecotti, Z. Phys. C17: 183 (1983); P. Binétruy and M.K. Gaillard, Nucl. Phys. B254: 388 (1985).

[37] J. W. Burton, M.K. Gaillard and V. Jain, Phys. Rev. D41: 3118 (1990).

[38] M. Srednicki and S. Theisen, Phys. Rev. Lett. 54: 278 (1985).

[39] C. Chiou-Lahanas, A. Kapella-Economu, A.B. Lahanas and X.N. Maintas, Phys. Rev. D42: 469 (1990) and Phys. Rev. D45: 534 (1992).

[40] P. Binétruy and M.K. Gaillard, Nucl. Phys. B312: 341 (1989).

[41] M.K. Gaillard and V. Jain, Phys. Rev. D46: 1786 (1992).

[42] E. Cremmer, S. Ferrara, L. Girardello, and A. Van Proeyen, Nucl. Phys. B212: 413 (1983).

[43] P. Ginsparg, Phys. Lett. B197: 139 (1987).

[44] P. Binétruy and M.K. Gaillard, Phys. Lett. 232B: 83 (1989) and Nucl. Phys. B358: 121 (1991).

[45] M.K. Gaillard, Phys.Lett. B342: 125 (1995) and Phys. Lett B347: 284 (1995).

[46] G.A. Vilkoviski, Nucl. Phys. B234: 125 (1984); E.S. Fradkin and A.A. Tseytlin, Nucl. Phys. B234: 509 (1984). 
[47] S. Honerkamp, Nucl. Phys. B36: 130 (1973); L. Alvarez-Gaumé, D. Freedman, S. Mukhi, Ann. Phys. (N.Y.) 134: 85, (1981); S. Mukhi, Nucl. Phys. B264: 640 (1986).

[48] M.K. Gaillard, Nucl. Phys. B268: 669 (1986).

[49] P. Binétruy and M.K. Gaillard, Phys. Lett. 220B: 68 (1989).

[50] V. Jain,Phys. Rev. D40:1213 (1989).

[51] O. Cheyette and M.K. Gaillard, Phys. Lett. 197B: 205 (1987).

[52] M.K. Gaillard and S.-J. Rey, in preparation.

[53] L. Rosenberg, Phys. Rev. 129: 2786 (1963); S.L. Adler, Phys. Rev. 177: 2060 (1969).

[54] P. van Nieuwenhuizen, Phys. Rep. 68: 189 (1981).

[55] S.W. Christensen and M.J. Duff, Nucl. Phys. B154: 301 (1979).

[56] I. Antoniadis, E. Gava, K.S. Narain and T.R. Taylor, Nucl. Phys. B413: 162 (1994); these terms appear only at two loops (T.R. Taylor, private communication); however there could be other terms with no bosonic components that arise at one loop.

[57] P. Binétruy, S. Dawson, M.K. Gaillard and I. Hinchliffe, Phys. Rev. D37: 2633 (1988).

[58] M. A. Shifman and A. I. Vainshtein, Nucl. Phys. B277: 456 (1986); and Nucl. Phys. B359: 571 (1991).

[59] W. Seigel, Phys. Lett. 211B: 55 (1988).

[60] E.S. Fradkin and A.A. Tseytlin, Ann. Phys. 162: 31 (1985). This paper showed equivalence up to finite topological anomalies; subsequently full equivalence has been shown; S.J. Rey, private communication.

[61] J.-P. Derendinger, F. Quevedo, M. Quiros, Nucl. Phys. B428: 282 (1994).

[62] S. Ferrara and B. Zumino, Nucl. Phys. 79: 413 (1974). 
[63] H.P. Nilles, Phys. Lett. 115B: 455 (1982).

[64] P. Binétruy, M.K. Gaillard and T.R. Taylor, Nucl. Phys. B455: 97 (1995).

[65] I. Antoniadis, E. Gava, K.S. Narain and T.R. Taylor, Nucl. Phys. B432: 187 (1994).

[66] M. K. Gaillard and B. Zumino, Nucl. Phys. B193: 221 (1981); S. Ferrara, J. Scherk., and B. Zumino, Nucl. Phys. 121: 393 (1977).

[67] P. Binétruy and M.K. Gaillard, Phys. Lett. B365: 87 (1996).

[68] K. Saririan, Phys. Rev. D55: 4839 (1997).

[69] M. K. Gaillard, and R. Xiu, Phys. Lett. B296: 71 (1992) ; R. Xiu, Phys. Rev. D49: 6656 (1994) ; S. P. Martin and P. Ramond, Phys. Rev. D51: 6515 (1995); K.R. Dienes and A.E. Farraggi, Phys. Rev. Lett. 75: 2646 (1995).

[70] G. Veneziano and S. Yankielowicz, Phys. Lett. B113: 231 (1982).

[71] E. Witten, Nucl. Phys. B202: 253 (1982).

[72] H. P. Nilles, Phys. Lett. B115: 193 (1982); S. Ferrara, L. Girardello, H. P. Nilles, Phys. Lett. B125 457 (1983).

[73] P. Binétruy and M.K. Gaillard, Phys. Lett. B253: 119 (1991).

[74] M. Dine, R. Rhom, N. Seiberg, and E. Witten, Phys. Lett. B156: 55 (1985).

[75] G.L. Cardoso and B.A. Ovrut, Nucl. Phys. B369: 351 (1992); ibid., Nucl. Phys. B392: 315 (1993); ibid.,Nucl. Phys. B418: 535 (1994); S. Ferrara abd M. Villasante, Phys. Lett. B186: 87 (1987); P. Binétruy, G. Girardi, R. Grimm and M. Müller, Phys. Lett. B265 111 (1991); P. Adamietz, P. Binétruy, G. Girardi and R. Grimm, Nucl. Phys. B401: 257 (1993); J.P. Derendinger, F. Quevedo, M. Quirós, Nucl. Phys. B428: 228 (1994).

[76] I. Antoniadis, K.S. Narain, and T.R. Taylor, Phys. Lett. B267: 37 (1991).

[77] M.K. Gaillard and T.R. Taylor, Nucl. Phys. B381: 577 (1992).

[78] A. Font, L. I. Ibáñez, D. Lüst, F. Quevedo, Phys. Lett. 241: 35 (1990). 
[79] A. Sen, Int. J. Mod. Phys. A9: 3707 (1994), J. H. Schwarz and A. Sen, Phys. Lett. B312: 105 (1993); Phys. Lett. B411: 105 (1994); I. Girardello, A. Giveon, M. Porratti, A. Zaffaroni, Nucl. Phys. B448 127 (1995).

[80] Z. Lalak, A. Niemeyer, H. P. Nilles, Nucl. Phys.B453: 100, 1995.

[81] B. de Carlos, J. Casas, and C. Muñoz, Phys. Lett. B263: 248 (1991); D. Lüst and C. Muñoz, CERN-Th 6358/91 (1991).

[82] S. Chaudhuri, G. Hockney, and J. Lykken, Nucl. Phys.B469: 357 (1996).

[83] V. Kaplunovsky and J. Louis, Nucl. Phys. B422: 57 (1994).

[84] V. Kaplunovsky and J. Louis, Nucl. Phys. B444: 191 (1995).

[85] K. Saririan, in Progress.

[86] T. Banks and M. Dine, Phys. Rev. D:50 7454 (1994).

[87] P. Binétruy, M. K. Gaillard and Y-Y. Wu, Nucl. Phys. B481:, 109 (1996). 